\title{
NEW MODELS FOR TIME-CUMULATIVE BEHAVIOR OF \\ UNCONVENTIONAL RESERVOIRS --- DIAGNOSTIC RELATIONS, PRODUCTION FORECASTING, AND EUR METHODS
}

\author{
A Thesis \\ by \\ WAJID YOUSUF
}

Submitted to the Office of Graduate Studies of Texas A\&M University

in partial fulfillment of the requirements for the degree of

MASTER OF SCIENCE

Chair of Committee,

Committee Members,

Head of Department,
Thomas A. Blasingame

Peter P. Valkó

Eduardo Gildin

A. Daniel Hill

December 2017

Major Subject: Petroleum Engineering 


\begin{abstract}
This work presents the development of two new rate-time relations which are based on self-growth limiting time-cumulative relations. This self-limiting feature provides an inherent upper limit on ultimate reserves and eliminates the need for a terminal decline component as is required in other time-rate relations. Another inherent advantage of this approach is that these new models introduce EUR as a regression parameter instead of using the "intercept rate" as the general regression parameter (as is the case in the Arps' time-rate relations and most subsequent models).

As validation of these two new relations we employ synthetic solutions (i.e., reservoir simulation) as well as field performance data taken from a well-documented tight gas case and from two North American shale gas cases. As a summary statement, the new relations tend to be more "conservative" estimators (like the power-law exponential and other statistical relations (e.g., the Logistic Growth Model)) and less like the Arps' hyperbolic family of relations. In general, the new models match all of the cases reasonably well, but (as noted), the forecasted production and estimated ultimate recovery (EUR) extrapolations tend to be conservative. Unfortunately, the new models do not provide any direct diagnostic characteristics where the parameters in these relations could be estimated directly (e.g., as in the case of using the slope and/or intercept of a straight-line trend).
\end{abstract}

Moreover, in this work we do provide a series of "time-cumulative" plotting functions in an attempt to provide data diagnostics which are less affected by data noise inherent in production data. These relations appear to be potentially useful - however; a concern remains regarding the introduction of new data diagnostic functions as the "Arps'" functions $(D(t)$ and $b(t))$ are the standard variables used in practice and it is unlikely that industry practice will embrace new functions which do not provide significant advantages over the Arps' relations. Furthermore, we show that definitions of these diagnostics help us formulate some special plotting relations for proposed Modified Wiorkowski Model, which can be used to determine regression parameters directly from historical production data, reducing uncertainty.

Finally, we present a study to integrate time-rate model parameters with fundamental completion reservoir properties (i.e., fracture conductivity $\left(F_{c}\right)$, fracture half length (xf), formation permeability (k) and 30 year EUR $\left.\left(E U R_{30 \mathrm{yr}}\right)\right)$ using parametric correlations. Previously, work by Ilk and Askabe has shown that it is possible to correlate reservoir/well properties that are estimated using model-based production data analysis with model parameters of time-rate relations. We demonstrate the application of a methodology that allows formulating multivariate parametric correlations to integrate completion/reservoir parameters with time-rate model parameters. 


\section{DEDICATION}

I dedicate this work to my family. 


\section{ACKNOWLEDGEMENTS}

I am really thankful to Dr. Tom Blasingame, my committee chair, for his continuous support, help and encouragement. This thesis would not have been possible without his patience and understanding. I would also like to thank Dr. Peter Valko and Dr. Eduardo Gildin for serving as members of my committee.

I am also thankful to IIE and Fulbright program for providing me with this educational opportunity, as well as funding and supervision during my studies.

Finally, I am thankful to my family for their love and support. 


\section{CONTRIBUTORS AND FUNDING SOURCES}

\section{Contributors}

This work was supported by a thesis committee consisting of Professor Tom Blasingame, Professor Peter Valko and Professor Eduardo Gildin of the Department of Petroleum Engineering.

\section{Funding Sources}

Graduate study was supported by a Fulbright Masters Scholarship award and a graduate fellowship from Texas A\&M University. 


\section{TABLE OF CONTENTS}

Page

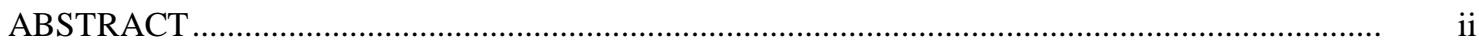

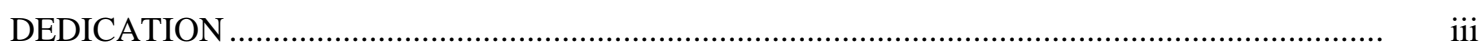

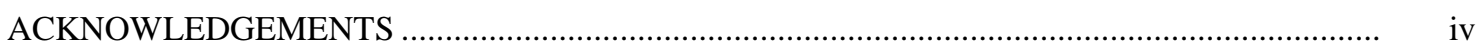

CONTRIBUTORS AND FUNDING SOURCES ….................................................................. v

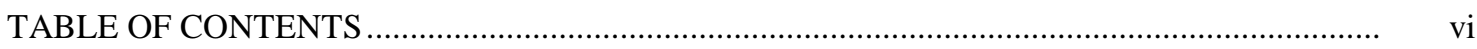

LIST OF FIGURES......................................................................................................... viii

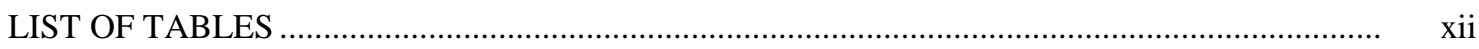

\section{CHAPTER}

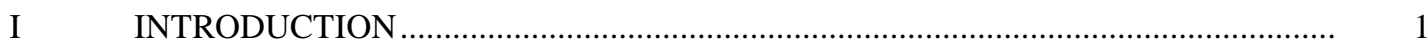

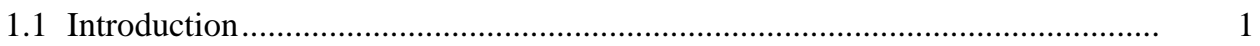

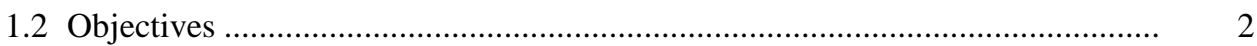

1.3 Statement of the Problem ...................................................................................... 2

1.4 Validation and Application ............................................................................... 4

1.5 A Parametric Correlation Study ……………................................................ 10

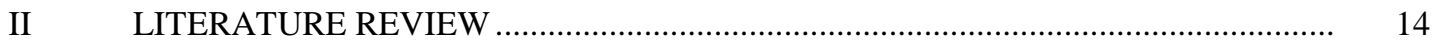

2.1 Classical Time-Rate Analysis ........................................................................... 14

2.2 Modern Time-Rate Analysis ............................................................................ 16

III NEW RATE DECLINE RELATIONS AND DIAGNOSTICS …………........................ 21

3.1 Modified Wiorkowski Model............................................................................... 21

3.2 Modified Ilk Model ........................................................................................ 23

3.3 Time-Cumulative Diagnostics ...................................................................... 24

3.4 Validation - Synthetic \& Field Examples .......................................................... 25

IV PARAMETRIC CORRELATIONS OF WELL/RESERVOIR PROPERTIES AND PRODUCTION METRICS …………………….............................................. 51

4.1 General Methodology ................................................................................. 51

4.2 Fracture Conductivity - Parametric Correlations............................................... 52

4.3 Fracture Half Length - Parametric Correlations ................................................ 64

4.4 Formation Permeability - Parametric Correlations .............................................. 75 
V SUMMARY, CONCLUSIONS AND

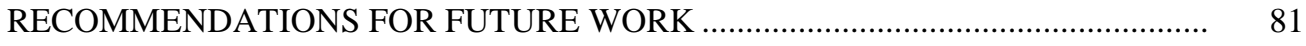

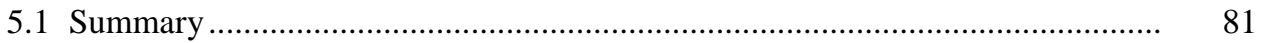

5.2 Conclusions .................................................................................. 82

5.3 Recommendation for Future Work .......................................................... 82

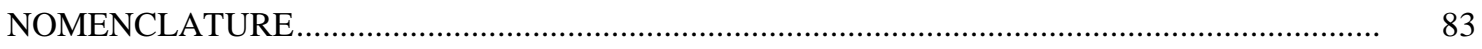

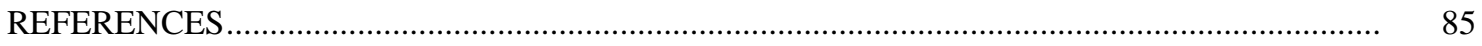

APPENDIX A - MODIFIED WIORKOWSKI MODEL .......................................................

APPENDIX B - MODIFIED ILK MODEL ....................................................................

APPENDIX C - DEVELOPMENT OF TIME-CUMULATIVE PRODUCTION DIAGNOSTICS.. 94 APPENDIX D - A METHODOLOGY TO REDUCE UNCERTAINTY IN

EUR ESTIMATION FOR MODIFIED WIORKOWSKI MODEL ....................... 98

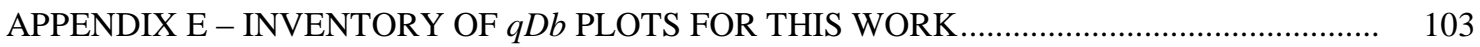




\section{LIST OF FIGURES}

1.1 (Cartesian Plot): Production history plot for numerical simulation case - flow rate,(MSCFD) and cumulative production, (MSCF) versus production time,(Days) 6

1.2 (Log-log Plot): (Log-log Plot): Flow rate $\left(q_{g}\right), D$ - and $b$-parameter versus production time. PLE, Logistic growth, Duong, MWM and MIM time-rate model matches for numerical simulation case.

1.3 (Cartesian Plot): EUR estimates from Modified Wiorkowski and Modified Ilk Models and $G_{p, \max }$ projected from numerical simulation case 9

1.4 Diagram of the numerical simulation model showing horizontal well and multiple transverse fractures.

1.5 Comparison of fracture conductivity and 30 year EUR values calculated using parametric correlations developed using the Modified Wiorkowski Model para-meters versus results from model-based production analysis (or RTA)

1.6 Comparison of fracture half-length and 30 year EUR values calculated using parametric correlations developed using the Modified Wiorkowski Model para-meters versus results from model-based production analysis (or RTA)

1.7 Comparison of formation permeability and 30 year EUR values calculated using parametric correlations developed using the Modified Wiorkowski Model para-meters versus results from model-based production analysis (or RTA)

2.1 (Log-log Plot): Flow rate $\left(q_{g}\right)$ and $D$-parameter versus production time- Hyperbolic and power law exponential rate decline and loss ratio models illustration for orientation purposes (SPE 116731).

2.2 (Log-log Plot): Material-Balance time characteristic plots for Numerical Simulation Case - material-balance time,(Days) and inverse material-balance time, (1/Days) versus production time,(Days).

3.1 (Log-log Plot) Schematic example of time-rate plotting functions for a horizontal gas well with multiple transverse fractures (numerical simulation case).

3.2 (Cartesian Plot): Production history plot for numerical simulation case - flow rate,(MSCFD) and cumulative production, (MSCF) versus production time,(Days)

3.3 (Log-log Plot): Flow rate $\left(q_{g}\right), D$ - and $b$-parameter versus production time. PLE, Logistic growth, Duong, MWM and MIM time-rate model matches for numerical simulation case..

3.4 (Cartesian Plot): EUR estimates from Modified Wiorkowski and Modified Ilk Models and $\mathrm{G}_{p}$,max projected from numerical simulation case.

3.5 (Semi-log Plot): Production history plot for East Texas Tight Gas Well - flow rate,(MSCFD) and cumulative production, (BSCF) versus production time,(Days) .32 
3.6 (Log-log Plot): " $q D b "$ plot — Flowrate $\left[q_{g}(t)\right]$, Arps' Inverse Loss Ratio $[D(t)]$, and Arps' Loss Ratio Derivative $[b(t)]$ versus production time. The Modified-Wiorkowski Model, Modified-Ilk Model, Arps' Hyperbolic Model, Duong Model, Power Law Exponential Model, and the Logistic Growth Model diagnostic function matches for the East Texas Tight Gas Well.

3.7 (Log-log Plot): Modified Wiorkowski Model, Modified Ilk Model, Arps’ Hyperbolic Model, Duong Model, Power Law Exponential Model and Logistic Growth Model 30year EUR forecasts for East Texas Tight Gas Well -flow rate,(MSCFD) and cumulative production, (MSCF) versus production time,(Days).

3.8 (Semi-log Plot): East Texas Tight Gas Well analysis summary plot - flow rate,(MSCFD) and calculated bottomhole pressure, (psia) versus production time,(Days)

3.9 (Cartesian Plot): "Continuous EUR" obtained using the Modified-Wiorkowski and Modified-Ilk models compared to the cumulative production (East Texas Gas Well).

3.10 (Semi-log Plot): Production history plot for Well-4 - flow rate,(MSCFD) and cumulative production, (BSCF) versus production time,(Days)

3.11 (Log-log Plot): " $q D b "$ plot — Flowrate $\left[q_{g}(t)\right]$, Arps' Inverse Loss Ratio $[D(t)]$, and Arps' Loss Ratio Derivative $[b(t)]$ versus production time. The Modified-Wiorkowski Model, Modified-Ilk Model, Arps' Hyperbolic Model, Duong Model, Power Law Exponential Model, and the Logistic Growth Model diagnostic function matches for the Marcellus Shale Gas Well (Well-4). $G_{p, \max }$ of East TX tight gas well.

3.12 (Log-log Plot): Modified-Wiorkowski Model, Modified-Ilk Model, Arps' Hyperbolic Model, Duong Model, Power Law Exponential Model and Logistic Growth Model 30year EUR forecasts for the Marcellus Shale Gas Well (Well-4) — gas flowrate (MSCFD) and cumulative gas production (BSCF) versus production time (Days).

3.13 (Cartesian Plot): "Continuous EUR" obtained using the Modified-Wiorkowski and Modified-Ilk models compared to the cumulative production (Marcellus Shale Gas Well (Well-4)).

3.14 (Semi-log Plot): Well-4 analysis summary plot -flow rate,(MSCFD) and calculated bottomhole pressure, (psia) versus production time,(Days) ...

3.15 (Semi-log Plot): Production history plot for Eagle Ford Gas Well - flow rate,(MSCFD) and cumulative production, (BSCF) versus production time,(Days)

3.16 (Log-log Plot): Flow rate, $D$ - and $b$-parameter versus production time. Modified Wiorkowski Model and Modified Ilk Model matches for Eagle Ford Gas Well

3.17 (Log-log Plot): Modified-Wiorkowski Model, Modified-Ilk Model, Arps' Hyperbolic Model, Duong Model, Power Law Exponential Model, and the Logistic Growth Model time-rate model matches for the Eagle Ford Gas Well case — gas flowrate (MSCFD) and cumulative gas production (BSCF) versus production time (Days).

3.18 (Semi-log Plot):Eagle Ford Gas Well analysis summary plot -flow rate, (MSCFD) and calculated bottomhole pressure, (psia) versus production time,(Days) .50 
4.1 Diagram of the numerical simulation model showing horizontal well and multiple transverse fractures.

4.2 (Log-log Plot): Flow rate $\left(q_{g}\right)$ versus production time. Modified Wiorkowski model matches of 15 numerical simulation cases

4.3 (Log-log Plot): $b$-parameter versus production time. Modified Wiorkowski model matches of 15 numerical simulation cases

4.4 (Log-log Plot): $D$ - parameter versus production time. Modified Wiorkowski model matches of 15 numerical simulation cases.

4.5 Cross-plots showing relationship between Modified Wiorkowski model parameters and numerical simulation case fracture conductivity $\left(F_{c}\right)$ and 30 year EUR estimates.

4.6 Comparison of fracture conductivity and 30 year EUR values calculated using parametric correlations developed using Modified Wiorkowski model parameters versus numerical simulation models

4.7a (Log-log Plot): Flow rate $\left(q_{g}\right)$ versus production time. Modified Ilk matches of 15 numerical simulation cases.

4.7b (Log-log Plot): $b$-parameter versus production time. Modified Ilk model matches of 15 numerical simulation cases.

4.8 (Log-log Plot): $D$-parameter versus production time. Modified Ilk model matches of 15 numerical simulation cases.

4.9 Cross-plots showing relationship between Modified Ilk model parameters and numerical simulation cases fracture conductivity $\left(F_{c}\right)$ and 30 year EUR values.

4.10 Comparison of fracture conductivity and 30 year EUR values calculated using parametric correlations developed using Modified Ilk model parameters versus numerical simulation models.

4.11 (Log-log Plot): Flow rate $\left(q_{g}\right)$ versus production time. Modified Wiorkowski model matches of 15 numerical simulation cases with varying fracture half-lengths

4.12 (Log-log Plot): $b$-parameter versus production time. MWM model matches of 15 numerical simulation cases with varying fracture half-lengths

4.13 (Log-log Plot): $D$-parameter versus production time. MWM model matches of 15 numerical simulation cases with varying fracture half-lengths.

4.14 Cross-plots showing relationship between Modified Wiorkowski model parameters and numerical simulation case fracture half-length $(x f)$ and 30 year EUR estimates.

4.15 Comparison of fracture half-length and 30 year EUR values calculated using parametric correlations developed using Modified Wiorkowski model parameters versus numerical simulation models

4.16 (Log-log Plot): Flow rate $\left(q_{g}\right)$ versus production time. Modified Ilk matches of 15 numerical simulation cases with varying fracture half-lengths. 
4.17 (Log-log Plot): $b$-parameter versus production time. Modified Ilk model matches of 15 numerical simulation cases with varying fracture half-lengths.

4.18 (Log-log Plot): $D$ - parameter versus production time. Modified Ilk model matches of 15 numerical simulation cases with varying fracture half-lengths.

4.19 Cross-plots showing relationship between Modified Ilk model parameters and numerical simulation cases fracture half-length $(x f)$ and 30 year EUR values

4.20 Comparison of fracture half-length and 30 year EUR values calculated using parametric correlations developed using Modified Ilk model parameters versus numerical simulation models.

4.21 (Log-log Plot): Flow rate $\left(q_{g}\right)$ versus production time. Modified Ilk matches of 15 numerical simulation cases with varying formation permeability.

4.22 (Log-log Plot): $b$-parameter versus production time. Modified Ilk model matches of 15 numerical simulation cases with varying formation permeability.

4.23 (Log-log Plot): D-parameter versus production time. Modified Ilk model matches of 15 numerical simulation cases with varying formation permeability.

4.24 (Cross-plots showing relationship between Modified Ilk model parameters and numerical simulation cases formation permeability $(k)$ and 30 year EUR values

4.25 Comparison of formation permeability and 30 year EUR values calculated using parametric correlations developed using Modified Ilk model parameters versus numerical simulation models. 


\section{LIST OF TABLES}

TABLE

1.1 Reservoir and fluid properties for numerical simulation case (horizontal well with multiple transverse fractures).

1.2 PLE, Duong, Logistic growth model and newly derived time-rate relations. 6

1.3 Modified Wiorkowski Model matched parameters for Numerical Simulation case ....................7

1.4 Modified Ilk Model matched parameters for Numerical Simulation case ..................................

1.5 Summary of Decline Curve Analyses (EUR) for the Numerical Simulation Case. $(G p, \max =1.92 \mathrm{BSCF}$ at 30 years from numerical simulation.)...............................................

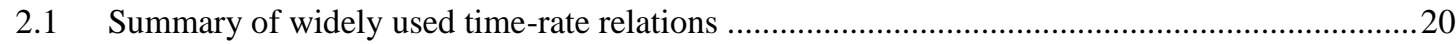

3.1 Reservoir and fluid properties for numerical simulation case (horizontal well with

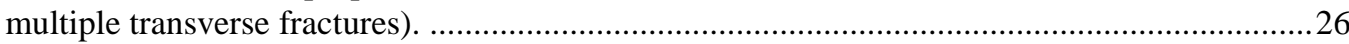

3.2 PLE, Duong, logistic growth model and newly derived time-rate relations. ................................27

3.3 Summary of Decline Curve Analyses (EUR) for the Numerical Simulation Case. $(G p, \max =1.92 \mathrm{BSCF}$ at 30 years from numerical simulation.) ...................................................29

3.4 Reservoir and fluid properties for East Texas Tight Gas Well .................................................... 31

3.5 Modified Wiorkowski and Modified Ilk Models matched parameters for East Texas

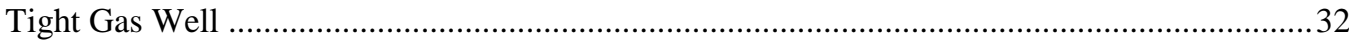

3.6 Summary of Decline Curve Analyses (EUR) for the East Texas Tight Gas Well.

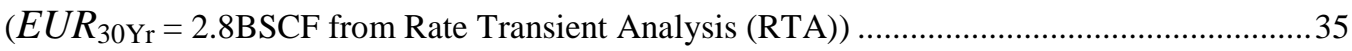

3.7 Reservoir and fluid properties for Marcellus Shale Gas Well-4 ………………………..............35

3.8 Modified Wiorkowski and Modified Ilk Models matched parameters for Well-4 .......................38

3.9 Arps' Exponential, Arps' Hyperbolic, Duong, Logistic growth, Power Law and Wiorkowski Model matched parameters for Well-4.......................................................................39

3.10 Reservoir and fluid properties for Eagle Ford Gas Well …….................................................... 43

3.11 Modified Wiorkowski and Modified Ilk Models matched parameters Eagle Ford Gas Well .

3.12 Summary of Decline Curve Analyses (EUR) for the Eagle Ford Gas Well case...........................46

3.13 Arps' Exponential, Arps' Hyperbolic, Duong, Logistic growth, Power Law and Wiorkowski Model matched parameters for Eagle Ford Gas Well. .48

3.14 Summary of Decline Curve Analyses (EUR) for the Numerical Simulation Case. ( $G p, \max =1.92 \mathrm{BSCF}$ at 30 years from numerical simulation.) ...... 
4.1 Reservoir and fluid properties for numerical simulation case (horizontal well with multiple transverse fractures) with varying fracture conductivity.

4.2 Modified Wiorkowski Model parameters. Model matches to 15 numerical simulation

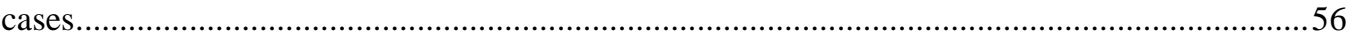

4.3 Modified Ilk Model parameters. Model matches to 15 numerical simulation cases..................61

4.4 Reservoir and fluid properties for numerical simulation case (horizontal well with multiple transverse fractures) with varying fracture half-lengths.

4.5 Modified Wiorkowski Model parameters. Model matches to 15 numerical simulation cases with varying fracture half-lengths

4.6 Modified Ilk Model parameters. Model matches to 15 numerical simulation cases with varying fracture half-lengths.

4.7 Reservoir and fluid properties for numerical simulation case (horizontal well with multiple transverse fractures) with varying formation permeability.

4.8 Modified Ilk Model parameters. Model matches to 15 numerical simulation cases with varying formation permeability. 


\section{CHAPTER I}

\section{INTRODUCTION}

\subsection{Introduction}

The origin of "decline curve analysis" (i.e., the estimation of ultimate producible volumes from production curves) appears to be from the "Manual for the Oil and Gas Industry" written as part of the Revenue Act of 1918 by the US Internal Revenue Service (1919). Initially, only graphical extrapolations of production trends were presented, but the fundamental concept of using the "area under the curve" for estimated ultimate recovery is evident in this work. This work presents the development of two new rate-time relations which are based on self-growth limiting time-cumulative relations.

Modified Wiorkowski Model is inspired from the Wiorkowski cumulative production model (1981), which is a variant of the Richards family of "statistical" growth curves. Wiorkowski was trying to develop statistically rigorous models that can be used to ultimate oil production volumes from continental US. We propose a simplified form of the Wiorkowski model below. This model and the associated functions derived from it are presented in Appendix A.

$$
G_{p}(t)=\tilde{G}_{p}\left(1-\tilde{a} \exp \left[-\tilde{D}_{i} t\right]\right)^{\tilde{n}}
$$

Based on the empirical observation of power-law (straight line) behavior of Arps' inverse loss ratio for early-time and transitional flow regimes in wells from low/ultra-low permeability reservoirs, Ilk, et al. (2008) formulated Power Law Exponential (PLE) rate-time relation. We propose an intuitive integral form of PLE as below. This model and the associated functions derived from it are presented in Appendix B.

$$
G_{p}(t)=\bar{G}_{p}\left(1-\exp \left[-\bar{D}_{\infty} t-\bar{D}_{i} t^{\bar{n}}\right]\right)
$$

We also explore the utility of diagnostics that are based on growth character of historical cumulative production data. We observed that these diagnostics provide characteristics that are less influenced by the noise that are ever-present in oil field data. Furthermore, we show that definitions of these diagnostics help us formulate special plotting relations for proposed Modified Wiorkowski Model, which can be used to determine regression parameters directly from historical production data, thereby reducing uncertainty.

We validate our proposed relations against existing rate-time relations (i.e., the Arps Exponential decline, the Arps Hyperbolic decline, the PLE, the Duong, and the Logistic Growth models) and we test their application for a number of well/reservoir configurations. 
Finally, we present a study to integrate time-rate model parameters with fundamental completion and reservoir properties (i.e., fracture conductivity $\left(F_{c}\right)$, fracture half length $\left(\mathrm{x}_{\mathrm{f}}\right)$, formation permeability $(\mathrm{k})$ and 30 year $\left.E U R\left(E U R_{30 \mathrm{yr}}\right)\right)$ using parametric correlations. Previously, Ilk et al. (2011) and Askabe (2012) have shown that it is possible to correlate reservoir/well properties that are estimated using model-based production data analysis with model parameters of time-rate relations. We demonstrated the application of a methodology that allows formulating multivariate parametric correlations to integrate completion and reservoir parameters with time-rate model parameters.

For this study we considered a number of cases for a low permeability horizontal well with multiple transverse factures. The correlations developed in this work allow the estimation of completion/reservoir properties from time-rate model parameters (with the help of benchmark results). We investigate parametric correlations for the Modified Wiorkowski Model and the Modified Ilk Model for fracture conductivity, formation permeability, fracture half-length and 30-year EUR values.

\subsection{Objectives}

The objectives of this work are to:

- To propose two new rate-time relations that are based on self-limiting growth functions.

- To propose cumulative production diagnostics for flow data characterization which are analogous to decline diagnostics as proposed by Johnson and Bollens (1928) and later by Arps (1945).

- To demonstrate the applicability and comparison of proposed rate-time relations with existing ratetime relations for selected cases.

- To develop a methodology for integration of reservoir/well properties - specifically, to demonstrate the correlation of fracture conductivity, formation permeability, fracture half-length and 30 year EUR estimate $\left(E R_{30 y r}\right)$ with time-rate model parameters, using production data generated from numerical simulation models.

\subsection{Statement of the Problem}

Decline Curve Analysis techniques have been employed by petroleum engineers since the introduction of the Manual for the Oil and Gas Industry under the Revenue Act of 1918 by the US Internal Revenue Service (1919), to estimate oil and gas reserves. Their widespread applicability and acceptance is due to their simplicity and simple formulations. However, historical time-rate relations are empirical in nature and are only (strictly) applicable to the boundary-dominated flow regime. Production forecasts from these models assume that the well continues production with same operating conditions as that of the past 
production history. Alternatively, several statistical models have been employed since the 1950s to determine total amount of recoverable hydrocarbons. The basic aim of these studies was to estimate remaining reserves in the conterminous U.S. and the U.S. outer continental shelf. These efforts employed both decline (in discoveries and yearly volumes) and growth in cumulative production.

All rate-time relations currently being used for estimation of reserves face the problem of unconstrained growth of reserves. The traditional Arps' relations are based on "loss-ratio" and the "derivative of the lossratio" functions as proposed by Johnson and Bollens (1928) and later by Arps (1945) are strictly valid in conventional reservoirs for boundary-dominated flow.

Definition of the Loss-Ratio:

$$
\frac{1}{D} \equiv-\frac{q}{d q / d t}
$$

Derivative of the Loss-Ratio:

$$
b \equiv \frac{d}{d t}\left[\frac{1}{D}\right] \equiv-\frac{d}{d t}\left[\frac{q}{d q / d t}\right] .
$$

Through time, these functions have been designated as the "Arps" relations due to the presentation and use of these relations in the seminal paper by Arps (1945). These functions were used to develop the traditional Arps' time-rate relations. On a practical note, when the traditional Arps' hyperbolic time-rate and time-cumulative relations are used to forecast production and to estimate reserves, these relations typically overestimate the performance for low to ultra-low permeability reservoirs. For reference, the Arps' hyperbolic time-rate and time-cumulative relations are:

Arps' Hyperbolic Time-Rate Model:

$$
q(t) \equiv \frac{q_{i, h y p}}{\left(1+b D_{i} t\right)^{1 / b}}
$$

Arps' Hyperbolic Time-Cumulative Model:

$$
G_{p} \equiv \frac{q_{i, h y p}}{(1-b) D_{i}}\left[1-\left(1+b D_{i} t\right)^{1-1 / b}\right]
$$

For the case of wells in low to ultra-low permeability reservoirs, the performance of these wells is characterized by a very long transient flow regime, which often dominates most of the wells productive life (as much as 10-15 years, or more). Often times, the traditional boundary-dominated flow regime is not observed in production data from wells in ultra-low (unconventional) reservoirs. In such cases, the 
incorrect application of the Arps' relations (Eqs. 1.5 and 1.6) to performance data for these wells often yields overestimation of reserves.

The issue is that of the "Arps" $b$-parameter (or the "loss-ratio derivative,") which is typically on the order of 2 (rather than $b<1$ as the traditional (boundary-dominated flow) assumption) - we note that a $b$ parameter of 2 corresponds (as a coincidence) to the "linear flow" regime which exists for cases where the fractures in an multi-fractured horizontal well (MFHW) have pressure distributions that do not interfere. As is well known in the pressure transient testing literature, the linear flow regime depends on the properties of the hydraulic fractures as well as the permeability of the formation.

Various modern rate-time relations have been proposed (Ilk et al 2008, Valko 2009, Clark et al 2011, Mishra 2012, etc.) that address this unbounded reserves problem. These models result in better matches for low and ultra-low permeability wells with a very long transient and transition flow regime (Askabe et al 2012). Ilk et al. (2008) observed that $D$-parameter exhibits a power law behavior for early time data. This is characterized by a straight line behavior on $\log \log$ plot of $D$ - parameter versus time. During late times, the PLE model can yield a constant (i.e., exponential) decline and, thus, can match transient, transition and boundary-dominated flow regimes.

Furthermore, all proposed rate-time relations present the problem of non-uniqueness, where reasonable matches to historical production data can be obtained for more than one set of regression parameter combinations. This results in substantial uncertainty in long time forecasts of time-rate data and can lead to significant variations in EUR estimates. All time-rate relations that involve regression of more than two parameters experience this problem. Some efforts have been made to remove non-linearity from parameter matching process by proposing diagnostic plots (Clark et al. 2011, Mishra 2012), which allow estimation of model parameters directly from field data character using graphical parameters such as the slope and intercept on a specific plot. However, these methods still involve a prior knowledge of "Carrying Capacity" or "Initial-Gas-In-Place." Therefore, a need arises to remove non-linearities from the model matching process so that regression parameters can be estimated from data character, which reduces uncertainty in production forecasts.

\subsection{Validation and Application}

In this section, we demonstrate the performance analysis of the Modified Wiorkowski Model (MWM) and the Modified Ilk Model(MIM) using a numerical simulation case. A scheme is presented to allow direct parameter estimation for the Modified Wiorkowski Model from historical production data through a specialized plot, thereby eliminating non-linearities and reducing uncertainty in the model matching process (this work is presented in Appendix D). However; it should be noted that this technique does not yield reasonable estimates of parameters for any field case data with regular noise and distortion (because 
of depending on first and second order derivative parameters of gas rate) and thus should be further improved for actual field data cases. We perform a model match on a diagnostic plot and compare the EUR estimates of proposed new models and existing time-rate relations. Finally we will introduce the parametric correlation study.

Numerical Simulation Case: Synthetic low permeability well

For this numerical simulation case, we consider a low permeability horizontal well with multiple transverse fractures and we have generated a production profile for 10,000 days ( 30 years) as a means of validating the $E U R_{30 Y r}$ (estimated ultimate recovery at 30 years). The well is produced at a constant bottomhole pressure of 1,000 psia. The model input parameters are provided in Table 1.1. The historical flow rate and cumulative production data is shown in Fig. 1.1.

Table $1.1-$ Reservoir and fluid properties for numerical simulation case (horizontal well with multiple transverse fractures).

Reservoir Properties

Net pay thickness, $h=200 \mathrm{ft}$

Formation permeability, $k=1 \times 10^{-4} \mathrm{md}$

Fracture conductivity, $F_{c D}=$ infinite

Wellbore Radius, $r_{w}=0.25 \mathrm{ft}$

Formation compressibility, $c_{f}=3 \times 10^{-6} \mathrm{psia}^{-1}$

Porosity, $\phi=0.05$ (fraction)

Initial reservoir pressure, $p_{i}=5,000 \mathrm{psia}$

Gas saturation, $s_{g} \quad=\quad 0.65$ fraction

Skin factor, $s \quad=0$ (dimensionless)

Reservoir temperature, $T_{r}=212^{\circ} \mathrm{F}$

Fluid properties:

Gas specific gravity, $\gamma_{g} \quad=\quad 0.6($ air $=1)$

Hydraulically fractured well model parameters:

Fracture half-length, $x_{f} \quad=\quad 200 \mathrm{ft}$

Number of fractures $\quad=\quad 100$

Horizontal well length, $L_{h} \quad=\quad 5,000 \mathrm{ft}$

Production parameters:

Last tubing pressure, $p_{w f}=1,000$ psia

Production time, $t \quad=\quad 10,000$ days $(\sim 30$ years $)$ 


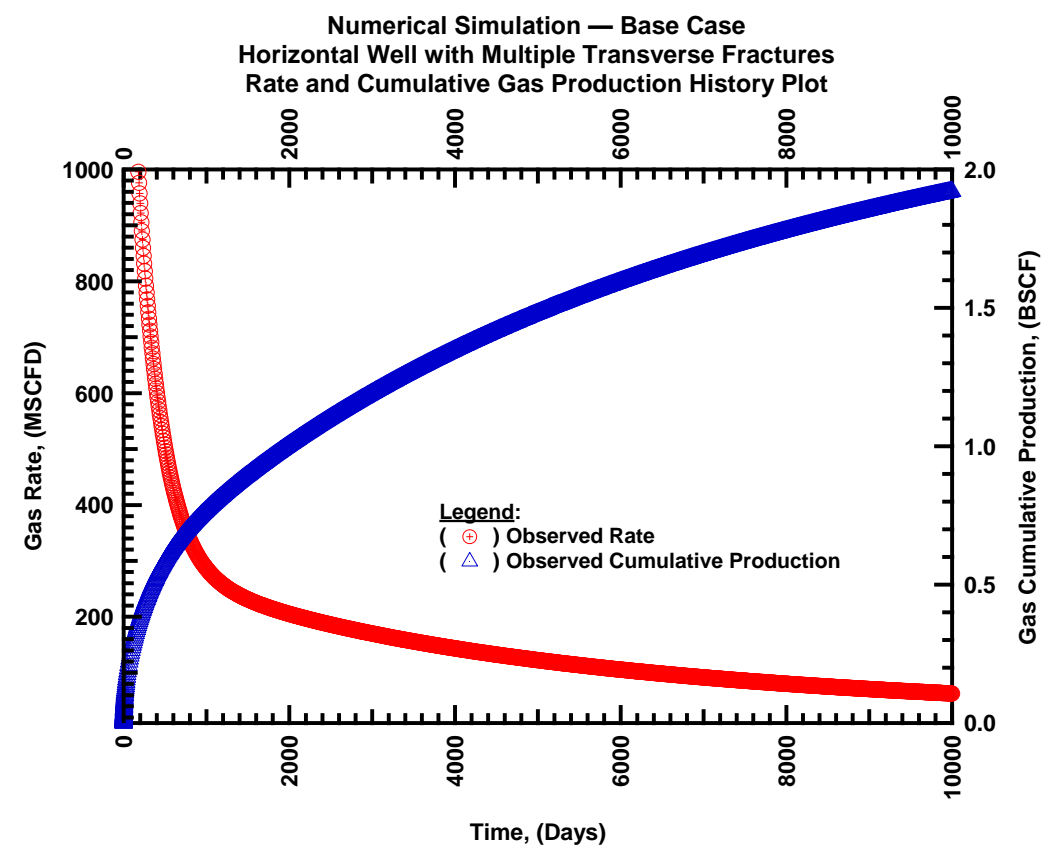

Figure $1.1-\quad$ (Cartesian Plot): Production history plot for numerical simulation case - flow rate,(MSCFD) and cumulative production, (MSCF) versus production time,(Days)

A summary of time-rate relations of all models used for this comparative study are presented in Table.

1.2. The table also shows the newly derived time-rate relations.

Table 1.2 - PLE, Duong, logistic growth model and newly derived time-rate relations.

\begin{tabular}{|c|c|}
\hline Time-Rate Relations & Models \\
\hline Power-Law Exponential Model & $q(t)=\hat{q}_{g i} \exp \left[-D_{\infty} t-\hat{D}_{i} t^{n}\right]$ \\
\hline Duong Model & $q(t)=q_{1} t^{-m} \exp \left[\frac{a}{1-m}\left(t^{1-m}-1\right)\right], q_{1}$ at $t=1$ \\
\hline Logistic Growth Model & $q(t)=\frac{\hat{a} K \hat{n} t^{\hat{n}-1}}{\left(\hat{a}+t^{\hat{n}}\right)^{2}}$ \\
\hline Arps' Hyperbolic Model & $q(t)=\frac{\hat{q}_{g i}}{\left(1+D_{i} b t\right)^{1 / b}}$ \\
\hline Arps' Exponential Model & $q(t)=\hat{q}_{g i} \exp \left[-D_{i} t\right]$ \\
\hline New Time-Rate Relations & Models \\
\hline Modified Wiorkowski Model & $q(t)=\tilde{G}_{p} \tilde{D}_{i} \tilde{a} \tilde{n} \exp \left[-\tilde{D}_{i} t\right]\left(1-\tilde{a} \exp \left[-\tilde{D}_{i} t\right]\right)^{\tilde{n}-1}$ \\
\hline Modified Ilk Model & $q(t)=\bar{G}_{p} \exp \left[-\bar{D}_{\infty} t-\bar{D}_{i} t^{\bar{n}}\right]\left(\bar{D}_{\infty}+\bar{n} \bar{D}_{i} t^{\bar{n}-1}\right)$ \\
\hline
\end{tabular}


We next determine the parameters for the Modified Wiorkowski Model from historical production data directly. This will enable us to determine EUR by analyzing character of historical data - the methodology to determine the time-rate parameters for the Modified Wiorkowski Model using a specialized plot is presented in Appendix D

The matched parameters are:

Table $1.3 \quad$ Modified Wiorkowski Model matched parameters for Numerical Simulation case

\begin{tabular}{cc} 
Parameter & Simulation Case \\
\hline$\tilde{G}_{p}$ & $2.60 \mathrm{E}+06$ \\
$\tilde{a}$ & 0.999 \\
$\widetilde{D}_{i}$ & 0.0001 \\
$\tilde{n}$ & 0.56 \\
\hline
\end{tabular}

Modified Ilk Model was graphically fitted to historical production data of numerical simulation case. Regressed parameters are given in Table. 1.4.

Table $1.4 \quad$ Modified Ilk Model matched parameters for Numerical Simulation case.

\begin{tabular}{cc} 
Parameter & Simulation Case \\
\hline $\bar{G}_{p}$ & $3.1 \times 10^{6}$ \\
$\bar{D}_{\infty}$ & $9.5 \times 10^{-3}$ \\
$\bar{D}_{i}$ & 0.015 \\
$\bar{n}$ & 0.35 \\
\hline
\end{tabular}

In Fig. 1.2, we present the "qDb-plot" plot for this case. On the $q D b$-plot the following functions are plotted - flowrate $\left[q_{g}(t)\right]$, Arps' Inverse Loss Ratio $[D(t)]$, and Arps' Loss Ratio Derivative $[b(t)]$ versus production time. In this case we plot the $D(t)$ and $b(t)$ data functions (symbols) as well as the $D(t)$ and $b(t)$ model functions (lines) for the Power-Law Exponential (PLE) model, the Logistic Growth Model (LGM), the Duong model, the Modified-Wiorkowski model (MWM), and the Modified-Ilk model (MIM). 


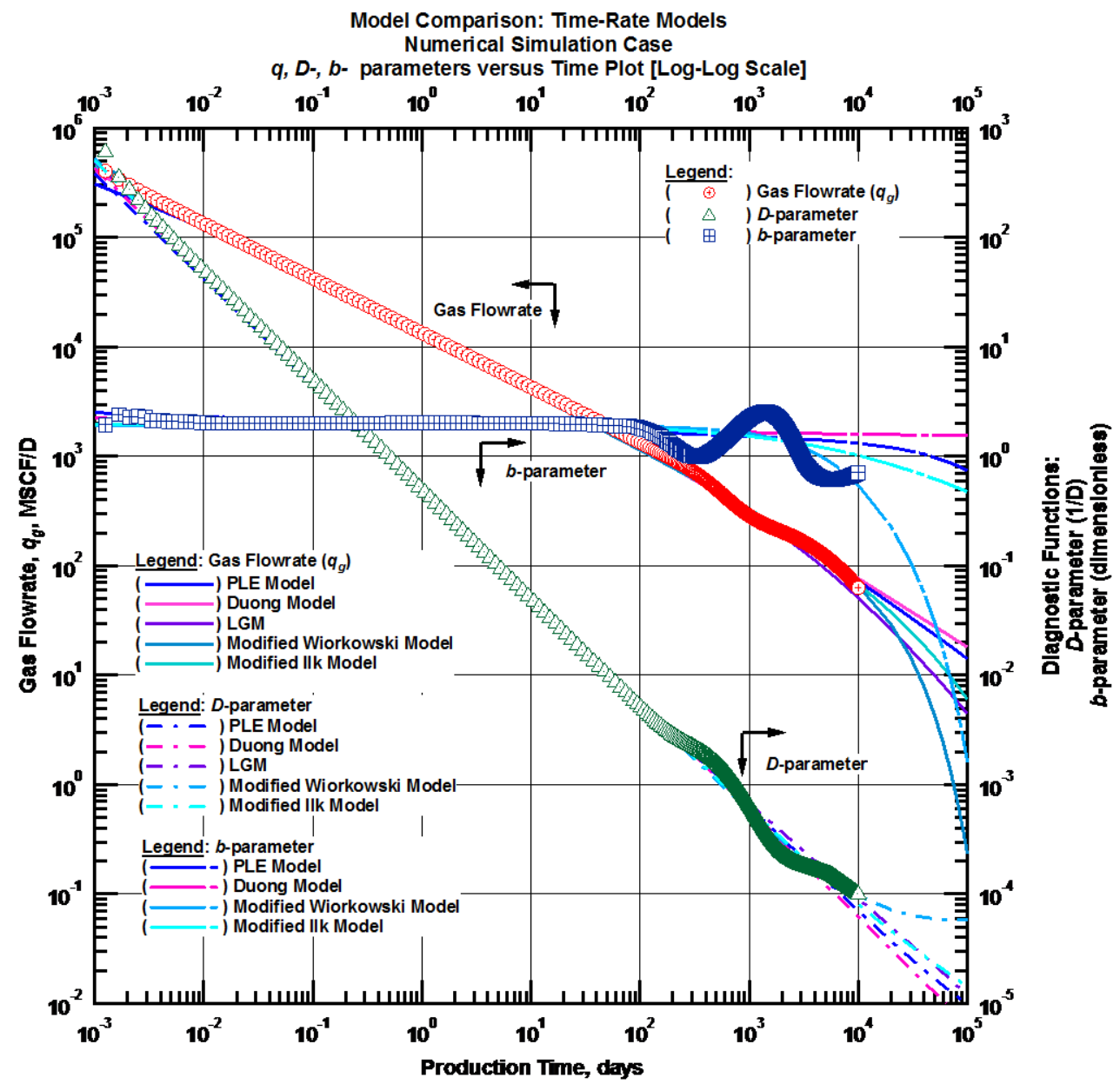

Figure $1.2-\quad\left(\log -\log\right.$ Plot): Flow rate $\left(q_{g}\right), D$ - and $b$-parameter versus production time. PLE, Logistic growth, Duong, MWM and MIM time-rate model matches for numerical simulation case.

In Figure 1.2, it can be observed that each model gives reasonable rate and cumulative matches. Moreover, the Modified Wiorkowski Model (MWM) along with the Modified Ilk Model(MIM) yield the most conservative estimates of 30-year EUR for this numerical simulation case. This can be attributed to selflimiting growth nature of both the MWM and MIM models, which results in inherently conservative matches across the transient, transition and boundary-dominated flow regimes. The $D(t)$ and $b(t)$ data functions do capture the end of linear flow observed in the flow rate data at about 700 days, but the later transition and apparent boundary-dominated flow regimes are not so clearly defined. In fact, focusing solely on the $D(t)$ data function, we note that this function suggests essentially only a single trend (i.e., all of the data shown by the green symbols could, in a practical sense, be captured by a single power-law, straight-line trend). 
Table 1.5 provides a summary of the results for all of the time-rate decline model applied to the example numerical simulation case. We note from Fig. 1.2 that all of the matches are reasonable. However the Modified-Wiorkowski Model (MWM) and the Modified-Ilk Model (MIM) model yield the most conservative estimates of 30-year EUR (i.e., EUR30Yr). We believe that this "conservative" behavior is attributed to the self-limiting growth capability of both the MWM and MIM models.

Table $1.5-$ Summary of Decline Curve Analyses (EUR) for the Numerical Simulation Case. (Gp, max $=1.92 \mathrm{BSCF}$ at 30 years from numerical simulation.)

\begin{tabular}{lll} 
Time-rate models & & $G_{p, \max }$ \\
\cline { 1 - 1 } Duong model & & $2.70 \mathrm{BSCF}$ \\
Logistic growth model & & $2.49 \mathrm{BSCF}$ \\
Power-law exponential model (PLE) & & $2.74 \mathrm{BSCF}$ \\
Arps' Hyperbolic Model & & $2.89 \mathrm{BSCF}$ \\
Modified Wiorkowski Model & & $1.79 \mathrm{BSCF}$ \\
Modified Ilk Model & $1.93 \mathrm{BSCF}$
\end{tabular}

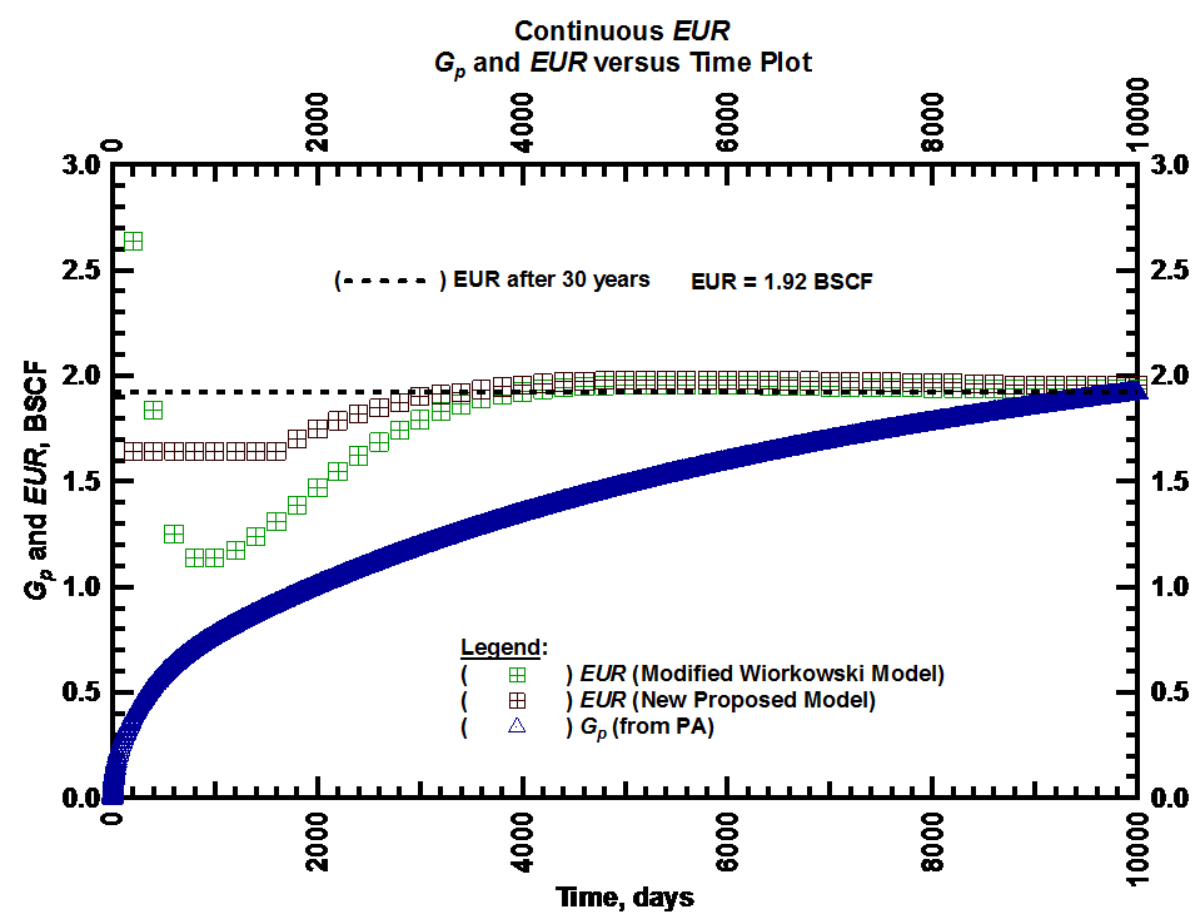

Figure 1.3 - (Cartesian Plot): EUR estimates from Modified Wiorkowski and Modified Ilk Models and $\mathrm{G}_{p, \max }$ projected from numerical simulation case.

In Fig. 1.3 we show the "Continuous EUR" results versus production time for the MWM and MIM models. For the numerical simulation case we observe convergence to the 30-year EUR (actually the gas 
produced at 30 years since this is a simulation case) at around 3,000 days (or approximately 10 years), which is consistent with expectations for a multi-fracture horizontal well (MFHW) producing in an ultralow permeability formation. We do note that the behavior of the MWM and MIM models on the "Continuous EUR" plot is unique in our experience. Most models converge from above, indicating that reserves are reduced over time, these models converge from below, which indicates that these models increase reserves with time - which is a desirable aspect of a reserves prediction tool.

\subsection{A Parametric Correlation Study}

In this section, we present a methodology to integrate parameters from our proposed time-cumulative relations with completion/reservoir parameters derived from model-based production analysis (also known as "Rate Transient Analysis" (or RTA)). We develop parametric correlations for the Modified Wiorkowski Model and the Modified Ilk Model for fracture conductivity $\left(F_{c}\right)$, formation permeability $(k)$, fracture half-length $\left(x_{f}\right)$ and the 30-year EUR $\left(\mathrm{EUR}_{30 \mathrm{Yr}}\right)$ values. We provide a theoretical consideration for this methodology using data generated from several different numerical simulation cases for a horizontal well with multiple transverse fractures in a low/ultra-low permeability reservoir.

We model a horizontal well with multiple transverse fractures in a low permeability reservoir for numerical simulation cases. We make a number of numerical simulation runs for varying fracture conductivity, formation permeability and fracture half-length while keeping all other factors constant. A typical numerical simulation grid is shown in Fig. 1.4.

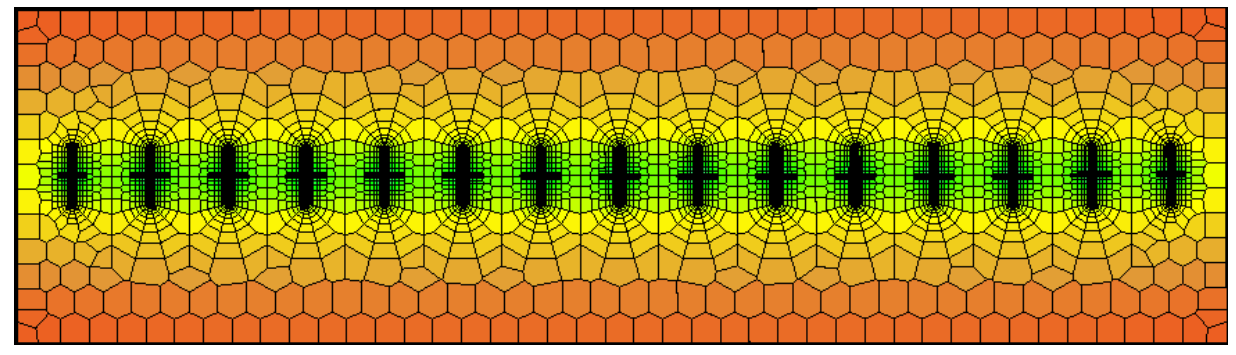

Figure 1.4 - Diagram of the numerical simulation model showing horizontal well and multiple transverse fractures.

For each case we make 15 simulation runs and perform time-rate analysis with the Modified Wiorkowski Model(MWM) and the Modified Ilk Model(MIM). We use the Bourdet algorithm (Bourdet et al. 1989) to calculate the $D(t)$ - and $b(t)$-parameters to help in matching the production data. We generate log-log plots of gas rate $q_{g}(t), D(t)$ - and $b(t)$-parameters against production time (i.e., the "qDb" plots) to inspect the quality of our model matches. 
Once we obtain satisfying matches using time-rate models, our next step is to study the relationship between individual model parameters and the reservoir parameter being considered along with 30 year EUR. We perform a cross-plot analysis of the individual rate decline model parameters with reservoir parameters and identify respective correlating parametric functions.

We intend to find each reservoir parameter in terms of the rate decline model parameters. For example:

$$
F_{r}=f(p)
$$

And

$$
E U R_{30 y r}=f(p)
$$

Where $F_{r}$ is a reservoir or completion parameter being correlated, and $p$ is the time-rate decline model parameter being correlated against.

After establishing individual correlations, we developed multivariate correlating functions for various reservoir/completion parameters and 30 year EUR for each model by establishing a suitable combination of correlating functions. This approach provide relations which can predict reservoir or completion parameters directly from rate decline model parameters.

The integrating parametric correlation functions can be written as:

$$
F_{r}=f\left(p, q, r_{\ldots}\right)
$$

And

$$
E U R_{30 y r}=f\left(p, q, r_{\ldots}\right)
$$

Where $p, q$, and $r$ are the time-rate decline model parameter being correlated against. Finally, we provide cross-plots of calculated and observed reservoir or completion parameters to assess the quality of the parametric correlating functions.

In Fig. 1.5 we show the resulting correlation models for fracture conductivity $\left(F_{c}\right)$ for the various numerical simulation cases. This plot indicates that these functions can provide reasonable estimates of the reservoir/completion properties (in this case, $F_{c}$ and $E U R_{30} y r$ ) for the case of a well within the same reservoir system with similar completion and production constraints. 


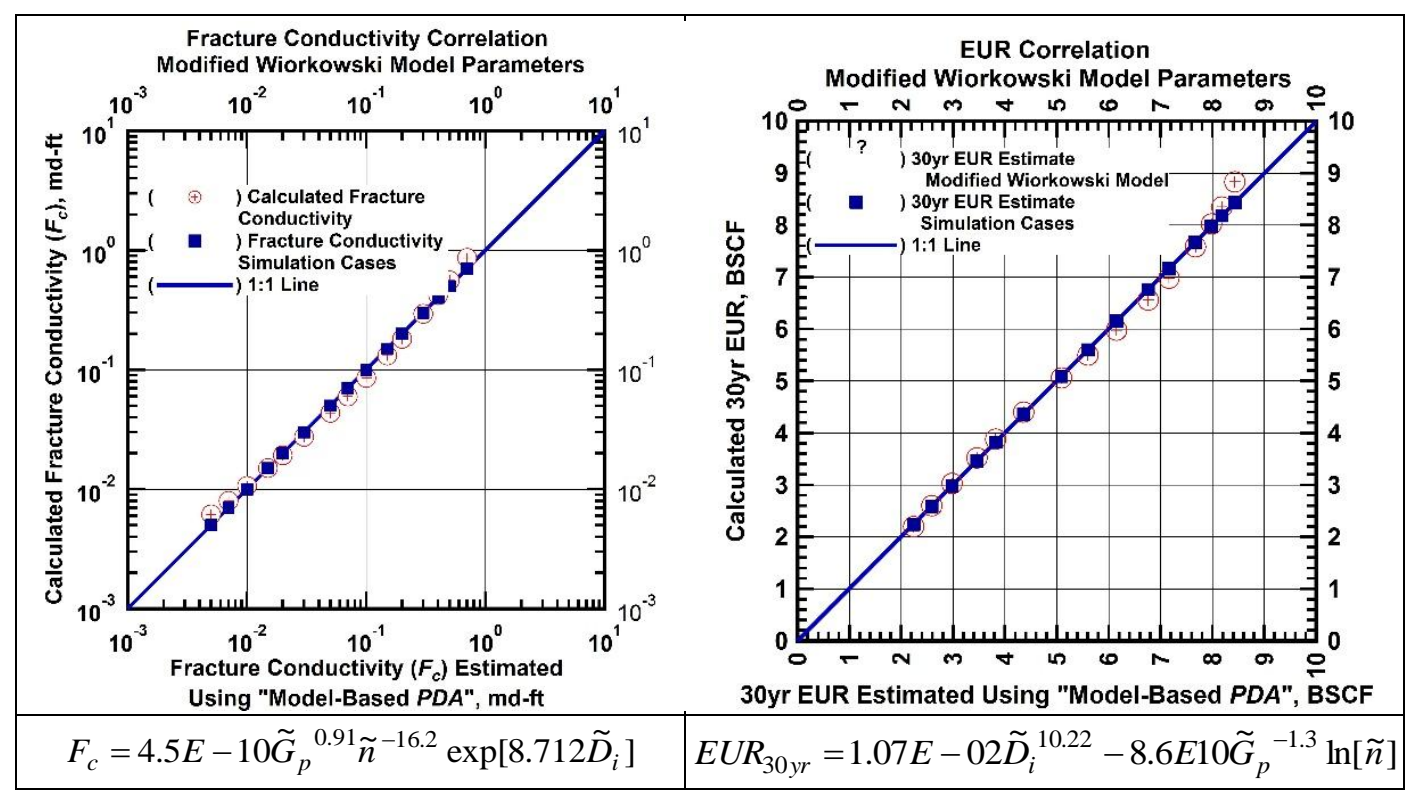

Figure $1.5-$ Comparison of fracture conductivity and 30 year EUR values calculated using parametric correlations developed using the Modified Wiorkowski Model parameters versus results from model-based production analysis (or RTA).

Fig. 1.6 shows the resulting model fits of proposed parametric correlations.

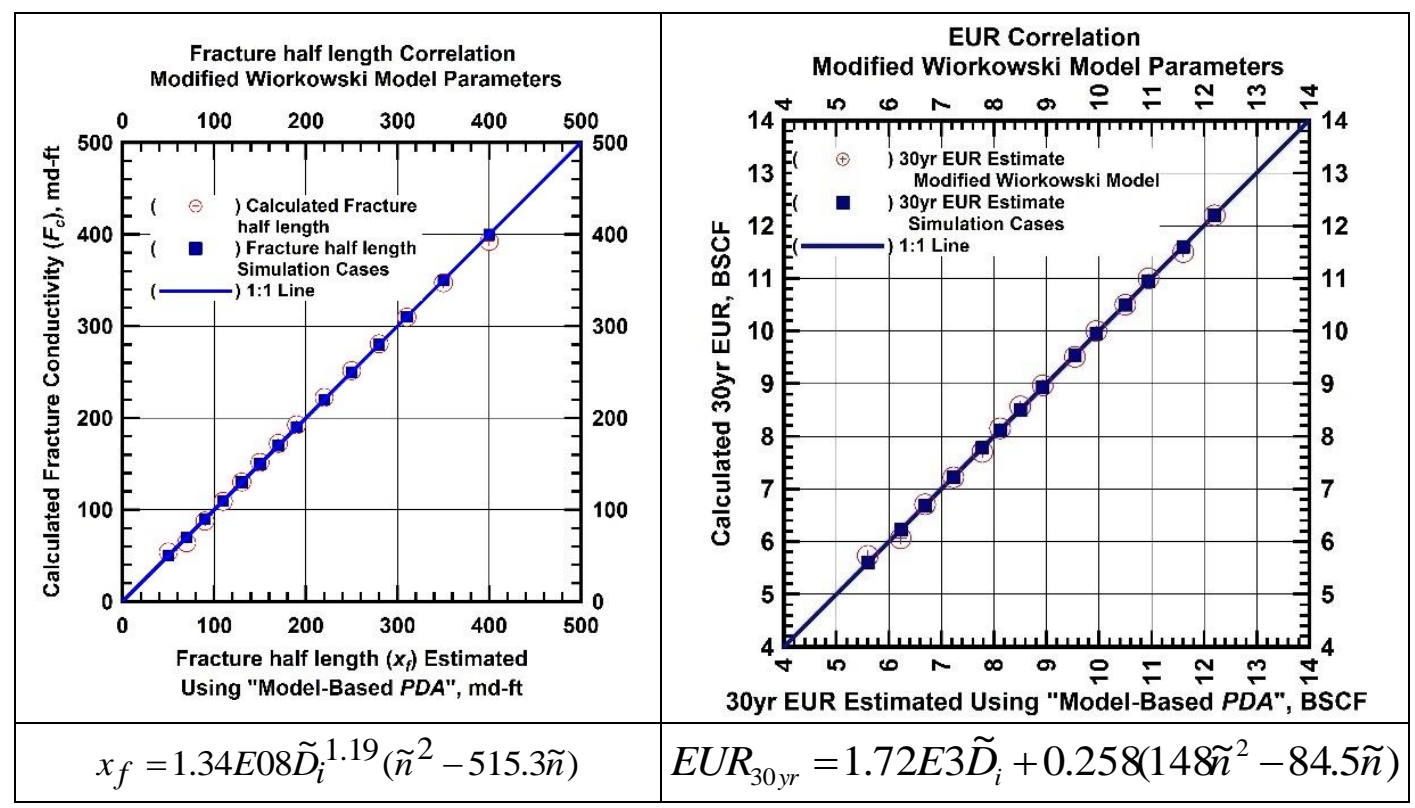

Figure 1.6 - Comparison of fracture half-length and 30 year EUR values calculated using parametric correlations developed using the Modified Wiorkowski Model parameters versus results from model-based production analysis (or RTA). 
Fig. 1.7 shows the resulting model fits of proposed parametric correlations for formation permeability(k). It can be observed that the proposed correlating functions provide a reliable estimate of the reservoir properties.

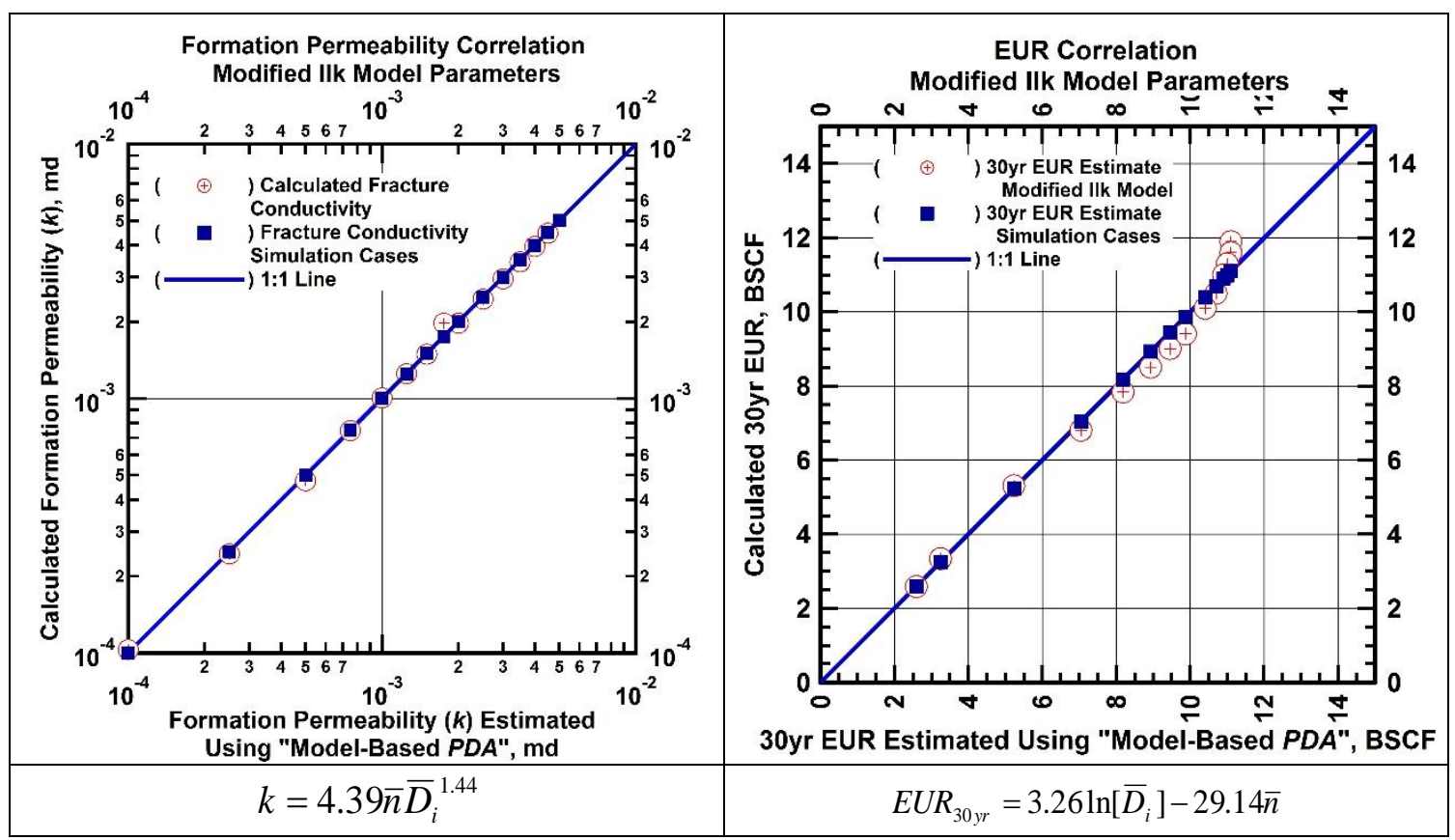

Figure $1.7-$ Comparison of formation permeability and 30 year EUR values calculated using parametric correlations developed using the Modified Wiorkowski Model parameters versus results from model-based production analysis (or RTA). 


\section{CHAPTER II}

\section{LITERATURE REVIEW}

Historical production data analysis techniques have received considerable attention and interest in past few decades because these techniques provide important tools to forecast production and remaining reserves for oil and gas reservoirs, and we can also use these tools to estimate reservoir characteristics. The sophistication of the analysis evolved historically with the changing nature of hydrocarbon assets from conventional to unconventional and with the advancement in technology.

Commonly these techniques can be classified as;

- Classical Time-Rate Analysis

- Modern Time-Rate Analysis

- Semi-analytical and analytical methods

\subsection{Classical Time-Rate Analysis}

Decline curve analysis (or DCA) techniques have been employed by petroleum engineers since the introduction of the Manual for the Oil and Gas Industry under the Revenue Act of 1918 by the US Internal Revenue Service (1919) to estimate oil and gas reserves. Their widespread applicability and acceptance is due to their simplicity and easy formulation. However, historical time-rate relations are empirical in nature and are (strictly) only applicable to boundary-dominated flow conditions. Johnson and Bollens (1927) laid the foundation of traditional time-rate decline curve methods by proposing "loss-ratio" and "loss-ratio derivative" as:

\section{Definition of the Loss-Ratio:}

$$
\frac{1}{D} \equiv-\frac{q}{d q / d t}
$$

Derivative of the Loss-Ratio:

$$
b \equiv \frac{d}{d t}\left[\frac{1}{D}\right] \equiv-\frac{d}{d t}\left[\frac{q}{d q / d t}\right]
$$

Through time, these functions have been designated as the "Arps" relations due to the presentation and use of these relations in the seminal paper by Arps (1945). On a practical note, when the traditional Arps' hyperbolic time-rate and time-cumulative relations are used to forecast production and to estimate reserves, these relations typically overestimate the performance for low to ultra-low permeability reservoirs. 
For reference, the Arps' hyperbolic time-rate and time-cumulative relations are given as:

Arps' Hyperbolic Time-Rate Model:

$$
q(t) \equiv \frac{q_{i, h y p}}{\left(1+b D_{i} t\right)^{1 / b}}
$$

Arps' Hyperbolic Time-Cumulative Model:

$$
G_{p} \equiv \frac{q_{i, h y p}}{(1-b) D_{i}}\left[1-\left(1+b D_{i} t\right)^{1-1 / b}\right]
$$

Arps (1945) used these definitions to derive exponential and hyperbolic relations which are still the most widely used rate-time relations. However, (as mentioned earlier) these relations are only valid (in a "theoretical" sense) for boundary-dominated flow. Arps proposed "derivative of loss-ratio," $b$, as a constant and according to Arps' definition, the $b$-value should vary between 0 and 1 - we note that for $b>1$, unconstrained extrapolations of Eq. 2.4 will tend to infinity, which is obviously not practical (or desired). Therefore, for cases where $b>1$, the extrapolation must be constrained to some limiting time and/or limiting rate.

For the case of wells in low to ultra-low permeability reservoirs, the performance of these wells are characterized by a very long transient flow regime, which often dominates most of the wells productive life (as much as 10-15 years, or more). Often times, the traditional boundary-dominated flow is not observed in production data from wells in ultra-low (unconventional) reservoirs. In such cases, the incorrect application of the Arps' relations (Eqs. 2.3 and 2.4) to performance data for these wells often yields overestimation of reserves.

The issue is that of the "Arps" $b$-parameter (or the "loss-ratio derivative") which is typically on the order of 2 (rather than $b<1-$ i.e., the traditional (boundary-dominated flow) assumption). We note that a $b$ parameter of 2 corresponds (as a coincidence) to the "linear flow" regime which exists for cases where the fractures in an multi-fractured horizontal well (MFHW) have pressure distributions that do not interfere. As is well known in the pressure transient testing literature, the linear flow regime depends on the properties of the hydraulic fractures as well as the permeability of the formation.

In a model-based study, Rushing et al. (2007) observed that the incorrect application of the Arps' relations can result in reserve estimation errors of up to 100 percent. Lee and Sidle (2010) also noted that unconstrained, the hyperbolic time-rate relation can cause reserves to have physically unreasonable properties (i.e., for $b$-parameter values greater than one, the unconstrained reserves extrapolation is infinite (as noted in our comments above)). 
To address the "over-extrapolation" issues related to the hyperbolic time-rate relation, Robertson (1988) proposed a "modified-hyperbolic" time-rate relation. The following variant of the "modified-hyperbolic" relation is the most popular formulation of the "modified-hyperbolic" time-rate relation: (Fekete, 2016)

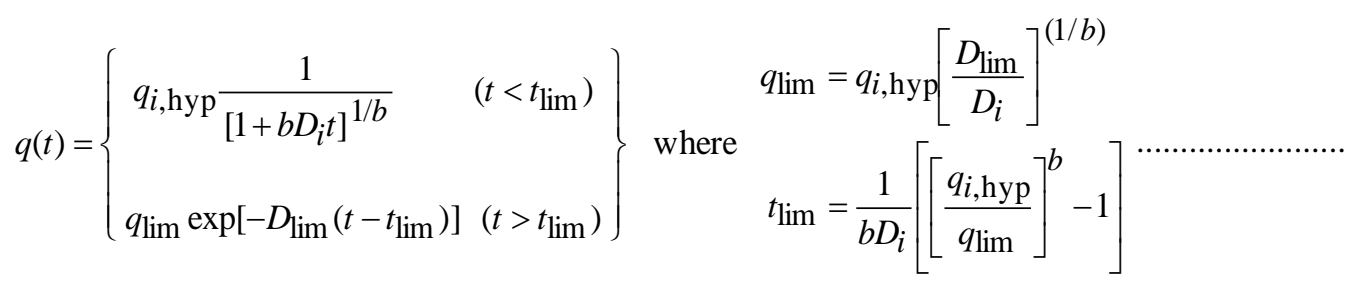

While the "modified-hyperbolic" formulation given by Eq. 2.5 remains the most popular production forecasting/reserves extrapolation tool, this formulation has at least 2 relatively significant issues. First, the selection of the " $D_{\text {lim }}$ " parameter (i.e., the terminal exponential decline rate) is somewhat arbitrary, and it tends to vary between 5-10percent depending on the plan and the company. Second, this formulation tends to provide an average to slightly high forecast/extrapolation, even accounting for the choice of a conservative value of $D_{l i m}$. In addition, the Arps functions ( $D(t)$ and $b(t)$, Eqs. 2.1 and 2.2, respectively) do not often show the constant $b(t)$ behavior required for a "hyperbolic" function, much less the constant $D(t)$ behavior required for an "exponential" function.

Fetkovich (1980) introduced type curves combining analytical solutions from infinite and closed reservoir models with the Arps decline curve relations. Fetkovich provided theoretical basis for Arps' empirical relations by showing that a rate relation with a form identical to Arps' rate decline relations can be obtained by combining material balance relations with pseudosteady-state relations. This observation provides theoretical basis to Arps' empirical relations. This resulted in a matching technique similar to pressure transient analysis which is applicable to both the transient and boundary-dominated flow regimes of the data.

\subsection{Modern Time-Rate Analysis}

Given the nature of the modified-hyperbolic relation not rigorously modeling the performance behavior for unconventional reservoirs, several new models have been proposed by Ilk, et al (2008a, 2008b and 2008c), Valko (2009), Clark, et al(2011), and Mishra (2012). These new models generally result in more realistic matches for wells in low to ultra-low permeability reservoirs which have very long transient and transition flow regimes (Askabe, et al 2012).

Specific to the "diagnostic" behavior for wells in low/ultra-low permeability reservoirs, Ilk, et al. (2008) observed that the $D(t)$ function (computed from data using Eq. 2.1) exhibits a power-law (straight-line) behavior for early-time and transitional flow regimes (hence the name, the "power-law exponential" or 
PLE relation). During late times the PLE model has an imposed "constant decline" that is used to match late-time (i.e., boundary-dominated) flow behavior. As such, the PLE relation can match transient, transition, and boundary-dominated flow behavior. As noted by others, there is often considerable noise evident in field data due to production operations — and, as such, time-rate data sets must be "heavily edited" to yield diagnostic trends from which we can designate the respective flow regimes $(e . g$., transient linear flow, transitional flow, and boundary-dominated flow behavior).

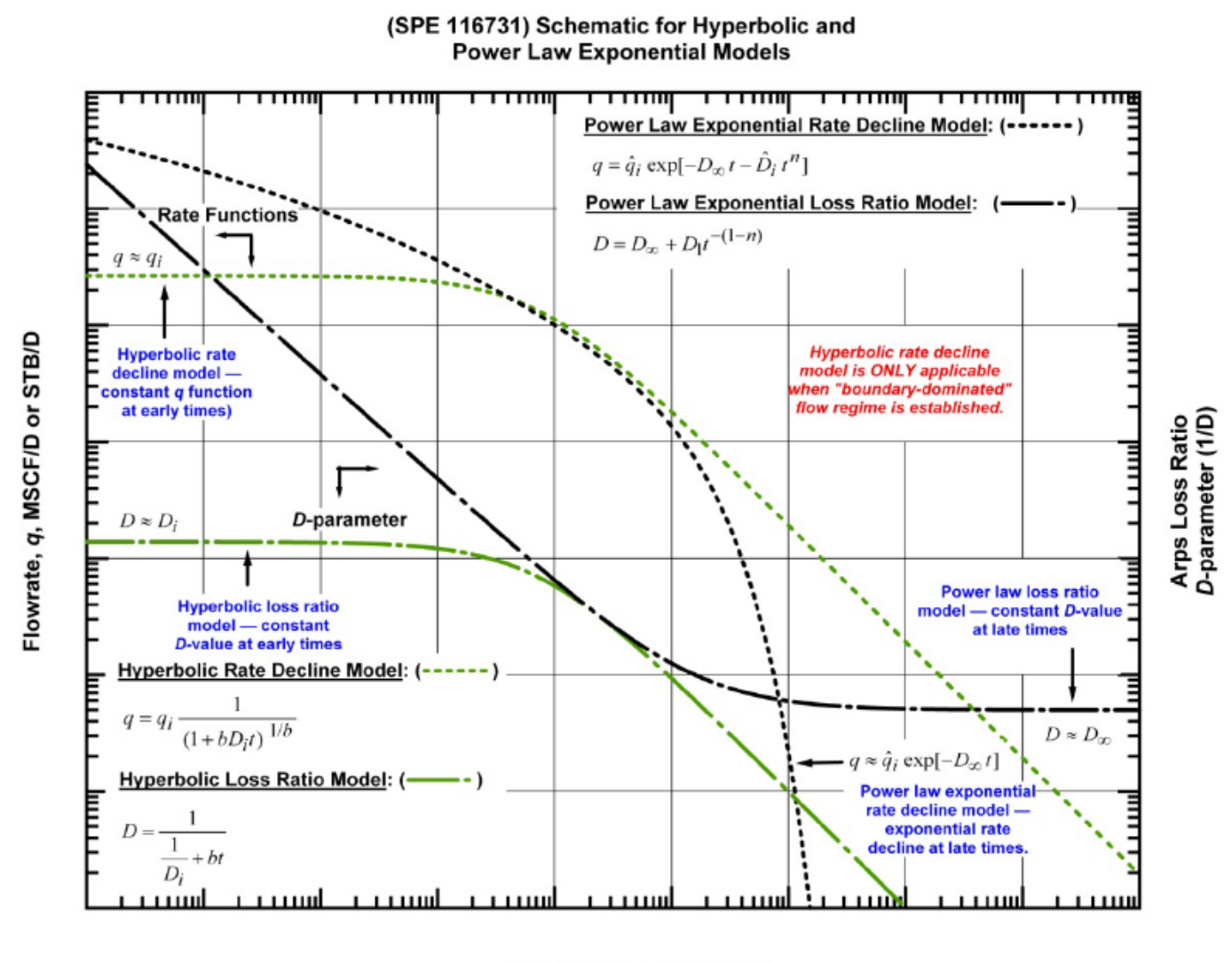

Figure $2.1 \quad$ - (Log-log Plot): Flow rate $\left(q_{g}\right)$ and $D$-parameter versus production time- Hyperbolic and power law exponential rate decline and loss ratio models illustration for orientation purposes (SPE 116731).

Valko (2009) presented the "stretched exponential decline model" (SEDM) while performing a statistical investigation of wells in unconventional reservoirs, where the SEDM was taken from statistical references which represent chaotic and natural processes. In simple terms, the SEDM can be described as a linear superposition of simple exponential decay models with different characteristic times (i.e., an infinite series of exponential terms). This model is identical in form to the empirically-derived power-law exponential 
(PLE) decline model, with the exception that the SEDM does not have a terminal exponential decay term as the PLE does - and as such, the SEDM lacks the (specific) diagnostic behavior necessary to model boundary-dominated flow.

Duong (2011) proposed a time-rate relation based on the observation of a straight line log-log behavior of $q / G_{p}$ (i.e., the inverse material balance time function) versus time for fracture-dominated shale reservoirs (e.g., Fig 2.2). Duong's concept was an attempt to capture the bilinear and linear flow characteristics typically observed in fractured shale wells. However, the Duong model characterizes transient and transition flow regime data only - and does not model late time, boundary-dominated flow behavior. As such, the Duong model tends to overestimate ultimate recovery.

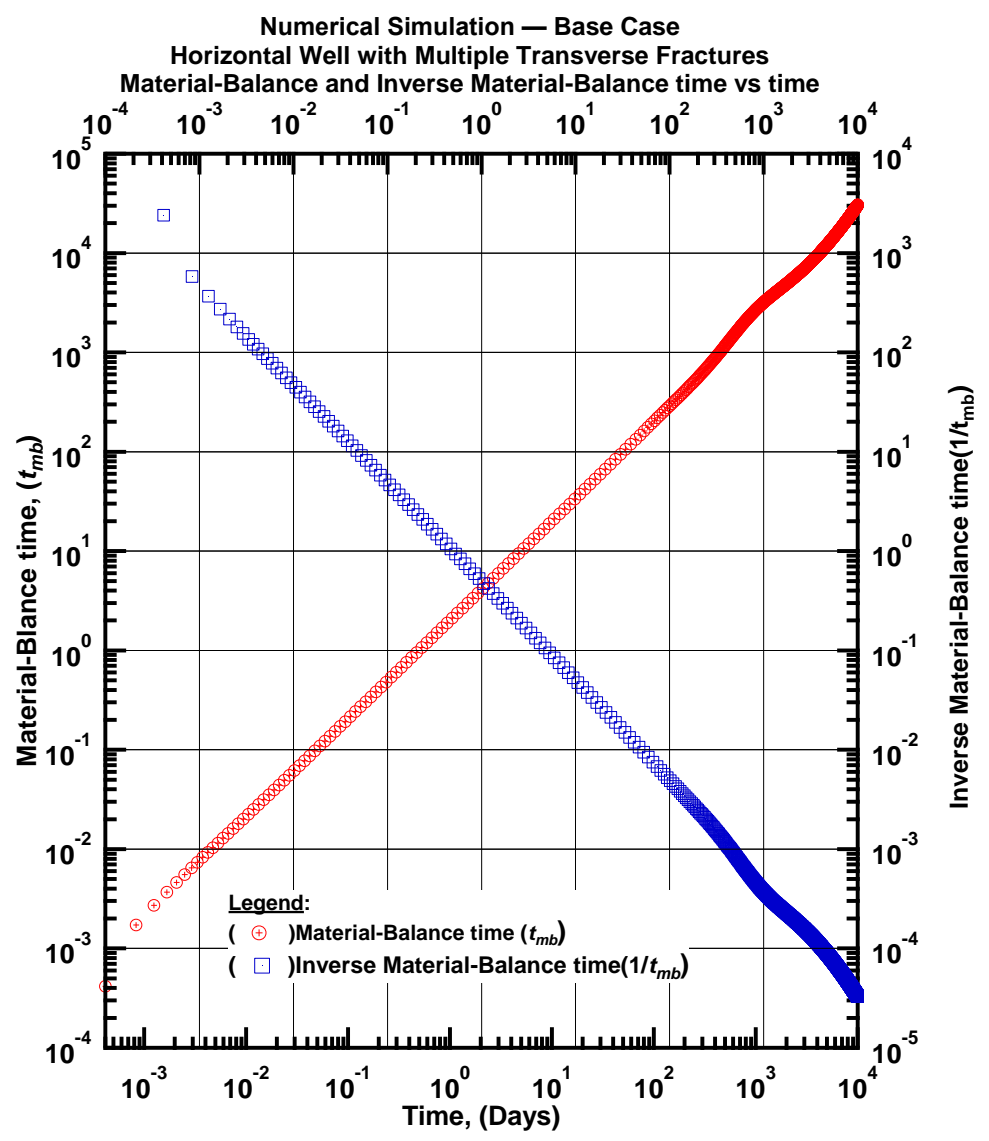

Figure $2.2-$ (Log-log Plot): Material-Balance time characteristic plots for Numerical Simulation Case - material-balance time,(Days) and inverse material-balance time, (1/Days) versus production time,(Days) 
Clark, et al (2011) presented the "logistic growth model" (or LGM) to represent time-rate performance from oil and gas reservoirs. The logistic growth model is taken from standard statistical references and has the unique characteristic that it is constrained by a "carrying-capacity" parameter, where this parameter limits the ultimate growth of the curve. The LGM can match the behavior of long transient and transition flow periods, and to some extent boundary-dominated flow as well (Askabe 2012). In addition, the LGM is similar in behavior to the power-law exponential relation (PLE). As comment, the LGM does not have a specific "diagnostic" behavior where the influence of a given parameter can be observed on a fit-forpurpose diagnostic plot (as does the power-law exponential and Duong models). We note that one of the strengths of the LGM is that the ultimate recovery is a unique parameter in the relation - however, we also note that this feature does not make the LGM more robust as a regression model.

Mishra (2012) presented another self-limited growth model that is based on Weibull growth curves (which are also a family of statistical functions). Analogous to the LGM, the Weibull model constrains cumulative production by use of the "carrying-capacity" parameter. As with the LGM, the Weibull model does not provide specific features for individual parameters (hence, there are no diagnostic plots). Lastly, the Weibull model is also not particularly well-suited as a stand-alone regression model, it is recommended that the Weibull model always be used in conjunction with the LGM and PLE models.

Fulford and Blasingame (2013) proposed a "transient hyperbolic" time-rate model that has as its basis a time-dependent relationship for the Arps' "Loss-Ratio Derivative" Function (i.e., $b(t))$, where $b_{\min }<b(t)$ $<b_{\max }$. This model is implicitly tied to the "end of linear flow" in concept, but in practice this would be very difficult to establish uniquely (i.e., to tie the model parameters to the end of linear flow). This is another "concept model" in an evolving "family" of $b(t)$-based time-rate performance models. The flowrate form of the "transient hyperbolic" model is not written in a compact form, but rather, as an integral based on the defined $b(t)$ and $D(t)$ models. 
Table 2.1 - Summary of Widely Used Time-Rate Relations.

Time-Rate Relations

Arps' Exponential Model

Arps' Hyperbolic Model

Arps' Modified-Hyperbolic Model

Power-Law Exponential Model

Duong Model

Logistic Growth Model
Models

$$
\begin{aligned}
& q(t)=q_{i, \exp } \exp \left(-D_{i} t\right) \\
& q(t)=q_{i, \mathrm{hyp}} \frac{1}{\left[1+b D_{i} t\right]^{1 / b}}
\end{aligned}
$$$$
q(t)=\left\{\begin{array}{ll}
q_{i, \operatorname{hyp}} \frac{1}{\left[1+b D_{i} t\right]^{1 / b}} & \left(t<t_{\lim }\right) \\
q_{\lim } \exp \left[-D_{\lim }\left(t-t_{\lim }\right)\right] & \left(t>t_{\lim }\right)
\end{array}\right\}
$$$$
q(t)=\hat{q}_{i} \exp \left[-D_{\infty} t-\hat{D}_{i} t^{n}\right]
$$$$
q(t)=q_{1} t^{-m} e^{\frac{a}{1-m}\left(t^{1-m}-1\right)}
$$$$
q(t)=\hat{a} K \hat{n} \frac{t^{\hat{n}-1}}{\left(\hat{a}+t^{\hat{n}}\right)^{2}}
$$

Models

$$
\begin{aligned}
& q(t)=\tilde{G}_{p} \tilde{D}_{i} \tilde{a} \tilde{n} \exp \left[-\tilde{D}_{i} t\right]\left(1-\tilde{a} \exp \left[-\tilde{D}_{i} t\right]\right)^{\tilde{n}-1} \\
& q(t)=\bar{G}_{p} \exp \left[-\bar{D}_{\infty} t-\bar{D}_{i} t^{\bar{t}^{n}}\right]\left[\bar{D}_{\infty}+\bar{n} \bar{D}_{i} t^{\bar{n}-1}\right]
\end{aligned}
$$




\section{CHAPTER III}

\section{ANALYSIS OF TIME-RATE RELATIONS}

In this chapter, a detailed analysis of Modified Wiorkowski (MWM) and Modified Ilk model (MIM) is presented. We perform time-rate analysis using the Modified-Wiorkowski Model (MWM) and ModifiedIlk Model (MIM) as a means to forecast gas production from low/ultra-low permeability wells to estimate EUR values. We have used production data generated from a numerical simulation as well as data obtained from a number of field examples including a tight gas well, an Eagle Ford shale oil well, an Eagle Ford shale gas well and Marcellus shale gas well.

We compare the quality of match to specific flow regimes observed from such reservoirs. The data match is conducted by taking full advantage of the characteristics of diagnostic functions including $D-, b$-, parameters, $\beta$-derivative as well as the flow rate data. We used the Bourdet et al.(1989) algorithm to perform the numerical differentiation required to calculate the diagnostic functions. Moreover, we use the "continuous EUR" approach, where EUR is estimated dynamically, to investigate the reliability of the reserve estimates and rate of convergence of $E U R$ when using these models.

\subsection{Modified Wiorkowski Model}

The Wiorkowski cumulative production model (1981) was taken as a variant from the Richards family of "statistical" growth curves and was used to predict ultimate oil production volumes as part of a global oil reserves study. In our work we present a simplified "modified-Wiorkowski" time-cumulative production model. This model and the functions derived from it are presented in detail in Appendix A.

$$
Y(t)=C_{W}\left[1+\gamma_{W} \lambda_{W} C_{W}{ }^{\lambda_{W}} \exp \left[-\alpha_{W} t C_{W}{ }^{\lambda_{W}}\right]\right]^{-\frac{1}{\lambda_{W}}}
$$

Where the coefficients of the Wiorkowski relation are defined as:

$$
\begin{aligned}
& Y=\text { Wiorkowski Model cumulative gas production } \\
& C_{W}=\text { Upper asymptote of Growth Curve } \\
& \gamma_{W}=\text { Model parameter } \\
& \lambda_{W}=\text { Model parameter } \\
& \alpha_{W}=\text { Model parameter }
\end{aligned}
$$

We alternatively proposed a simplified form of Eq. 3.1 as:

$$
G_{p}(t)=\tilde{G}_{p}\left(1-\tilde{a} \exp \left[-\tilde{D}_{i} t\right]\right)^{\tilde{n}}
$$


Where the coefficients of the Wiorkowski relation are defined as:

$$
\begin{array}{ll}
G_{p}(t) & =\text { Cumulative gas production } \\
\tilde{G}_{p} & =\text { EUR upper limit constraint } \\
\tilde{a} & =\text { Model parameter } \\
\tilde{D}_{i} & =\text { Model parameter } \\
\tilde{n} & =\text { Model parameter }
\end{array}
$$

This modification simplifies curve fitting process by eliminating the strong S shaped character of Eq. 3.2 as well as by reducing the complexity of fitting parameters.

From Appendix A, we provide the following subordinate functions derived from Eq. 3.2:

Time-Rate Relation: (ref. Eq. A-2)

$$
q(t)=\tilde{G}_{p} \tilde{D}_{i} \tilde{a} \tilde{n} \exp \left[-\tilde{D}_{i} t\right]\left(1-\tilde{a} \exp \left[-\tilde{D}_{i} t\right]\right)^{\tilde{n}-1}
$$

Rate-Cumulative Relation:(ref. Eq. A-9)

$$
q(t)=\tilde{D}_{i} \tilde{n} \frac{1}{\tilde{a}}\left[\left[\frac{G_{p}(t)}{\widetilde{G}_{p}}\right]-\frac{1}{\tilde{n}}-1\right] G_{p}(t)
$$

$\underline{D(t) \text { Function:(ref. Eq. A-13) }}$

$$
D(t)=\frac{\tilde{D}_{i}\left(-\tilde{a} \tilde{n}+\exp \left[\tilde{D}_{i} t\right]\right)}{-\tilde{a}+\exp \left[\tilde{D}_{i} t\right]}
$$

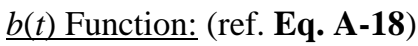

$$
b(t)=\frac{\tilde{a}(\tilde{n}-1) \exp \left[\tilde{D}_{i} t\right]}{\left[-\tilde{a} \tilde{n}+\exp \left[\tilde{D}_{i} t\right]\right]^{2}}
$$

$\beta(t)$ Function: (ref. Eq. A-21)

$$
\beta(t)=\frac{\tilde{D}_{i} t\left(-\tilde{a} \tilde{n}+\exp \left[\tilde{D}_{i} t\right]\right)}{-\tilde{a}+\exp \left[\tilde{D}_{i} t\right]}
$$

It can be seen from Eqs. 3.4 and 3.5 that there is no clear "diagnostic" trend for the $D(t)$ and $b(t)$ functions for the modified-Wiorkowski model (MWM) - i.e., it is not obvious that a plot of $D(t)$ or $b(t)$ versus $t$ (in any particular format) will yield a "diagnostic" trend where the coefficients in these relations can be determined directly from graphical analysis methods. This is not necessarily a limitation of the modifiedWiorkowski model, but these characteristics limit our analysis to regression methods. As will be seen in 
later sections, the MWM model provides excellent matches to the transient, transition and boundarydominated flow regimes.

\subsection{Modified Ilk Model}

This model is inspired from the power law exponential rate-time relation proposed by Ilk et al. (2008). Ilk et al. (2008) observed that data from the transient and transition flow regimes in unconventional low/ultralow permeability reservoirs are characterized by a power-law relation or a straight line on a log-log plot of "inverse loss-ratio", $D$-parameter, versus time. To accommodate the late-time boundary-dominated flow data, the Modified Ilk Model (MIM) uses a constant exponential decline at late times, thus providing a constraint on reserves. This constraint is represented by a constant decline parameter, $D_{\infty}$, which provides constant exponential decline at late time in the life of the well. This parameter is approximated by parameter $\bar{D}_{\infty}$ in our cumulative-time relation.

Recalling the "Ilk et al" time-rate model (2008), we have:

$$
q(t)=\hat{q}_{i} \exp \left[-D_{\infty} t-D_{i} t^{n}\right]
$$

Conceptually, we believe that the form of the Modified-Ilk time-cumulative model (MIM) can be written intuitively from Eq. 3.7 as:

$$
G_{p}(t)=\bar{G}_{p}\left(1-\exp \left[-\bar{D}_{\infty} t-\bar{D}_{i} t^{\bar{n}}\right]\right)
$$

Where the coefficients of the Wiorkowski relation are defined as:

$$
\begin{array}{ll}
G_{p}(t) & =\text { Cumulative gas production } \\
\bar{G}_{p} & =\text { EUR upper limit constraint } \\
\bar{D}_{\infty} & =\text { Model parameter } \\
\bar{D}_{i} & =\text { Model parameter } \\
\bar{n} & =\text { Model parameter }
\end{array}
$$

Where we note that Eq. 3.8 is NOT the actual integral of Eq. 3.7, but rather, this is an intuitive form taken from the Ilk time-rate model and written in a time-cumulative formulation. In Appendix B, we provide the following subordinate functions derived from Eq. 3.8:

Time-Rate Relation: (ref. Eq. B-3)

$$
q(t)=\bar{G}_{p} \exp \left[-\bar{D}_{\infty} t-\bar{D}_{i} t^{\bar{n}}\right]\left(\bar{D}_{\infty}+\bar{n} \bar{D}_{i} t^{\bar{n}-1}\right)
$$

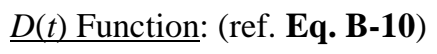

$$
D(t)=\bar{D}_{\infty}+\frac{1-\bar{n}+\overline{D_{i}} \bar{n} t^{\bar{n}}}{t}+\frac{(\bar{n}-1) \bar{D}_{\infty}}{\left(\bar{D}_{\infty} t+\bar{n} \bar{D}_{i} t^{\bar{n}}\right)}
$$




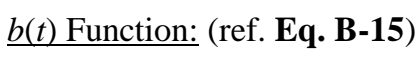

$$
b(t)=-\frac{\bar{D}_{i}(\bar{n}-1) c t^{c}\left[\bar{D}_{i}{ }^{2} \bar{n}^{2} t^{2 \bar{n}}+\bar{D}_{\infty} t\left(2-\bar{n}+\bar{D}_{\infty} t\right)+\bar{D}_{i} \bar{n} t^{\bar{n}}\left(1+2 \bar{D}_{\infty} t\right)\right]}{\left[\bar{D}_{\infty}{ }^{2} t^{2}+2 \bar{D}_{\infty} \bar{D}_{i} \bar{n} t^{1-\bar{n}}+\bar{D}_{i} \bar{n} t^{\bar{n}}\left[1+\bar{n}\left(\bar{D}_{i} t^{\bar{n}}-1\right)\right]\right]^{2}}
$$

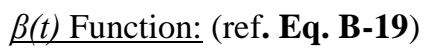

$$
\beta(t)=\bar{D}_{\infty} t+(1-\bar{n})+\bar{D}_{i} \bar{n} t^{\bar{n}}+\frac{(\bar{n}-1) \bar{D}_{\infty} t}{\left(\bar{D}_{\infty} t+\bar{n} \bar{D}_{i} t^{\bar{n}}\right)}
$$

For the "Modified-Ilk" $D(t)$ relation (Eq. 3.10) we note something akin to a power-law trend (i.e., a possible straight line trend of $D(t)$ versus $t$ on a log-log plot), although it is not obvious how the 2nd and 3rd terms in this relation will interact. We will test this and all functions using simulated and actual reservoir performance later in this work. As for the $b(t)$ function proposed by Eq. 3.11, this behavior appears to be quite complicated, but it is possible that this trend may also be "nearly" power-law (i.e., $b(t)$ versus $t$ forms an approximate straight-line trend on a log-log plot).

As will be seen in later sections, the Modified Ilk Model (MIM) provides an excellent match to the transient, transition and boundary-dominated flow regimes and provides means to self-constraint unlimited growth of reserves that might, otherwise, would have been the case.

\subsection{Time-Cumulative Diagnostics}

A novel aspect of this work is the development of the so-called "time-cumulative diagnostics" which are based on cumulative production rather than flow rate (analogous to the Arps $D(t)$ and $b(t)$ diagnostic functions). These functions are proposed as:

"Cumulative Loss-Ratio" Function:

$$
\frac{1}{D_{c}(t)}=\frac{G_{p}(t)}{d G_{p}(t) / d t}=\frac{G_{p}(t)}{q(t)}=t_{m b}
$$

"Inverse Cumulative Loss-Ratio" Function:

$$
D_{c}(t)=\frac{1}{G_{p}(t)} \frac{d G_{p}(t)}{d t}=\frac{1}{G_{p}(t)} q(t)=\frac{1}{t_{m b}}
$$

"Derivative of the Cumulative Loss-Ratio" Function:

$$
b_{c}(t)=\frac{d}{d t}\left[\frac{1}{D_{c}(t)}\right]=\frac{d}{d t}\left[\frac{G_{p}(t)}{d D_{c}(t) / d t}\right]=\frac{d}{d t}\left[t_{m b}\right]
$$

Where $t_{m b}$ is the "material balance" time and is defined as:

$$
t_{m b}=\frac{G_{p}(t)}{q(t)}
$$




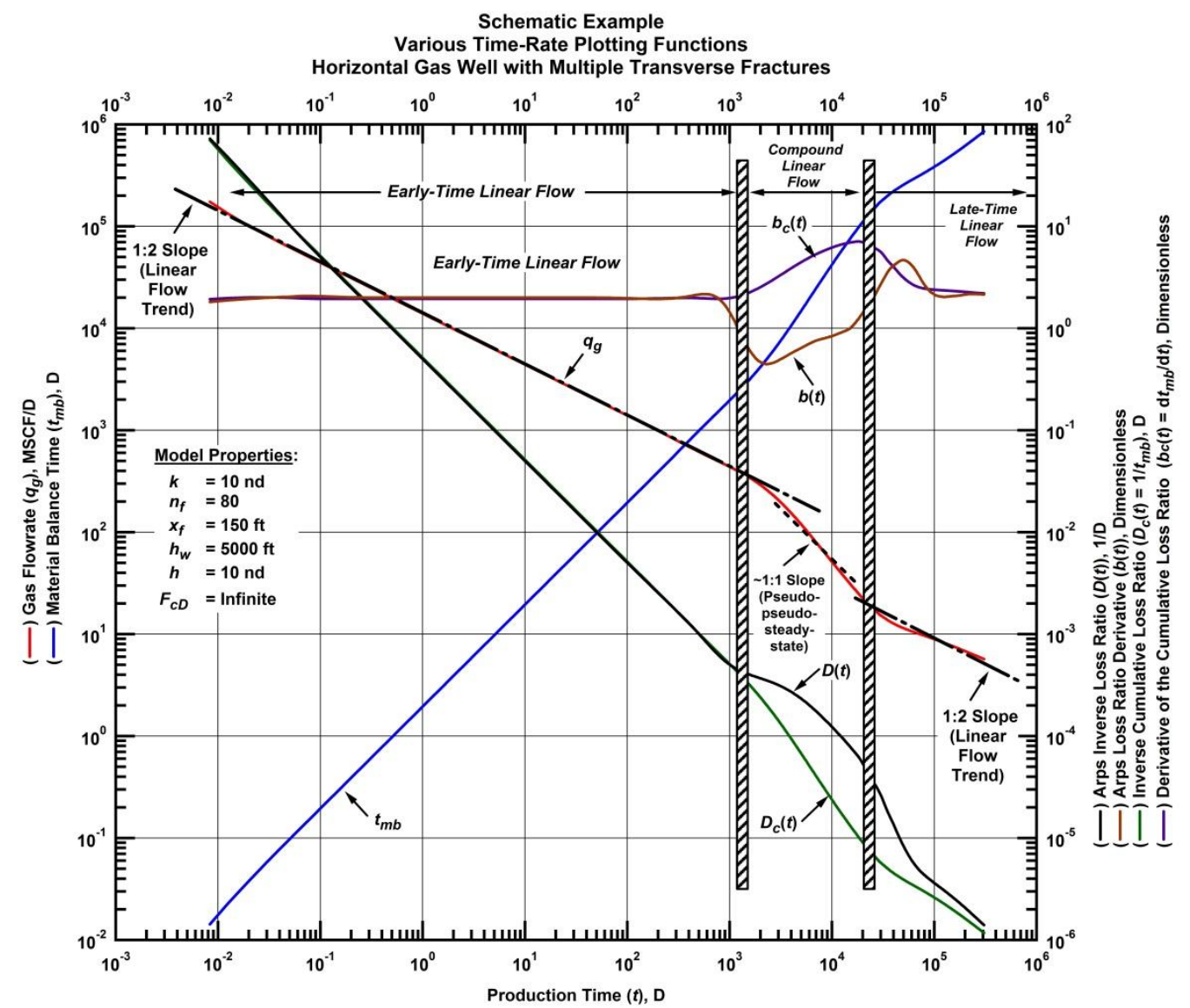

Figure 3.1 - (Log-log Plot) Schematic example of time-rate plotting functions for a horizontal gas well with multiple transverse fractures (numerical simulation case).

We immediately note that the $D_{c}(t)$ and $b_{c}(t)$ functions can be written solely as a function of the material balance time function $\left(t_{m b}\right)$ whose application in decline curve analysis are discussed by Doublet et al.(1994). While not a specific objective of this work, we note that these functions are uniquely defined by the material balance time function, which may lead to simplified diagnostic plots and interpretations. As noted earlier, Duong (2011) proposed a time-rate relation based on the empirical observation of a straightline log-log behavior of $q / G_{p}$ (or $\left.1 / t_{m b}\right)$ versus time for "fracture dominated shale reservoirs". While it is not our objective to "tie" these "time-cumulative diagnostic" functions to the Duong methodology, there may be some relevance in that effort and we encourage others to consider such work.

\subsection{Validation - Synthetic \& Field Examples}

In this section, we present validation of Modified Wiorkowski (MWM) and Modified Ilk (MIM) models using both synthetic and field data. We use the $q_{g}(t), D(t)$ and $b(t)$ functions derived from production data 
(i.e., the " $q D b "$ plot). We also provide a "continuous EUR" analysis (Currie et al. 2010) using the MWM and MIM models to establish the time-dependent nature of the EUR behavior.

\subsubsection{Field Example: Numerical Simulation (Synthetic) Case}

For this numerical simulation case, we consider a low permeability horizontal well with multiple transverse fractures and we have generated a production profile for 10,000 days ( $~ 30$ years) as a means of validating the $E U R_{30 Y r}$ (estimated ultimate recovery at 30 years). The well is produced at a constant bottomhole pressure of 1,000 psia. The model input parameters are provided in Table 3.1. The historical flow rate and cumulative production data is shown in Fig. 3.2. All numerical simulation production profiles in this work are generated using Ecrin from Kappa Engineering Softwares.

Table 3.1 - Reservoir and fluid properties for numerical simulation case (horizontal well with multiple transverse fractures).

$\begin{array}{lll}\text { Reservoir Properties } & & \\ \text { Net pay thickness, } h & = & 200 \mathrm{ft} \\ \text { Formation permeability, } k & = & 1 \times 10^{-4} \mathrm{md} \\ \text { Fracture conductivity, } F_{c D} & = & \text { infinite } \\ \text { Wellbore Radius, } r_{w} & = & 0.25 \mathrm{ft} \\ \text { Formation compressibility, } c_{f}= & 3 \times 10^{-6} \mathrm{psia}^{-1} \\ \text { Porosity, } \phi & = & 0.05(\mathrm{fraction}) \\ \text { Initial reservoir pressure, } p_{i} & = & 5,000 \mathrm{psia} \\ \quad \text { Gas saturation, } s_{g} & = & 0.65 \text { fraction } \\ \quad \text { Skin factor, } s & = & 0(\text { dimensionless }) \\ \text { Reservoir temperature, } T_{r} & = & 212^{\circ} \mathrm{F} \\ \text { Fluid properties: } & & \\ \text { Gas specific gravity, } \gamma_{g} & = & 0.6(\text { air }=1) \\ \text { Hydraulically fractured well model parameters: } & \\ \text { Fracture half-length, } x_{f} & = & 200 \mathrm{ft} \\ \text { Number of fractures } & = & 100 \\ \text { Horizontal well length, } L_{h} & = & 5,000 \mathrm{ft} \\ \text { Production parameters: } & & \\ \text { Last tubing pressure, } p_{w f} & = & 1,000 \mathrm{psia} \\ \text { Production time, } t & = & 10,000 \text { days }(\sim 30 \text { years })\end{array}$




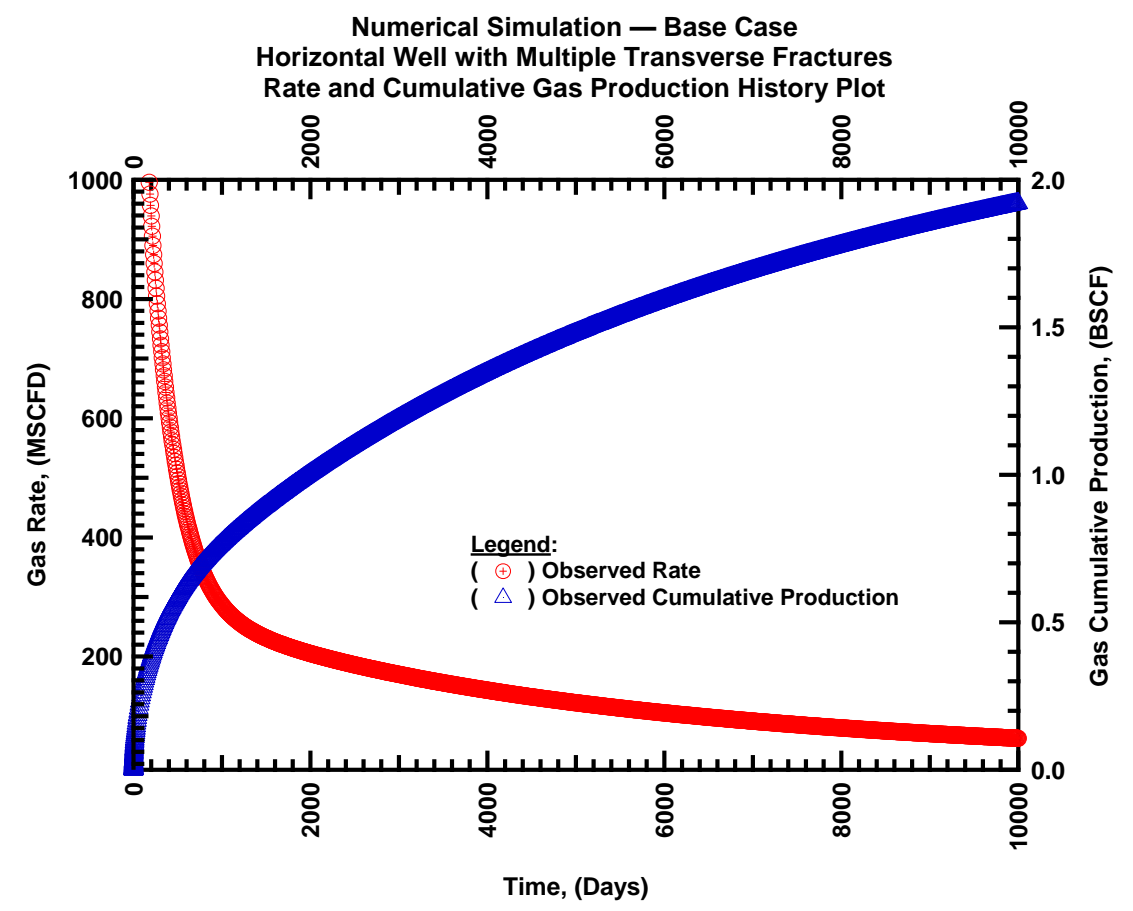

Figure 3.2 - (Cartesian Plot): Production history plot for numerical simulation case - flow rate,(MSCFD) and cumulative production, (MSCF) versus production time,(Days)

A summary of time-rate relations of all models used for this comparative study are presented in Table.

3.2. The table also shows the newly derived time-rate relations.

Table 3.2 - PLE, Duong, logistic growth model and newly derived time-rate relations.

\begin{tabular}{|c|c|}
\hline Time-Rate Relations & Models \\
\hline Power-Law Exponential Model & $q(t)=\hat{q}_{g i} \exp \left[-D_{\infty} t-\hat{D}_{i} t^{n}\right]$ \\
\hline Duong Model & $q(t)=q_{1} t^{-m} \exp \left[\frac{a}{1-m}\left(t^{1-m}-1\right)\right], q_{1}$ at $t=1$ \\
\hline Logistic Growth Model & $q(t)=\frac{\hat{a} K \hat{n} t^{\hat{n}-1}}{\left(\hat{a}+t^{\hat{n}}\right)^{2}}$ \\
\hline Arps' Hyperbolic Model & $q(t)=\frac{\hat{q}_{g i}}{\left(1+\sum_{i} b t\right)^{1 / b}}$ \\
\hline Arps' Exponential Model & $q(t)=\hat{q}_{g i} \exp \left[-D_{i} t\right]$ \\
\hline New Time-Rate Relations & Models \\
\hline Modified Wiorkowski Model & $q(t)=\tilde{G}_{p} \tilde{D}_{i} \tilde{a} \tilde{n} \exp \left[-\tilde{D}_{i} t\right]\left(1-\tilde{a} \exp \left[-\tilde{D}_{i} t\right]\right)^{\tilde{n}-1}$ \\
\hline Modified Ilk Model & $q(t)=\bar{G}_{p} \exp \left[-\bar{D}_{\infty} t-\bar{D}_{i} t^{\bar{n}}\right]\left(\bar{D}_{\infty}+\bar{n} \bar{D}_{i} t^{\bar{n}-1}\right)$ \\
\hline
\end{tabular}


In Fig. 3.3, we present the " $q D b$-plot" plot for this case. On the $q D b$-plot the following functions are plotted -Flowrate $\left[q_{g}(t)\right]$, Arps' Inverse Loss Ratio $[D(t)]$, and Arps' Loss Ratio Derivative $[b(t)]$ versus production time. In this case we plot the $D(t)$ and $b(t)$ data functions (symbols) as well as the $D(t)$ and $b(t)$ model functions (lines) for the Power-Law Exponential (PLE) model, the Logistic Growth Model (LGM), the Duong model, the Modified-Wiorkowski model (MWM), and the Modified-Ilk model (MIM).

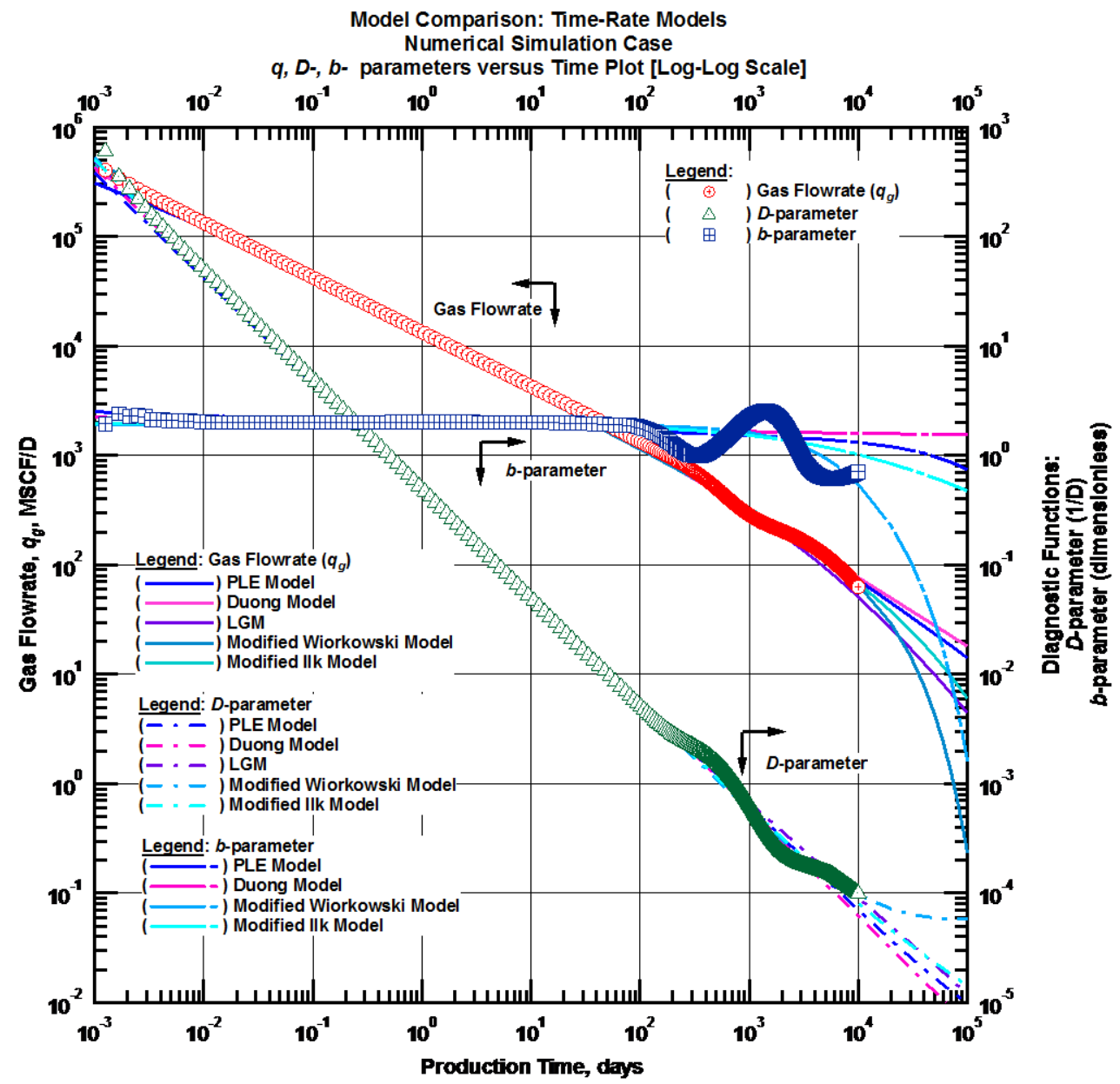

Figure 3.3 - (Log-log Plot): Flow rate $\left(q_{g}\right), D$ - and $b$-parameter versus production time. PLE, Logistic growth, Duong, MWM and MIM time-rate model matches for numerical simulation case.

It can be observed that all models give reasonable rate and cumulative matches. Moreover, the Modified Wiorkowski Model(MWM) along with the Modified Ilk Model(MIM) yield the most conservative estimates of 30-year EUR for the numerical simulation case. This behavior can be attributed to the selflimiting growth nature of both models which results in inherently conservative matches across transient, 
transition and boundary-dominated flow regimes for low/ultra-low permeability wells. The $D(t)$ and $b(t)$ data functions do capture the (apparent) end of linear flow observed in the flow rate data about 700 days, but the later flow transition flow and apparent boundary-dominated flow regimes are not so clearly defined. In fact, focusing solely on the $D(t)$ data function, we note that this function suggests essentially only a single trend (i.e., all of the data shown by the green symbols could, in a practical sense, be captured by a single power-law, straight-line trend).

Table 3.3 provides a summary of the results for all of the time-rate decline model applied to the example (fully) numerical simulation case. We note from Fig. 3.4 that all of the matches are reasonable. However the Modified-Wiorkowski Model (MWM) and the Modified-Ilk Model (MIM) model yield the most conservative estimates of 30-year EUR (i.e., EUR30Yr). We believe that this "conservative" behavior is attributed to the self-limiting growth capability of both the MWM and MIM models.

Table 3.3 - Summary of Decline Curve Analyses $(E U R)$ for the Numerical Simulation Case. $\left(G_{p, \max }=1.92 \mathrm{BSCF}\right.$ at 30 years from numerical simulation. $)$

\begin{tabular}{lll} 
Time-rate models & & $G_{p, \max }$ \\
\cline { 1 - 1 } Duong model & & $2.70 \mathrm{BSCF}$ \\
Logistic growth model & & $2.49 \mathrm{BSCF}$ \\
Power-law exponential model (PLE) & & $2.74 \mathrm{BSCF}$ \\
Arps' Hyperbolic Model & & $2.89 \mathrm{BSCF}$ \\
Modified Wiorkowski Model & & $1.79 \mathrm{BSCF}$ \\
Modified Ilk Model & & $1.93 \mathrm{BSCF}$
\end{tabular}

Fig. 3.4 shows the "Continuous EUR" results versus production time for the MWM and MIM. For the numerical simulation case we observe convergence to the 30-year EUR (actually the gas produced at 30 years since this is a simulation case) at around 3,000 days (or approximately 10 years), which is consistent with expectations for a multi-fracture horizontal well (MFHW) producing in an ultra-low permeability formation. 


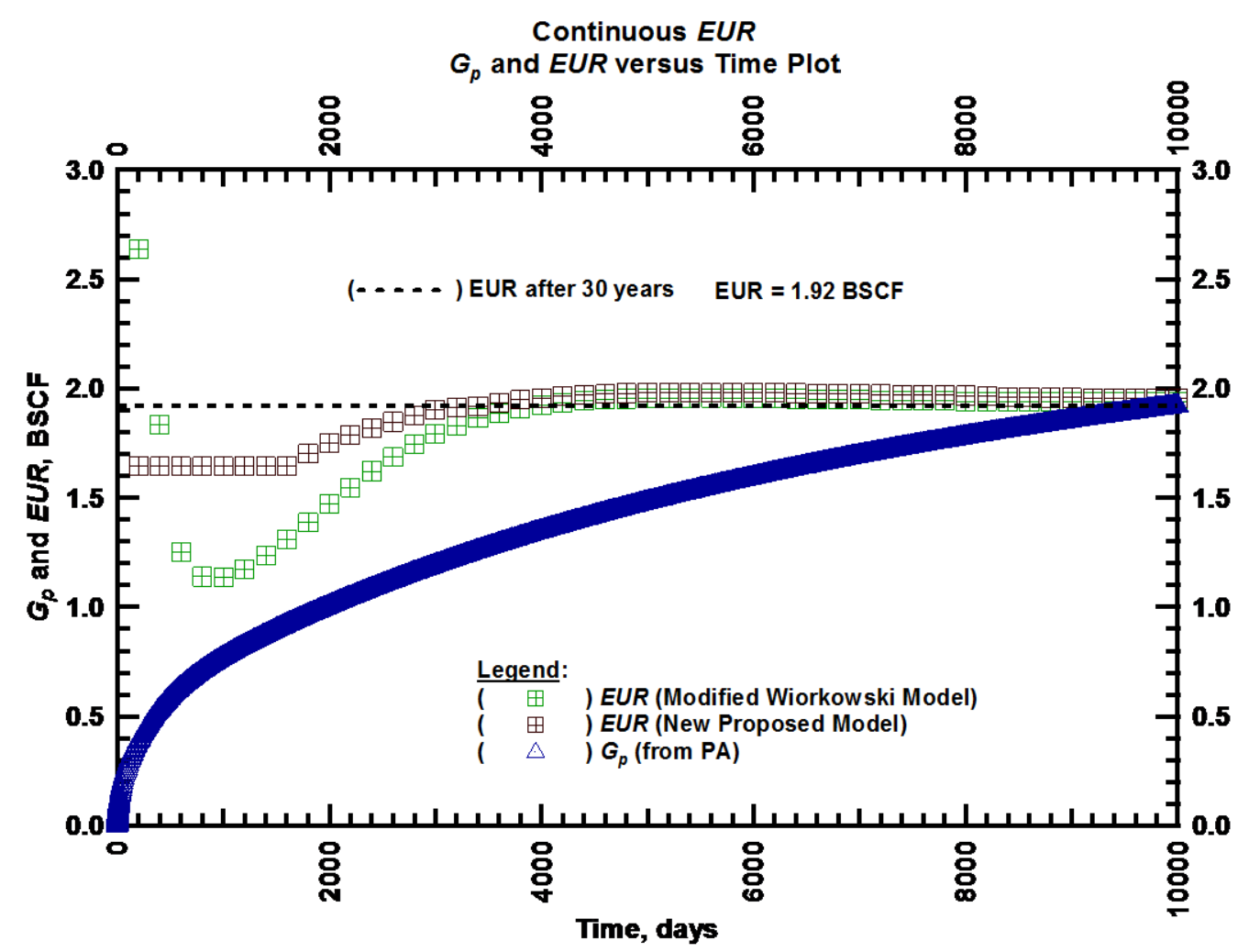

Figure 3.4- (Cartesian Plot): EUR estimates from Modified Wiorkowski and Modified Ilk Models and $\mathrm{G}_{p, \max }$ projected from numerical simulation case.

\subsubsection{Field Example: East Texas Gas Well Case}

The "East Texas Gas Well" case is taken from the literature and considers the case of a vertical well with a single vertical fracture of finite fracture conductivity in a "tight gas" formation of approximately $0.005 \mathrm{md}$. This particular case has about 5000 days of production performance data available and boundarydominated flow is well-established.

Table 3.4 shows reservoir and well parameters used to generate production data for this numerical simulation case. 
Table 3.4 - Reservoir and fluid properties for East Texas Tight Gas Well Reservoir Properties

Net pay thickness, $h \quad=\quad 177 \mathrm{ft}$

Formation permeability, $k=6 \mu \mathrm{D}$

Wellbore Radius, $r_{w}=0.33 \mathrm{ft}$

Formation compressibility, $c_{f}=1 \times 10^{-7} \mathrm{psi}^{-1}$

Porosity, $\phi \quad=0.088$ (fraction)

Initial reservoir pressure, $p_{i}=9330 \mathrm{psi}$

Gas saturation, $s_{g} \quad=\quad 0.87$ fraction

Skin factor, $s \quad=0.14$ (dimensionless)

Reservoir temperature, $T_{r}=300^{\circ} \mathrm{F}$

Fluid properties:

Gas specific gravity, $\gamma_{g} \quad=\quad 0.7($ air $=1)$

Hydraulically fractured well model parameters:

Fracture half-length, $x_{f} \quad=\quad 365 \mathrm{ft}$

Production parameters:

Production time, $t \quad=\quad 5,216$ days

We begin our diagnostic analysis by removing data points that deviate from the dominant underlying production trend - this action is critical because of the influence of the erratic production data the timerate analysis. In Fig. 3.5 we consider the flow rate function where the "edited" points are shown by the red symbols and the "deleted" points are shown by the light gray symbols. Once the editing process is completed, the $D(t)$ and $b(t)$ functions are computed using the so-called "Bourdet" algorithm used for computing the derivative functions in pressure transient analysis.

Typically tight gas wells have very low permeability (on the scale of micro-darcies) and the production history is often very erratic with many large fluctuations especially at early-times. Fig. 3.5 gives a semi$\log$ production history plot of East Texas Tight Gas Well. 


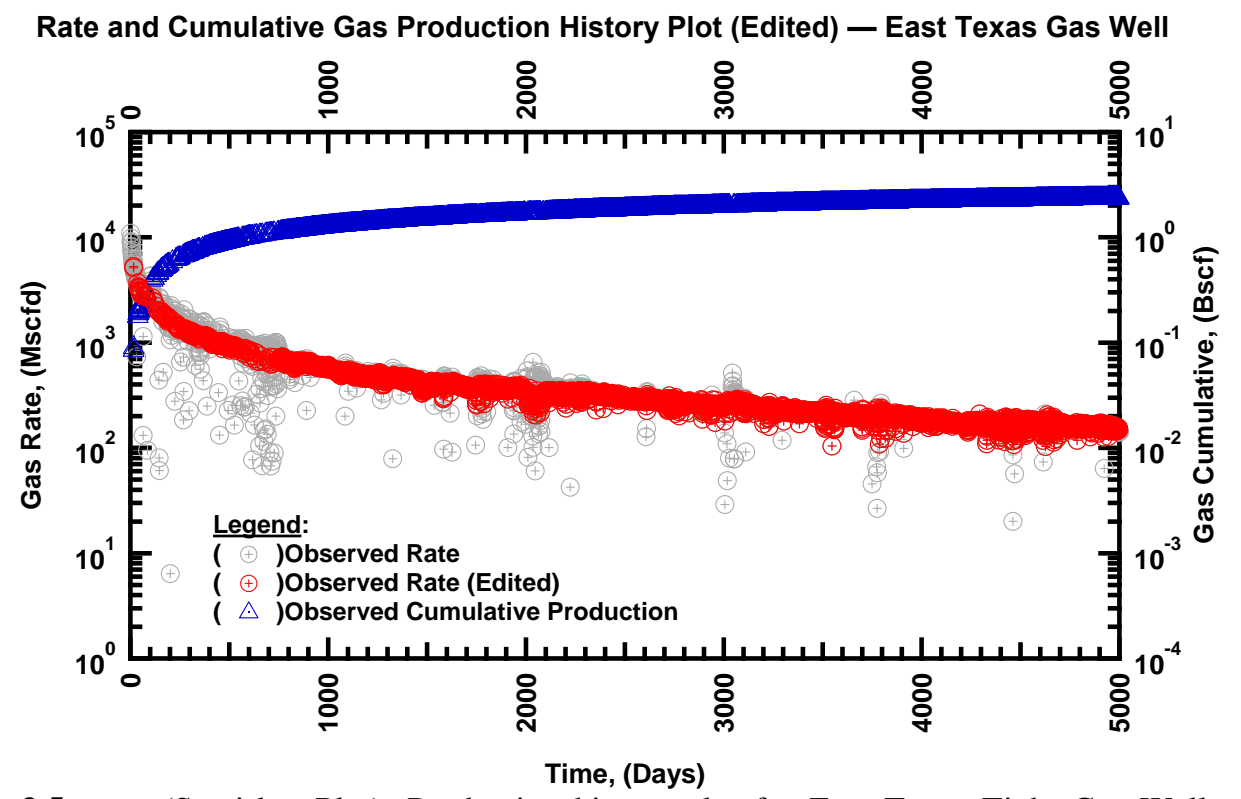

Figure 3.5 - (Semi-log Plot): Production history plot for East Texas Tight Gas Well - flow rate,(MSCFD) and cumulative production, (BSCF) versus production time,(Days)

The Modified Wiorkowski and Modified Ilk Models were matched for 5,000 days of available production history of East Texas Tight Gas Well to obtain 30-year EUR forecasts. In Table 3.5 we provide the match parameters for both models.

Table 3.5 - Modified Wiorkowski and Modified Ilk Models matched parameters for East Texas Tight Gas Well

\begin{tabular}{|c|c|c|c|}
\hline \multicolumn{4}{|c|}{ Modified Wiorkowski Model } \\
\hline $\begin{array}{c}\tilde{G}_{p} \\
(\mathrm{MSCF})\end{array}$ & $\tilde{a}$ & $\tilde{n}$ & $\tilde{D}_{i}\left(\mathrm{D}^{-1}\right)$ \\
\hline $3.4 \times 10^{6}$ & 1 & 0.42 & 0.000141 \\
\hline \multicolumn{4}{|c|}{ Modified Ilk Model } \\
\hline$\overline{G_{p}}$ & & & \\
\hline (MSCF) & $\bar{D}_{\infty}$ & $\bar{n}$ & $\bar{D}_{i}\left(\mathrm{D}^{-1}\right)$ \\
\hline $3.9 \times 10^{6}$ & $1 \times 10^{-7}$ & 0.53 & 0.01102 \\
\hline
\end{tabular}

In Fig. 3.6 we only utilize the Modified-Wiorkowski Model (MWM) and Modified-Ilk Model (MIM) time-rate models for clarity. As the East Texas Gas Well has a production history of considerable duration, we can expect very strong diagnostic trends (i.e., the $D(t)$ and $b(t)$ functions). In particular, we 
note an almost perfect straight-line trend in the $D(t)$ function, which is the defining characteristic of the Power-Law Exponential time-rate model. We note excellent matches using the MWM and MIM models, and we can suggest, based on the extrapolations of these models, that their "self-constraining" characteristics provide average to conservative performance predictions and EUR estimates.

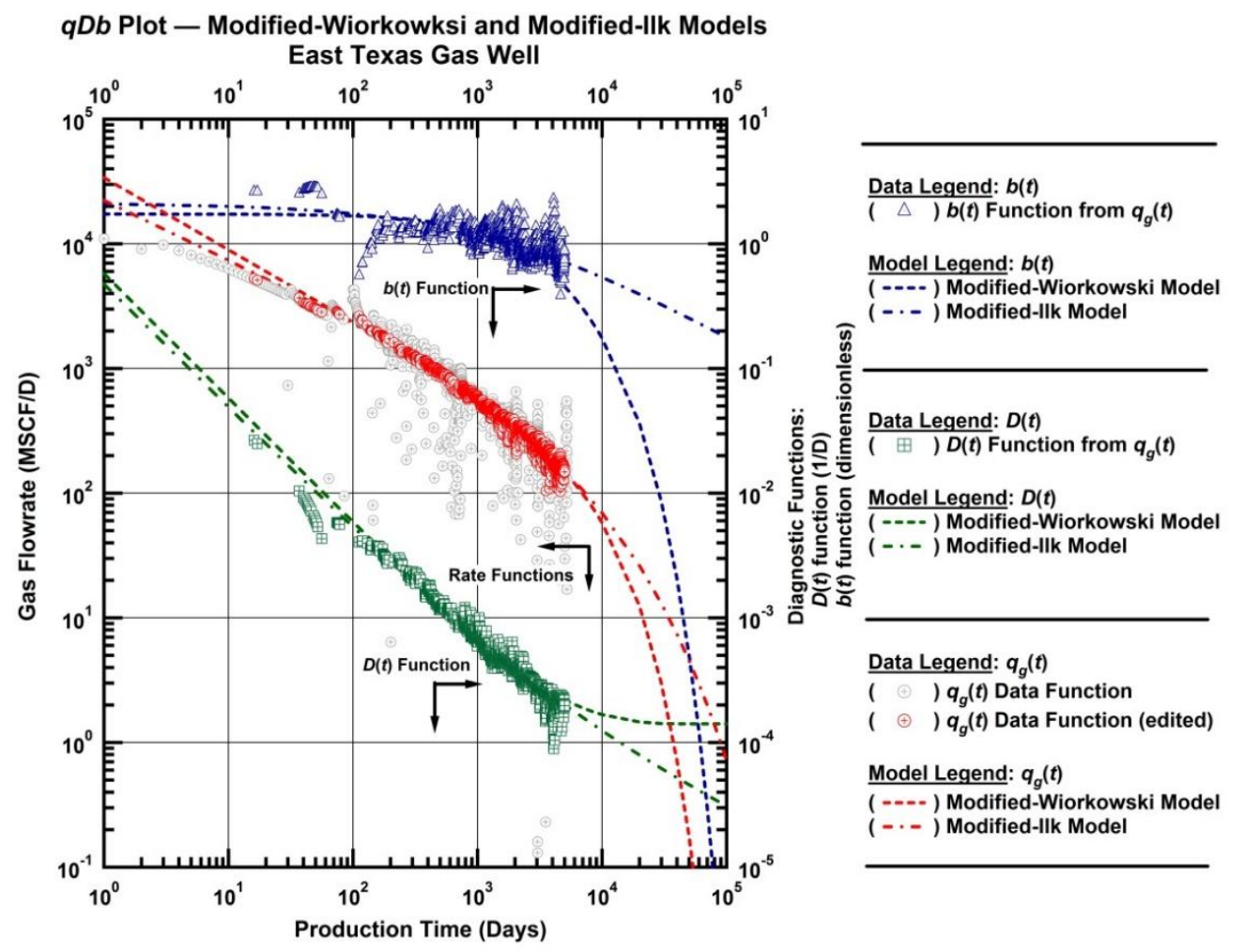

Figure 3.6 - (Log-log Plot): " $q D b "$ plot - Flow rate $\left[q_{g}(t)\right]$, Arps' Inverse Loss Ratio $[D(t)]$, and Arps' Loss Ratio Derivative $[b(t)]$ versus production time. the Modified-Wiorkowski Model, Modified-Ilk Model, Arps' Hyperbolic Model, Duong Model, Power Law Exponential Model, and the Logistic Growth Model diagnostic function matches for the East Texas Tight Gas Well.

In Fig. 3.7 we provide the time-rate and time-cumulative matches for all of the models considered in this work for the East Texas Tight Gas Well case. As suggested above, the Modified-Wiorkowski and the Modified-Ilk models provide the most conservative estimates of 30-year EUR $\left(E U R_{30 \mathrm{Yr}}\right)$ for the East Texas Tight Gas Well case. And while this particular case may not establish a "preference" for the Modified-Wiorkowski and the Modified-Ilk models, this case does establish that these models should be the "more conservative" models in the comparison group. 
In Table 3.6 we provide a summary of the results for all of the time-rate decline models for the East Texas Tight Gas Well - the $E U R_{30 Y r}$ values compare extremely well, but from observations made on Fig. 3.6, the longer term extrapolations (i.e., > 30 years) will vary significantly- however; this is of little consequence as we are only interested in near-term extrapolations (i.e., $<30$ years).

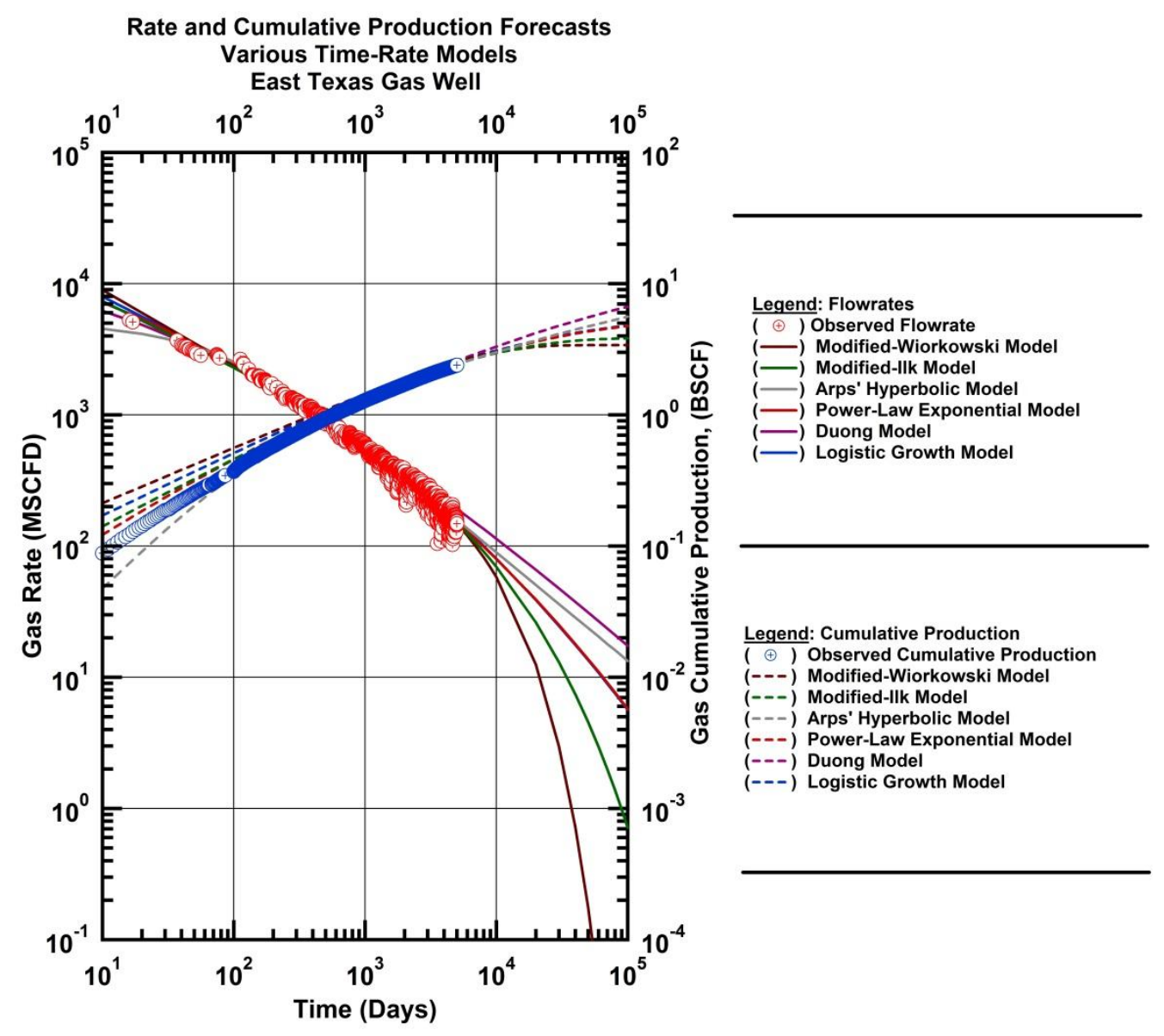

Figure 3.7 - (Log-log Plot): Modified Wiorkowski Model, Modified Ilk Model, Arps' Hyperbolic Model, Duong Model, Power Law Exponential Model and Logistic Growth Model 30-year EUR forecasts for East Texas Tight Gas Well -flow rate,(MSCFD) and cumulative production, (MSCF) versus production time,(Days) 
Table 3.6 - Summary of Decline Curve Analyses (EUR) for the East Texas Tight Gas Well. $\left(E U R_{30 \mathrm{Yr}}=2.8 \mathrm{BSCF}\right.$ from Rate Transient Analysis (RTA) $)$

\begin{tabular}{llc} 
Time-Rate Models & & $\begin{array}{c}E U R_{30 \mathrm{Yr}} \\
\text { (BSCF) }\end{array}$ \\
\hline Duong Model & & $3.31 \mathrm{BSCF}$ \\
Logistic Growth Model (LGM) & $3.09 \mathrm{BSCF}$ \\
Power-Law Exponential Model (PLE) & $3.03 \mathrm{BSCF}$ \\
Arps' Hyperbolic Model & $3.00 \mathrm{BSCF}$ \\
Modified-Wiorkowski Model (MWM) & $3.01 \mathrm{BSCF}$ \\
Modified-Ilk Model (MIM) & & $2.96 \mathrm{BSCF}$
\end{tabular}

Table.3.7 provides a summary of matched parameters.

Table 3.7 - Arps' Exponential, Arps' Hyperbolic, Duong, Logistic growth, Power Law and Wiorkowski Model matched parameters for numerical simulation case.

Arps' Hyperbolic Model

\begin{tabular}{|c|c|c|c|}
\hline $\begin{array}{c}q_{i} \\
\text { (MSCFD) }\end{array}$ & $D_{i}$ & $b$ & \\
\hline 6,000 & 0.11 & 2.60 & \\
\hline \multicolumn{4}{|c|}{ Logistic Growth Model } \\
\hline $\begin{array}{c}K \\
\text { (MSCFD) }\end{array}$ & $A$ & $n$ & \\
\hline $2.38 \times 10^{7}$ & 1500 & 0.62 & \\
\hline \multicolumn{4}{|l|}{ Duong Model } \\
\hline $\begin{array}{c}q_{t l} \\
\text { (MSCFD) }\end{array}$ & $A$ & $m$ & \\
\hline \multicolumn{4}{|c|}{ Modified Ilk Model } \\
\hline $\begin{array}{c}\bar{G}_{p} \\
(\mathrm{MSCFD})\end{array}$ & $\bar{D}_{\infty}$ & $\bar{D}_{i}$ & $\bar{n}$ \\
\hline $\begin{array}{c}3.04 \times 10^{6} \\
\text { PLE Model }\end{array}$ & $8.12 \times 10^{-7}$ & 0.00172 & 0.79 \\
\hline $\begin{array}{c}q_{i} \\
\text { (MSCFD) }\end{array}$ & $D_{i}$ & $n$ & $D_{\infty}$ \\
\hline $\begin{array}{c}900,920 \\
\text { Modified Wio }\end{array}$ & $\mathrm{del}^{4.48}$ & 0.0714 & $1 \times 10^{-7}$ \\
\hline $\begin{array}{c}\tilde{G}_{p} \\
(\mathrm{MSCFD})\end{array}$ & $\tilde{a}$ & $\tilde{D}_{i}$ & $\tilde{n}$ \\
\hline $2.23 \times 10^{6}$ & 0.99 & 0.00041 & 0.69 \\
\hline
\end{tabular}


We also generated flow rate and pressure model responses for the East Texas Tight Gas Well using an analytical model for a horizontally fractured well in an infinite acting homogenous reservoir after Ilk (2010). A very consistent history match of flow and pressure data was obtained with the "model" solution.

Fig. 3.8 gives a summary analysis plot of flow rate and pressure, and a 30-year forecast was generated using the matched model, which gave a 30-year EUR of 2.8 Bscf.

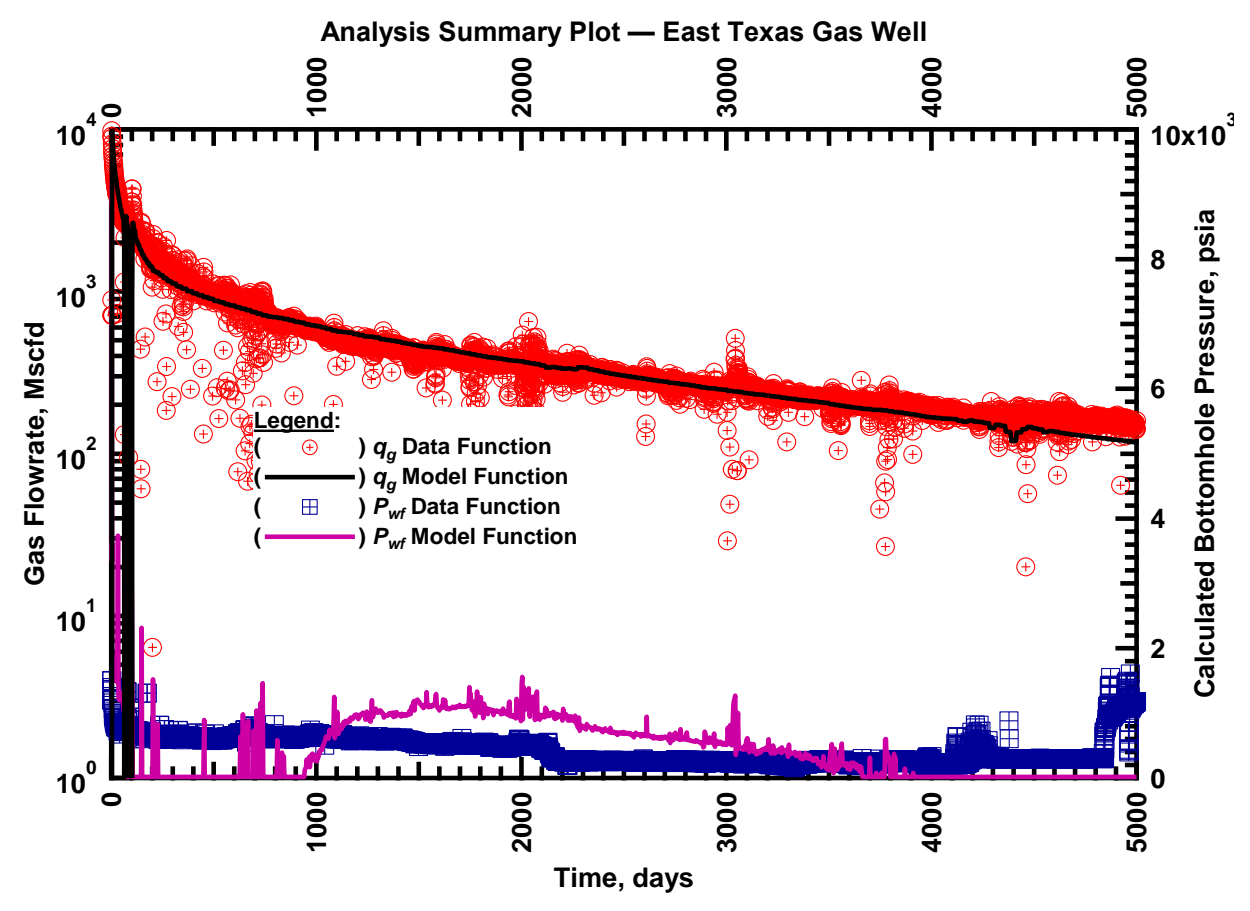

Figure 3.8 - (Semi-log Plot): East Texas Tight Gas Well analysis summary plot $-\mathrm{f}$ low rate,(MSCFD) and calculated bottomhole pressure, (psia) versus production time,(Days)

In Fig. 3.9 we present the "Continuous EUR" results versus production time for this case showing only the MWM and MIM models. In this case we use the "benchmark" 30-year EUR (EUR $\left.{ }_{30 \mathrm{Yr}}\right)$ obtained from model-based production analysis (typically referred to as "Rate Transient Analysis" or RTA). The MWM and MIM models match the $E U R_{30 \mathrm{Yr}}$ after about 5 years, then tend to slightly exceed this estimate for the remainder of the production period. This match should be considered more than sufficient given the conservative nature of the RTA method (RTA uses both pressure and rate data as well as a prescribed reservoir model, and tends to towards being conservative). 


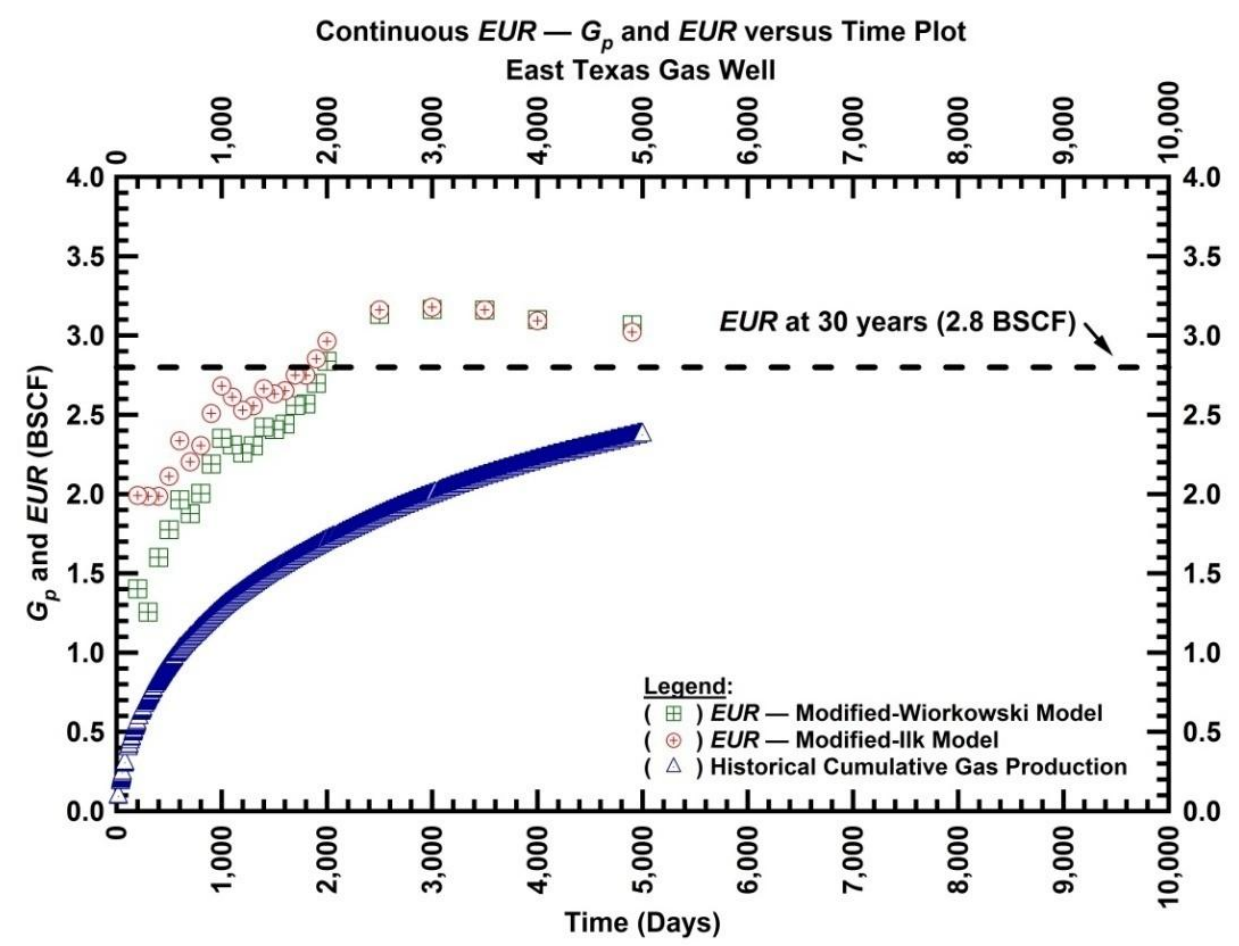

Figure 3.9 - (Cartesian Plot): "Continuous EUR" obtained using the Modified-Wiorkowski and Modified-Ilk models compared to the cumulative production (East Texas Gas Well).

\subsubsection{Field Example: Marcellus Shale Gas Well}

In this section we perform time-rate analysis of a shale gas well from the Marcellus Shale in Pennsylvania (USA). This case (Well 4) is a random selection taken from 55 Marcellus shale gas wells which were analyzed using the Modified-Wiorkowski and Modified-Ilk time-rate models. All of the Marcellus wells analyzed are horizontal wells with multiple transverse fractures. For the specific case of Well-4, we have approximately 800 days of production history. As with the East Texas Gas Well case (previous example), we begin our diagnostic analysis by "data editing," specifically, by removing data points that deviate from the dominant production trend. 
Table 3.8 shows reservoir and well parameters used to generate production data for this numerical simulation case.

Table 3.8 - Reservoir and fluid properties for Well-4

$\begin{array}{lll}\text { Reservoir Properties } & & \\ \quad \text { Net pay thickness, } h & & 156 \mathrm{ft} \\ \text { Formation permeability, } k & = & 22 \mu \mathrm{D} \\ \text { Wellbore Radius, } r_{w} & = & 0.35 \mathrm{ft} \\ \text { Formation compressibility, } c_{f}= & & 5.64 \times 10^{-6} \mathrm{psi}^{-1} \\ \text { Porosity, } \phi & = & 0.07(\mathrm{fraction}) \\ \text { Initial reservoir pressure, } p_{i} & = & 3493 \mathrm{psi} \\ \quad \text { Gas saturation, } s_{g} & = & 0.44 \text { fraction } \\ \quad \text { Skin factor, } s & = & 0.0127 \text { (dimensionless) } \\ \text { Reservoir temperature, } T_{r} & = & 138^{\circ} \mathrm{F} \\ \text { Fluid properties: } & & \\ \quad \text { Gas specific gravity, } \gamma_{g} & = & 0.568 \text { (air }=1) \\ \text { Hydraulically fractured well model } & \text { parameters: } \\ \text { Fracture half-length, } x_{f} & = & 124.6 \mathrm{ft} \\ \text { Number of fractures } & = & 36 \\ \text { Horizontal well length } & = & 3865 \mathrm{ft} \\ \text { Production parameters: } & & \\ \text { Production time, } t & = & 800 \text { days }\end{array}$

An inspection of historical production data of Well-4 (Fig. 3.10) shows significant anomalies in the early portion of the production history, most likely due to well clean-up (production of stimulation water) and production operations (choke management and some apparent shut-in sequences - probably due to offset operations and/or seasonal curtailments). In short, Well-4 is a challenging case, and while we are confident in our diagnostic approach, this case will have more uncertainty in the analysis and interpretation of the production performance.

Typically shale gas wells have very low permeability (on the scale of nanodarcies (nd)) and production histories are often very erratic with lots of large fluctuations especially in early time of well life. Fig. 3.10 gives a semi-log production history plot of Well-4. 


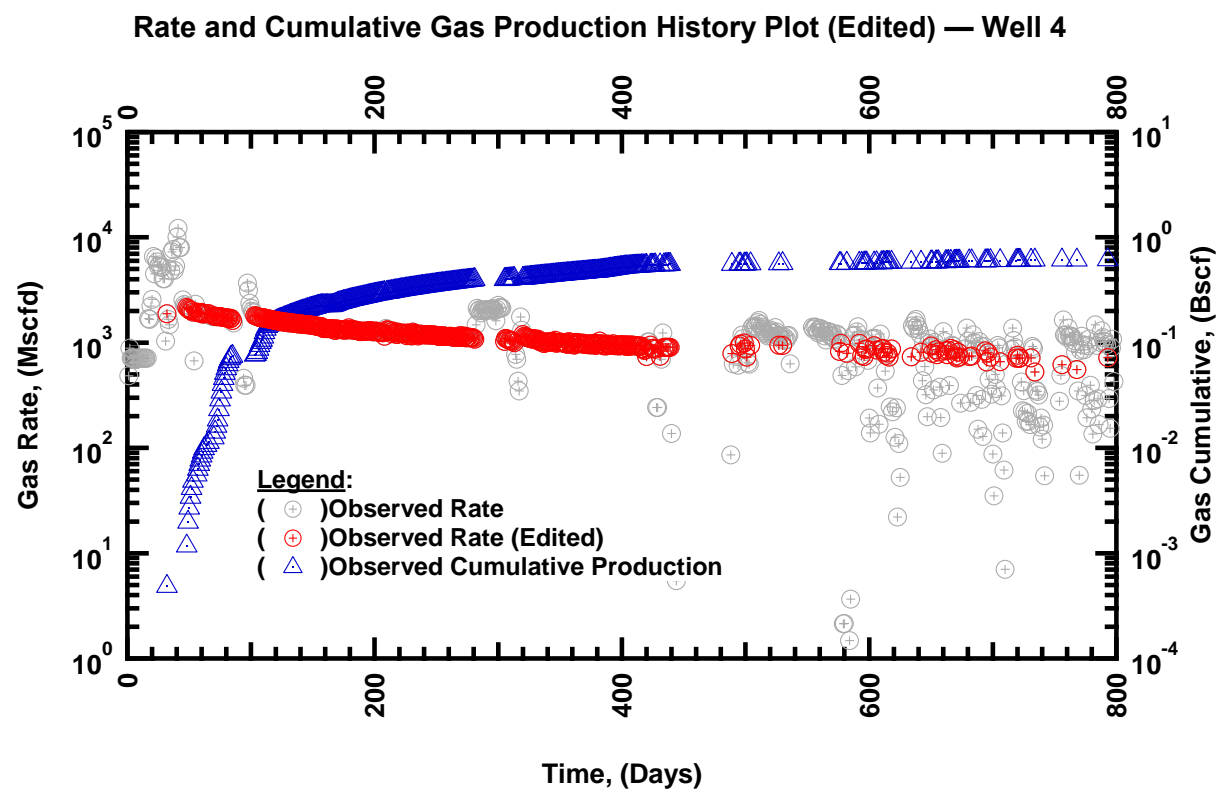

Figure 3.10 - (Semi-log Plot): Production history plot for Well-4 - flow rate,(MSCFD) and cumulative production, (BSCF) versus production time,(Days)

The Modified Wiorkowski and Modified Ilk Models were matched for 800 days of available production history of Well-4 to obtain 30-year EUR forecasts. Table 3.9 provides the parameter estimates for both models obtained from regression analysis, while Fig 3.11 shows 30-year EUR forecasts.

Table 3.9 - Modified Wiorkowski and Modified Ilk Models matched parameters for Well-4

\begin{tabular}{|c|c|c|c|}
\hline \multicolumn{4}{|c|}{$\frac{\text { Modified Wiorkow }}{\tilde{\tilde{a}}}$} \\
\hline $\begin{array}{c}\mathrm{c}_{p} \\
\text { (MSCF) }\end{array}$ & $\tilde{a}$ & $\tilde{n}$ & $\tilde{D}_{i}\left(\mathrm{D}^{-1}\right)$ \\
\hline $3.04 \times 10^{6}$ & 1 & 0.69 & 0.000414 \\
\hline \multicolumn{4}{|c|}{ Modified Ilk Model } \\
\hline$\overline{G_{p}}$ & & & \\
\hline (MSCF) & $\bar{D}_{\infty}$ & $\bar{n}$ & $\bar{D}_{i}\left(\mathrm{D}^{-1}\right)$ \\
\hline $2.23 \times 10^{6}$ & $8.1 \times 10^{-7}$ & 0.79 & 0.001719 \\
\hline
\end{tabular}

Relative to the diagnostic analyses based on the Modified-Wiorkowski and Modified-Ilk time-rate models we find reasonably good matches of $q_{g}(t), D(t)$, and $b(t)$ - and we would note that Well-4 appears to have a strong linear flow signature, i.e., $b(t) \approx 2$ for the period of $100-700$ days, with the caveat that this is a 
somewhat subjective interpretation of the $b(t)$ data profile shown using blue symbols as shown in Fig. 3.11.

Considering this apparent linear flow behavior (i.e., $b(t) \approx 2)$, we would also comment that neither the Modified-Wiorkowski Model (MWM) nor the Modified-Ilk Model (MIM) are capable of capturing the $b(t) \approx 2$ (and in fact, only the Arp's Hyperbolic or Modified-Hyperbolic relations are capable of doing so). This discussion is more to provide background and guidance on the application of the ModifiedWiorkowski and Modified-Ilk time-rate models — the matches for $q_{g}(t)$ are quite good, but are in something of a disagreement with the diagnostic model functions; $D(t)$, and $b(t)$.

Similar to previous comments, we note that due to the self-constraining nature of both the ModifiedWiorkowski and the Modified-Ilk models, we again obtain "conservative" forecasts and extrapolated reserves estimates.

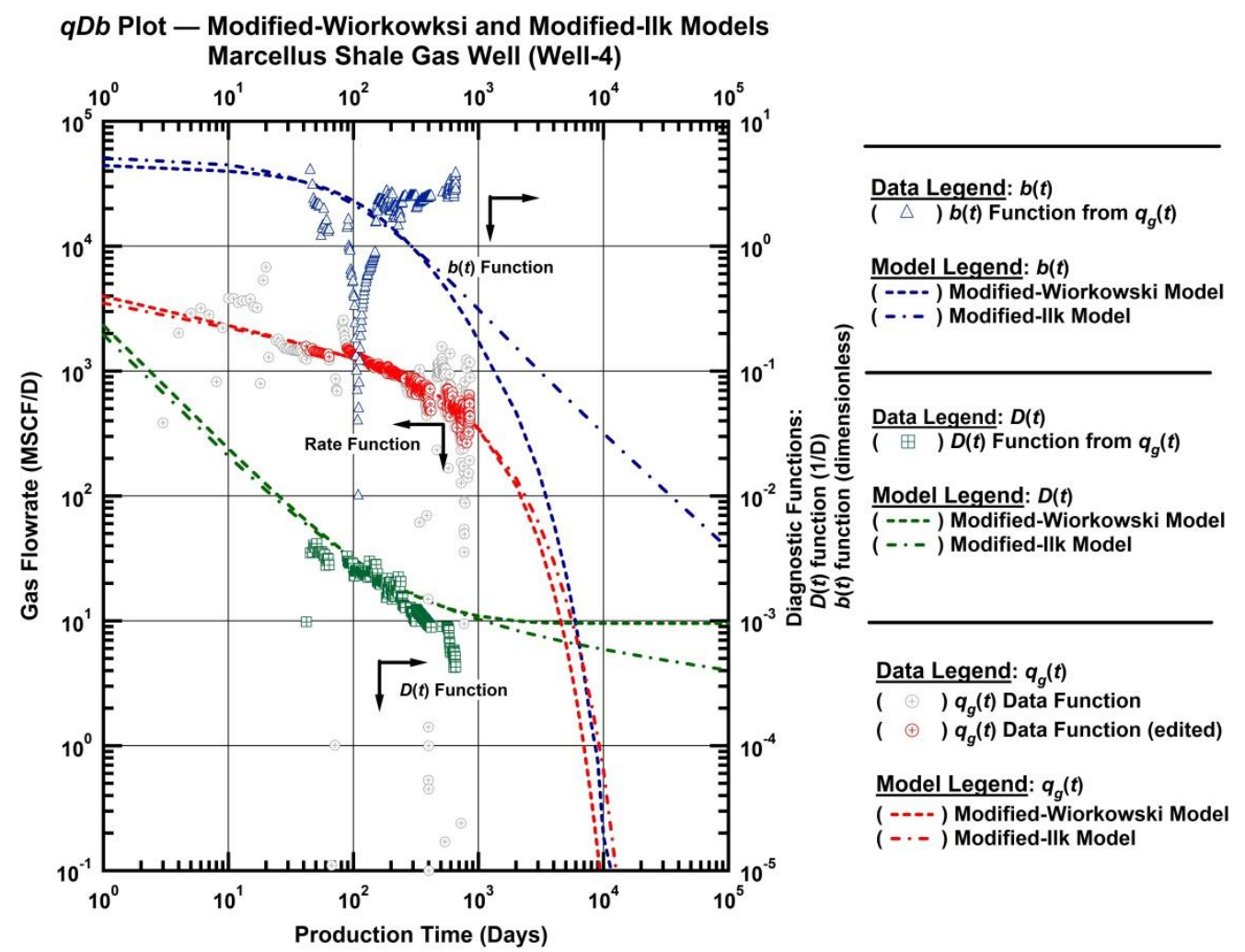

Figure 3.11 - (Log-log Plot): " $q D b "$ plot - Flowrate $\left[q_{g}(t)\right]$, Arps' Inverse Loss Ratio $[D(t)]$, and Arps' Loss Ratio Derivative $[b(t)]$ versus production time. The ModifiedWiorkowski Model, Modified-Ilk Model, Arps' Hyperbolic Model, Duong Model, Power Law Exponential Model, and the Logistic Growth Model diagnostic function matches for the Marcellus Shale Gas Well (Well-4). 
In Fig. 3.12 we provide the time-rate and time-cumulative matches for all of the models considered in this work for the Marcellus Shale Gas Well case (Well-4). In comparing models it is clear that the ModifiedWiorkowski and the Modified-Ilk models yield the most conservative estimates of 30-year EUR $\left(E U R_{30 Y \mathrm{Y}}\right)$. While these (very) conservative extrapolated trends match the time-rate and time-cumulative data quite well, the self-limiting growth nature of both the Modified-Wiorkowski and the Modified-Ilk models may have led to overly conservative production forecasts and estimated reserves.

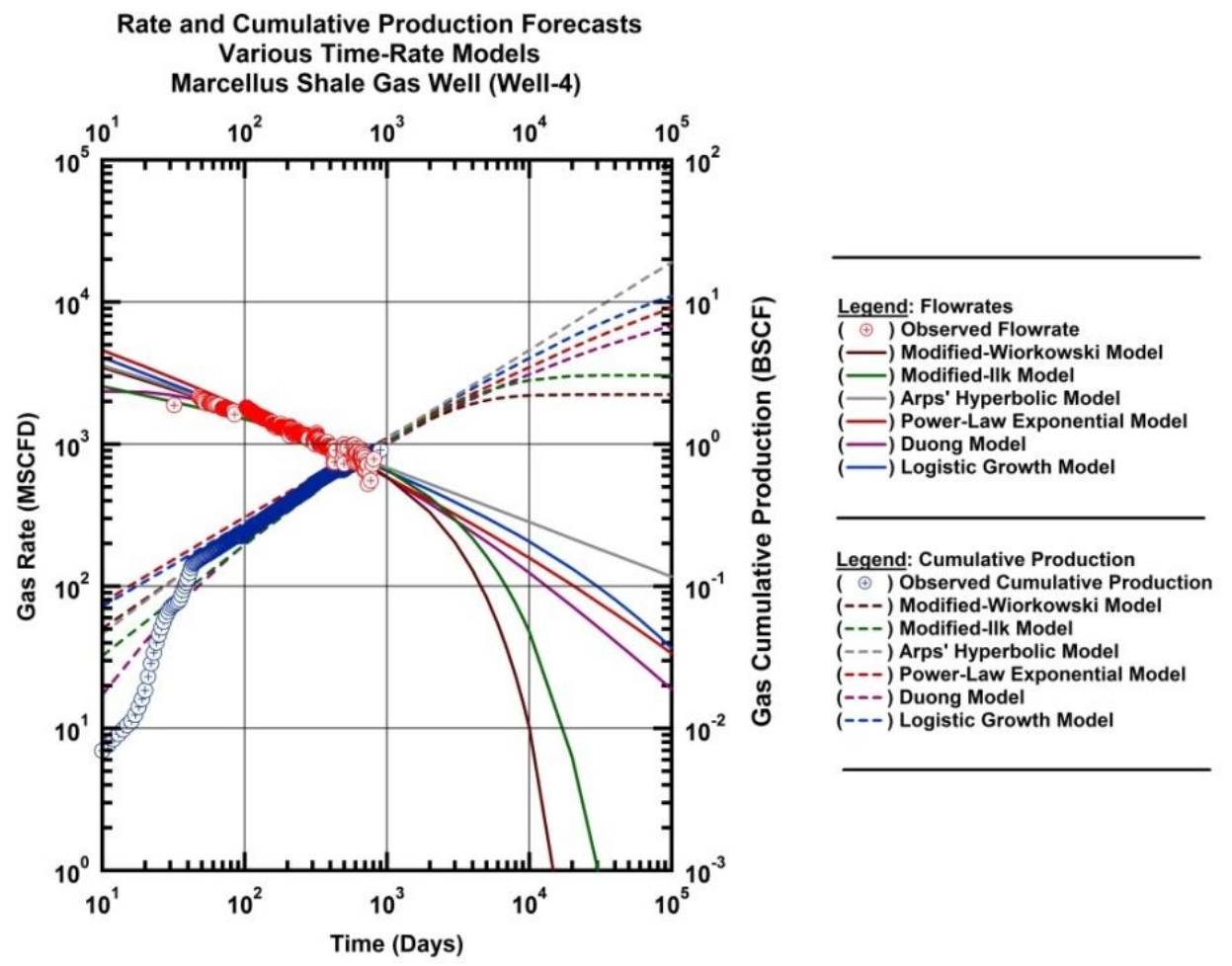

Figure 3.12 - (Log-log Plot): Modified-Wiorkowski Model, Modified-Ilk Model, Arps' Hyperbolic Model, Duong Model, Power Law Exponential Model and Logistic Growth Model 30-year EUR forecasts for the Marcellus Shale Gas Well (Well-4) - gas flowrate (MSCFD) and cumulative gas production (BSCF) versus production time (Days).

While not an indictment of the Modified-Wiorkowski Model (MWM) and Modified-Ilk Model (MIM) time-rate models; the Marcellus Shale Gas Well case (Well-4) does present a scenario where these new models may not be representative, even considering the fact that the $q_{g}(t)$ match using these models is quite good. In short, as with all time-rate analyses, we strongly recommend the diagnostic approach using the $q D b$ plot where the $q_{g}(t), D(t)$, and $b(t)$ functions must be matched by the model under consideration. 
If the proposed model does not effectively match all three functions, then some doubt/uncertainty in the validity of the proposed model must be stated for that particular case.

In Fig. 3.13 we present the "Continuous EUR" results versus production time for the Marcellus Shale Gas Well case (Well-4) showing all time-rate models. We again use the "benchmark" 30-year EUR (EUR $\left.R_{30 \mathrm{Yr}}\right)$ obtained from model-based production analysis (typically referred to as "Rate Transient Analysis" or RTA) as our EUR standard. As seen in Fig. 3.13, it is difficult to discern the "convergence" of the various time-rate models, but each of the models appears to be "trending" towards the $E U R_{30 \mathrm{Yr}}$ value of $2.52 \mathrm{BSCF}$ (with the noted exception of the Power-Law Exponential model which has exceeded this value). In fairness, the "Continuous EUR" approach can be difficult to apply. In this instance it is probably best to describe the nature of the Continuous EUR results as "indicative," rather than conclusive.

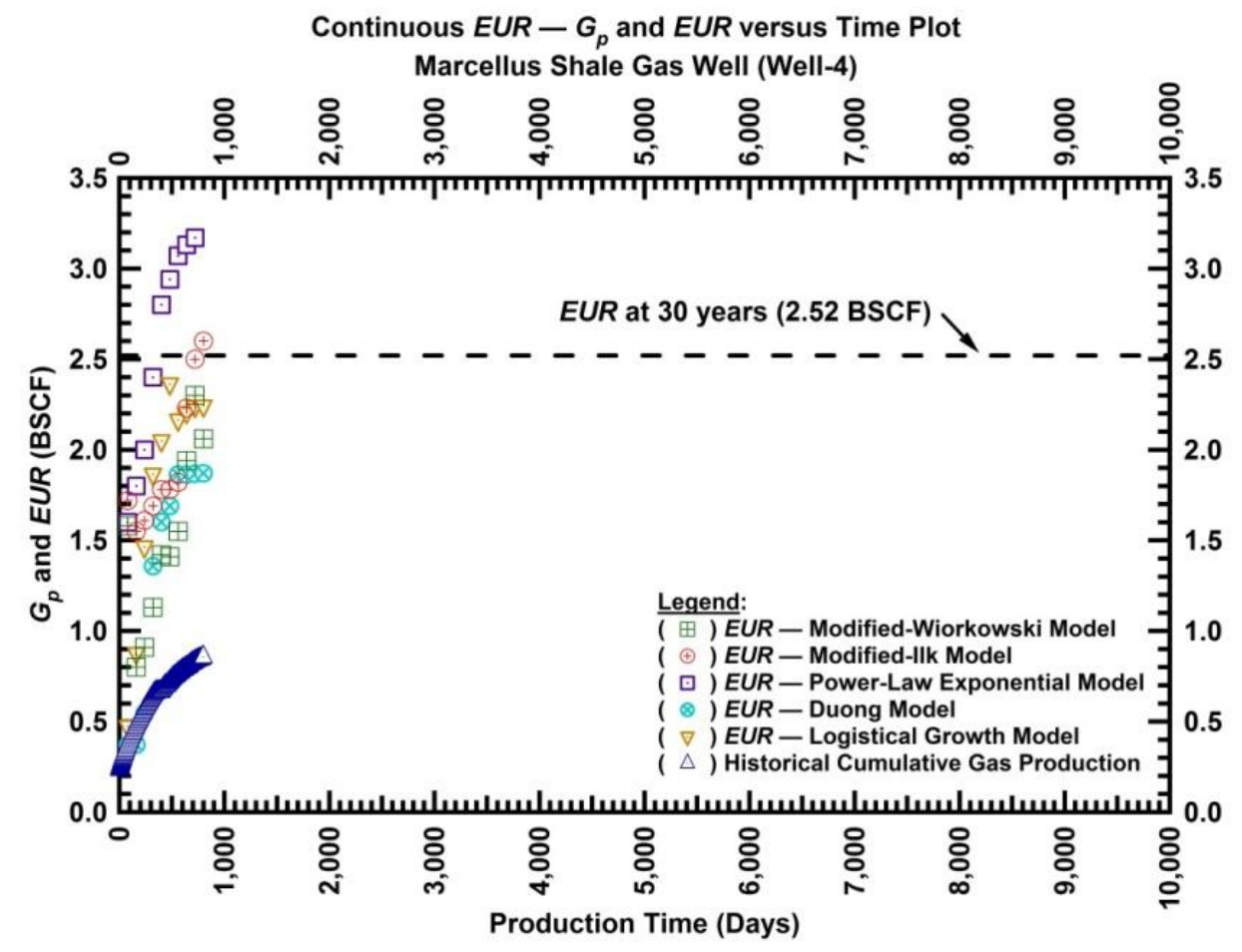

Figure 3.13 - (Cartesian Plot): "Continuous EUR" obtained using the Modified-Wiorkowski and Modified-Ilk models compared to the cumulative production (Marcellus Shale Gas Well (Well-4)). 
Table.3.10 provides a summary of matched parameters.

Table 3.10 - Arps' Exponential, Arps' Hyperbolic, Duong, Logistic growth, Power Law and Wiorkowski Model matched parameters for numerical simulation case.

Arps' Hyperbolic Model

\begin{tabular}{|c|c|c|c|}
\hline $\begin{array}{c}q_{i} \\
\text { (MSCFD) }\end{array}$ & $D_{i}$ & $b$ & \\
\hline 6,000 & 0.11 & 2.60 & \\
\hline \multicolumn{4}{|c|}{ Logistic Growth Model } \\
\hline $\bar{K}$ & & $n$ & \\
\hline (MSCFD) & $a$ & $n$ & \\
\hline $2.38 \times 10^{7}$ & 1500 & 0.62 & \\
\hline \multicolumn{4}{|l|}{ Duong Model } \\
\hline $\begin{array}{c}q_{t l} \\
(\mathrm{MSCFD})\end{array}$ & $a$ & $m$ & \\
\hline 1,200 & 1.73 & 1.16 & \\
\hline \multicolumn{4}{|c|}{ Modified Ilk Model } \\
\hline $\begin{array}{c}\bar{G}_{p} \\
(\mathrm{MSCFD})\end{array}$ & $\bar{D}_{\infty}$ & $\bar{D}_{i}$ & $\bar{n}$ \\
\hline \multicolumn{4}{|l|}{ PLE Model } \\
\hline $\begin{array}{c}q_{i} \\
\text { (MSCFD) }\end{array}$ & $D_{i}$ & $n$ & $D_{\infty}$ \\
\hline 900,920 & 4.48 & 0.0714 & $1 \times 10^{-7}$ \\
\hline \multicolumn{4}{|c|}{ Modified Wiorkowski Model } \\
\hline $\begin{array}{c}\tilde{G}_{p} \\
(\mathrm{MSCFD})\end{array}$ & $\tilde{a}$ & $\tilde{D}_{i}$ & $\tilde{n}$ \\
\hline $2.23 \times 10^{6}$ & 0.99 & 0.00041 & 0.69 \\
\hline
\end{tabular}

We also generated flow rate and pressure model responses of Well-4 for a horizontally fractured well in an infinite acting homogenous reservoir. A very consistent history match of flow and pressure data was obtained using these "model" solutions. Fig. 3. 14 gives a summary analysis plot of flow rate and pressure. A 30-year forecast of matched model was performed which gave a 30-year EUR of 2.52 Bscf. 


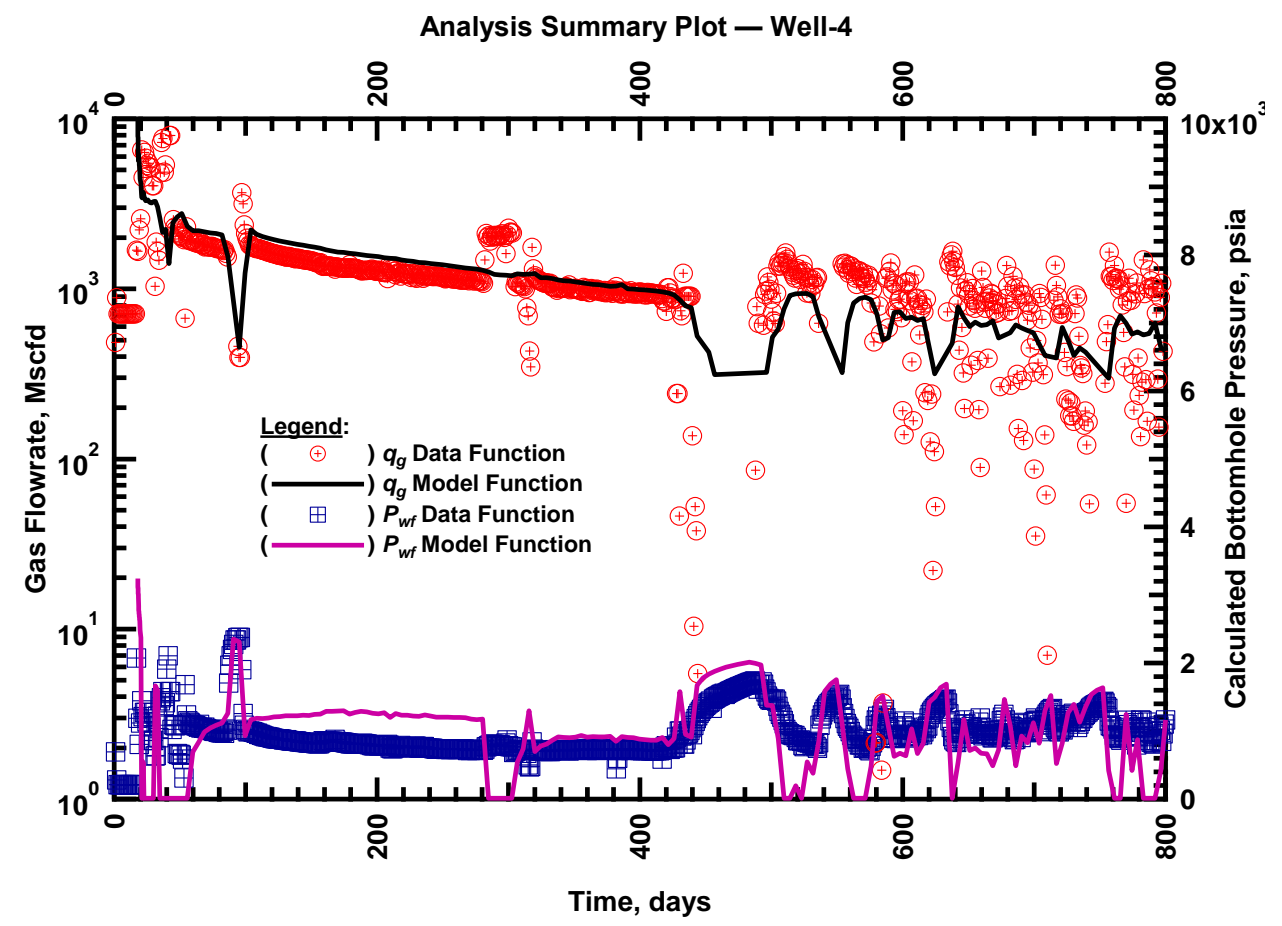

Figure 3.14 - (Semi-log Plot): Well-4 analysis summary plot - flow rate,(MSCFD) and calculated bottomhole pressure, (psia) versus production time,(Days)

\subsubsection{Field Example: Eagle Ford Gas Well}

In this section we perform time-rate analysis of an Eagle Ford Gas Well. Typically shale gas wells are horizontal wells with multiple transverse fractures and for this particular well, we have approximately 970 days of production data.

Table 3.11 shows reservoir and well parameters used to generate production data for this numerical simulation case. 
Table 3.11 - Reservoir and fluid properties for Eagle Ford Gas Well

\begin{tabular}{|c|c|c|}
\hline \multirow{2}{*}{\multicolumn{3}{|c|}{ Reservoir Properties }} \\
\hline & & \\
\hline Wellbore Radius, $r_{w}$ & $\begin{array}{l}= \\
=\end{array}$ & $0.3 \mathrm{ft}$ \\
\hline Porosity, $\phi$ & $=$ & 0.1 (fraction) \\
\hline Initial reservoir pressure, $p_{i}$ & $=$ & $7,000 \mathrm{psi}$ \\
\hline Gas saturation, $s_{g}$ & $=$ & 1.0 fraction \\
\hline Skin factor, $s$ & $=$ & 0.001 (dimensionless) \\
\hline Reservoir temperature, $T_{r}$ & $=$ & $212^{\circ} \mathrm{F}$ \\
\hline Fluid properties: & & \\
\hline Gas specific gravity, $\gamma_{g}$ & $=$ & $0.7($ air $=1)$ \\
\hline Hydraulically fractured well moc & $p a$ & ters: \\
\hline Fracture half-length, $x_{f}$ & $=$ & $192 \mathrm{ft}$ \\
\hline Number of fractures & $=$ & 30 \\
\hline Horizontal well length & $=$ & $3417 \mathrm{ft}$ \\
\hline Production parameters: & & \\
\hline Production time, $t$ & $=$ & 980 days \\
\hline
\end{tabular}

We again "edit" the flowrate data as shown in Fig. 3.15. As comment, these data are generally wellbehaved, with some production operations (most likely choke management) evident at early times. We again perform "diagnostic" time-rate analysis using the Modified-Wiorkowski and Modified-Ilk time-rate models - we note that the $q_{g}(t), D(t)$, and $b(t)$ data trends (symbols) are matched very well by their corresponding model trends (lines) - and although we do observe some discrepancies in the $D(t)$, and $b(t)$ data trends for times $>700$ days, these discrepancies are minor and could be attributed to derivative calculations (and/or slight data noise in the flowrate).

Typically shale gas wells have very low permeability (on the scale of nanodarcies) and production history is often very erratic with lots of large fluctuations especially in early time of well life. Fig. 3.15 gives a semi-log production history plot of Eagle Ford Gas Well. 


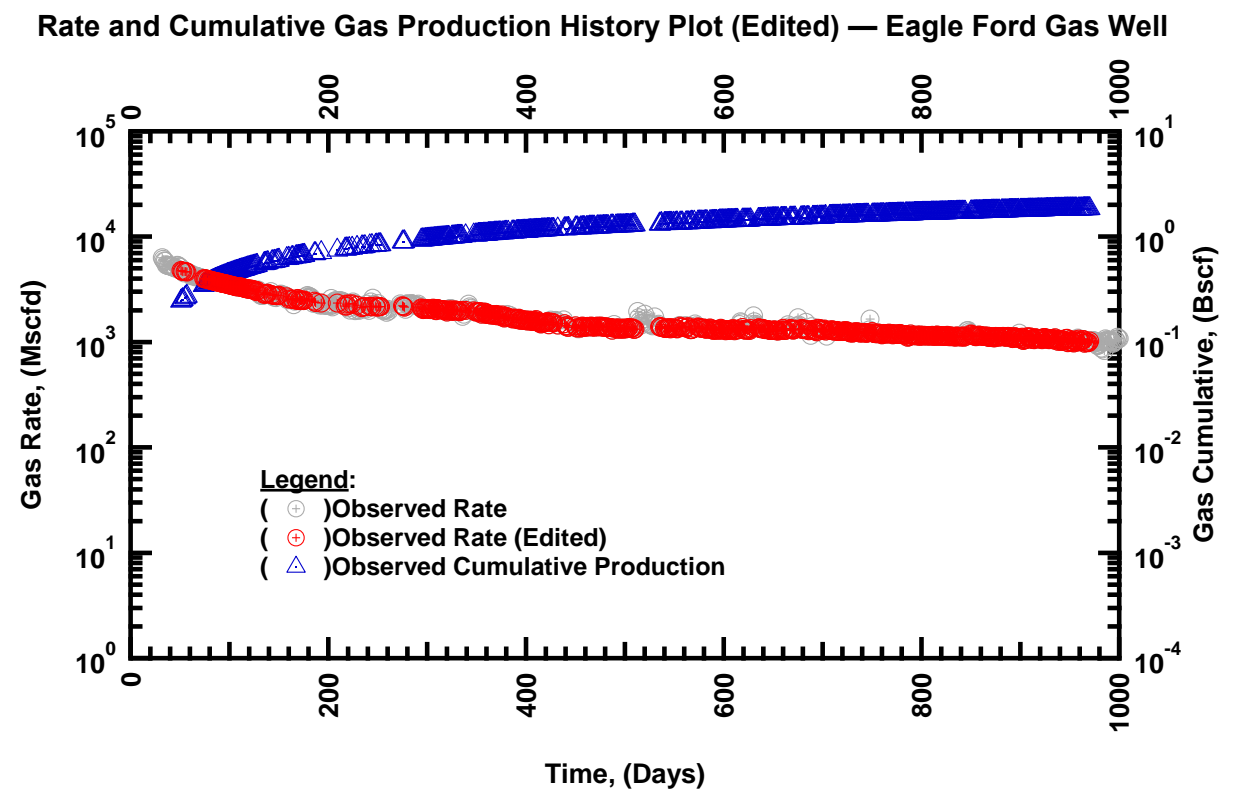

Figure 3.15 - (Semi-log Plot): Production history plot for Eagle Ford Gas Well - flow rate,(MSCFD) and cumulative production, (BSCF) versus production time,(Days)

Modified Wiorkowski and Modified Ilk Models were matched for 970 days of available production history of Eagle Ford Gas Well to obtain 30-year EUR forecasts. Table 3.12 gives matched parameters for both models.

Table 3.12 - Modified Wiorkowski and Modified Ilk Models matched parameters Eagle Ford Gas Well

\begin{tabular}{|c|c|c|c|}
\hline$\tilde{G}$ & & & \\
\hline $\begin{array}{c}\mathrm{u}_{p} \\
(\mathrm{MSCF})\end{array}$ & $\tilde{a}$ & $\tilde{n}$ & $\tilde{D}_{i}\left(\mathrm{D}^{-1}\right)$ \\
\hline $6.1 \times 10^{6}$ & 1 & 0.51 & 0.000131 \\
\hline \multicolumn{4}{|c|}{ Modified Ilk Model } \\
\hline$G_{p}$ & & & \\
\hline (MSCF) & $\bar{D}_{\infty}$ & $\bar{n}$ & $\bar{D}_{i}\left(\mathrm{D}^{-1}\right)$ \\
\hline $9.2 \times 10^{6}$ & $1 \times 10^{-7}$ & 0.55 & 0.00565 \\
\hline
\end{tabular}

Fig. 3.16 shows that the Modified Ilk Model provides more conservative EUR estimates as compared with the Modified Wiorkowski Model. From Fig. 3.16, we observe that the Eagle Ford Gas Well appears to be producing in the linear flow regime (see $b$ - parameter trend). 


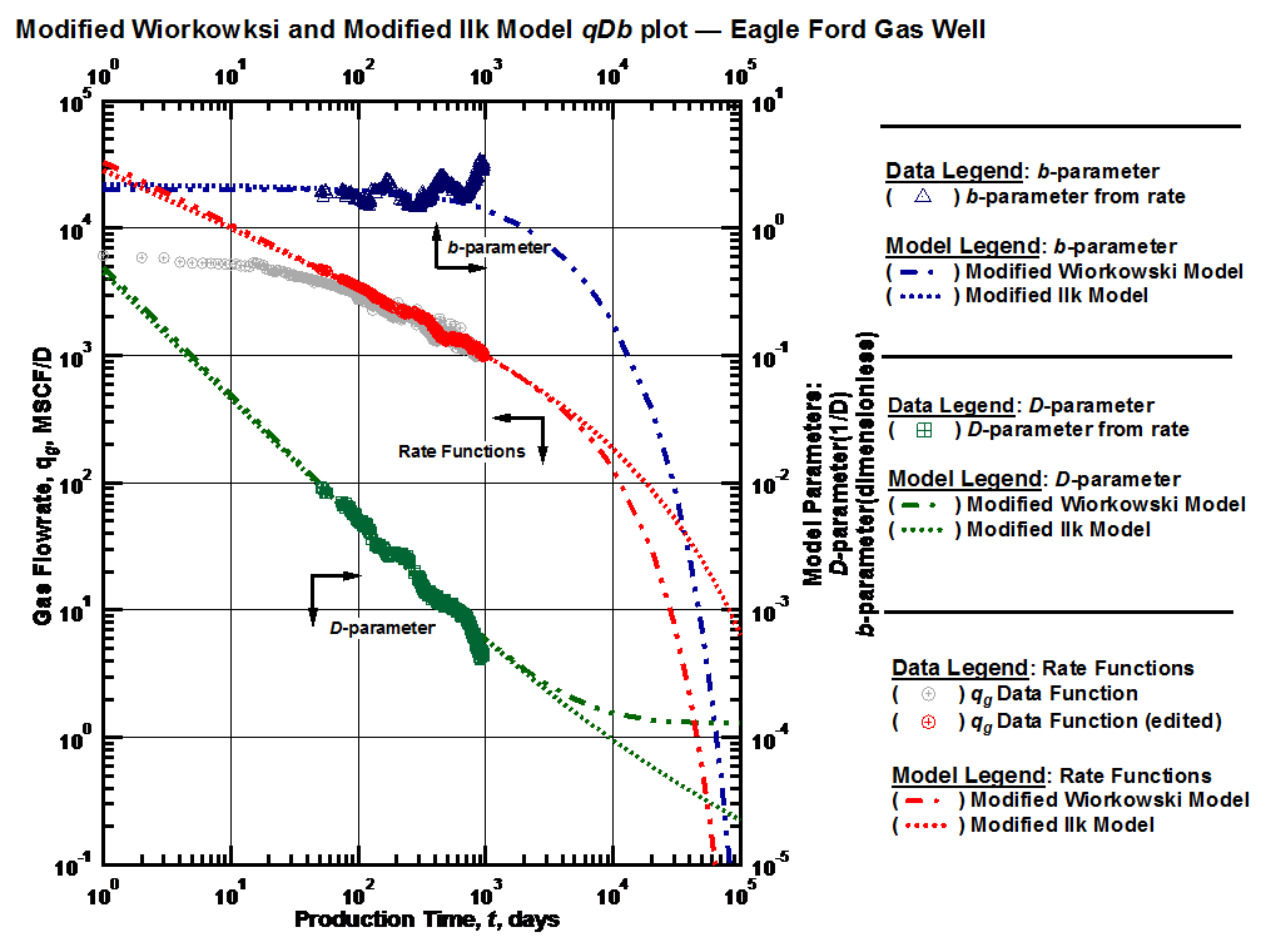

Figure 3.16 - (Log-log Plot): Flow rate, $D$ - and $b$-parameter versus production time. Modified Wiorkowski Model and Modified Ilk Model matches for Eagle Ford Gas Well.

In short, we believe that the Modified-Wiorkowski and Modified-Ilk time-rate models match the data trends well in Fig. 3.16, we would also note that the $D(t)$ data trend is essentially a straight-line trend, which corresponds to the concept of the Power-Law Exponential time-rate model. In addition, the selfconstraining nature of the Modified-Wiorkowski Model (MWM) and Modified-Ilk Model (MIM) timerate models suggests that these may again be overly conservative.

In Fig. 3.17 we provide the time-rate and time-cumulative matches for all of the models considered in this work for the Eagle Ford Gas Well case. We note that the Modified-Wiorkowski and Modified-Ilk timerate models are quite comparable to the Arps' Hyperbolic, the Duong, and the Logistic Growth time-rate models. We believe that the Power-Law Exponential time-rate and time-cumulative model match probably needs revision as we note that this model does not match the production performance at late times. 


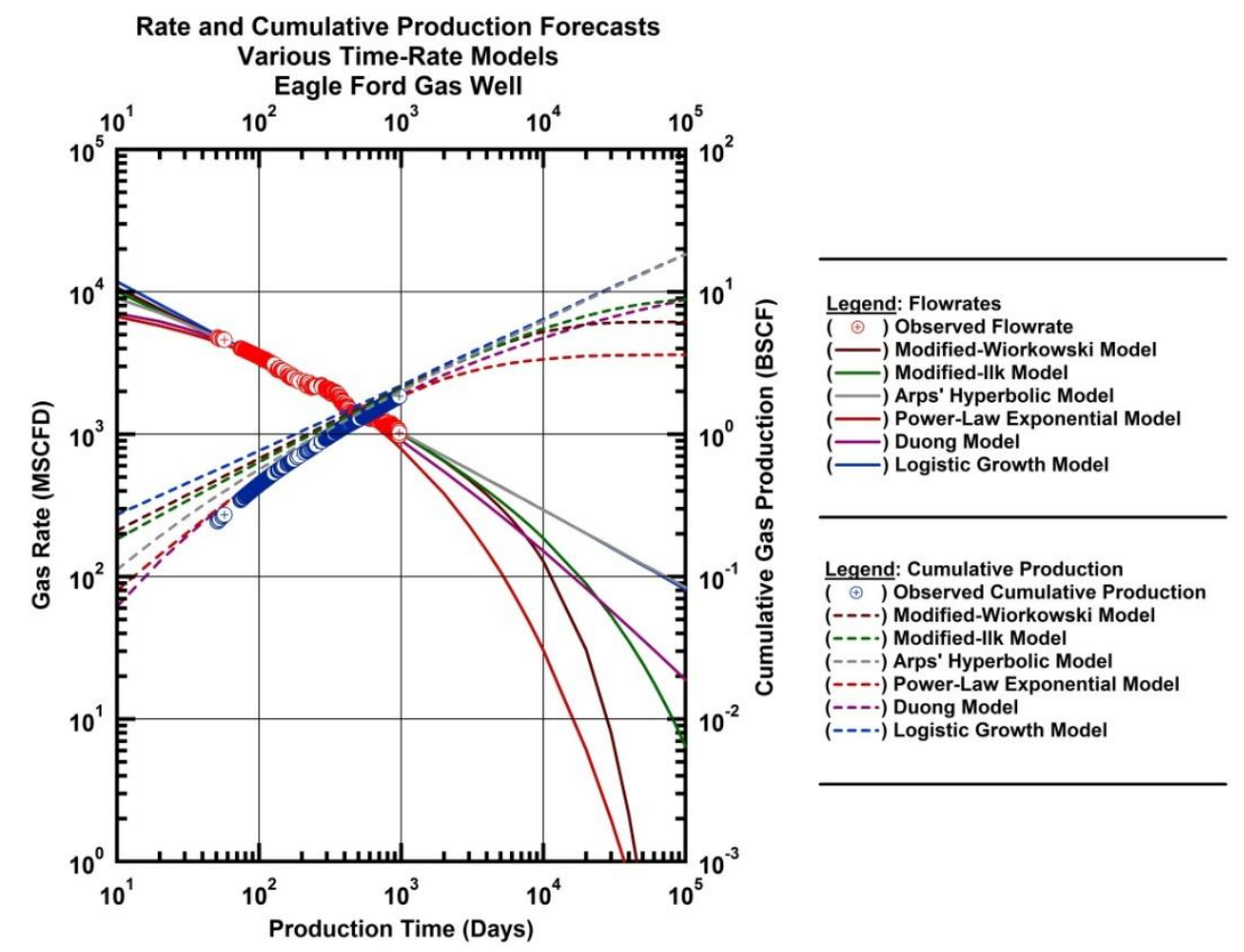

Figure 3.17 - (Log-log Plot): Modified-Wiorkowski Model, Modified-Ilk Model, Arps' Hyperbolic Model, Duong Model, Power Law Exponential Model, and the Logistic Growth Model time-rate model matches for the Eagle Ford Gas Well case - gas flowrate (MSCFD) and cumulative gas production (BSCF) versus production time (Days).

Table 3.13 - Summary of Decline Curve Analyses (EUR) for the Eagle Ford Gas Well case.

Time-Rate Models

Duong Model

Logistic Growth Model (LGM)

Power-Law Exponential Model (PLE)

Arps' Hyperbolic Model

Modified-Wiorkowski Model (MWM)

Modified-Ilk Model (MIM)
$E U R_{30 \mathrm{Yr}}$

(BSCF)

4.80 BSCF

6.50 BSCF

$3.40 \mathrm{BSCF}$

6.20 BSCF

$5.32 \mathrm{BSCF}$

$5.55 \mathrm{BSCF}$

Table.3.14 provides a summary of the matched parameters for this case. 
Table 3.14 - Arps' Exponential, Arps' Hyperbolic, Duong, Logistic growth, Power Law and Wiorkowski Model matched parameters for Eagle Ford Gas Well.

Arps' Hyperbolic Model

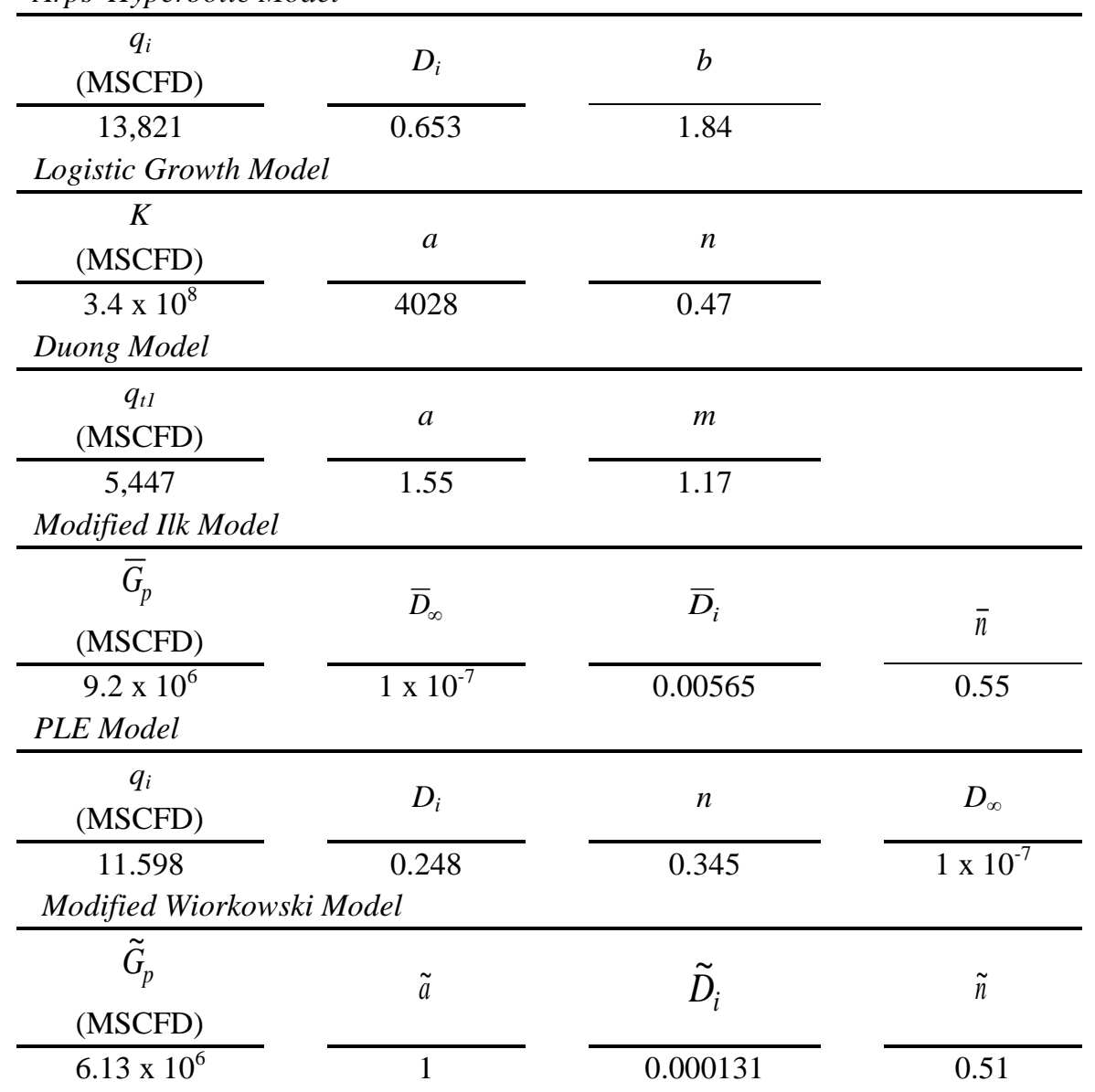

As noted, the results are comparable for all cases (with the exception of the Power-Law Exponential Model). Also, Rate Transient Analysis (RTA) was not performed for this case due a lack of reservoirspecific data.

We also generated flow rate and pressure model responses for the Eagle Ford Gas Well case using a horizontally fractured well in an infinite acting homogenous reservoir. A very consistent history match of flow and pressure data was obtained with the "model" solution. Fig. 3.18 provides a summary analysis plot of flow rate and pressure. A 30-year using the RTA model gave an EUR of 2.52 Bscf. 


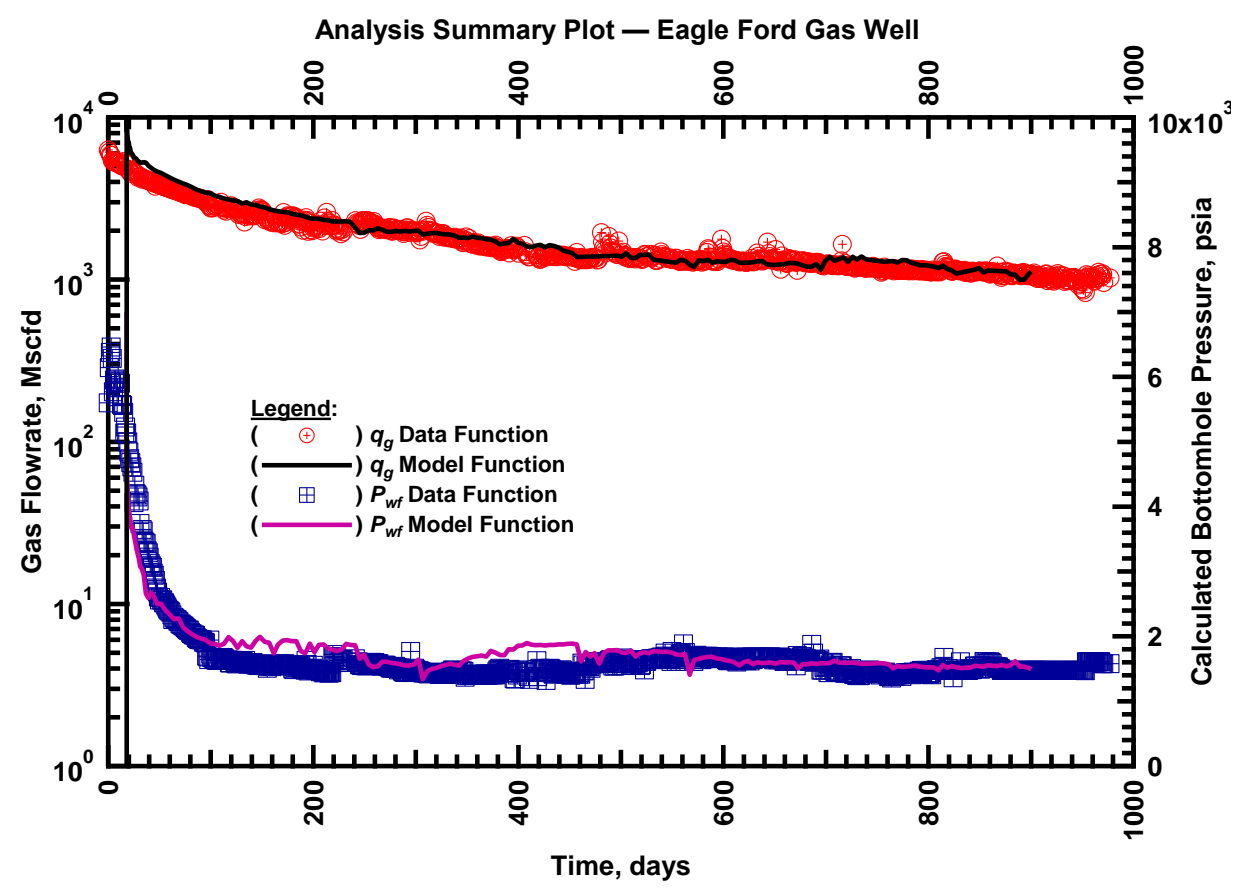

Figure 3.18 - (Semi-log Plot):Eagle Ford Gas Well analysis summary plot -flow rate, (MSCFD) and calculated bottomhole pressure, (psia) versus production time,(Days) 


\section{CHAPTER IV}

\section{PARAMETRIC CORRELATIONS OF WELL/RESERVOIR PROPERTIES AND PRODUCTION METRICS}

This section presents a methodology to integrate parameters from proposed time-rate relations with completion/reservoir parameters from model based production analysis. We investigate parametric correlations for Modified Wiorkowski Model and Modified Ilk Model for fracture conductivity, formation permeability, fracture half-length and 30 year EUR values. We provide a theoretical consideration to the methodology using data generated from different numerical simulation cases for flow from a horizontal well with multiple transverse fractures in a low/ultra-low permeability reservoir.

For this study, we assume that all other completion and reservoir parameters are kept fairly constant for all cases. For all cases considered, we use the same reservoir parameters and production constraints like initial reservoir pressure and temperature, saturations, flowing bottomhole pressure, number of fractures, drainage area, well-length etc. to narrow the unknown parameter to fundamental reservoir/completion parameters being investigated for parametric correlations with time-rate relations.

\subsection{General Methodology}

We model a horizontal well with multiple transverse fractures in a low permeability reservoir for numerical simulation cases. A typical numerical simulation grid is shown in Fig. 4.1.

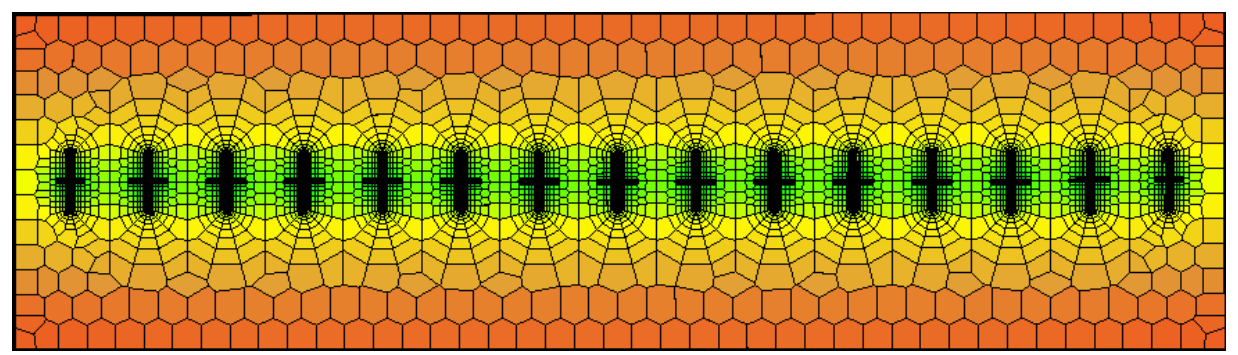

Figure 4.1 - Diagram of the numerical simulation model showing horizontal well and multiple transverse fractures.

For each case we make 15 simulation runs and perform time-rate analysis with Modified Wiorkowski and Modified Ilk models. We use Bourdet algorithm (Bourdet et al. 1989) to calculate the $D$ - and $b$-parameters to help in matching the production data. We provide $\log -\log$ plots of gas rate $q_{g}, D$ - and $b$-parameters against production time to show the quality of model matches. 
Once we obtain satisfying matches of time-rate models, the next step is to study the relationship between individual model parameters and the reservoir parameter being considered along with 30 year EUR. We perform a cross-plot analysis of the individual rate decline model parameters with reservoir parameters and identify respective correlating parametric functions.

We intend to find each reservoir parameter in terms of the rate decline model parameters. For example:

$$
F_{r}=f(p)
$$

And

$$
E U R_{30 y r}=f(p)
$$

Here, $\mathrm{F}_{\mathrm{r}}$ is the reservoir parameter and $p$ is the rate decline model parameter under consideration.

After establishing individual correlating functions we find multivariate correlating functions for reservoir parameter and 30 year EUR for each case by finding suitable combinations of the correlating functions. This serves to provide relations which can predict reservoir parameters directly from rate decline model parameters.

The integrating parametric correlation functions can be written as:

$$
F_{r}=f\left(p, q, r_{\ldots}\right)
$$

And

$$
E U R_{30 y r}=f\left(p, q, r_{\ldots}\right)
$$

$p, q$, and $r$ are sample rate decline model parameters being considered.

Finally we provide cross-plots of calculated and observed reservoir parameters to assess the quality of parametric correlating functions.

\subsection{Fracture Conductivity - Parametric Correlations}

In this section we will investigate the parametric correlation between two proposed models and fracture conductivity for 15 numerical simulation cases with fracture conductivity varying between 0.005-0.7 md$\mathrm{ft}$. The model input parameters for numerical simulation case are given below. 
Table 4.1 - Reservoir and fluid properties for numerical simulation case (horizontal well with multiple transverse fractures) with varying fracture conductivity.

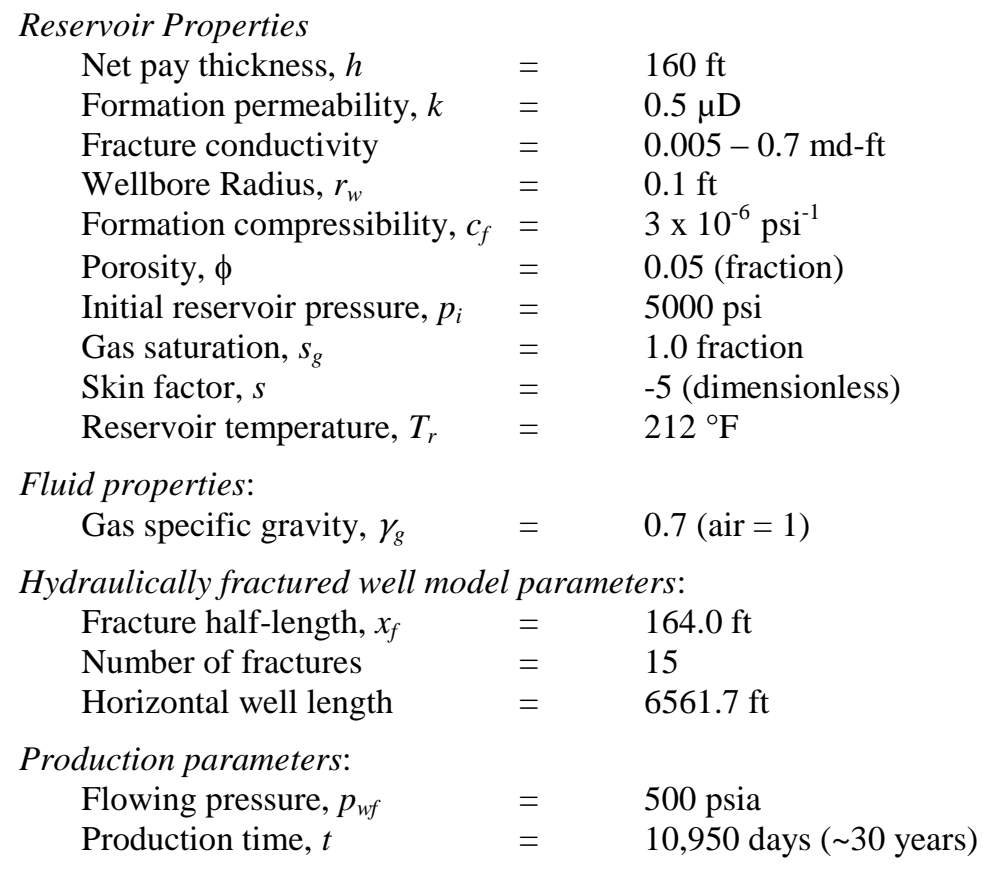

In the following two sections, we present parametric correlations for both models respectively.

\subsubsection{Modified Wiorkowski Model - Parametric Correlations}

In this section we investigate parametric correlating functions between parameters of Modified Wiorkowski Model and 15 numerical simulation cases with varying fracture conductivities. The gas flowrate $q_{g}, D$ - and $b$-parameter plots are given below in Figs. 4.2, 4.3, and 4.4 respectively. The diagnostic functions indicate transient and transition flow regimes followed by boundary-dominated flow in late time part of data. However, in this analysis, we focus on the behavior of the linear flow regime.

Fig.4.3 shows a plot of $b$ - parameter values for various fracture conductivity cases. For a typical low permeability well, $b$ - parameter has a value of 2 for transient flow regime for large fracture conductivity cases. For our study, we have considered relatively low fracture conductivity values which give a $b$ parameter value of 3 (0.7 md-ft) for highest fracture conductivity case while a value of 10 (0.005 md-ft) for lowest fracture conductivity case. Fig. 4.3 shows a dominant transient/transition flow regime characterized by a negative slope straight line decline of $D$ - parameter on a log-log plot. We observe a slight deviation of trend and change in slope at around 1,000 days. It should be noted that for all cases Modified Wiorkowski Model $D$ - parameter approaches a constant value at around 20,000 days. This feature exhibits the inherent self-growth limiting feature of Modified Wiorkowski Model that makes this 
model suitable to match boundary-dominated flow regime. Table 4.2 shows a constant value of unity of parameter $\tilde{a}$ for all varying fracture conductivity cases which leads us to its subsequent exclusion from parameter correlation process.

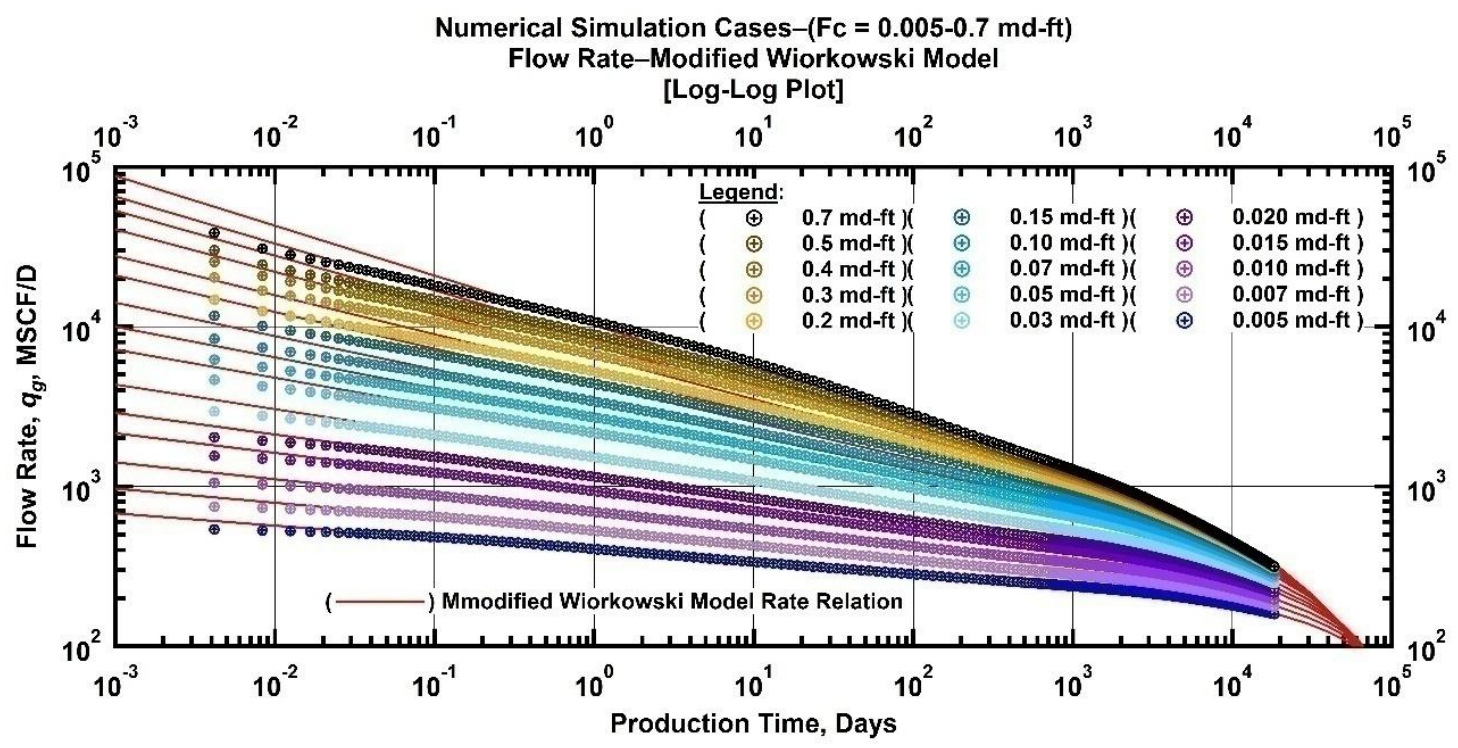

Figure $4.2-\quad$ (Log-log Plot): Flow rate $\left(q_{g}\right)$ versus production time. Modified Wiorkowski model matches of 15 numerical simulation cases.

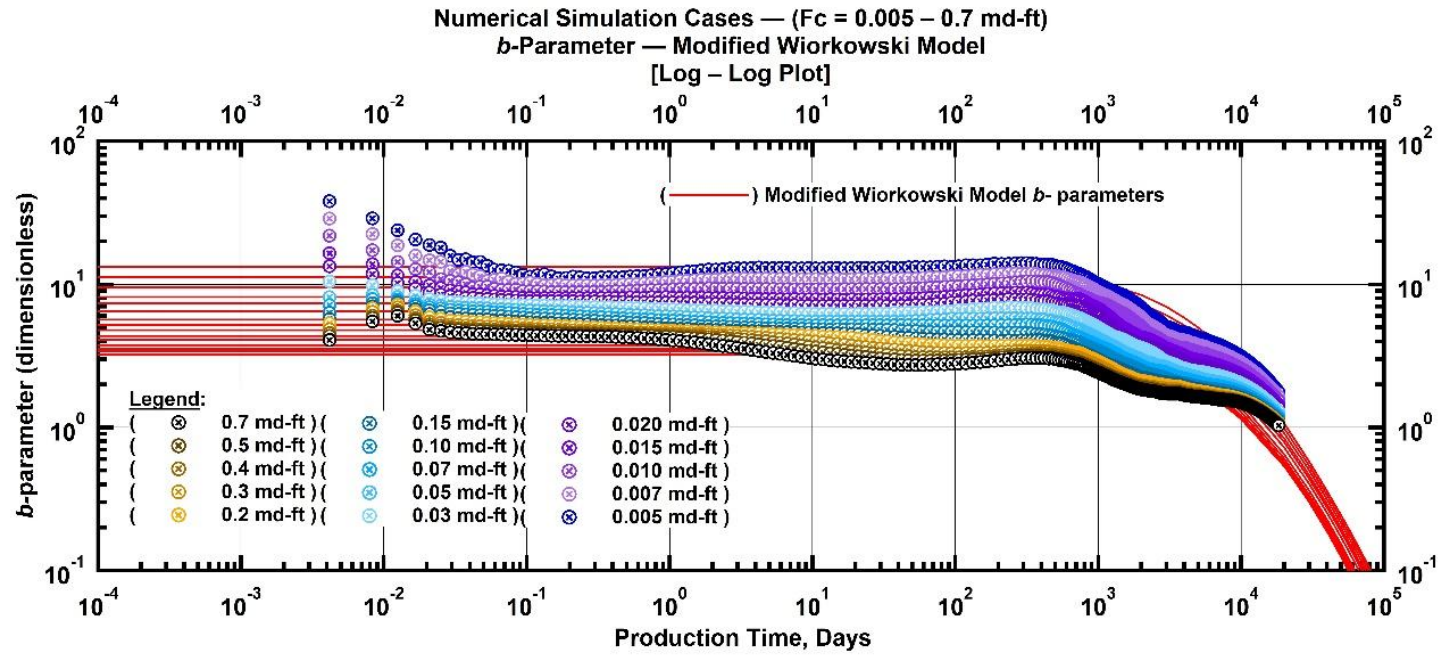

Figure $4.3-\quad($ Log-log Plot): $b$-parameter versus production time. Modified Wiorkowski model model matches of 15 numerical simulation cases. 


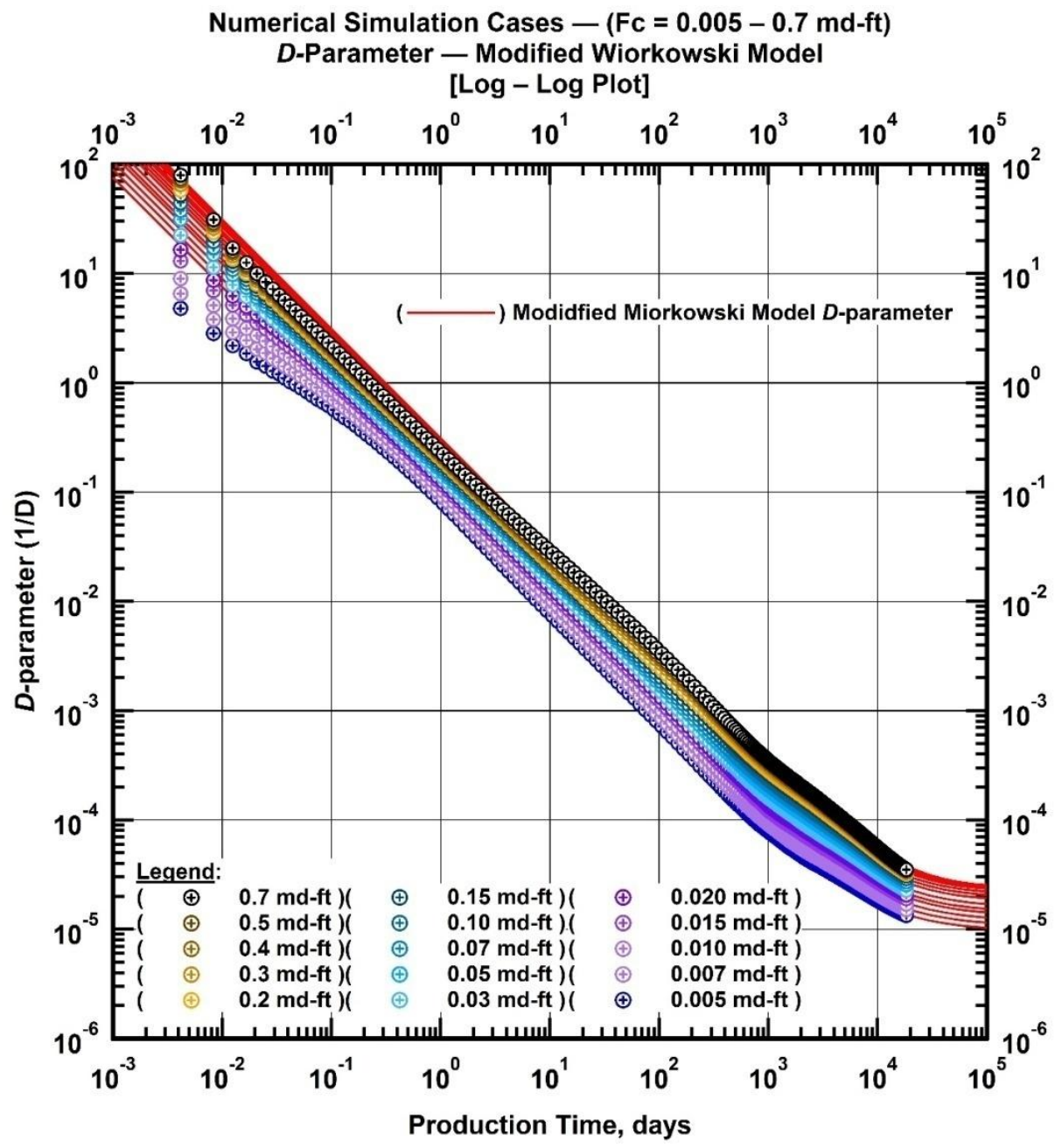

Figure $4.4-\quad$ (Log-log Plot): $D$ - parameter versus production time. Modified Wiorkowski model matches of 15 numerical simulation cases.

Table 4.2 shows the matching model parameters. 
Table 4.2 - Modified Wiorkowski Model parameters. Model matches to 15 numerical simulation cases.

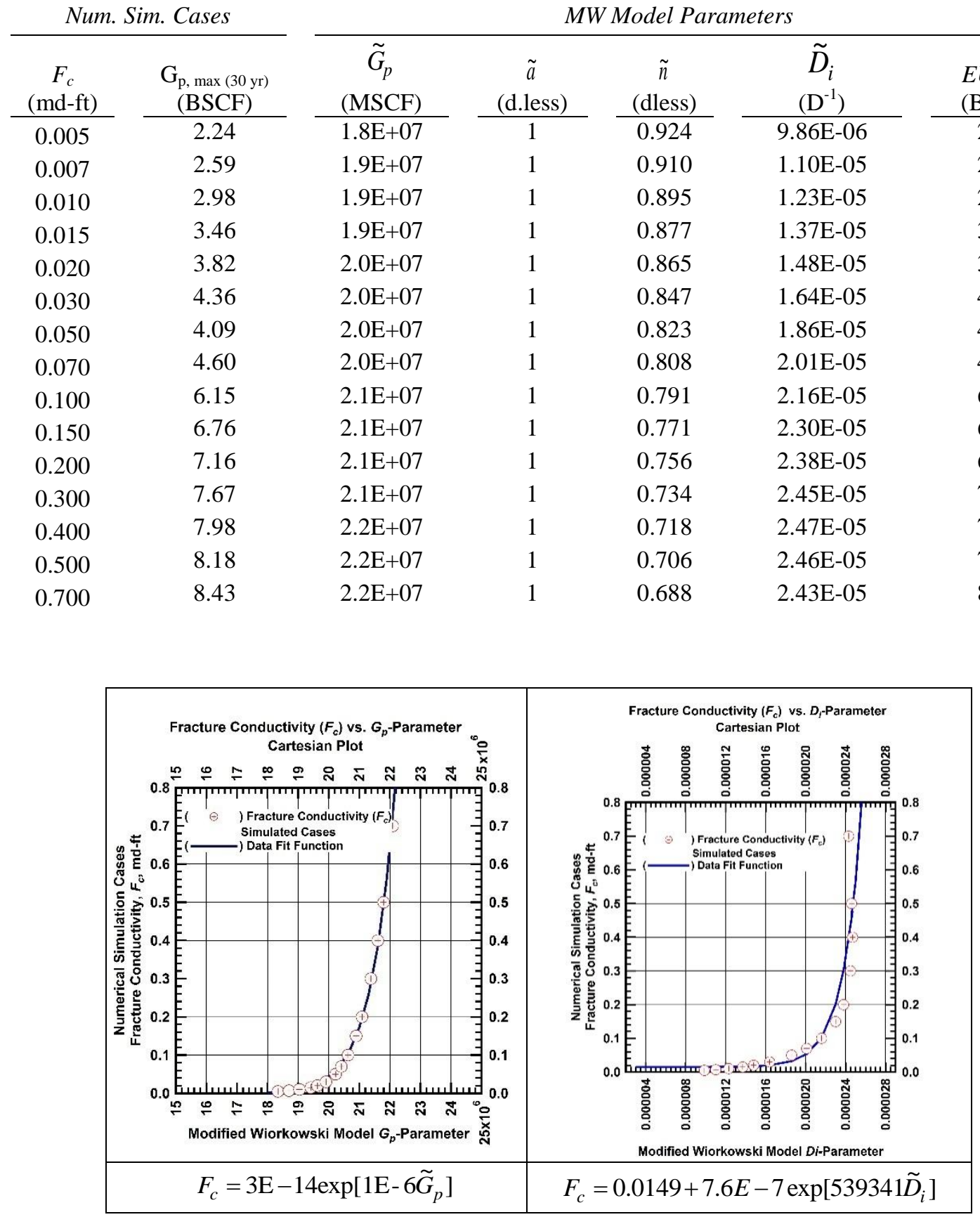




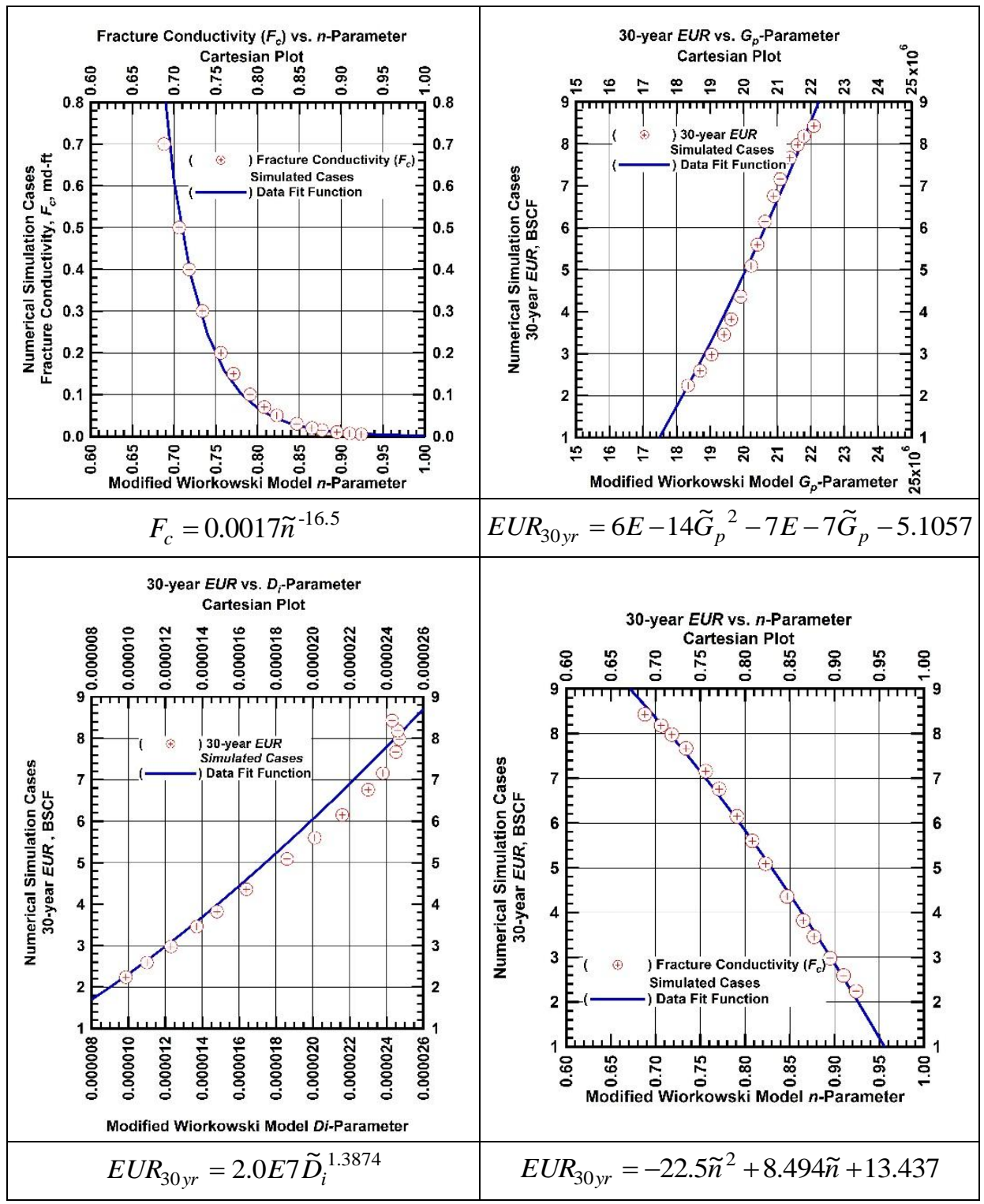

Figure 4.5 - Cross-plots showing relationship between Modified Wiorkowski model parameters and numerical simulation case fracture conductivity $\left(F_{c}\right)$ and 30 year EUR estimates.

Fig. 4.5 shows the cross-plots of each considered parameter of Modified Wiorkowski Model parameter for 15 simulation cases against fracture conductivity and 30-year EUR respectively. We also fit a simple parametric function to cross-plot to determine the underlying correlating function for individual parameters. Fig. 4.5 shows the cross-plots along with respective correlating functions.

Finally, we relate reservoir parameters with Modified Wiorkowski model parameters by proposing parametric correlations based on the correlating functions we identified in Fig. 4.4. Fig.4.5 shows that 
fracture conductivity can be correlated to model parameters by a combination of power and exponential functions. We propose the correlating function as:

$$
F_{c}=a_{01} \tilde{G}_{p}^{a_{02}} \tilde{n}^{a_{03}} \exp \left[{ }^{a_{04}} \tilde{D}_{i}\right]
$$

Here, $a_{01}, a_{02}, a_{03}$ and $a_{04}$ are coefficients to be determined through least square regression. Similarly we propose the following correlating function to estimate the 30 year $E U R\left(E U R_{30 y r}\right)$.

$$
E U R_{30 y r}=a_{01} \widetilde{D}_{i}^{a_{02}}-a_{03} \widetilde{G}_{p}^{a_{04}} \ln [\tilde{n}]
$$

Fig. 4.6 shows the resulting model fits for proposed parametric correlations. It can be observed that the proposed correlating functions provide a reliable estimate of the reservoir properties $\left(F_{c}\right.$ and $E U R_{30}$ yr $)$ for the case of a well within the same reservoir system with similar completion and production constraints.

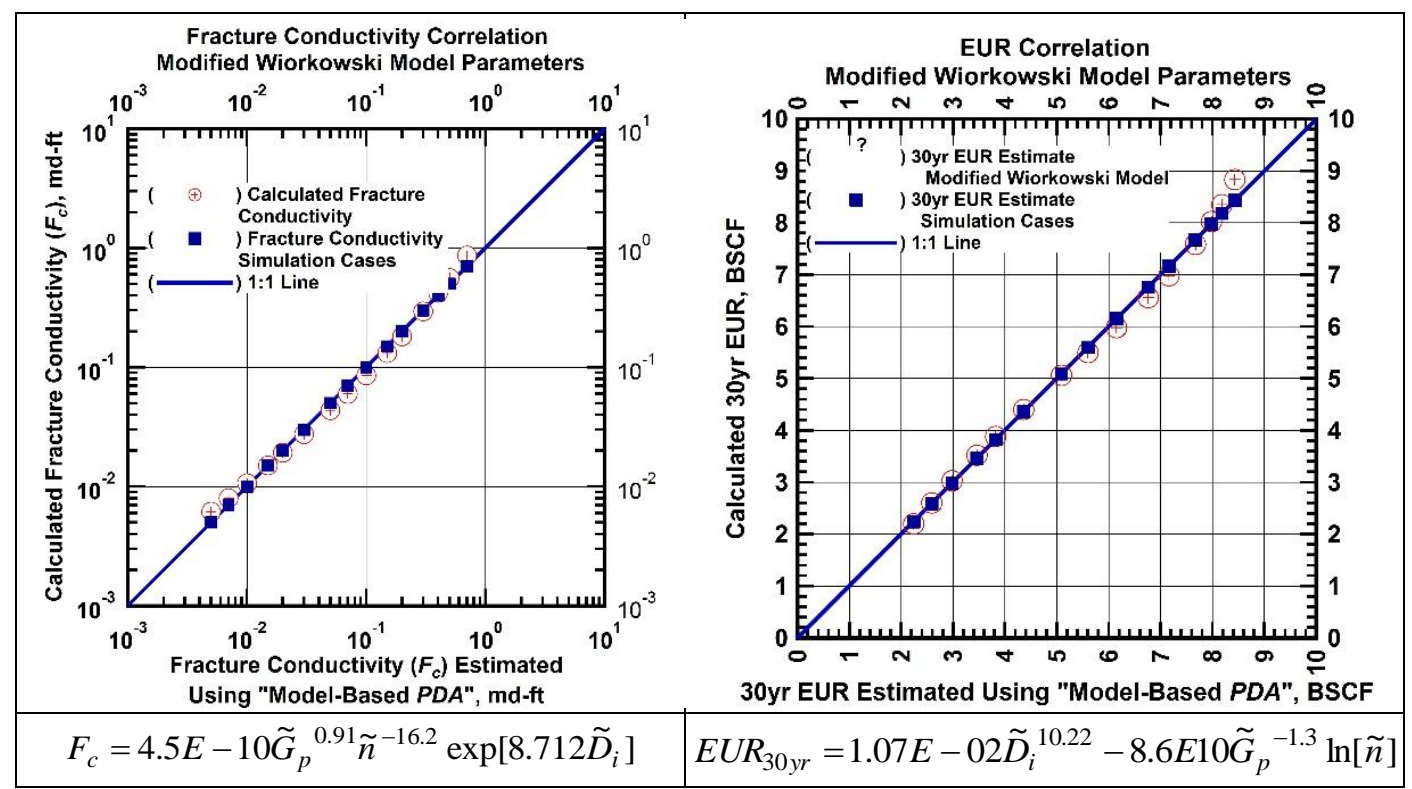

Figure 4.6 - Comparison of fracture conductivity and 30 year EUR values calculated using parametric correlations developed using Modified Wiorkowski model parameters versus numerical simulation models. 


\subsubsection{Modified Ilk Model - Parametric Correlations}

In this section we investigate parametric correlating functions between parameters of Modified Ilk Model and 15 numerical simulation cases with varying fracture conductivities. The gas flowrate $q_{g}, D$ - and $b$ parameter plots are given below in Figs. 4.7a, 4.7b, and 4.8 respectively. The diagnostic functions indicate transient and transition flow regimes followed by boundary-dominated flow in late time part of data.

Fig. 4.7 shows a plot of $b$ - parameter values for various fracture conductivity cases. Fig. 4.7 also shows a dominant transient/transition flow regime characterized by a negative slope straight line decline of $D$ parameter on a log-log plot. We observe a slight deviation of trend and change in slope at around 1,000 days. It should be noted that for all cases Modified Ilk Model $D$ - parameter approaches a constant value at around 20,000 days. This feature exhibits the inherent self-growth limiting feature of Modified Ilk Model that makes this model suitable to match boundary-dominated flow regime. Table 4.3 shows a constant value of zero of parameter $\bar{D}_{\infty}$ for all varying fracture conductivity cases which leads us to its subsequent exclusion from parameter correlation process.

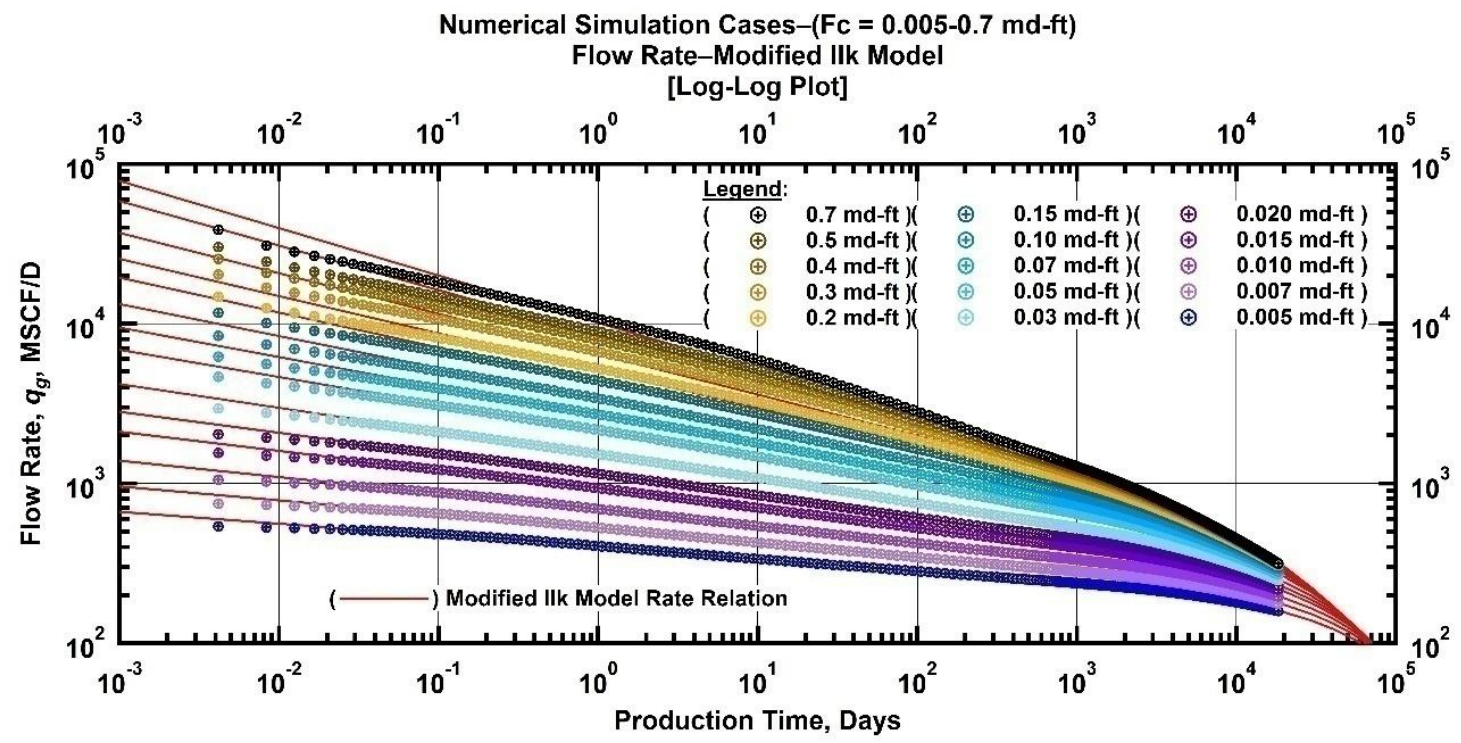

Figure 4.7a - (Log-log Plot): Flow rate $\left(q_{g}\right)$ versus production time. Modified Ilk matches of 15 numerical simulation cases. 


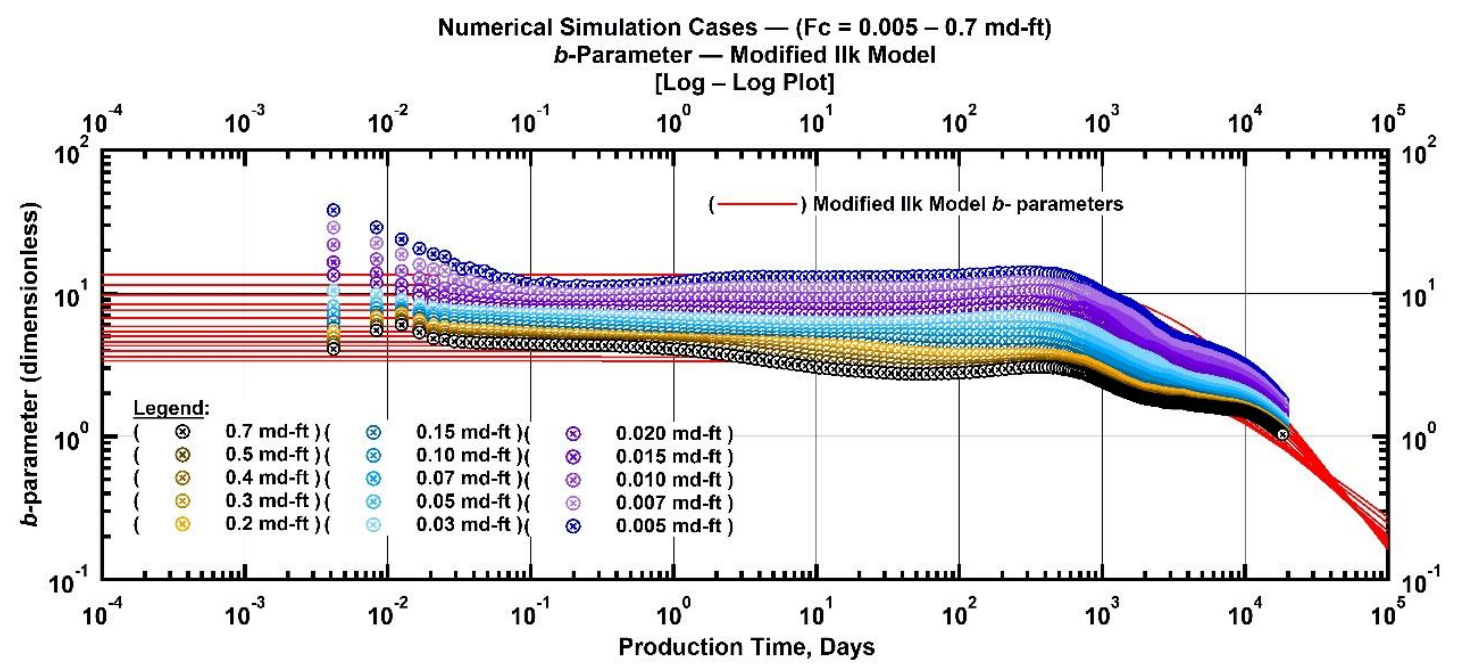

Figure $4.7 \mathrm{~b}-\quad$ (Log-log Plot): $b$-parameter versus production time. Modified Ilk model matches of 15 numerical simulation cases.

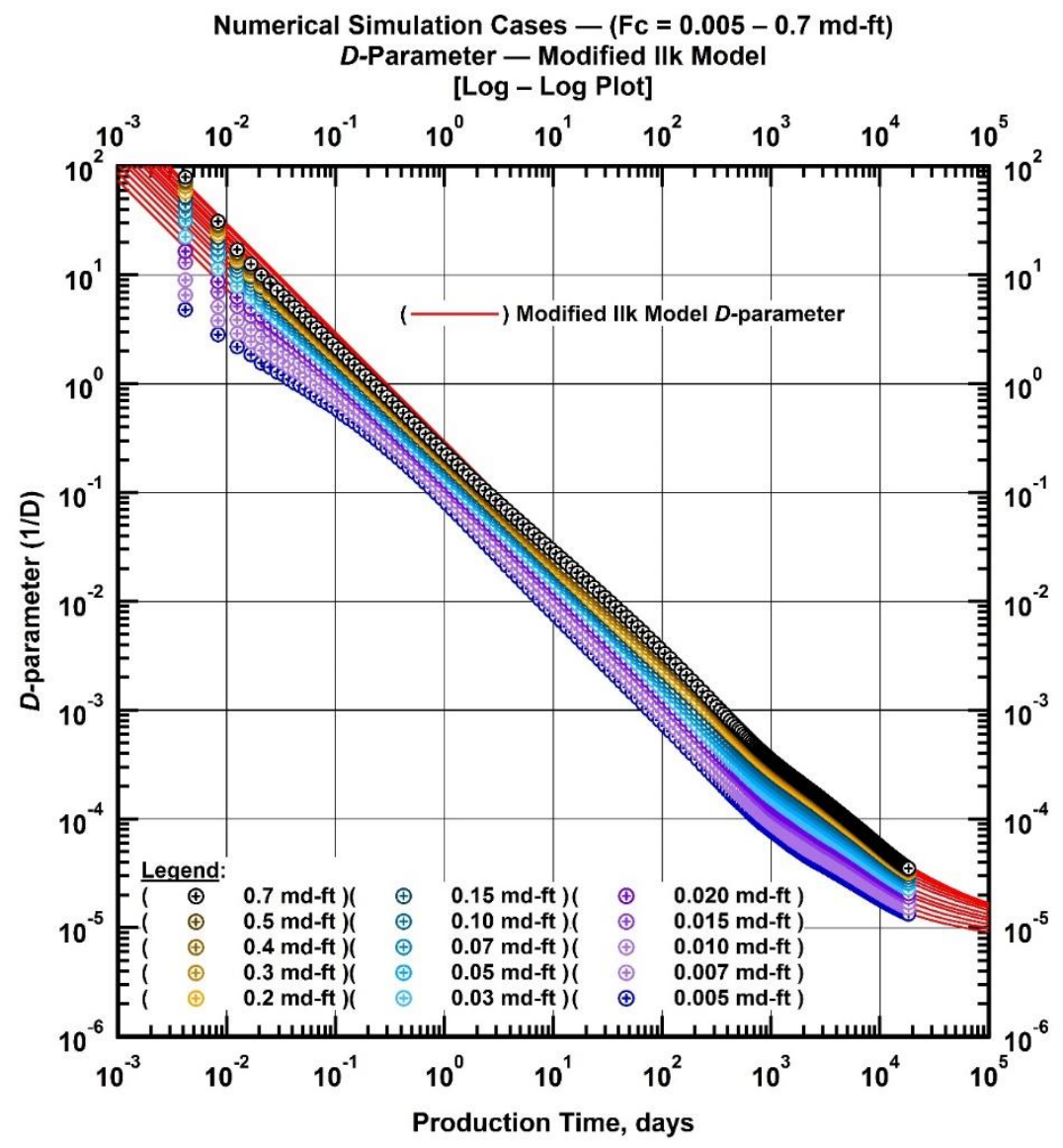

Figure $4.8-(\log -\log$ Plot): $D$-parameter versus production time. Modified Ilk model matches of 15 numerical simulation cases. 
The resulting model parameters are given in Table 4.3.

Table 4.3 - Modified Ilk Model parameters. Model matches to 15 numerical simulation cases.

\begin{tabular}{|c|c|c|c|c|c|c|}
\hline \multicolumn{2}{|c|}{ Num. Sim. Cases } & \multicolumn{5}{|c|}{ Modified Ilk Model Parameters } \\
\hline $\begin{array}{c}F_{c} \\
\text { (md-ft) }\end{array}$ & $\begin{array}{c}\mathrm{G}_{\mathrm{p}, \max (30 \mathrm{yr})} \\
\text { (BSCF) }\end{array}$ & $\begin{array}{c}\bar{G}_{p} \\
(\mathrm{MSCF})\end{array}$ & $\begin{array}{c}\bar{D}_{\infty} \\
\text { (d.less) }\end{array}$ & $\begin{array}{c}\bar{n} \\
\text { (dless) }\end{array}$ & $\begin{array}{c}\bar{D}_{i} \\
\left(\mathrm{D}^{-1}\right)\end{array}$ & $\begin{array}{l}E U R_{M I} \\
\text { (BSCF) }\end{array}$ \\
\hline 0.005 & 2.24 & $2.0 \mathrm{E}+07$ & 0.000 & 0.926 & $2.14 \mathrm{E}-05$ & 2.23 \\
\hline 0.007 & 2.59 & $2.1 \mathrm{E}+07$ & 0.000 & 0.912 & $2.74 \mathrm{E}-05$ & 2.59 \\
\hline 0.010 & 2.98 & $2.1 \mathrm{E}+07$ & 0.000 & 0.897 & $3.56 \mathrm{E}-05$ & 2.983 \\
\hline 0.015 & 3.46 & $2.2 \mathrm{E}+07$ & 0.000 & 0.880 & $4.75 \mathrm{E}-05$ & 3.457 \\
\hline 0.020 & 3.82 & $2.2 \mathrm{E}+07$ & 0.000 & 0.868 & $4.82 \mathrm{E}-05$ & 3.814 \\
\hline 0.030 & 4.36 & $2.3 \mathrm{E}+07$ & 0.000 & 0.851 & 7.73E-05 & 4.346 \\
\hline 0.050 & 4.09 & $2.3 \mathrm{E}+07$ & 0.000 & 0.830 & $1.10 \mathrm{E}-04$ & 4.066 \\
\hline 0.070 & 4.60 & $2.3 \mathrm{E}+07$ & 0.000 & 0.815 & $1.38 \mathrm{E}-04$ & 4.564 \\
\hline 0.100 & 6.15 & $2.4 \mathrm{E}+07$ & 0.000 & 0.800 & $1.75 \mathrm{E}-04$ & 6.092 \\
\hline 0.150 & 6.76 & $2.4 \mathrm{E}+07$ & 0.000 & 0.781 & $2.28 \mathrm{E}-04$ & 6.669 \\
\hline 0.200 & 7.16 & $2.4 \mathrm{E}+07$ & 0.000 & 0.767 & $2.73 \mathrm{E}-04$ & 7.054 \\
\hline 0.300 & 7.67 & $2.5 \mathrm{E}+07$ & 0.000 & 0.747 & $3.48 \mathrm{E}-04$ & 7.546 \\
\hline 0.500 & 8.18 & $2.6 \mathrm{E}+07$ & 0.000 & 0.721 & 4.62E-04 & 8.056 \\
\hline 0.700 & 8.43 & $2.6 \mathrm{E}+07$ & 0.000 & 0.704 & 4.49E-04 & 8.332 \\
\hline
\end{tabular}

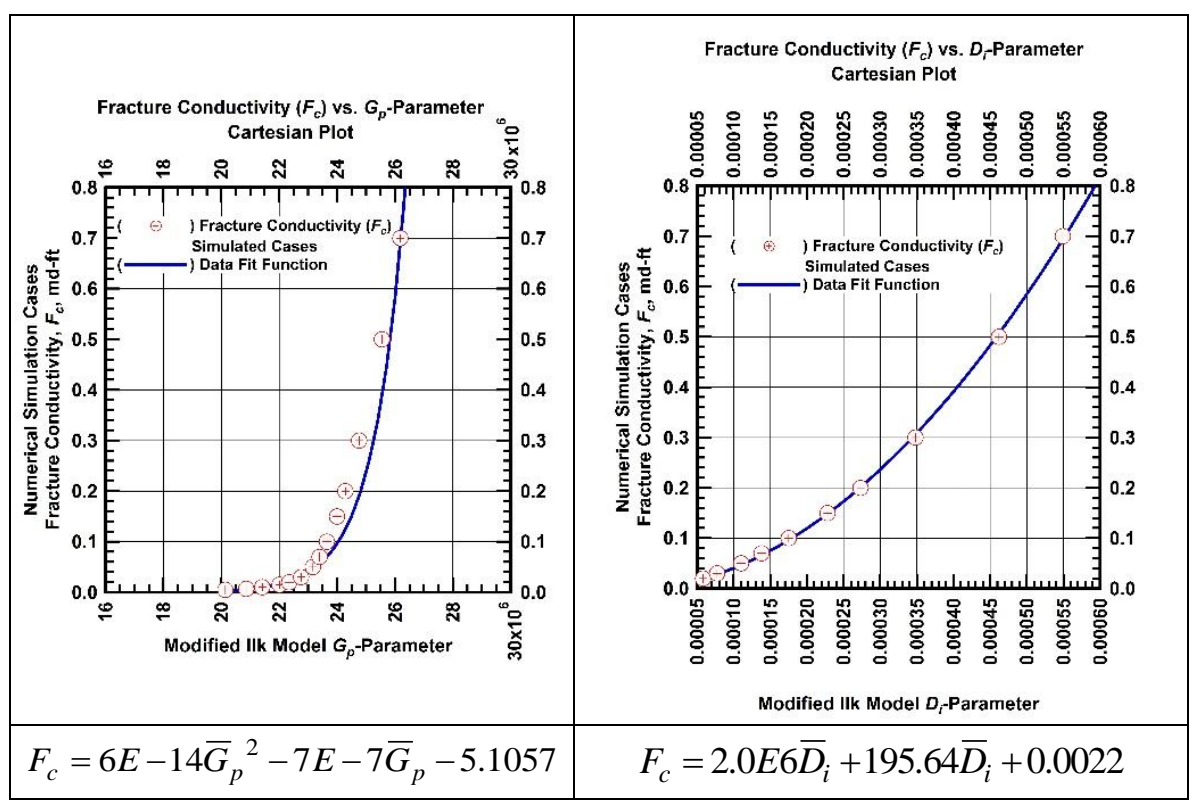




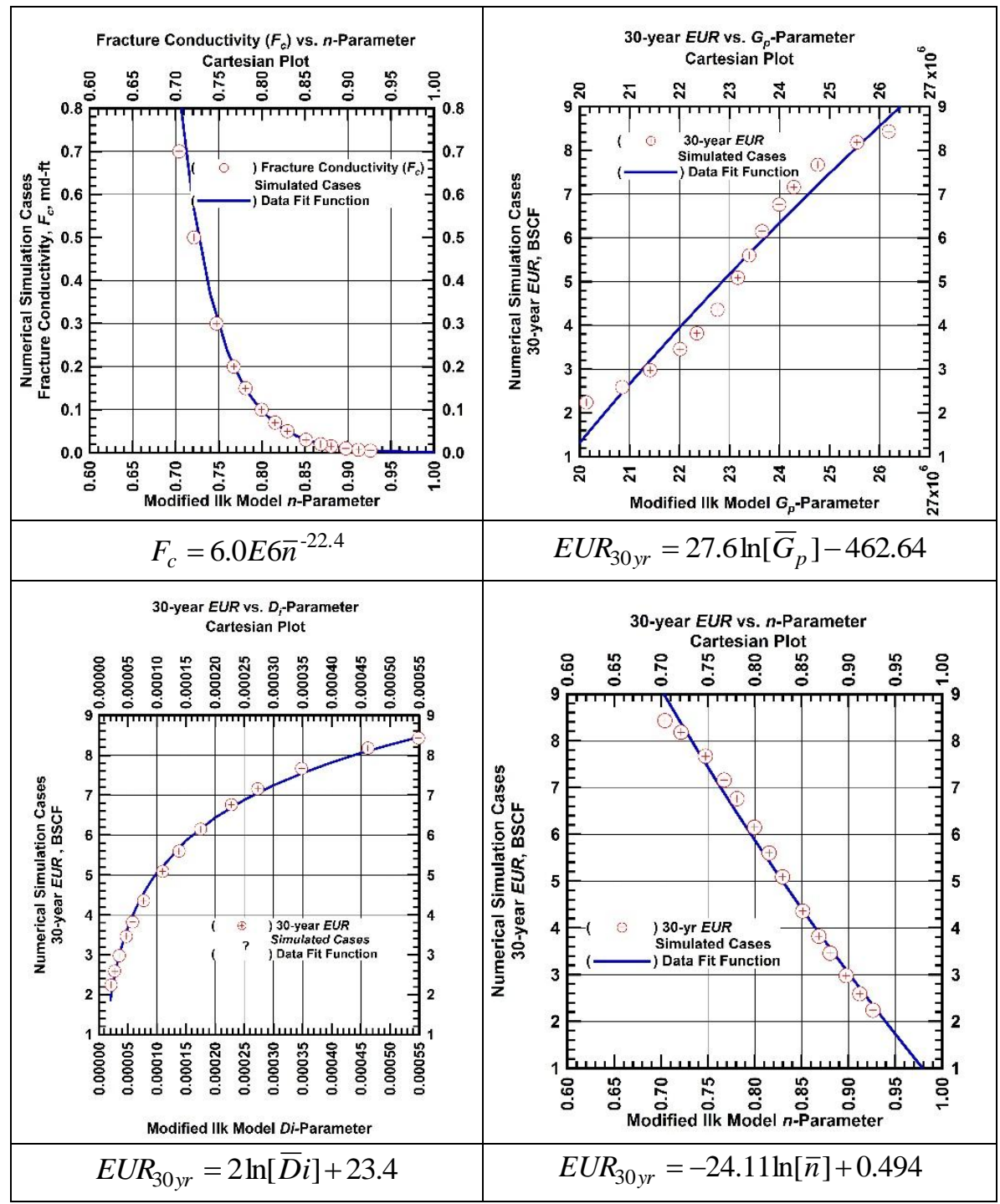

Figure 4.9 - Cross-plots showing relationship between Modified Ilk model parameters and numerical simulation cases fracture conductivity $\left(F_{c}\right)$ and 30 year EUR values.

Fig. 4.9 shows the cross-plots of each considered parameter of Modified Ilk Model parameter for 15 simulation cases against fracture conductivity and 30-year EUR respectively. These plots show the individual correlating relationship of each model parameter with considered reservoir parameters $\left(F_{c}\right.$ and $\left.E U R_{30 y r}\right)$. We also fit a simple parametric function to cross-plot to determine the underlying correlating function for individual parameters. Fig. 4.9 shows the cross-plots along with respective correlating functions.

Finally, we relate reservoir parameters with Modified Ilk model parameters by proposing parametric correlations based on the correlating functions we identified in Fig. 4.9. Fig. 4.9 shows that fracture 
conductivity can be correlated to model parameters by a combination of exponential and second degree polynomial functions. We propose the correlating function as:

$$
F_{c}=\exp \left[a_{01} \bar{G}_{p}\right] \exp \left[a_{02} \bar{n}\right]+a_{03} \bar{D}_{i}^{2}+a_{04} \bar{D}_{i}
$$

Here, $a_{01}, a_{02}, a_{03}$ and $a_{04}$ are coefficients to be determined through least square regression. Similarly we propose the following correlating function to estimate the 30 year $E U R\left(E U R_{30 y r}\right)$.

$$
E U R_{30 y r}=a_{01} \widetilde{D}_{i}^{a_{02}}-a_{03} \widetilde{G}_{p}^{a_{04}} \ln [\tilde{n}]
$$

Fig. 4.10 shows the resulting model fits for proposed parametric correlations. It can be observed that the proposed correlating functions provide a reliable estimate of the reservoir properties $\left(F_{c}\right.$ and $\left.E U R_{30} y r\right)$ for the case of a well within the same reservoir system with similar completion and production constraints.

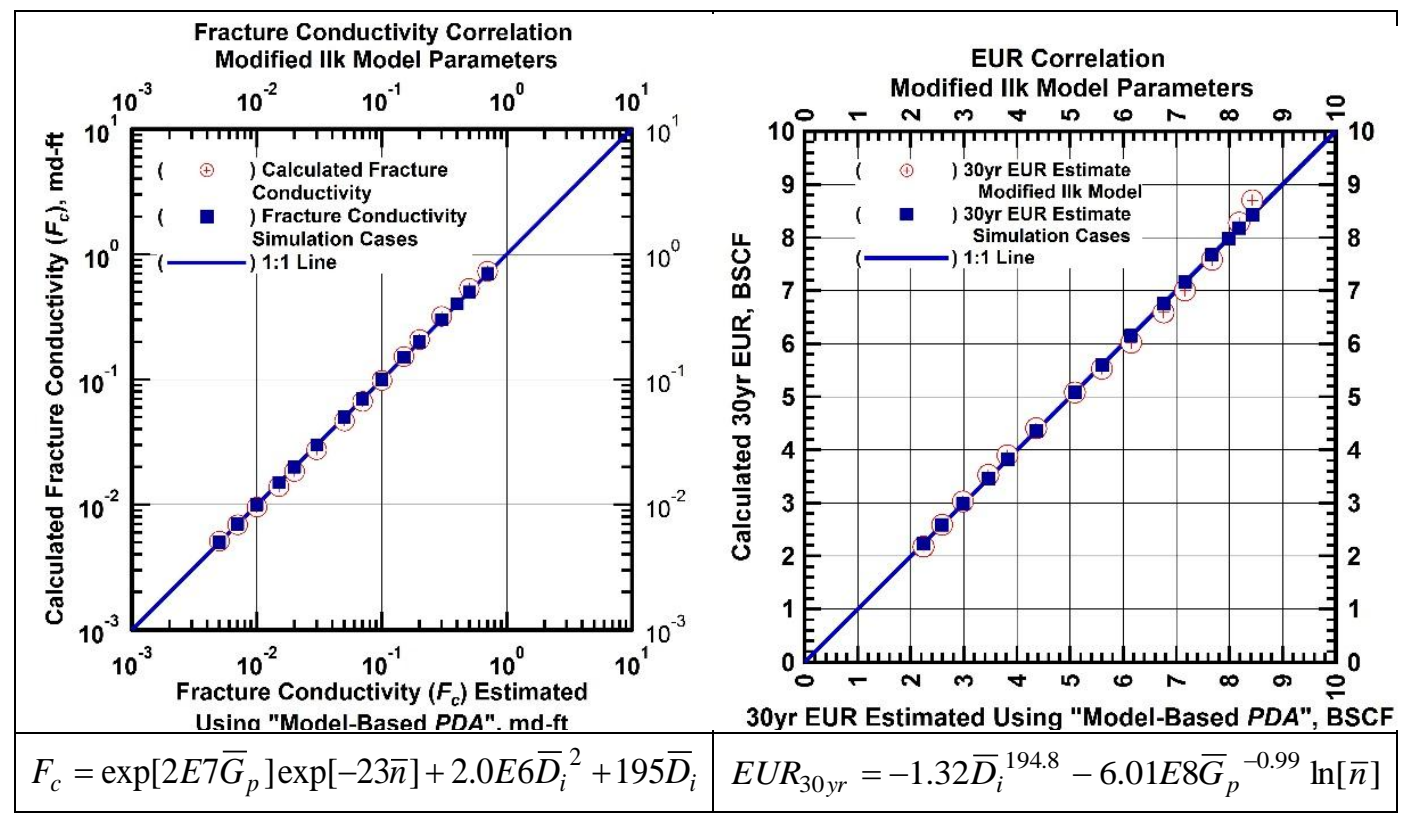

Figure 4.10 - Comparison of fracture conductivity and 30 year EUR values calculated using parametric correlations developed using Modified Ilk model parameters versus numerical simulation models. 


\subsection{Fracture Half Length - Parametric Correlations}

In this section we will investigate the parametric correlation between two proposed models and fracture half-length for 15 numerical simulation cases with fracture half-length varying between 50 and $400 \mathrm{ft}$. The model input parameters for numerical simulation case are given below.

Table 4.4 - Reservoir and fluid properties for numerical simulation case (horizontal well with multiple transverse fractures) with varying fracture half-lengths

$\begin{array}{lll}\text { Reservoir Properties } & & \\ \quad \text { Net pay thickness, } h & & 160 \mathrm{ft} \\ \quad \text { Formation permeability, } k & = & 0.5 \mu \mathrm{D} \\ \text { Fracture conductivity } & = & 0.005 \mathrm{md}-\mathrm{ft} \\ \text { Wellbore Radius, } r_{w} & = & 0.1 \mathrm{ft} \\ \quad \text { Formation compressibility, } c_{f} & = & 3 \times 10^{-6} \mathrm{psi}^{-1} \\ \quad \text { Porosity, } \phi & = & 0.05(\text { fraction }) \\ \text { Initial reservoir pressure, } p_{i} & = & 5000 \mathrm{psi} \\ \quad \text { Gas saturation, } s_{g} & = & 1.0 \text { fraction } \\ \quad \text { Skin factor, } s & = & -5(\text { dimensionless }) \\ \text { Reservoir temperature, } T_{r} & = & 212^{\circ} \mathrm{F} \\ \text { Fluid properties: } & & \\ \quad \text { Gas specific gravity, } \gamma_{g} & = & 0.7(\text { air }=1) \\ \text { Hydraulically fractured well model } & \text { parameters: } \\ \text { Fracture half-length, } x_{f} & = & 50-400 \mathrm{ft} \\ \quad \text { Number of fractures } & = & 15 \\ \text { Horizontal well length } & = & 6561.7 \mathrm{ft} \\ \text { Production parameters: } & & \\ \text { Flowing pressure, } p_{w f} & = & 500 \mathrm{psia} \\ \text { Production time, } t & = & 10,950 \text { days }(\sim 30 \text { years })\end{array}$

In the following two sections we present parametric correlations for both models respectively.

\subsubsection{Modified Wiorkowski Model - Parametric Correlations}

We investigate parametric correlating functions between parameters of Modified Wiorkowski Model and 15 numerical simulation cases with varying fracture half-lengths. The gas flowrate $q_{g}, D$ - and $b$-parameter plots are given below in Figs. 4.11, 4.12, and 4.13 respectively. The diagnostic functions indicate transient and transition flow regimes followed by boundary-dominated flow in late time part of data.

Fig. 4.13 shows a plot of $b$ - parameter values for various fracture conductivity cases. We observe a slight deviation of trend and change in slope at around 1,000 days. It should be noted that for all cases Modified Wiorkowski Model $D$ - parameter approaches a constant value at around 20,000 days. 
For the case of varying fracture half-lengths it can be readily observed that we have a much lesser spread for all parameters. Table 4.6 shows a constant value of unity of parameter $\tilde{a}$ for all varying fracture conductivity cases which leads us to its subsequent exclusion from parameter correlation process.

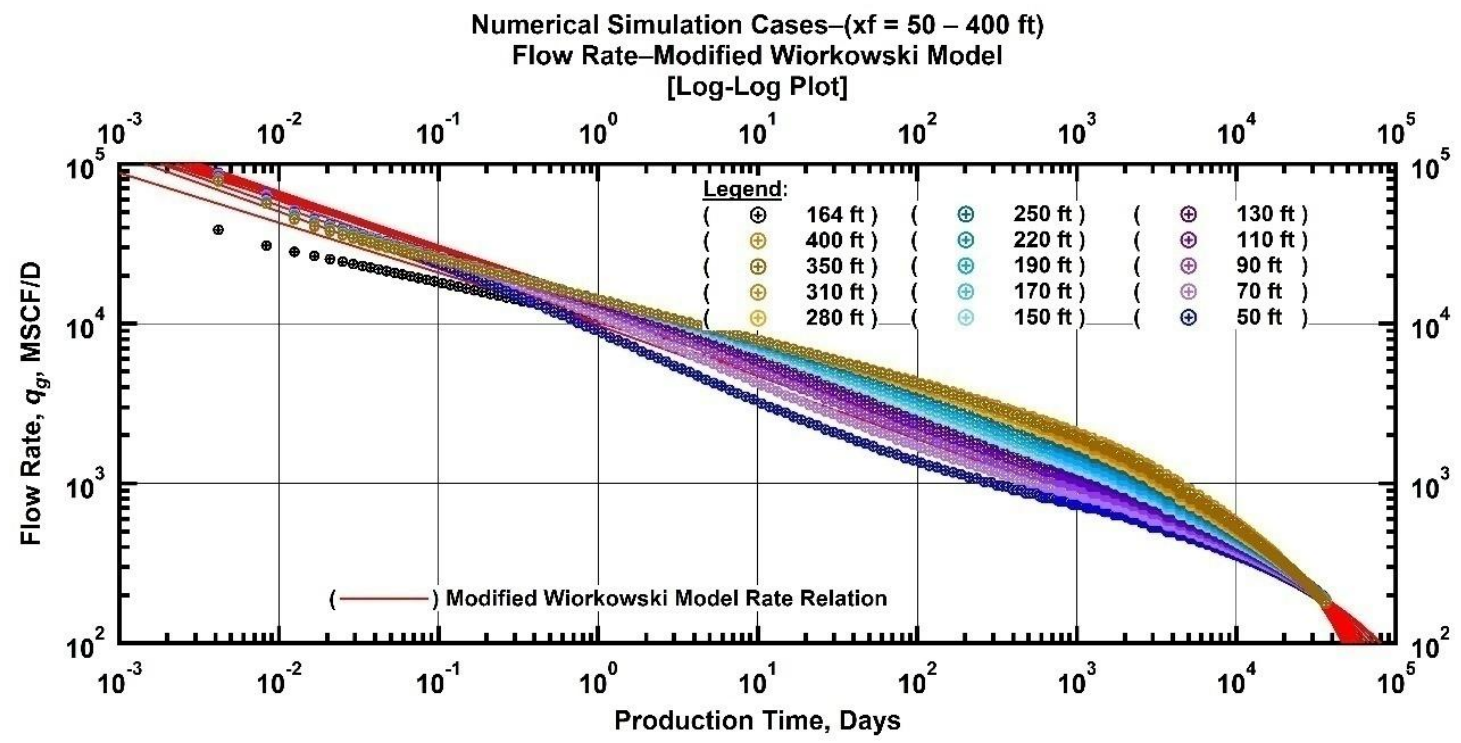

Figure $4.11-\quad$ (Log-log Plot): Flow rate $\left(q_{g}\right)$ versus production time. Modified Wiorkowski model matches of 15 numerical simulation cases with varying fracture halflengths.

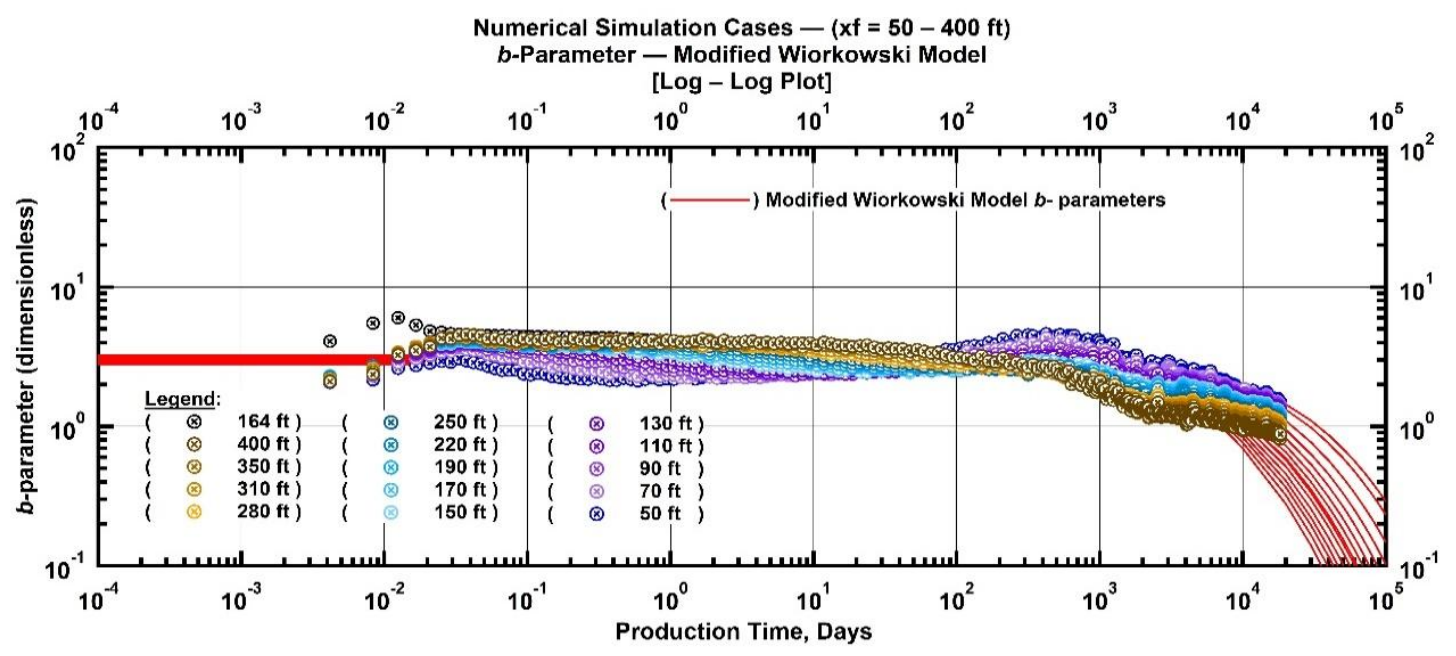

Figure $4.12-\quad(\log -\log$ Plot): $b$-parameter versus production time. MWM model matches of 15 numerical simulation cases with varying fracture half-lengths, 


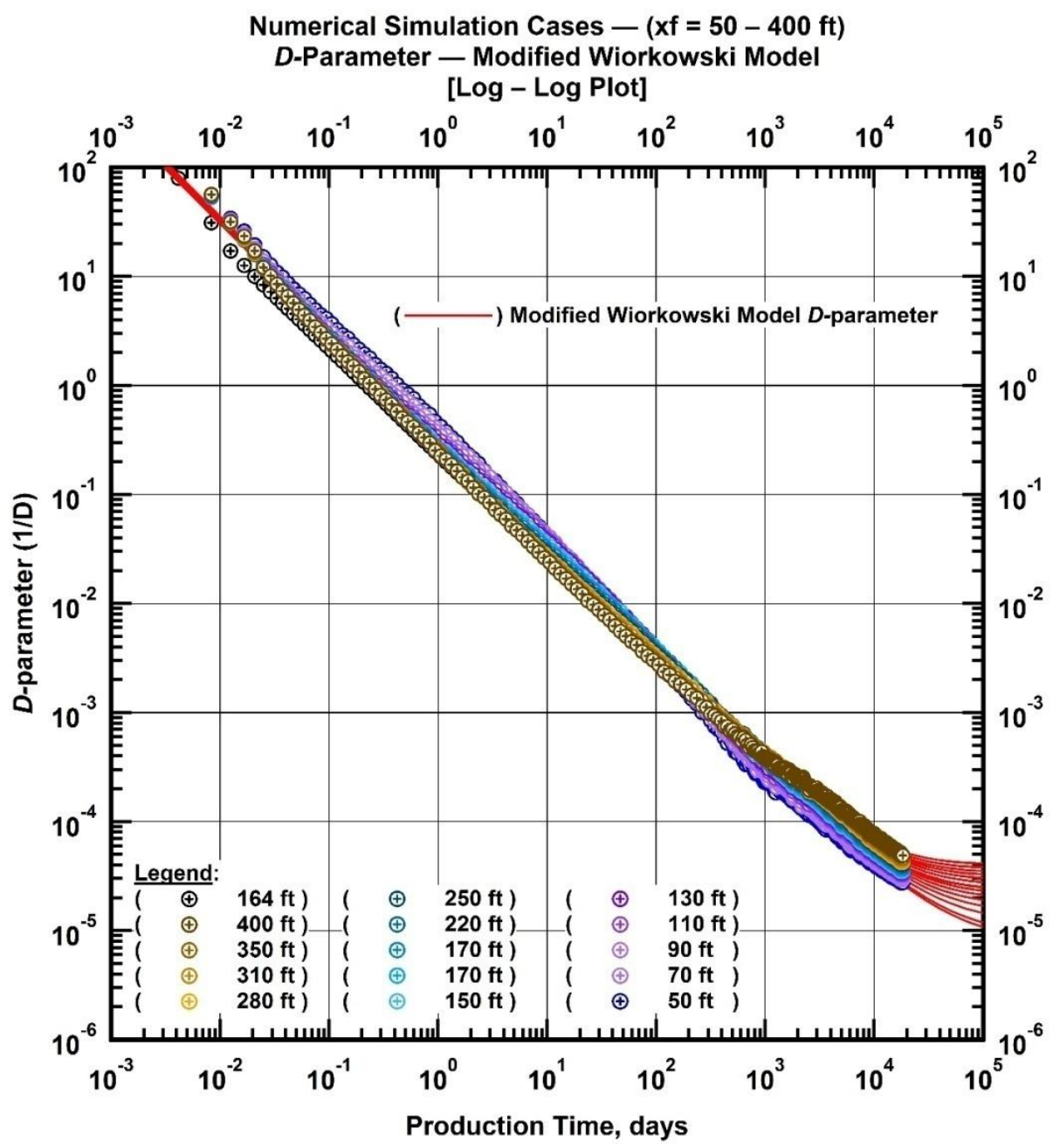

Figure $4.13-\quad$ (Log-log Plot): $D$-parameter versus production time. MWM model matches of 15 numerical simulation cases with varying fracture half-lengths.

Table 4.5 shows the matching model parameters. 
Table 4.5 - Modified Wiorkowski Model parameters. Model matches to 15 numerical simulation cases with varying fracture half-lengths.

Num. Sim. Cases

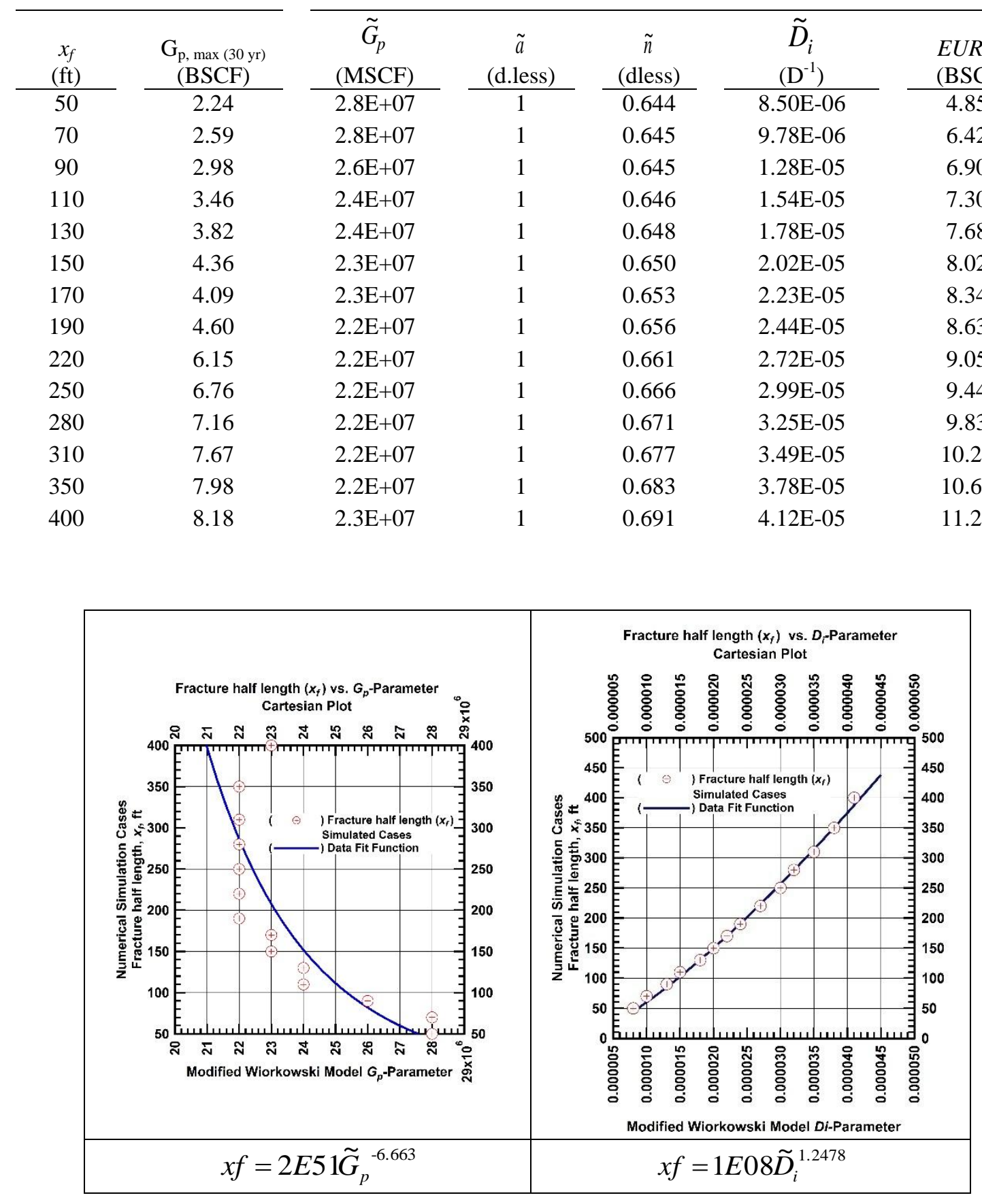




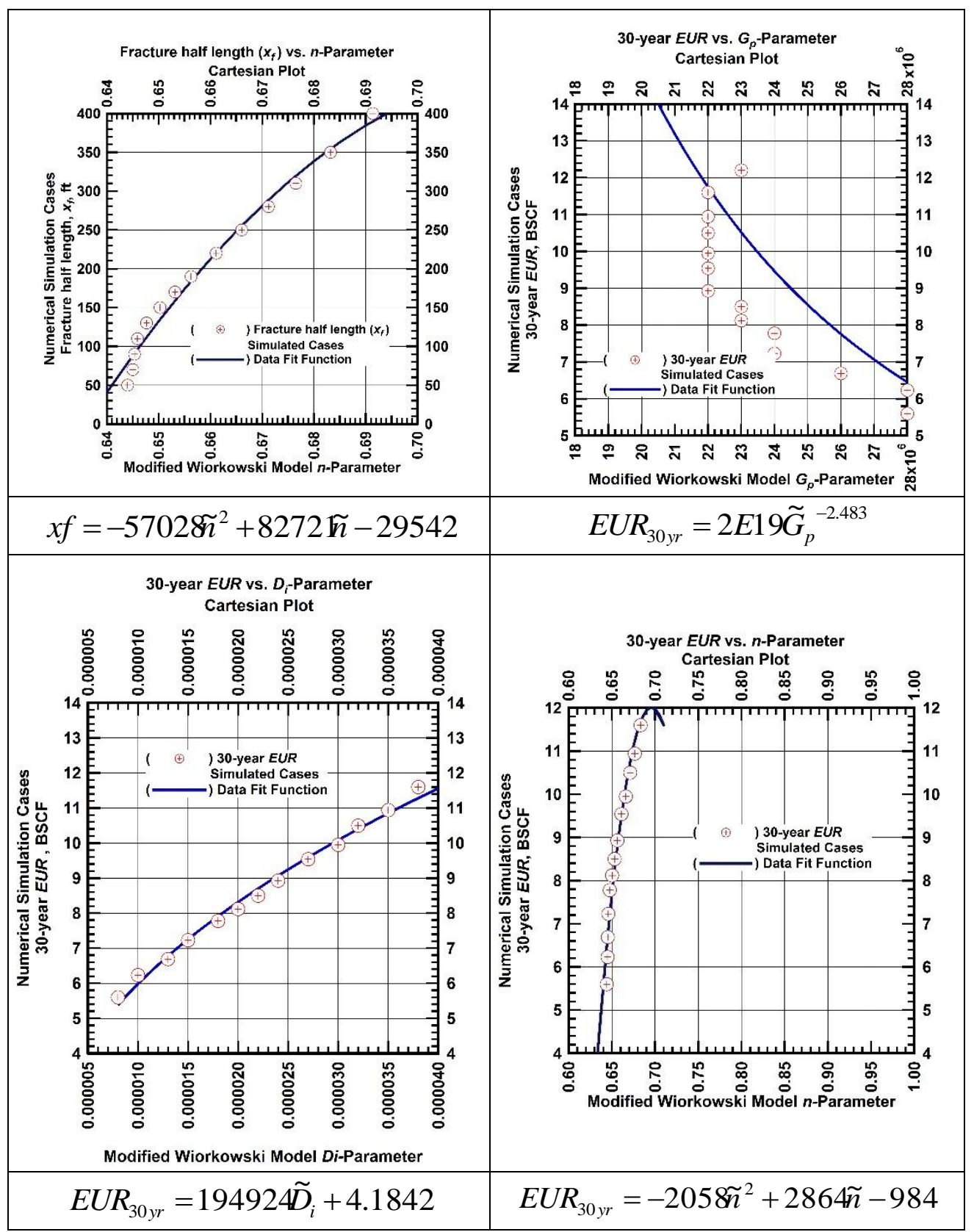

Figure 4.14 - Cross-plots showing relationship between Modified Wiorkowski model parameters and numerical simulation case fracture half-length $(x f)$ and 30 year EUR estimates.

Fig. 4.14 shows the cross-plots of each considered parameter of Modified Wiorkowski Model parameter for 15 simulation cases against fracture half-length and 30-year EUR respectively. We also fit a simple parametric function to cross-plot to determine the underlying correlating function for individual parameters. Fig. 4.14 shows the cross-plots along with respective correlating functions. 
Fig. 4.14 shows that fracture half-length can be correlated to model parameters by a combination of power and second degree polynomial functions. We propose the correlating function as:

$$
x f=a_{01} \tilde{D}_{i}^{a_{02}}\left(\tilde{n}^{2 a_{03}}-a_{03} \tilde{n}\right)
$$

Here, $a_{01}, a_{02}$ and $a_{03}$ are coefficients to be determined through least square regression. Similarly we propose the following correlating function to estimate the 30 year $E U R\left(E U R_{30 y r}\right)$.

$$
E U R_{30 y r}=a_{01} \tilde{D}_{i}+a_{02}\left(a_{03} \tilde{n}^{2}-a_{04} \tilde{n}\right)
$$

Fig. 4.15 shows the resulting model fits for proposed parametric correlations. It can be observed that the proposed correlating functions provide a reliable estimate of the reservoir properties ( $x f$ and $\left.E U R_{30} y r\right)$ for the case of a well within the same reservoir system with similar completion and production constraints.

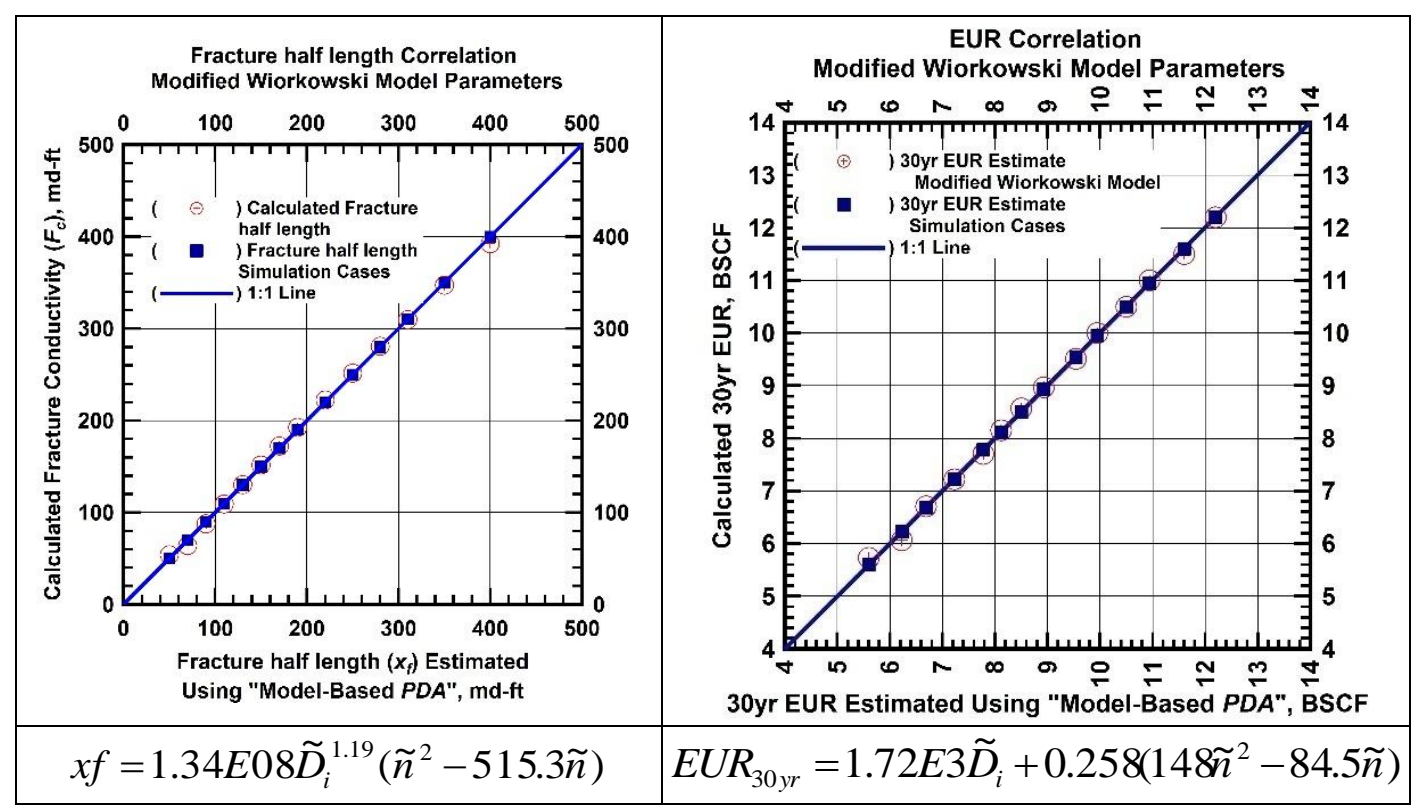

Figure 4.15 - Comparison of fracture conductivity and 30 year EUR values calculated using parametric correlations developed using Modified Wiorkowski model parameters versus numerical simulation models. 


\subsubsection{Modified Ilk Model - Parametric Correlations}

In this section we investigate parametric correlating functions between parameters of Modified Ilk Model and 15 numerical simulation cases with varying fracture lengths. The gas flowrate $q_{g}, D$ - and $b$-parameter plots are given below in Figs. 4.16, 4.17, and 4.18 respectively. The diagnostic functions indicate transient and transition flow regimes followed by boundary-dominated flow in late time part of data.

Fig. 4.17 shows a plot of $b$ - parameter values for various fracture conductivity cases. It should be noted that for all cases Modified Ilk Model $D$ - parameter approaches a constant value at around 20,000 days. For the case of varying fracture half-lengths it can be readily observed that we have a much lesser spread for all parameters.

Table 4.6 shows a constant value of zero of parameter $\bar{D}_{\infty}$ for all varying fracture conductivity cases which leads us to its subsequent exclusion from parameter correlation process.

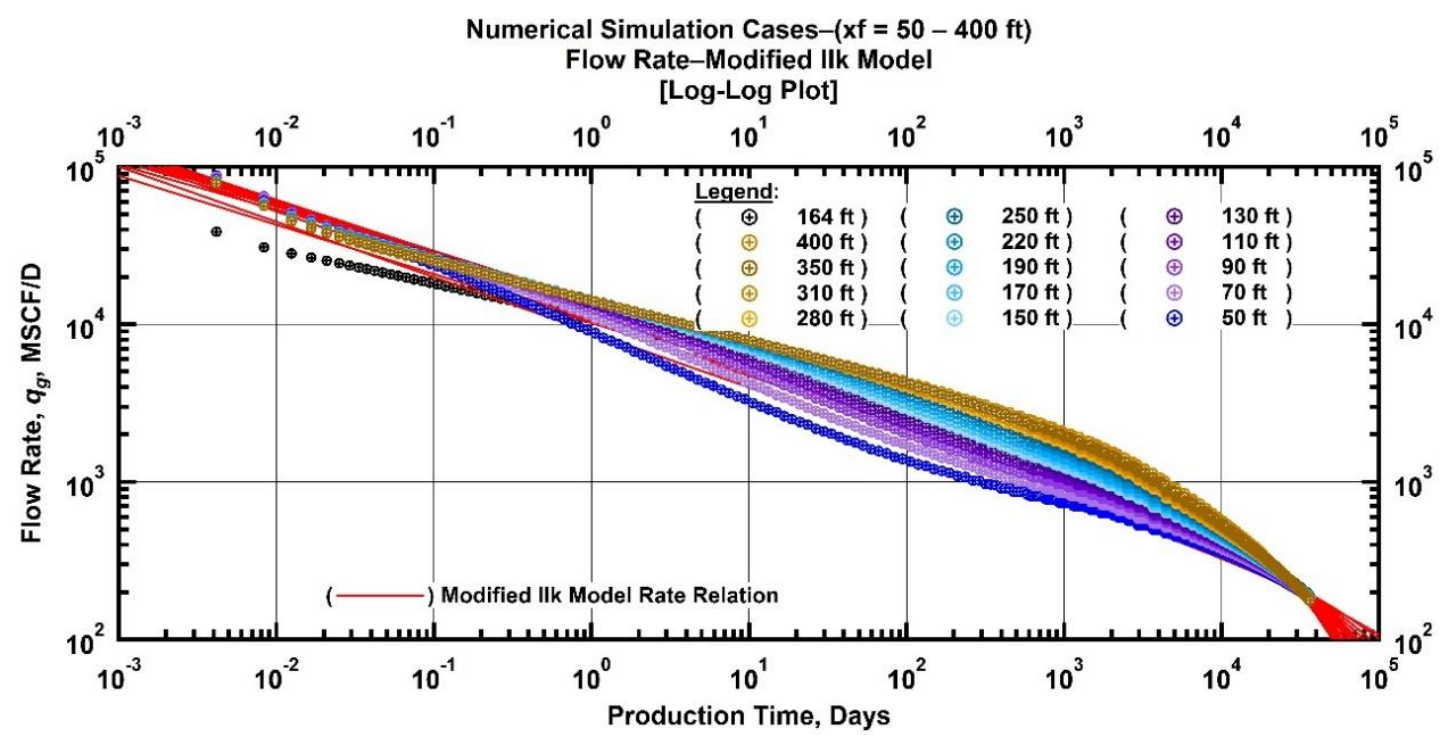

Figure 4.16 - (Log-log Plot): Flow rate $\left(q_{g}\right)$ versus production time. Modified Ilk matches of 15 numerical simulation cases with varying fracture half-lengths. 


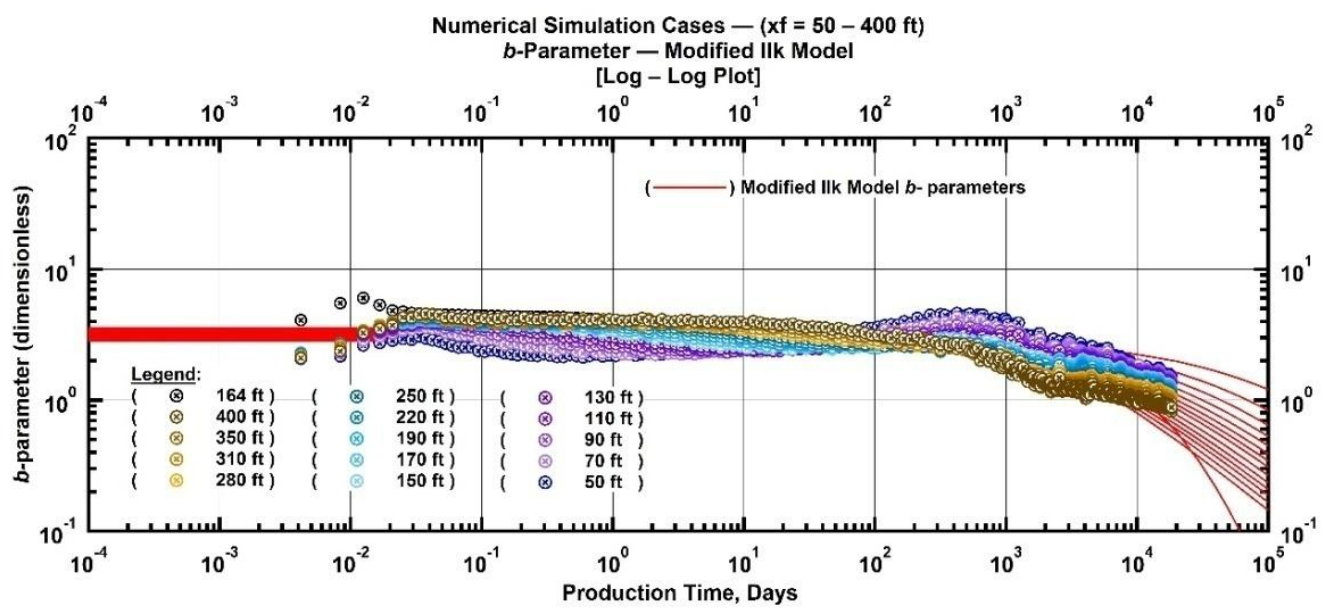

Figure $4.17-\quad$ (Log-log Plot): $b$-parameter versus production time. Modified Ilk model matches of 15 numerical simulation cases with varying fracture half-lengths.

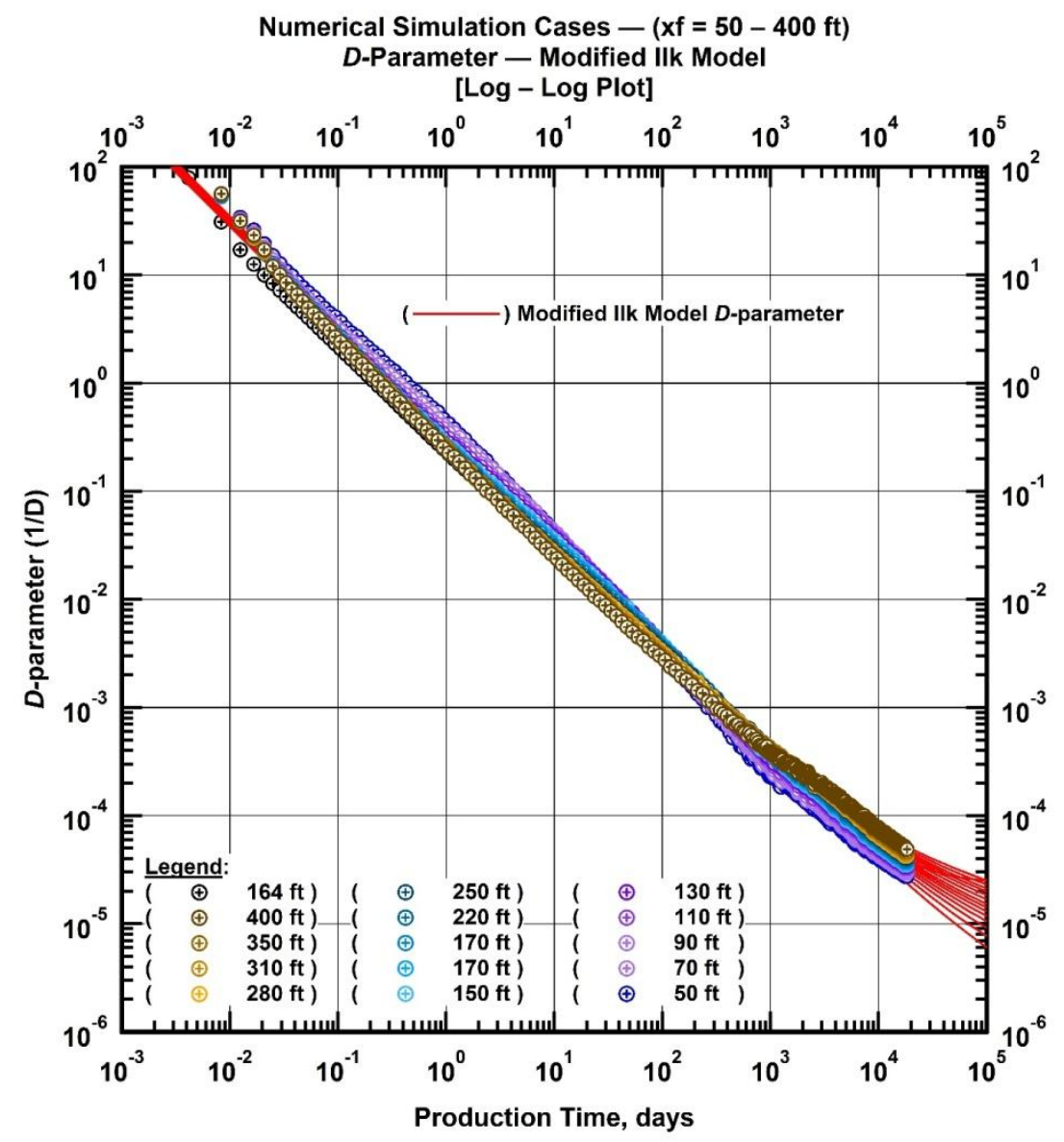

Figure $4.18-\quad(\log -\log$ Plot): $D$ - parameter versus production time. Modified Ilk model matches of 15 numerical simulation cases with varying fracture half-lengths. 
The resulting model parameters are given in Table 4.6.

Table $4.6-$ Modified Ilk Model parameters. Model matches to 15 numerical simulation cases with varying fracture half-lengths.

\begin{tabular}{|c|c|c|c|c|c|c|}
\hline \multicolumn{2}{|c|}{ Num. Sim. Cases } & \multicolumn{5}{|c|}{ Modified Ilk Model Parameters } \\
\hline $\begin{array}{c}x_{f} \\
(\mathrm{ft})\end{array}$ & $\begin{array}{c}\mathrm{G}_{\mathrm{p}, \max (30 \mathrm{yr})} \\
\text { (BSCF) }\end{array}$ & $\begin{array}{c}\bar{G}_{p} \\
(\mathrm{MSCF})\end{array}$ & $\begin{array}{c}\bar{D}_{\infty} \\
\text { (d.less) }\end{array}$ & $\begin{array}{c}\bar{n} \\
\text { (dless) }\end{array}$ & $\begin{array}{c}\bar{D}_{i} \\
\left(\mathrm{D}^{-1}\right)\end{array}$ & $\begin{array}{c}E U R_{M I} \\
\text { (BSCF) }\end{array}$ \\
\hline 50 & 4.60 & $6.2 \mathrm{E}+07$ & 0.000 & 0.650 & $2.21 \mathrm{E}-04$ & 4.82 \\
\hline 70 & 6.23 & $4.5 \mathrm{E}+07$ & 0.000 & 0.652 & $3.54 \mathrm{E}-04$ & 6.44 \\
\hline 90 & 6.69 & $3.7 \mathrm{E}+07$ & 0.000 & 0.654 & $4.71 \mathrm{E}-04$ & 6.970 \\
\hline 110 & 7.23 & $3.3 \mathrm{E}+07$ & 0.000 & 0.657 & $4.60 \mathrm{E}-04$ & 7.420 \\
\hline 130 & 7.78 & $3.1 \mathrm{E}+07$ & 0.000 & 0.661 & $6.29 \mathrm{E}-04$ & 7.830 \\
\hline 150 & 8.12 & $2.9 \mathrm{E}+07$ & 0.000 & 0.665 & $6.83 \mathrm{E}-04$ & 8.200 \\
\hline 170 & 8.50 & $2.8 \mathrm{E}+07$ & 0.000 & 0.670 & $7.24 \mathrm{E}-04$ & 8.550 \\
\hline 190 & 8.93 & $2.7 \mathrm{E}+07$ & 0.000 & 0.674 & $7.56 \mathrm{E}-04$ & 8.880 \\
\hline 220 & 9.54 & $2.6 \mathrm{E}+07$ & 0.000 & 0.681 & 7.89E-04 & 9.340 \\
\hline 250 & 9.95 & $2.5 \mathrm{E}+07$ & 0.000 & 0.687 & $8.11 \mathrm{E}-04$ & 9.770 \\
\hline 280 & 10.50 & $2.5 \mathrm{E}+07$ & 0.000 & 0.694 & $8.22 \mathrm{E}-04$ & 10.200 \\
\hline 310 & 10.94 & $2.5 \mathrm{E}+07$ & 0.000 & 0.700 & $8.26 \mathrm{E}-04$ & 10.600 \\
\hline 350 & 11.60 & $2.5 \mathrm{E}+07$ & 0.000 & 0.707 & $8.25 \mathrm{E}-04$ & 11.100 \\
\hline 400 & 12.20 & $2.5 \mathrm{E}+07$ & 0.000 & 0.717 & 8.13E-04 & 11.700 \\
\hline
\end{tabular}

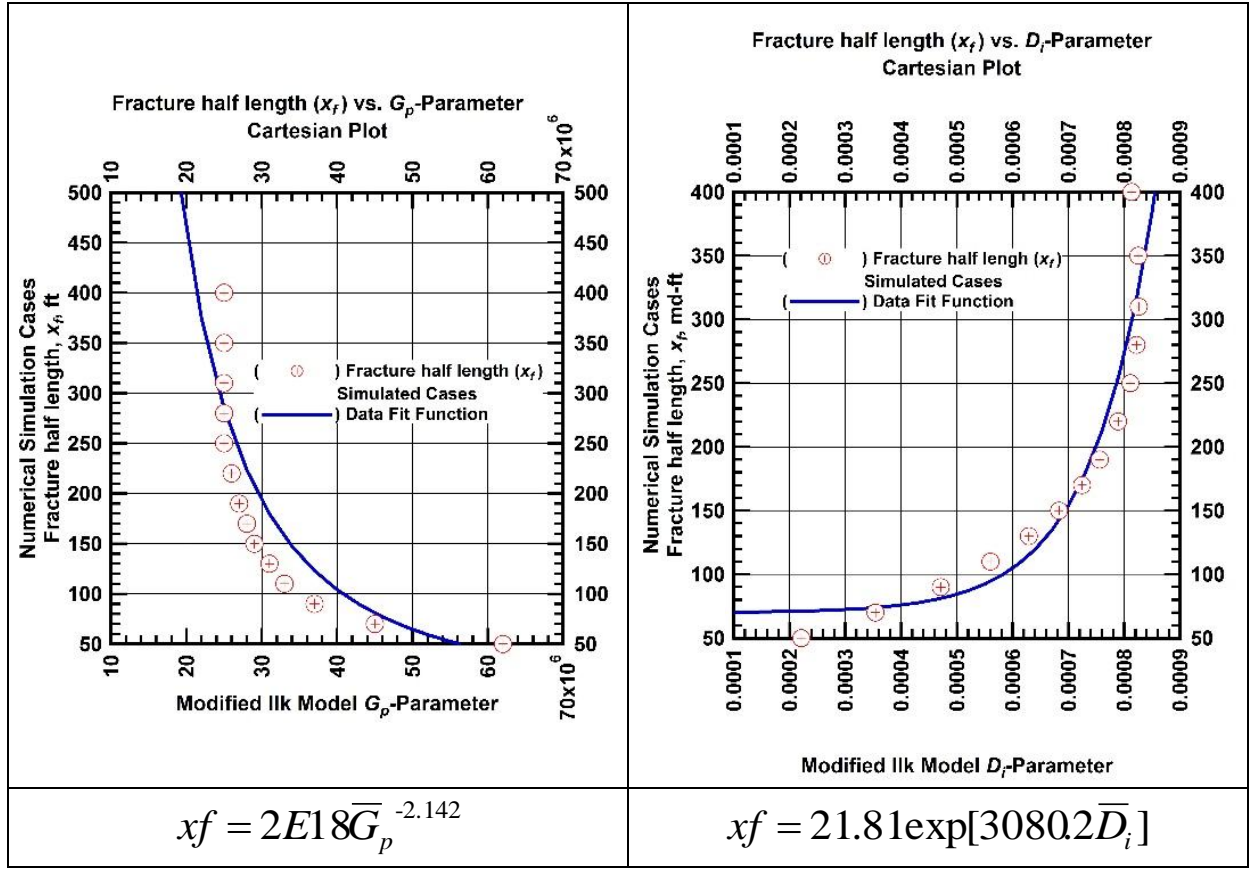




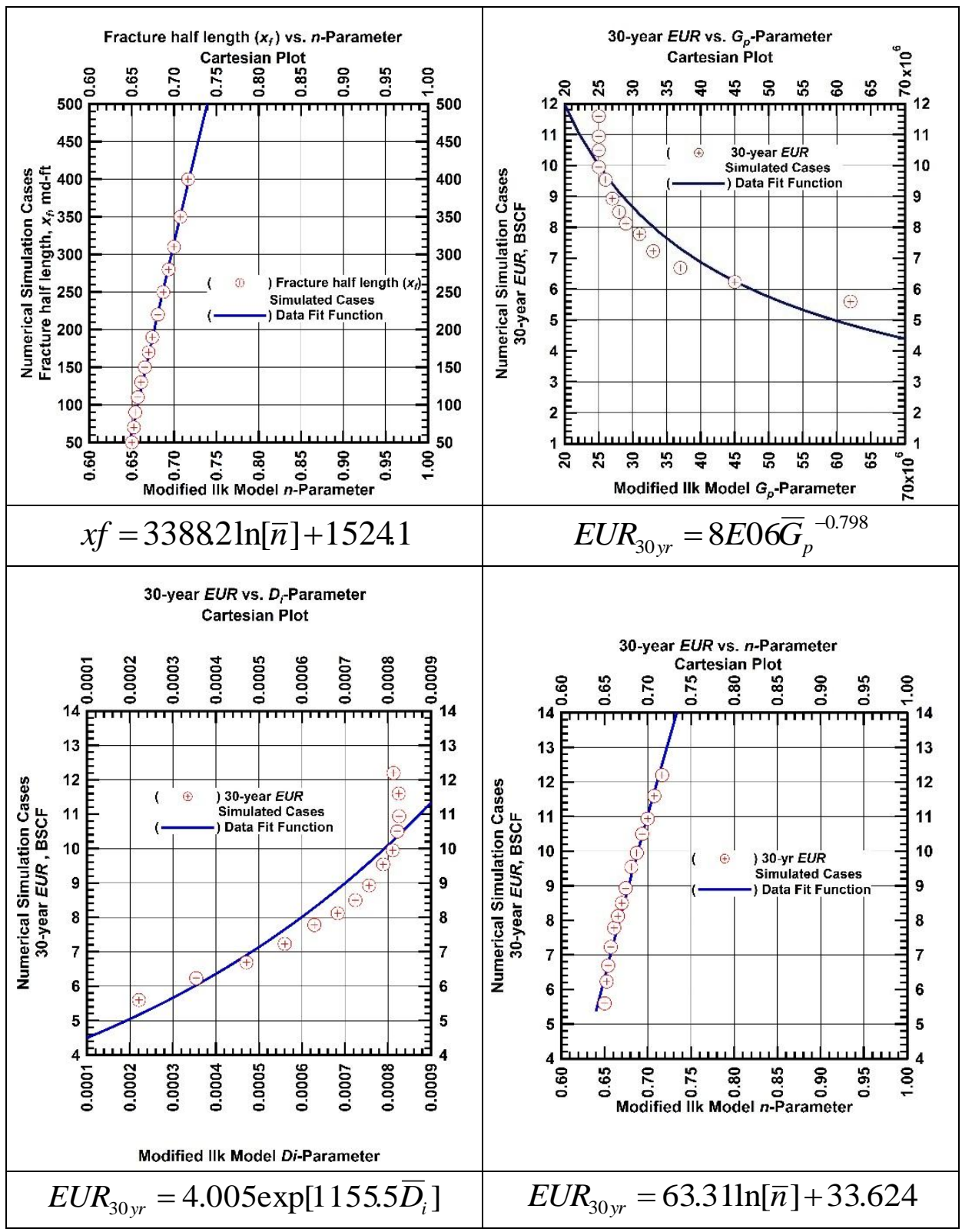

Figure 4.19- Cross-plots showing relationship between Modified Ilk model parameters and numerical simulation cases fracture half-length $(x f)$ and 30 year $E U R$ values.

Fig. 4.19 shows the cross-plots of each considered parameter of Modified Ilk Model parameter for 15 simulation cases against fracture conductivity and 30-year EUR respectively. We also fit a simple parametric function to cross-plot to determine the underlying correlating function for individual parameters. Fig. 4.19 shows the cross-plots along with respective correlating functions. 
Fig. 4.19 shows that fracture half-length can be correlated to model parameters by a combination of exponential and logarithmic functions. We propose the correlating function as:

$$
x f=a_{01} \ln [\bar{n}]+a_{02} \exp \left[\bar{D}_{i}\right]
$$

Here, $a_{01}$ and $a_{02}$ are coefficients to be determined through least square regression. Similarly we propose the following correlating function to estimate the 30 year $E U R\left(E U R_{30 y r}\right)$.

$$
E U R_{30 y r}=a_{01} \ln \left[a_{02} \bar{n}\right] \exp \left[a_{03} \bar{D}_{i}\right]
$$

Fig. 4.20 shows the resulting model fits for proposed parametric correlations. This indicates that these correlating functions can provide reasonable estimates of the reservoir properties ( $x f$ and $\left.E U R_{30} y r\right)$ for the case of a well within the same reservoir system with similar completion and production constraints.

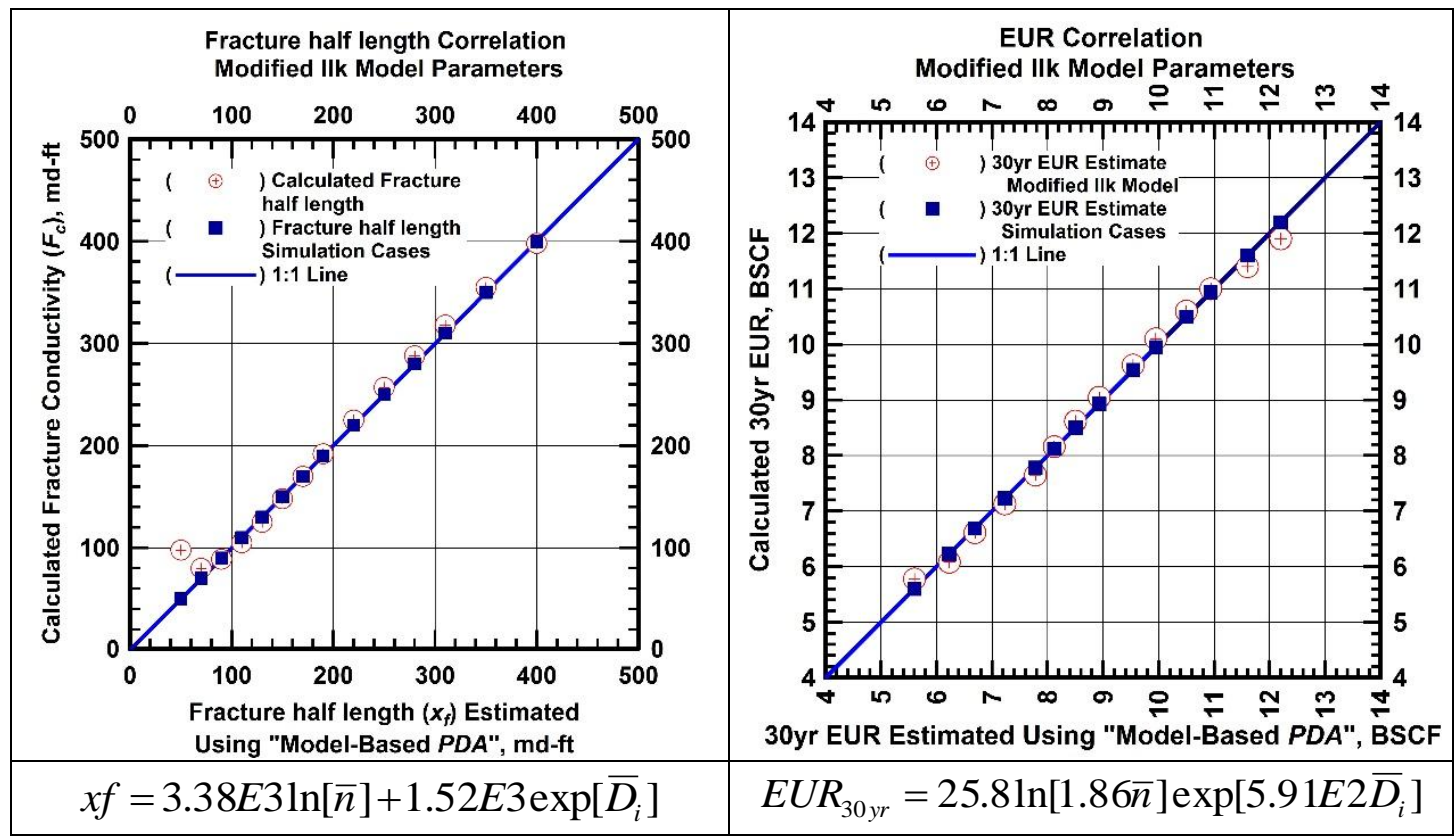

Figure 4.20 - Comparison of fracture conductivity and 30 year EUR values calculated using parametric correlations developed using Modified Ilk model parameters versus numerical simulation models. 


\subsection{Formation Permeability - Parametric Correlations}

In this section we will investigate the parametric correlation between two proposed models and permeability for 15 numerical simulation cases with formation permeability varying between $0.0001-0.005$ md. The model input parameters for numerical simulation case are given below.

Table $4.7 \quad$ Reservoir and fluid properties for numerical simulation case (horizontal well with multiple transverse fractures) with varying formation permeability.

$\begin{array}{lll}\text { Reservoir Properties } & & \\ \quad \text { Net pay thickness, } h & & 160 \mathrm{ft} \\ \quad \text { Formation permeability, } k & = & 0.0005-0.005 \mathrm{md} \\ \text { Fracture conductivity } & = & \text { infinite } \\ \text { Wellbore Radius, } r_{w} & = & 0.1 \mathrm{ft} \\ \quad \text { Formation compressibility, } c_{f} & = & 3 \times 10^{-6} \mathrm{psi}^{-1} \\ \quad \text { Porosity, } \phi & = & 0.05(\text { fraction }) \\ \text { Initial reservoir pressure, } p_{i} & = & 5000 \mathrm{psi} \\ \quad \text { Gas saturation, } s_{g} & = & 1.0 \text { fraction } \\ \quad \text { Skin factor, } s & = & 0.01(\text { dimensionless }) \\ \quad \text { Reservoir temperature, } T_{r} & = & 212^{\circ} \mathrm{F} \\ \text { Fluid properties: } & & \\ \quad \text { Gas specific gravity, } \gamma_{g} & = & 0.7(\text { air }=1) \\ \text { Hydraulically fractured well model } & \text { parameters: } \\ \text { Fracture half-length, } x_{f} & = & 164.0 \mathrm{ft} \\ \quad \text { Number of fractures } & = & 15 \\ \text { Horizontal well length } & = & 6561.7 \mathrm{ft} \\ \text { Production parameters: } & & \\ \text { Flowing pressure, } p_{w f} & = & 500 \mathrm{psia} \\ \text { Production time, } t & = & 10,950 \text { days }(\sim 30 \text { years })\end{array}$

It should be noted that for Modified Wiorkowski Model, a reasonable match of formation permeabilities with time-rate model parameters could not me obtained. Therefore, we present multivariate correlations of Modified Ilk Model only for this case.

\subsubsection{Modified Ilk Model - Parametric Correlations}

In this section we investigate parametric correlating functions between parameters of Modified Ilk Model and 15 numerical simulation cases with varying formation permeabilities. The gas flowrate $q_{g}, D$ - and $b$ parameter plots are given below in Figs. 4.21, 4.22, and 4.23 respectively

Fig. 4.23 also shows a dominant transient/transition flow regime characterized by a negative slope straight line decline of $D$ - parameter on a log-log plot. We observe a slight deviation of trend and change in slope 
at around 100 days. Table 4.9 shows a constant value of zero of parameter $\bar{D}_{\infty}$ for all varying fracture conductivity cases which leads us to its subsequent exclusion from parameter correlation process.

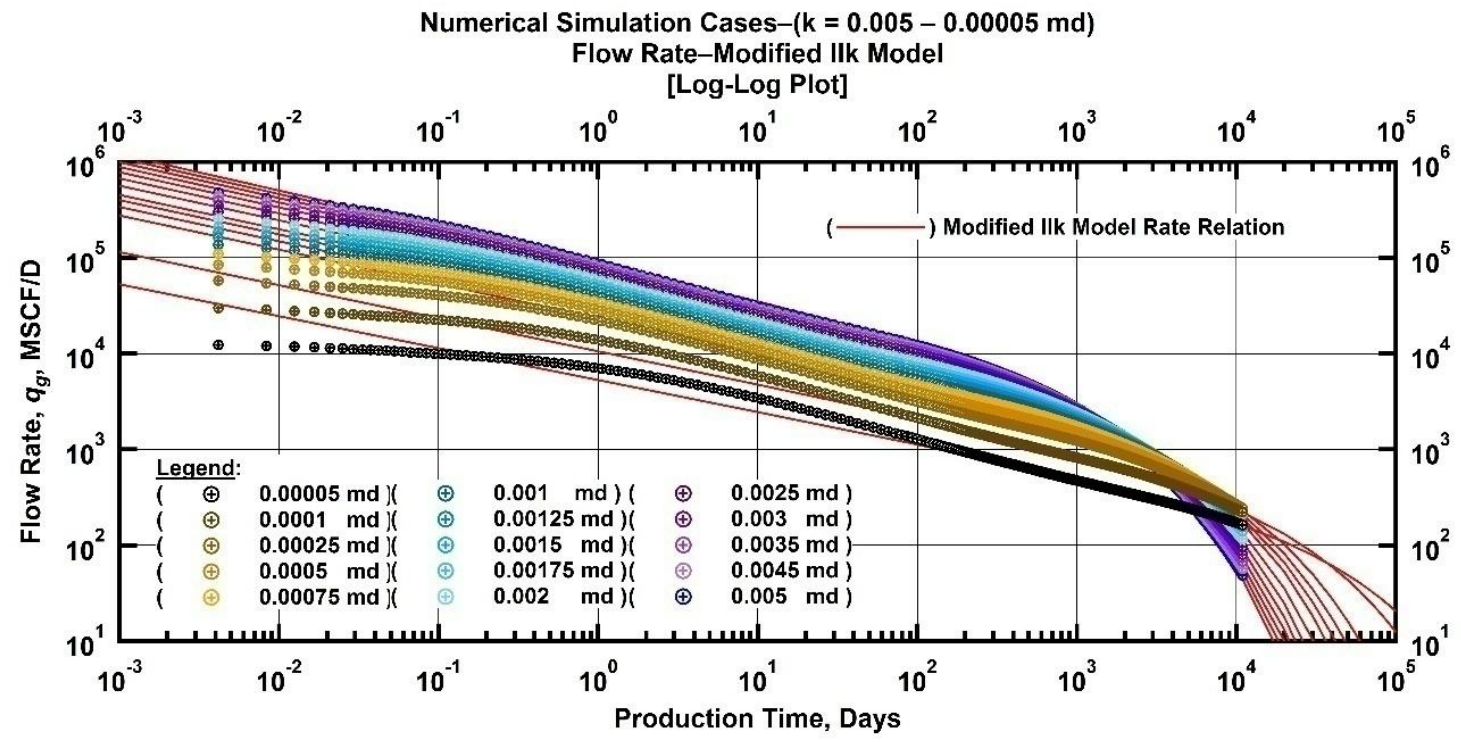

Figure 4.21 - (Log-log Plot): Flow rate $\left(q_{g}\right)$ versus production time. Modified Ilk matches of 15 numerical simulation cases with varying formation permeability.

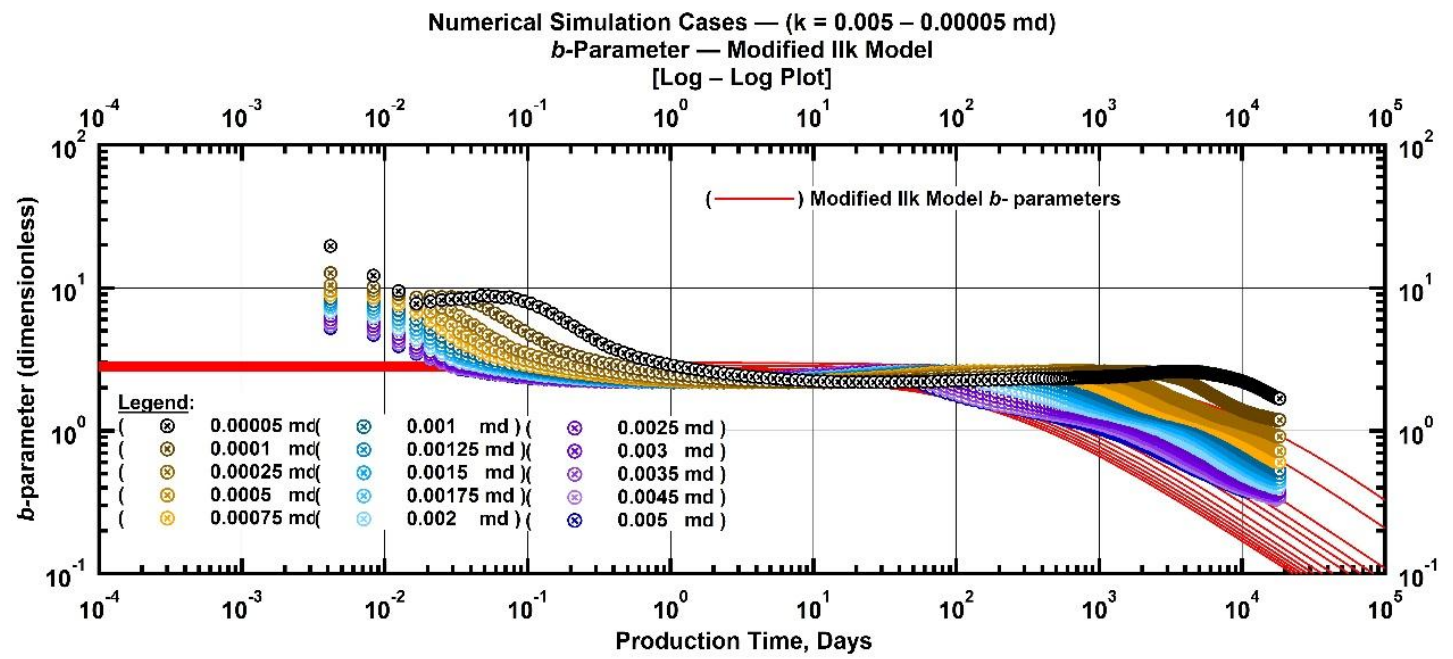

Figure $4.22-\quad$ (Log-log Plot): $b$-parameter versus production time. Modified Ilk model matches of 15 numerical simulation cases with varying formation permeability. 


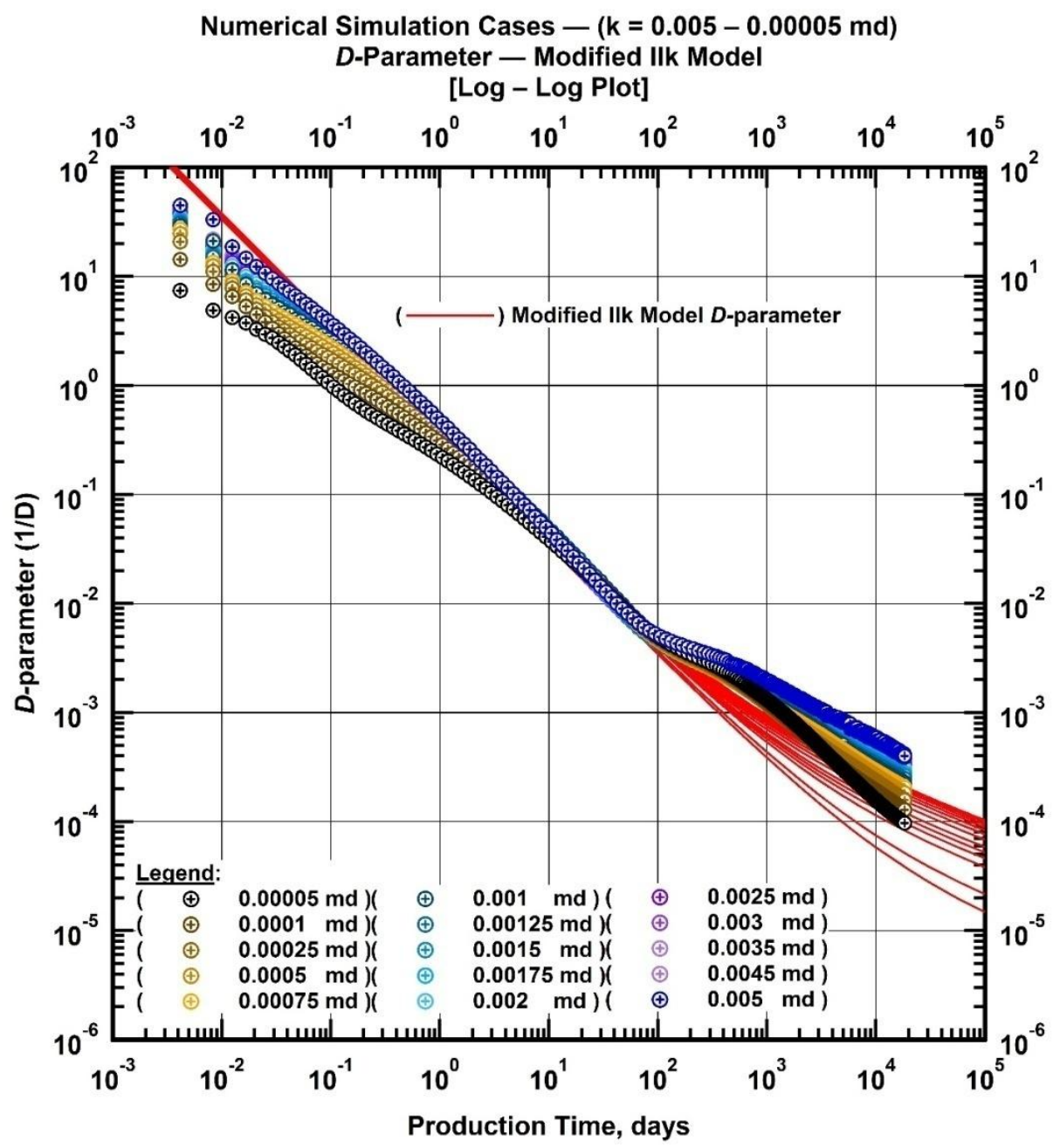

Figure 4.23 - (Log-log Plot): $D$-parameter versus production time. Modified Ilk model matches of 15 numerical simulation cases with varying formation permeability.

The resulting model parameters are given in Table 4.8. 
Table $4.8-$ Modified Ilk Model parameters. Model matches to 15 numerical simulation cases with varying formation permeability.

Num. Sim. Cases

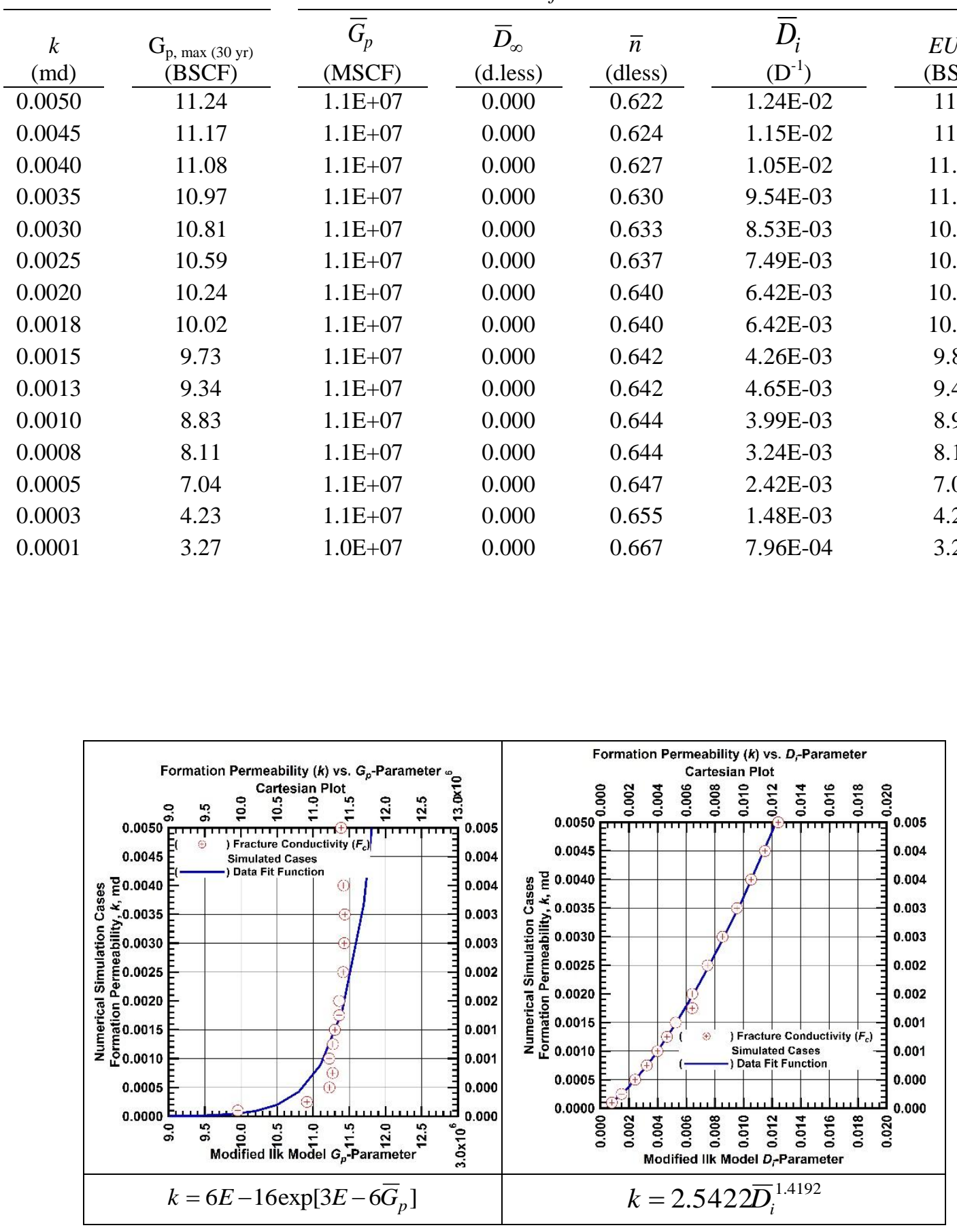

Modified Ilk Model Parameters

$E U R_{M I}$

11.15

11.103

11.035

10.900

10.700

10.400

10.400

9.870

9.455

8.927

8.182

7.040

4.230

3.240 


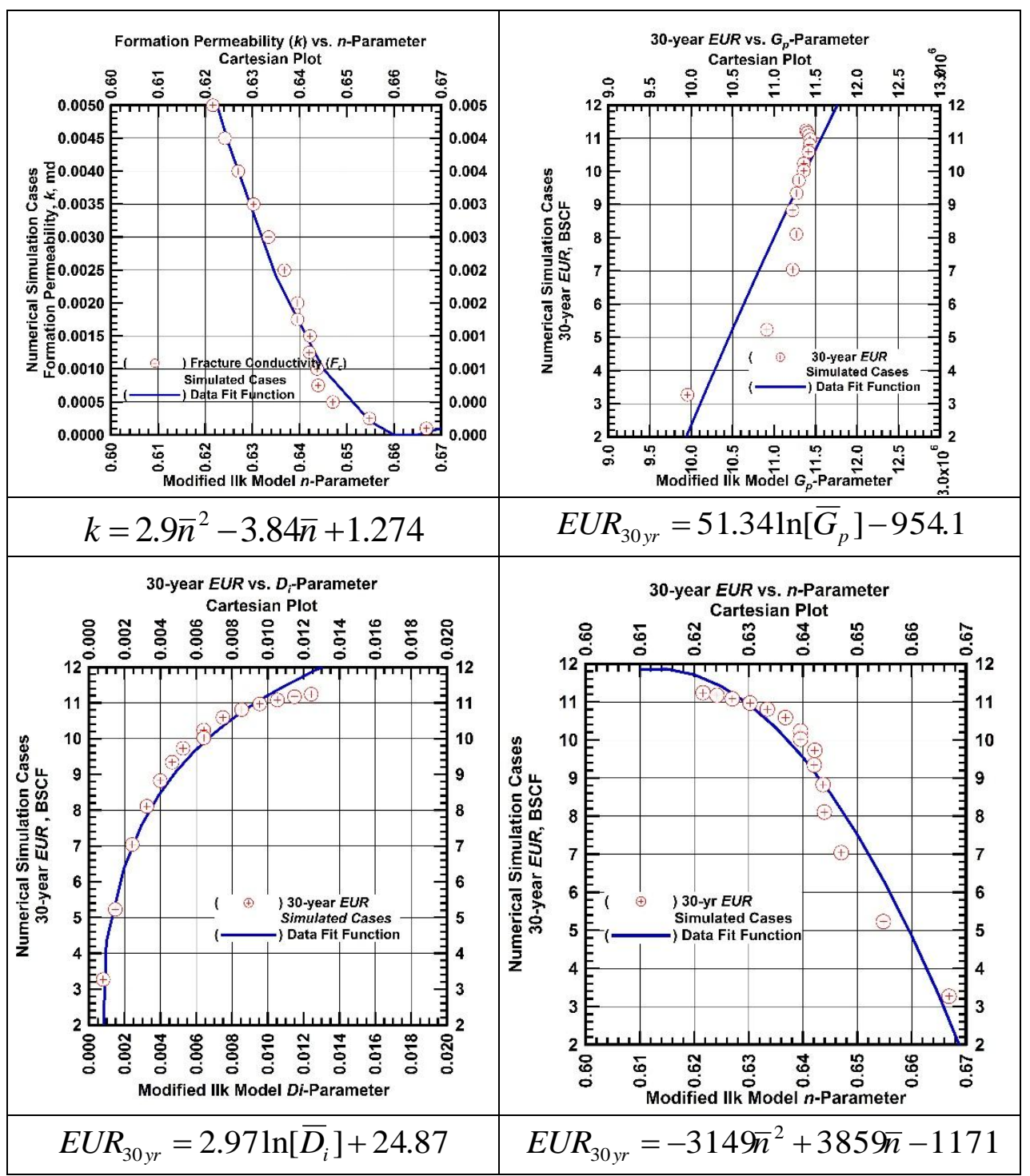

Figure $4.24-$ Cross-plots showing relationship between Modified Ilk model parameters and numerical simulation cases formation permeability $(k)$ and 30 year $E U R$ values.

Fig. 4.24 shows the cross-plots of each considered parameter of Modified Ilk Model parameter for 15 simulation cases against formation permeability and 30-year EUR respectively. These plots show the individual correlating relationship of each model parameter with considered reservoir parameters $(k$ and $\left.E U R_{30 y r}\right)$. We also fit a simple parametric function to cross-plot to determine the underlying correlating function for individual parameters

Fig.4.24 shows that formation permeability can be correlated to model parameters by a combination of linear and logarithmic functions. We propose the correlating function as: 


$$
k=a_{01} \bar{n} \bar{D}_{i}^{a_{02}}
$$

Here, $a_{01}$ and $a_{02}$ are coefficients to be determined through least square regression. Similarly we propose the following correlating function to estimate the 30 year EUR $\left(E U R_{30 y r}\right)$.

$$
E U R_{30 y r}=a_{01} \ln \left[\bar{D}_{i}\right]-a_{02} \bar{n}
$$

Fig. 4.25 shows the resulting model fits for proposed parametric correlations. It can be observed that the proposed correlating functions provide a reliable estimate of the reservoir properties. This indicates that these correlating functions can provide reasonable estimates of the reservoir properties ( $k$ and $\left.E U R_{30} y r\right)$ for the case of a well within the same reservoir system with similar completion and production constraints.

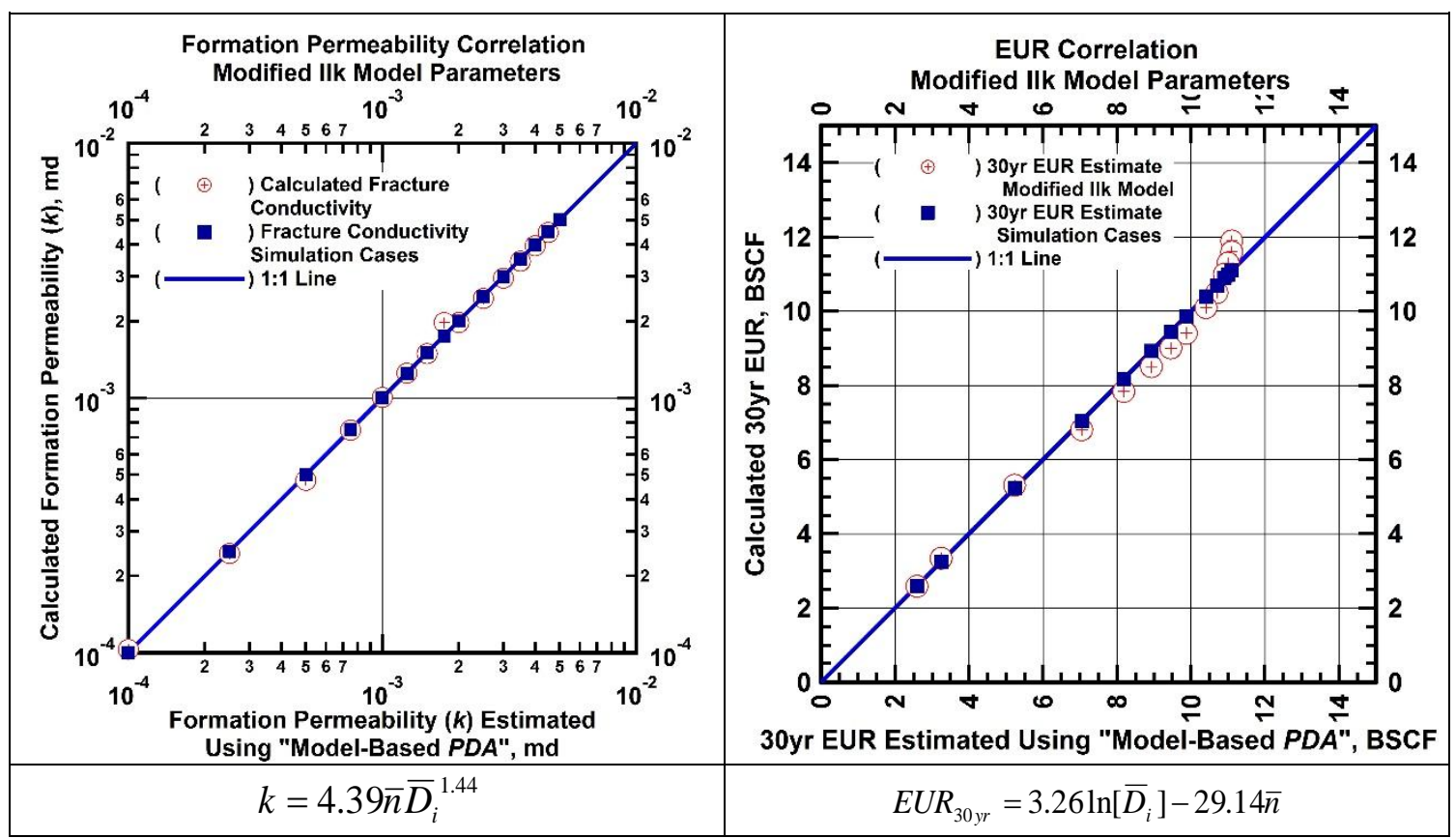

Figure 4.25 - Comparison of fracture conductivity and 30 year EUR values calculated using parametric correlations developed using Modified Ilk model parameters versus numerical simulation models. 


\section{CHAPTER V}

\section{SUMMARY, CONCLUSIONS AND RECOMMENDATIONS FOR FUTURE WORK}

\subsection{Summary}

This work documents the proposal and validation of two new "time-cumulative" relations (along with their respective time-rate relations) for the analysis and interpretation of production performance behavior for unconventional reservoirs (i.e., low/ultra-low permeability reservoirs). These relations are taken from the statistical literature and are based on "self-growth limiting" behavior. The self-growth limiting feature provides an inherent upper limit on recoverable reserves and (should) eliminate the need of a terminal decline as currently required for Arps' time-rate relations.

We also explore the utility of diagnostic functions that are based on growth character of historical cumulative production data (analogous to production rate diagnostic methods). The goal of developing "time-cumulative" diagnostics is to provide characteristic functions which are less influenced by data noise. We provided a methodology to provide specialized diagnostic plots for Modified Wiorkowski Model that allows determination of regression parameters through data character without use of non-linear regression. We tested the methodology for the numerical simulation case.

We have applied the proposed "self-growth limiting" cumulative-time relations and their respective timerate relations for the analysis and interpretation of a number of synthetic and field cases for low/ultra-low permeability (unconventional) reservoir cases. These proposed models provide reasonable matches to historical production data and estimates of EUR. We also presented a performance comparison of proposed new models with widely used rate-time relations found in literature to demonstrate their applicability for matching of transient, transition and boundary-dominated flow regimes. Moreover, we performed Continuous EUR analysis for different wells/completion configurations to determine the rate of convergence to 30-year EUR values (from model based Production analysis) of proposed (MWM and MIM) models.

We also demonstrated the application of a methodology that allows formulating multivariate parametric correlations to integrate completion/reservoir parameters with time-rate model parameters. For this study we considered a number of cases for a low permeability horizontal well with multiple transverse factures. The developed correlations allow estimation of completion/reservoir properties from time-rate model parameters (with the help of benchmark results). We investigated parametric correlations for Modified 
Wiorkowski Model and Modified Ilk Model for fracture conductivity, formation permeability, fracture half-length and 30 year EUR values.

\subsection{Conclusions}

- In this work we present the development of two new "time-rate" relations (i.e., the "ModifiedWiorkowski" and "Modified-Ilk" rate-time and time-cumulative models), both of which are based on self-growth limiting relations. This self-growth limiting feature provides an inherent upper limit on recoverable reserves and eliminates the need for a terminal decline component as required in other time-rate relations (e.g., the modified Arps' models).

- We present the validation and application of the "Modified-Wiorkowski" and "Modified-Ilk" timerate and time-cumulative models. We validate these models against existing time-rate relations (the Arps Exponential decline model, the Arps Hyperbolic decline model, the Power-Law Exponential (PLE) model, the Logistic Growth model (LGM), and the Duong model) using synthetic performance data (i.e., reservoir simulation cases) and field performance data.

- Diagnostics based on cumulative gas production provide smoother diagnostic plots that are comparatively less affected by noise and poor field data.

- In cases where we remove non-linear regression from the model matching process, we can reduce uncertainty in production forecasting and the reserve estimation process.

- Parametric correlations can be formulated to estimate completion/reservoir properties from time-rate model parameters. The established correlations appear to be unique and distinct for corresponding cases.

\subsection{Recommendations for Future Work}

- Efforts should be made to derive diagnostic relations for proposed new models as well as other existing models to determine regression parameters directly from historical production data to eliminate non-linearity and reduce uncertainty.

- Parametric correlation methodology should be expanded to include more well/completion/reservoir parameters. The resulting parametric correlations should be validated on a large number of different field data examples. 


\section{NOMENCLATURE}

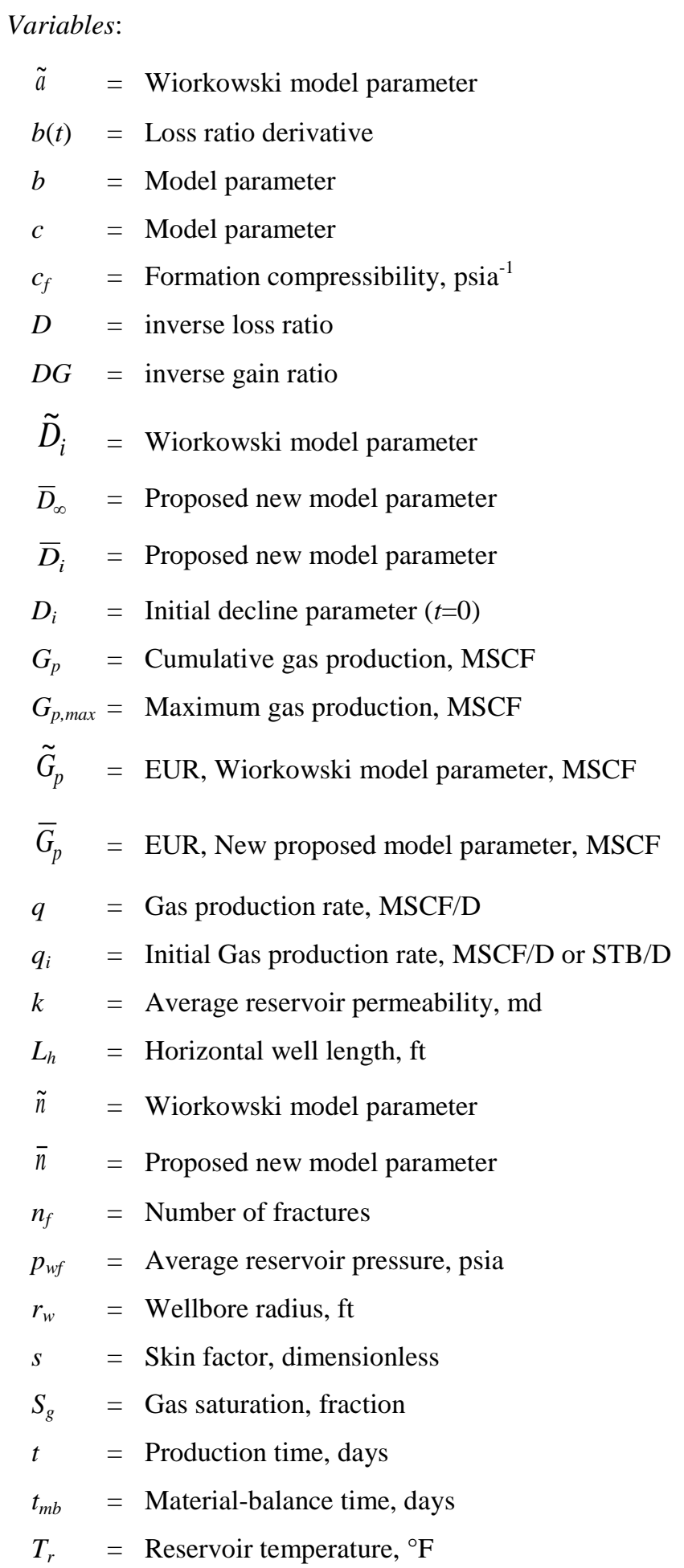


$Y=$ Wiorkowski Model cumulative gas production, MSCF

Dimensionless Variables:

$F_{c D}=$ Dimensionless fracture conductivity

Greek Symbols:

$\gamma_{g}=$ Reservoir gas specific gravity $($ air $=1)$

$\gamma_{W}=$ Model parameter

$\lambda_{W}=$ Model parameter

$\alpha_{W}=$ Model parameter

$\phi \quad=$ Porosity, fraction

Subscripts:

$i=$ Integral function or initial value

$p \quad=$ Produced value

$m b=$ Material balance 


\section{REFERENCES}

Arps J.J. 1945. Analysis of Decline Curves. Trans. AIME: 160, 228-247.

Askabe, Y.A. 2012. Rate-Decline Relation for Unconventional Reservoirs and Development of Parametric Correlations for Estimation of Reservoir Properties. MS Thesis, Department of Petroleum Engineering, Texas A\&M University.

Bourdet, D., Ayoub, J.A., and Pirard, Y.M. 1989. Use of Pressure Derivative in Well-Test Interpretation. SPEFE. 4 (2): 293-302.

Clark, J.A., Lake, L.W. and Patzek, T.W. 2011. Production Forecasting with logistic Growth Models. Paper SPE 144790-MS presented at the SPE Annual Technical Conference and Exhibition, Morgantown, West Virginia, USA 30 October-02 November.

Currie, S. M., Ilk, D., \& Blasingame, T. (2010, January 1). Continuous Estimation of Ultimate Recovery. Society of Petroleum Engineers. doi:10.2118/132352-MS

Doublet, L.E., Pande, P.K, McCollum, T.J., and Blasingame, T.A. 1994. Decline Curve Analysis Using Type Curves-Analysis of Oil Well Production Data Using Material Balance Time: Application to Field Cases. Paper SPE 28688 presented at the International Petroleum Conference and Exhibition of Mexico, Veracruz, Mexico, 10-13 October.

Duong, A.H. 2011. Rate-Decline Analysis for Fracture-Dominated Shale Reservoirs. SPEREE (June): 377-387.

Ecrin Software, Kappa Engineering Software, www.kappaengineering.com

Fetkovich, M.J. 1980. Decline Curve Analysis Using Type Curves. JPT. 32 (6): 1065-1077.

Fulford, D. S., \& Blasingame, T. A. (2013, November 5). Evaluation of Time-Rate Performance of Shale Wells using the Transient Hyperbolic Relation. Society of Petroleum Engineers. doi:10.2118/167242-MS

Hubbert, M. K., Energy Resources, A Report to the Committee on National Resources, National Academy of Sciences-National Research Council, Pub. 1000-D, Washington, DC, 1962.

Ilk, D., Perego, A.D., Rushing, J.A., and Blasingame, T.A. 2008a. Estimating Reserves Using the Arps Hyperbolic Rate-Time Relation - Theory, Practice and Pitfalls. Paper CIM 2008-108 presented at the 
59th Annual Technical Meeting of the Petroleum Society, Calgary, Alberta, Canada, 17-19 June. (in preparation)

Ilk, D., Perego, A.D., Rushing, J.A., and Blasingame, T.A. 2008b. Exponential vs. Hyperbolic Decline in Tight Gas Sands - Understanding the Origin and Implications for Reserve Estimates Using Arps' Decline Curves. Paper SPE 116731 presented at the SPE Annual Technical Conference and Exhibition, Denver, Colorado, 21-24 September.

Ilk, D., Perego, A.D., Rushing, J.A., and Blasingame, T.A. 2008c. Integrating Multiple Production Analysis Techniques To Assess Tight Gas Sand Reserves: Defining a New Paradigm for Industry Best Practices. Paper SPE 114947 presented at the SPE Gas Technology Symposium, Calgary, Alberta, Canada, 17-19 June.

Johnson, R.H. and Bollens, A.L. 1927. The Loss Ratio Method of Extrapolating Oil Well Decline Curves. Trans. AIME 77: 771.

Maley, S. 1985. The Use of Conventional Decline Curve Analysis in Tight Gas Sands. Paper SPE 13898 presented at the SPE Low Permeability Gas Reservoirs Symposium, Denver, CO, May 19-22.

Mishra, S. 2012. A New Approach to Reserves Estimation in Shale Gas Reservoirs Using Multiple Decline Curve Analysis Models. Paper SPE 161092 presented at the SPE Eastern Regional Meeting, Lexington, Kentucky, USA 3-5 October.

Sidle, R. E., \& Lee, W. J. (2011, October 1). Demonstrating Reasonable Certainty Under Principles-Based Oil and Gas Reserves Regulations. Society of Petroleum Engineers. doi:10.2118/146530-PA

Rushing, J.A., Perego, A.D., Sullivan, R.B., and Blasingame, T.A. 2007. Estimating Reserves in Tight Gas Sands at HP/HT Reservoir Conditions: Use and Misuse of an Arps Decline Curve Methodology. Paper SPE 109625 presented at the 2007 Annual SPE Technical Conference and Exhibition, Anaheim, CA., 11 14 November.

Valko, P.P. 2009. Assigning Value to Stimulation in the Barnett Shale: A Simultaneous Analysis of 7000 Plus Production Histories and Well Completion Records. Paper SPE 119369 presented at the SPE Hydraulic Fracturing Technology Conference, Woodlands, TX, January 19-21.

Wiorkowski, J. J., Estimating volumes of remaining fossil fuel resources: a critical review. Journal of the American Statistical Association, 76 (1981), 534-48. 


\section{APPENDIX A}

\section{MODIFIED WIORKOWSKI MODEL — DERIVATIONS}

This model is inspired from the work of Wiorkowski (1981). For matching cumulative oil production data, Wiorkowski proposed a variant of the extended Richards family of growth curves:

$$
Y(t)=C_{W}\left[1+\gamma_{W} \lambda_{W} C_{W} \lambda_{W} \exp \left[\begin{array}{ll}
-\alpha_{W} & t C_{W} \lambda_{W}
\end{array}\right]\right]^{-\frac{1}{\lambda_{W}}}
$$

This is an S-shaped model, and repeated attempts showed that at late-times this model flattens and underestimates the reserves. For unconventional reservoirs, virtually all data are in the transient or transition flow regimes. During boundary-dominated flow we believe that this S-shape feature may act as a self-growth limiting feature.

We propose the following as a practical form of the Wiorkowski model:

$$
G_{p}(t)=\tilde{G}_{p}\left(1-\tilde{a} \exp \left[-\tilde{D}_{i} t\right]\right)^{\tilde{n}}
$$

The modified form of the Wiorkowski model given by Eq. A-1 simplifies the curve fitting process by reducing the number of fitting parameters. The detailed development of the cumulative-time, rate-time, rate-cumulative relations, and the $D$-, $b$ - and $\beta$-derivative functions are given below.

Eq. A-1 can be re-written as follows:

$$
\begin{aligned}
q(t) & =\frac{d G_{p}}{d t}=\frac{d}{d t}\left[\tilde{G}_{p}\left(1-\tilde{a} \exp \left[-\tilde{D}_{i} t\right]\right)^{\tilde{n}}\right] \\
& =\tilde{G}_{p} \frac{d}{d t}\left[\left(1-\tilde{a} \exp \left[-\tilde{D}_{i} t\right]\right)^{\tilde{n}}\right] \\
& =\widetilde{G}_{p} \tilde{n}\left(1-\tilde{a} \exp \left[-\tilde{D}_{i} t\right]\right)^{\tilde{n}-1} \frac{d}{d t}\left[1-\tilde{a} \exp \left[-\tilde{D}_{i} t\right]\right] \\
& =-\tilde{G}_{p} \tilde{n}\left(1-\tilde{a} \exp \left[-\tilde{D}_{i} t\right]\right)^{\tilde{n}-1} \frac{d}{d t}\left[\tilde{a} \exp \left[-\tilde{D}_{i} t\right]\right] \\
& =-\tilde{G}_{p} \tilde{a} \tilde{n}\left(1-\tilde{a} \exp \left[-\tilde{D}_{i} t\right]\right)^{\tilde{n}-1} \frac{d}{d t}\left[\exp \left[-\tilde{D}_{i} t\right]\right] \\
& =\tilde{G}_{p} \tilde{D}_{i} \tilde{a} \tilde{n}\left(1-\tilde{a} \exp \left[-\tilde{D}_{i} t\right]\right)^{\tilde{n}-1} \exp \left[-\tilde{D}_{i} t\right] \\
& =\tilde{G}_{p} \tilde{D}_{i} \tilde{a} \tilde{n} \exp \left[-\tilde{D}_{i} t\right]\left(1-\tilde{a} \exp \left[-\tilde{D}_{i} t\right]\right)^{\tilde{n}-1} \\
& =\tilde{D}_{i} \tilde{a} \tilde{n} \exp \left[-\tilde{D}_{i} t\right]\left(1-\tilde{a} \exp \left[-\tilde{D}_{i} t\right]\right)^{\tilde{n}-1} \tilde{G}_{p}
\end{aligned}
$$

Rearranging Eq. A-1, we can write this expression as:

$$
\frac{G_{p}(t)}{\widetilde{G}_{p}}=\left(1-\tilde{a} \exp \left[-\tilde{D}_{i} t\right]\right)^{\tilde{n}}
$$

Raising both sides of Eq. A-3 to the power of $\frac{1}{\tilde{n}}$, we obtain: 


$$
\left[\frac{G_{p}(t)}{\widetilde{G}_{p}}\right] \frac{1}{\tilde{n}}=1-\tilde{a} \exp \left[-\widetilde{D}_{i} t\right]
$$

Rearranging further, and solving for the exponential term, we have:

$$
\exp \left[-\tilde{D}_{i} t\right]=\frac{1}{\tilde{a}}\left[1-\left[\frac{G_{p}(t)}{\tilde{G}_{p}}\right] \frac{1}{\tilde{n}}\right]
$$

Expanding Eq. A-5 yields:

$$
\frac{G_{p}(t)}{\widetilde{G}_{p}}=\left(1-\tilde{a} \exp \left[-\tilde{D}_{i} t\right]\right)\left(1-\tilde{a} \exp \left[-\widetilde{D}_{i} t\right]\right)^{\tilde{n}-1}
$$

Rearranging this result gives us:

$$
\frac{G_{p}(t)}{1-\tilde{a} \exp \left[-\tilde{D}_{i} t\right]}=\left(1-\tilde{a} \exp \left[-\tilde{D}_{i} t\right]\right)^{\tilde{n}-1} \tilde{G}_{p}
$$

Substituting Eq. A-6 into Eq. A-2, we obtain:

$$
q(t)=\tilde{D}_{i} \tilde{a} \tilde{n} \exp \left[-\tilde{D}_{i} t\right] \frac{G_{p}(t)}{1-\tilde{a} \exp \left[-\tilde{D}_{i} t\right]}
$$

Substituting Eq. A-5 into Eq. A-7 yields:

$$
q(t)=\tilde{D}_{i} \tilde{a} \tilde{n} \frac{1}{\tilde{a}}\left[1-\left[\frac{G_{p}(t)}{\widetilde{G}_{p}}\right] \frac{1}{\tilde{n}}\right]_{1-\tilde{a} \frac{1}{\tilde{a}}\left[1-\left[\frac{G_{p}(t)}{\widetilde{G}_{p}}\right] \frac{1}{\tilde{n}}\right]}^{G_{p}(t)}
$$

Simplifying Eq. A-8, yields the final form the rate-cumulative form for Modified-Wiorkowski Model:

$$
q(t)=\tilde{D}_{i} \tilde{n}\left[\left[\frac{G_{p}(t)}{\tilde{G}_{p}}\right]-\frac{1}{\tilde{n}}-1\right] G_{p}(t)
$$

The definition of the Arps "inverse loss-ratio" is:

$$
D(t)=-\frac{1}{q(t)} \frac{d}{d t}[q(t)] .
$$

Substitution of Eq. A-2 into Eq. A-10 yields:

$$
\begin{aligned}
D(t) & =-\frac{1}{\tilde{G}_{p} \tilde{D}_{i} \tilde{a} \tilde{n} \exp \left[-\tilde{D}_{i} t\right]\left(1-\tilde{a} \exp \left[-\tilde{D}_{i} t\right]\right)^{\tilde{n}-1}} \frac{d}{d t}\left[\tilde{G}_{p} \tilde{D}_{i} \tilde{a} \tilde{n} \exp \left[-\tilde{D}_{i} t\right]\left(1-\tilde{a} \exp \left[-\tilde{D}_{i} t\right]\right)^{\tilde{n}-1}\right] \\
& =-\frac{1}{\exp \left[-\tilde{D}_{i} t\right]\left(1-\tilde{a} \exp \left[-\tilde{D}_{i} t\right]\right)^{\tilde{n}-1}} \frac{d}{d t}\left[\exp \left[-\tilde{D}_{i} t\right]\left(1-\tilde{a} \exp \left[-\tilde{D}_{i} t\right]\right)^{\tilde{n}-1}\right]
\end{aligned}
$$

Recalling the product rule:

$$
\frac{d}{d t}[u v]=u \frac{d v}{d t}+v \frac{d u}{d t}
$$


And continuing the differentiation:

$$
\begin{aligned}
& D(t)=-\frac{1}{\exp \left[-\tilde{D}_{i} t\right]\left(1-\tilde{a} \exp \left[-\tilde{D}_{i} t\right]\right)^{\tilde{n}-1}}\left[\begin{array}{l}
\exp \left[-\tilde{D}_{i} t\right](\tilde{n}-1)\left(1-\tilde{a} \exp \left[-\tilde{D}_{i} t\right]\right)^{\tilde{n}-2} \\
\left(\tilde{a} \tilde{D}_{i} \exp \left[-\tilde{D}_{i} t\right]\right)+\left(1-\tilde{a} \exp \left[-\tilde{D}_{i} t\right]\right)^{\tilde{n}-1}\left(-\tilde{D}_{i} \exp \left[-\tilde{D}_{i} t\right]\right)
\end{array}\right] \\
& =-\frac{\exp \left[-\tilde{D}_{i} t\right]\left(1-\tilde{a} \exp \left[-\tilde{D}_{i} t\right]\right)^{\tilde{n}-1}}{\exp \left[-\tilde{D}_{i} t\right]\left(1-\tilde{a} \exp \left[-\tilde{D}_{i} t\right]\right)^{\tilde{n}-1}}\left[\frac{\tilde{n}-1}{1-\tilde{a} \exp \left[-\tilde{D}_{i} t\right]} \tilde{a} \tilde{D}_{i} \exp \left[-\tilde{D}_{i} t\right]-\tilde{D}_{i}\right] \\
& =-\frac{\tilde{n}-1}{1-\tilde{a} \exp \left[-\widetilde{D}_{i} t\right]} \tilde{a} \tilde{D}_{i} \exp \left[-\tilde{D}_{i} t\right]-\tilde{D}_{i}
\end{aligned}
$$

Simplifying Eq. A-12, we have:

$$
D(t)=\frac{\tilde{D}_{i}\left(-\tilde{a} \tilde{n}+\exp \left[\tilde{D}_{i} t\right]\right)}{-\tilde{a}+\exp \left[\tilde{D}_{i} t\right]}
$$

The definition of the Arps "loss-ratio derivative" is:

$$
b(t)=\frac{d}{d t}\left[\frac{1}{D(t)}\right]
$$

Substituting Eq. A-13 into Eq. A-14, we obtain the following as a starting point:

$$
b(t)=\frac{d}{d t}\left[\frac{-\tilde{a}+\exp \left[\tilde{D}_{i} t\right]}{\tilde{D}_{i}\left(-\tilde{a} \tilde{n}+\exp \left[\tilde{D}_{i} t\right]\right)}\right] .
$$

Recalling the quotient rule;

$$
\frac{d}{d t}\left[\frac{u}{v}\right]=\frac{v \frac{d u}{d t}-u \frac{d v}{d t}}{v^{2}}
$$

Continuing the differentiation:

$$
\begin{aligned}
& b(t)=\frac{\widetilde{D}_{i}\left(-\tilde{a} \tilde{n}+\exp \left[\widetilde{D}_{i} t\right]\right) \frac{d}{d t}\left[-\tilde{a}+\exp \left[\widetilde{D}_{i} t\right]\right]-\left(-\tilde{a}+\exp \left[\widetilde{D}_{i} t\right]\right) \frac{d}{d t}\left[\widetilde{D}_{i}\left(-\tilde{a} \tilde{n}+\exp \left[\widetilde{D}_{i} t\right]\right)\right]}{\left[\widetilde{D}_{i}\left(-\tilde{a} \tilde{n}+\exp \left[\tilde{D}_{i} t\right]\right)\right]^{2}} \\
& =\frac{\tilde{D}_{i}\left(-\tilde{a} \tilde{n}+\exp \left[\tilde{D}_{i} t\right]\right) \tilde{D}_{i} \exp \left[\tilde{D}_{i} t\right]-\left(-\tilde{a}+\exp \left[\tilde{D}_{i} t\right]\right) \tilde{D}_{i} \tilde{D}_{i} \exp \left[\tilde{D}_{i} t\right]}{\left[\tilde{D}_{i}\left(-\tilde{a} \tilde{n}+\exp \left[\tilde{D}_{i} t\right]\right)\right]^{2}} \\
& =\frac{\exp \left[\tilde{D}_{i} t\right]\left(-\tilde{a} \tilde{n}+\exp \left[\tilde{D}_{i} t\right]+\tilde{a}-\exp \left[\tilde{D}_{i} t\right]\right)}{\left[-\tilde{a} \tilde{n}+\exp \left[\tilde{D}_{i} t\right]\right]^{2}}
\end{aligned}
$$

Simplifying Eq. A-17, we have:

$$
b(t)=\frac{\tilde{a}(1-n) \exp \left[\tilde{D}_{i} t\right]}{\left[-\tilde{a} \tilde{n}+\exp \left[\tilde{D}_{i} t\right]\right]^{2}} .
$$

The so-called $\beta$-derivative function is defined as:

$$
\beta(t)=-\frac{t}{q(t)} \frac{d}{d t}[q(t)]
$$


Substituting Eq. A-10 into Eq. A-19 yields an alternative definition:

$$
\beta(t)=t D(t) .
$$

Substituting Eq. A-13 into Eq. A-20 we have the final result for the $\beta$-derivative function:

$$
\beta(t)=\frac{\tilde{D}_{i} t\left(-\tilde{a} \tilde{n}+\exp \left[\tilde{D}_{i} t\right]\right)}{-\tilde{a}+\exp \left[\tilde{D}_{i} t\right]}
$$

\section{References}

1. Wiorkowski, J. J., Estimating volumes of remaining fossil fuel resources: a critical review. Journal of the American Statistical Association, 76 (1981), 534-48. 


\section{APPENDIX B}

\section{MODIFIED ILK MODEL: DERIVATIONS}

The proposed time-cumulative model is a variant of the power-law exponential time-rate relation proposed by Ilk, et al (2008) were the Ilk result was derived by observing that the "inverse loss-ratio" function $(D(t))$ is represented by a power-law function for essentially the entire production period. Using the time-rate form of the Ilk result it was demonstrated that this model matches both transient and transitional flow regimes in low/ultra-low permeability reservoirs. In addition, a constant (terminal) decline parameter $\left(D_{\infty}\right)$ was added to power law relation to match boundary-dominated flow behavior.

Based on the Ilk, et al time-rate model, we propose the following time-cumulative form by induction:

$$
G_{p}(t)=\bar{G}_{p}\left[1-\exp \left[-\bar{D}_{\infty} t-\bar{D}_{i} t^{\bar{n}}\right]\right]
$$

Its derivative can be written as

$$
\begin{aligned}
q(t) & =\frac{d}{d t}\left[G_{p}(t)\right]=\frac{d}{d t}\left[\bar{G}_{p}\left(1-\exp \left[-\bar{D}_{\infty} t-\bar{D}_{i} t^{\bar{n}}\right]\right)\right] \\
& =-\bar{G}_{p} \frac{d}{d t}\left[\exp \left[-\bar{D}_{\infty} t-\bar{D}_{i} t^{\bar{n}}\right]\right] \\
& =-\bar{G}_{p} \exp \left[-\bar{D}_{\infty} t-\bar{D}_{i} t^{\bar{n}}\right]\left[-\bar{D}_{\infty}-\bar{n} \bar{D}_{i} t^{\bar{n}-1}\right]
\end{aligned}
$$

Where the most compact form of this expression is given as:

$$
q(t)=\bar{G}_{p} \exp \left[-\bar{D}_{\infty} t-\bar{D}_{i} t^{\bar{n}}\right]\left[\bar{D}_{\infty}+\bar{n} \bar{D}_{i} t^{\bar{n}-1}\right]
$$

Where Eq. B-3 is the preferred time-rate form of the Modified-Ilk Model. Dividing Eq. B-3 (time-rate model) by Eq. B-1 (time-cumulative model), we have:

$$
\begin{aligned}
\frac{q(t)}{G_{p}(t)} & =\frac{\bar{G}_{p} \exp \left[-\bar{D}_{\infty} t-\bar{D}_{i} t^{\bar{n}}\right]\left[\bar{D}_{\infty}+\bar{n} \bar{D}_{i} t^{\bar{n}-1}\right]}{\bar{G}_{p}\left[1-\exp \left[-\bar{D}_{\infty} t-\bar{D}_{i} t^{\bar{n}}\right]\right]} \\
& =\frac{\exp \left[-\bar{D}_{\infty} t-\bar{D}_{i} t^{\bar{n}}\right]\left[\bar{D}_{\infty}+\bar{n} \bar{D}_{i} t^{\bar{n}-1}\right]}{\left[1-\exp \left[-\bar{D}_{\infty} t-\bar{D}_{i} t^{\bar{n}}\right]\right]}
\end{aligned}
$$

Simplifying Eq. B-4 to yield the most compact form, we obtain:

$$
\frac{q(t)}{G_{p}(t)}=\frac{\bar{D}_{\infty} t+\bar{n} \bar{D}_{i} t^{\bar{n}}}{t\left[\exp \left[\bar{D}_{\infty} t+\bar{D}_{i} t^{\bar{n}}\right]-1\right]}
$$

The definition of the Arps "inverse loss-ratio" is:

$$
D(t)=-\frac{1}{q(t)} \frac{d}{d t}[q(t)]
$$




$$
\begin{aligned}
D(t) & =-\frac{1}{-\bar{G}_{p} \exp \left[-\bar{D}_{\infty} t-\bar{D}_{i} t^{\bar{n}}\right]\left[-\bar{D}_{\infty}-\bar{n} \bar{D}_{i} t^{\bar{n}-1}\right]} \frac{d}{d t}\left[-\bar{G}_{p} \exp \left[-\bar{D}_{\infty} t-\bar{D}_{i} t^{\bar{n}}\right]\left[-\bar{D}_{\infty}-\bar{n} \bar{D}_{i} t^{\bar{n}-1}\right]\right] \\
& =-\frac{1}{\exp \left[-\bar{D}_{\infty} t-\bar{D}_{i} \bar{t}^{\bar{n}}\right]\left[-\bar{D}_{\infty}-\bar{n} \bar{D}_{i} t^{\bar{n}-1}\right]} \frac{d}{d t}\left[\exp \left[-\bar{D}_{\infty} t-\bar{D}_{i} t^{\bar{n}}\right]\left[-\bar{D}_{\infty}-\bar{n} \bar{D}_{i} t^{\bar{n}-1}\right]\right]
\end{aligned}
$$

Recalling the product rule:

$$
\frac{d}{d t}[u v]=u \frac{d v}{d t}+v \frac{d u}{d t}
$$

Continuing the differentiation:

$$
\begin{aligned}
& D(t)=-\frac{1}{\exp \left[-\bar{D}_{\infty} t-\bar{D}_{i} t^{\bar{n}}\right]\left[-\bar{D}_{\infty}-\bar{n} \bar{D}_{i} t^{\bar{n}-1}\right]}\left[\begin{array}{l}
\exp \left[-\bar{D}_{\infty} t-\bar{D}_{i} t^{\bar{n}}\right] \frac{d}{d t}\left[-\bar{D}_{\infty}-\bar{n} \bar{D}_{i} t^{\bar{n}-1}\right] \\
+\left[-\bar{D}_{\infty}-\bar{n} \bar{D}_{i} t^{\bar{n}-1}\right] \frac{d}{d t}\left[\exp \left[-\bar{D}_{\infty} t-\bar{D}_{i} t^{\bar{n}}\right]\right]
\end{array}\right] \\
& =-\frac{1}{\exp \left[-\bar{D}_{\infty} t-\bar{D}_{i} t^{\bar{n}}\right]\left[-\bar{D}_{\infty}-\bar{n} \bar{D}_{i} t^{\bar{n}-1}\right]}\left[\begin{array}{l}
\exp \left[-\bar{D}_{\infty} t-\bar{D}_{i} t^{\bar{n}}\right]\left[-\bar{n}(\bar{n}-1) \bar{D}_{i} t^{\bar{n}-2}\right] \\
+\left[-\bar{D}_{\infty}-\bar{n} \bar{D}_{i} t^{\bar{n}-1}\right]^{2}\left[\exp \left[-\bar{D}_{\infty} t-\bar{D}_{i} t^{\bar{n}}\right]\right]
\end{array}\right] \\
& =-\frac{1}{\left[-\bar{D}_{\infty}-\bar{n} \bar{D}_{i} t^{\bar{n}-1}\right]}\left[\left[-\bar{n}(\bar{n}-1) \bar{D}_{i} t^{\bar{n}-2}\right]+\left[-\bar{D}_{\infty}-\bar{n} \bar{D}_{i} t^{\bar{n}-1}\right]^{2}\right] \\
& =\frac{1}{\left[\bar{D}_{\infty}+\bar{n} \bar{D}_{i} t^{\bar{n}-1}\right]}\left[\bar{n} \bar{D}_{i} t^{\bar{n}-2}-\bar{n}^{2} \bar{D}_{i} t^{\bar{n}-2}+\bar{D}_{\infty}{ }^{2}+2 \bar{n} \bar{D}_{\infty} \bar{D}_{i} t^{\bar{n}-1}+\left(\bar{n} \bar{D}_{i} t^{\bar{n}-1}\right)^{2}\right] \\
& =\frac{1}{t\left[\bar{D}_{\infty} t+\bar{n} \bar{D}_{i} t^{\bar{n}}\right]}\left[\bar{D}_{\infty}{ }^{2} t^{2}+2 \bar{D}_{\infty} \bar{D}_{i} \bar{n} t^{1-\bar{n}}+\bar{D}_{i} \bar{n} t^{\bar{n}}\left[1+\bar{n}\left(\bar{D}_{i} t^{\bar{n}}-1\right)\right]\right] \\
& =\frac{\bar{n} \bar{D}_{i} t^{\bar{n}-2}-\bar{n}^{2} \bar{D}_{i} t^{\bar{n}-2}}{\left[\bar{D}_{\infty}+\bar{n} \bar{D}_{i} t^{\bar{n}-1}\right]}+\frac{\bar{D}_{\infty}{ }^{2}+2 \bar{n} \bar{D}_{\infty} \bar{D}_{i} t^{\bar{n}-1}}{\left[\bar{D}_{\infty}+\bar{n} \bar{D}_{i} t^{\bar{n}-1}\right]}+\frac{\left(\bar{n} \bar{D}_{i} t^{\bar{n}-1}\right)^{2}}{\left[\bar{D}_{\infty}+\bar{n} \bar{D}_{i} t^{\bar{n}-1}\right]}
\end{aligned}
$$

Reducing to the most compact algebraic form, we have:

$$
D(t)=\bar{D}_{\infty}+\frac{1-\bar{n}+\bar{D}_{i} \bar{n} t}{t}+\frac{(\bar{n}-1) \bar{D}_{\infty}}{\left[\bar{D}_{\infty} t+\bar{n} \bar{D}_{i} t^{\bar{n}}\right]}
$$

The definition of the Arps "loss-ratio derivative" is:

$$
b(t)=\frac{d}{d t}\left[\frac{1}{D(t)}\right]
$$

The definition of the Arps "loss-ratio derivative" is:

$$
b(t)=\frac{d}{d t}\left[\frac{t\left[\bar{D}_{\infty} t+\bar{n} \bar{D}_{i} t^{\bar{n}}\right]}{\bar{D}_{\infty}{ }^{2} t^{2}+2 \bar{D}_{\infty} \overline{D_{i}} \bar{n} t^{1-\bar{n}}+\bar{D}_{i} \bar{n} t^{\bar{n}}\left[1+\bar{n}\left(\bar{D}_{i} t^{\bar{n}}-1\right)\right]}\right]
$$

Recalling the quotient rule; 


$$
\frac{d}{d t}\left[\frac{u}{v}\right]=\frac{v \frac{d u}{d t}-u \frac{d v}{d t}}{v^{2}}
$$

Continuing the differentiation:

$$
\begin{aligned}
& {\left[\bar{D}_{\infty}{ }^{2} t^{2}+2 \bar{D}_{\infty} \overline{D_{i}} \bar{n} t^{1-\bar{n}}+\bar{D}_{i} \bar{n} t^{\bar{n}}\left[1+\bar{n}\left(\bar{D}_{i} t^{\bar{n}}-1\right)\right]\right] \frac{d}{d t}\left[t\left[\bar{D}_{\infty} t+\bar{n} \bar{D}_{i} t^{\bar{n}}\right]\right]} \\
& b(t)=\frac{-t\left[\bar{D}_{\infty} t+\bar{n} \bar{D}_{i} t^{\bar{n}}\right] \frac{d}{d t}\left[\bar{D}_{\infty}{ }^{2} t^{2}+2 \bar{D}_{\infty} \bar{D}_{i} \bar{n} t^{1-\bar{n}}+\bar{D}_{i} \bar{n} t^{\bar{n}}\left[1+\bar{n}\left(\bar{D}_{i} t^{\bar{n}}-1\right)\right]\right]}{\left[\bar{D}_{\infty}{ }^{2} t^{2}+2 \bar{D}_{\infty} \bar{D}_{i} \bar{n} t^{1-\bar{n}}+\bar{D}_{i} \bar{n} t^{\bar{n}}\left[1+\bar{n}\left(\bar{D}_{i} t^{\bar{n}}-1\right)\right]\right]^{2}}
\end{aligned}
$$

Simplifying Eq. B-14, we have:

$$
b(t)=-\frac{\bar{D}_{i}(\bar{n}-1) \bar{n} t^{\bar{n}}\left[\bar{D}_{i}{ }^{2} \bar{n}^{2} t^{2 \bar{n}}+\bar{D}_{\infty} t\left(2-\bar{n}+\bar{D}_{\infty} t\right)+\bar{D}_{i} \bar{n} t^{\bar{n}}\left(1+2 \bar{D}_{\infty} t\right)\right]}{\left[\bar{D}_{\infty}{ }^{2} t^{2}+2 \bar{D}_{\infty} \bar{D}_{i} \bar{n} t^{1-\bar{n}}+\bar{D}_{i} \bar{n} t^{\bar{n}}\left[1+\bar{n}\left(\bar{D}_{i} t^{\bar{n}}-1\right)\right]\right]^{2}}
$$

The so-called $\beta$-derivative function is defined as:

$$
\beta(t)=-\frac{t}{q(t)} \frac{d}{d t}[q(t)]
$$

Substituting Eq. B-6 into Eq. B-16 yields an alternative definition:

$$
\beta(t)=t D(t)
$$

Substituting Eq. B-10 into Eq. B-17 we have the final result for the $\beta$-derivative function:

$$
\beta(t)=t\left[\bar{D}_{\infty}+\frac{1-\bar{n}+\bar{D}_{i} \bar{n} t}{t}+\frac{(\bar{n}-1) \bar{D}_{\infty}}{\left[\bar{D}_{\infty} t+\bar{n} \bar{D}_{i} t^{\bar{n}}\right]}\right]
$$

Multiplying through by $t$ on a term-by-term basis yields our final result:

$$
\beta(t)=\bar{D}_{\infty} t+1-\bar{n}+\bar{D}_{i} \bar{n} t^{\bar{n}}+\frac{(\bar{n}-1) \bar{D}_{\infty} t}{\left(\bar{D}_{\infty} t+\bar{n} \bar{D}_{i} t^{\bar{n}}\right)}
$$

\section{References}

1.Ilk, D., Rushing, J.A., and Blasingame, T.A. 2008. Estimating Reserves Using the Arps Hyperbolic Rate-Time Relation - Theory, Practice and Pitfalls. Paper CIM 2008-108 presented at the 59th Annual Technical Meeting of the Petroleum Society, Calgary, Alberta, Canada, 17-19 June. (in preparation) 


\section{APPENDIX C}

\section{DEVELOPMENT OF TIME-CUMULATIVE PRODUCTION DIAGNOSTICS}

Analogous to diagnostics $D$-, $b$ - and $\beta$-derivative, which are essentially decline functions, we propose diagnostics derived for cumulative-time relations as cumulative production diagnostics. As the nature of cumulative function is 'incremental' over time, these diagnostics can be stated in terms of "gain-ratio", instead of "loss-ratio".

These functions are proposed as,

$$
D_{c}(t)=\frac{1}{G_{p}(t)} \frac{d G_{p}(t)}{d t} \ldots \ldots \ldots \ldots \ldots \ldots \ldots \ldots \ldots . . .(\text { reciprocal of the cumulative loss-ratio }) .
$$

And,

$$
b_{c}(t)=\frac{d}{d t}\left[\frac{1}{D_{c}(t)}\right]=\frac{d}{d t}\left[\frac{G_{p}(t)}{d D_{c}(t) / d t}\right] \text { (derivative of the cumulative loss-ratio). }
$$

Eq. C-1 can be rewritten as,

$$
D_{c}(t)=\frac{1}{G_{p}(t)} q(t)
$$

Or in terms of the material balance time, $t_{m b}$, we have:

$$
D_{c}(t)=\frac{1}{t_{m b}}
$$

Similarly, Eq. C-2 can be rewritten as,

$$
b_{c}(t)=\frac{d}{d t}\left[\frac{1}{D_{c}(t)}\right]=\frac{d}{d t}\left[t_{m b}\right]
$$

Note that both $D_{c}(t)$ and $b_{c}(t)$ can be described solely as a function of material-balance time $\left(t_{m b}\right) . D_{c}(t)$ or rather $D_{c}\left(t_{m b}\right)$ exhibit same straight-line behavior for transient data as $D$-parameter for our numerical simulation Base Case. This parameter is basically driven by cumulative relation which is not as sensitive to variations or noise as rate relations. Therefore, for the test case we plotted, it shows comparatively less deviation from the normal transient trend than that of $D$ - parameter. This shows that new cumulative production diagnostics provide comparatively smoother diagnostic functions which are less affected by noise in production data. Thus, the underlying character of the data is stronger for cumulative production diagnostics. 


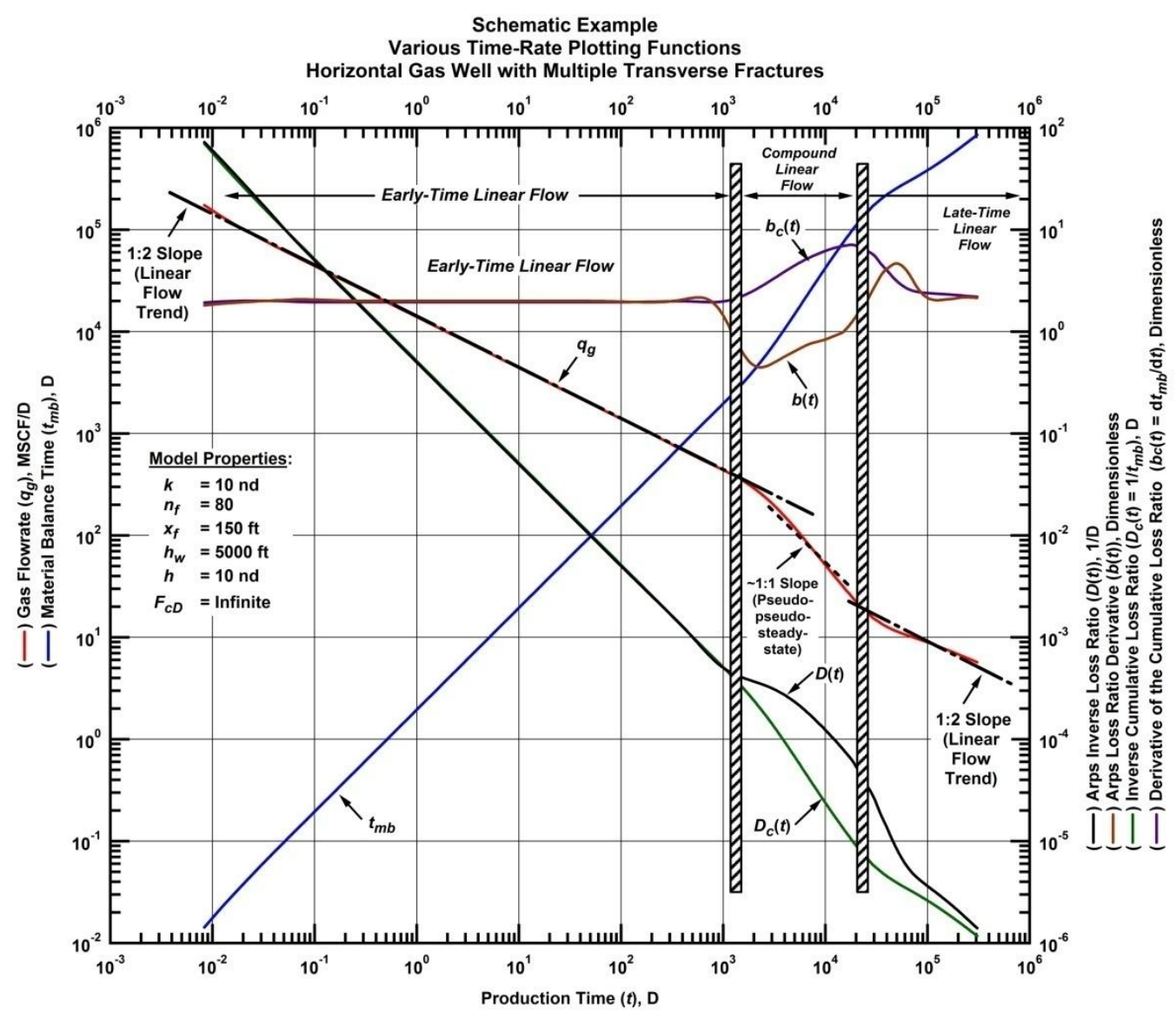

Figure C-1 - (Log-log Plot): Schematic example of time-rate plotting functions for a horizontal gas well with multiple transverse fractures (numerical simulation case).

\section{C.1 Development of Traditional Decline Diagnostics In Terms of Cumulative Production Diagnostics}

In this section, we attempt to investigate that whether we can express "loss-ratio" diagnostic functions in terms of "cumulative loss-ratio" diagnostic functions. Also, it should be noted that since all 'cumulative loss-ratio' diagnostics can be expressed as functions of material-balance time $\left(t_{m b}\right)$, such a relation would result in expressing loss-ratio diagnostics as a function of material-balance time $\left(t_{m b}\right)$ as well.

From Eq. C-3, we can write,

$$
q(t)=G_{p}(t) D_{c}(t)
$$

'inverse loss-ratio' is defined as,

$$
D(t)=-\frac{1}{q(t)} \frac{d}{d t}[q(t)]
$$

Substituting flow rate from Eq. C-7 we get, 


$$
D(t)=-\frac{1}{G_{p}(t) D_{c}(t)} \frac{d}{d t}\left[G_{p}(t) D_{c}(t)\right]
$$

Using product rule;

$$
\frac{d}{d t}[u v]=u \frac{d v}{d t}+v \frac{d u}{d t}
$$

We get,

$$
D(t)=-\frac{1}{G_{p}(t) D_{c}(t)}\left[D_{c}(t) \frac{d}{d t}\left[G_{p}(t)\right]+G_{p}(t) \frac{d}{d t}\left[D_{c}(t)\right]\right] .
$$

Using Eq. C-4, we get

$$
\begin{aligned}
& D(t)=-\frac{t_{m b}}{G_{p}(t)}\left[\frac{1}{t_{m b}} q(t)+G_{p}(t) \frac{d}{d t}\left[\frac{1}{t_{m b}}\right]\right] \\
& D(t)=-\left[\frac{q(t)}{G_{p}(t)}+t_{m b} \frac{d}{d t}\left[\frac{1}{t_{m b}}\right]\right] .
\end{aligned}
$$

Using definition of material balance time as,

$$
t_{m b}=\frac{G_{p}(t)}{q(t)}
$$

Thus, we get,

$$
D(t)=-\left[\frac{1}{t_{m b}}+t_{m b} \frac{d}{d t}\left[\frac{1}{t_{m b}}\right]\right] .
$$

Note that above equation describes $D(t)$ as function of material-balance time only.

Similarly, 'loss-ratio derivative' can be defined as,

$$
b(t)=\frac{d}{d t}\left[\frac{1}{D(t)}\right]
$$

From Eq. C-14, we can write

$$
\frac{1}{D(t)}=-\left[\frac{1+t_{m b}^{2} d\left[1 / t_{m b}\right] / d t}{t_{m b}}\right] .
$$

Thus, Eq. C-15 becomes,

$$
b(t)=\frac{d}{d t}\left[-\frac{1+t_{m b}^{2} d\left[1 / t_{m b}\right] / d t}{t_{m b}}\right]
$$

By quotient rule, 


$$
\frac{d}{d t}\left[\frac{u}{v}\right]=\frac{v \frac{d u}{d t}-u \frac{d v}{d t}}{v^{2}}
$$

Eq. C-17 becomes,

$$
b(t)=\frac{t_{m b} \frac{d}{d t}\left[1+t_{m b}^{2} d\left[1 / t_{m b}\right] / d t\right]-\left(1+t_{m b}^{2} d\left[1 / t_{m b}\right] / d t\right) \frac{d t_{m b}}{d t}}{t_{m b}{ }^{2}}
$$

Again, using product rule as in Eq. C-9, we get

$$
\begin{aligned}
& b(t)=\frac{t_{m b}\left[2 t_{m b} d\left[1 / t_{m b}\right] / d t+t_{m b}{ }^{2} d^{2}\left[1 / t_{m b}\right] / d t^{2}\right]-\left(\frac{d t_{m b}}{d t}+t_{m b}{ }^{2} \frac{d t_{m b}}{d t} d\left[1 / t_{m b}\right] / d t\right)}{t_{m b}{ }^{2}} \\
& b(t)=\frac{2 t_{m b}{ }^{2} d\left[1 / t_{m b}\right] / d t+t_{m b}{ }^{3} d^{2}\left[1 / t_{m b}\right] / d t^{2}-\frac{d t_{m b}}{d t}-t_{m b}{ }^{2} \frac{d t_{m b}}{d t} d\left[1 / t_{m b}\right] / d t}{t_{m b}{ }^{2}} \\
& b(t)=2 \frac{d}{d t}\left[\frac{1}{t_{m b}}\right]+t_{m b} \frac{d^{2}}{d t^{2}}\left[\frac{1}{t_{m b}}\right]-\frac{1}{t_{m b}^{2}} \frac{d t_{m b}}{d t}-\frac{d t_{m b}}{d t} \frac{d}{d t}\left[\frac{1}{t_{m b}}\right] \\
& b(t)=\frac{d}{d t}\left[\frac{1}{t_{m b}}\right]\left[2-\frac{d t_{m b}}{d t}\right]+t_{m b} \frac{d^{2}}{d t^{2}}\left[\frac{1}{t_{m b}}\right]-\frac{1}{t_{m b}^{2}} \frac{d t_{m b}}{d t}
\end{aligned}
$$

Eq. C-23 is the derived form of 'loss-ratio derivative' as a function of material-balance time only.

Alternatively, Eq. C-14 and Eq. C-23can be written in terms of 'cumulative loss-ratio' and 'cumulative loss-ratio derivative' as,

$$
\begin{aligned}
& D(t)=-\left[D_{c}(t)+\frac{1}{D_{c}(t)} \frac{d}{d t}\left[D_{c}(t)\right]\right] \\
& b(t)=\frac{d}{d t}\left[D_{c}(t)\right]\left[2-b_{c}(t)\right]+\frac{1}{D_{c}(t)} \frac{d^{2}}{d t^{2}}\left[D_{c}(t)\right]-\left[D_{c}(t)\right]^{2} b_{c}(t)
\end{aligned}
$$




\section{APPENDIX D}

\section{A METHODOLOGY TO REDUCE UNCERTAINTY IN EUR ESTIMATION FOR MODIFIED WIORKOWSKI MODEL}

In this section, we will derive 'gain-ratio' diagnostic functions for Modified Wiorkowski Model. Moreover, we will express a methodology by which model parameters $b$ and $c$ can be determined from historical production data directly without regression. This will enable us to determine EURs by analyzing character of historical data and thereby, reducing uncertainty and non-uniqueness in EUR estimation.

Recall cumulative-time relations for Modified Wiorkowski Model as,

$$
G_{p}(t)=\tilde{G}_{p}\left(1-\tilde{a} \exp \left[-\tilde{D}_{i} t\right]\right)^{\tilde{n}}
$$

Where $\tilde{G}_{p}, \tilde{a}, \widetilde{D}_{i}$ and $\tilde{n}$ are fit parameters obtained by least squares regression.

Definitions of 'inverse gain-ratio', $D_{c}(t)$, and 'gain-ratio derivative', $b_{c}(t)$, for Modified Wiorkowski Model can be given as

$$
\begin{aligned}
& D_{c}(t)=\frac{\tilde{a} \tilde{D}_{i} \tilde{n}}{\exp \left[-\tilde{D}_{i} t\right]-\tilde{a}} \\
& b_{c}(t)=\frac{\exp \left[-\widetilde{D}_{i} t\right]}{\tilde{a} \tilde{n}} \ldots \ldots . .
\end{aligned}
$$

It was observed that with a little mathematical manipulation, the decline and growth diagnostic parameters can be used to independently determine parameters of Modified Wiorkowski Model. This will effectively make all parameters of this model an original character of historical data. The result is an estimation of EUR independent of non-linear regression for each data set. It should be noted that earlier it was observed that parameter $a$ has only fine tuning effects on model match, and thus it can be set equal to unity for most cases. For parameter $\widetilde{D}_{i}$ and $\tilde{n}$, we propose this relation,

$$
D_{c}(t) b_{c}(t)=\frac{d t_{m b} / d t}{t_{m b}}=\frac{1}{\tilde{n}} \frac{1}{t_{m b}}+\tilde{D}_{i}
$$

To derive the relation in Eq. D-4, let's start with Eq. D-3. With little mathematical manipulation, it can be rewritten as

$$
b_{c}(t)=-\tilde{D}_{i} \frac{\tilde{a}-\exp \left[-\tilde{D}_{i} t\right]-\tilde{a}}{\tilde{a} \tilde{n} \tilde{D}_{i}}
$$

Formulating to separate $D_{c}(t)$ out of Eq. D-5, we can write, 


$$
b_{c}(t)=-\tilde{D}_{i}\left[\frac{\tilde{a}-\exp \left[-\tilde{D}_{i} t\right]}{\tilde{a} \tilde{n} \tilde{D}_{i}}-\frac{\tilde{a}}{\tilde{a} \tilde{n} \tilde{D}_{i}}\right]
$$

By using Eq. D-2, Eq. D-6 becomes

$$
b_{c}(t)=\tilde{D}_{i}\left[\frac{1}{D_{c}(t)}+\frac{1}{\tilde{n} \widetilde{D}_{i}}\right]
$$

Or,

$$
b_{c}(t)=\frac{\widetilde{D}_{i}}{D_{c}(t)}+\frac{1}{\tilde{n}}
$$

With a little mathematical manipulation, we can rewrite Eq. D-8 as,

$$
D_{c}(t) b_{c}(t)=\tilde{D}_{i}+\frac{D_{c}(t)}{\tilde{n}}
$$

Using definitions of $D_{c}(t)$ and $b_{c}(t)$ as given in Eq. D-2 and Eq. D-3, we get

$$
\frac{d t_{m b} / d t}{t_{m b}}=\frac{1}{\tilde{n}} \frac{1}{t_{m b}}+\widetilde{D}_{i}
$$

Thus, a plot of $\frac{d t_{m b} / d t}{t_{m b}}$ vs $\frac{1}{t_{m b}}$ or $D_{c}(t)$ gives $1 / \tilde{n}$ as slope and $\widetilde{D}_{i}$ as intercept. With these two parameters determined, and $a$ equal to unity, we can visually match historical rate-time and cumulativetime production data with rate-time and cumulative-time relations of Modified Wiorkowski Model as given in Eq. D-1, by adjusting EUR values. It is recommended to get best match by visual inspection of both rate-time and cumulative time matches.

From Eq. D-10, parameter $\tilde{n}$ can be defined from the definition of slope as,

$$
\frac{1}{\tilde{n}}=\frac{\Delta\left[\left(d t_{m b} / d t\right) / t_{m b}\right]}{\Delta t_{m b}}
$$

Or,

$$
\tilde{n}=\frac{\Delta t_{m b}}{\Delta\left[\left(d t_{m b} / d t\right) / t_{m b}\right]}
$$

Using Eq. D-10 and Eq. D-12, parameter $\widetilde{D}_{i}$ can be written as,

$$
\tilde{D}_{i}=\frac{d t_{m b} / d t}{t_{m b}}-\frac{\Delta\left[\left(d t_{m b} / d t\right) / t_{m b}\right]}{\Delta t_{m b}} \frac{1}{t_{m b}}
$$

Or, 


$$
\widetilde{D}_{i}=\frac{1}{t_{m b}}\left[\frac{d t_{m b}}{d t}-\frac{\Delta\left[\left(d t_{m b} / d t\right) / t_{m b}\right]}{\Delta t_{m b}}\right]
$$

Fig. D-1 gives a plot of material-balance time and inverse material-balance time versus time. Note that inverse material-balance time is effectively, $D_{c}(t)$.

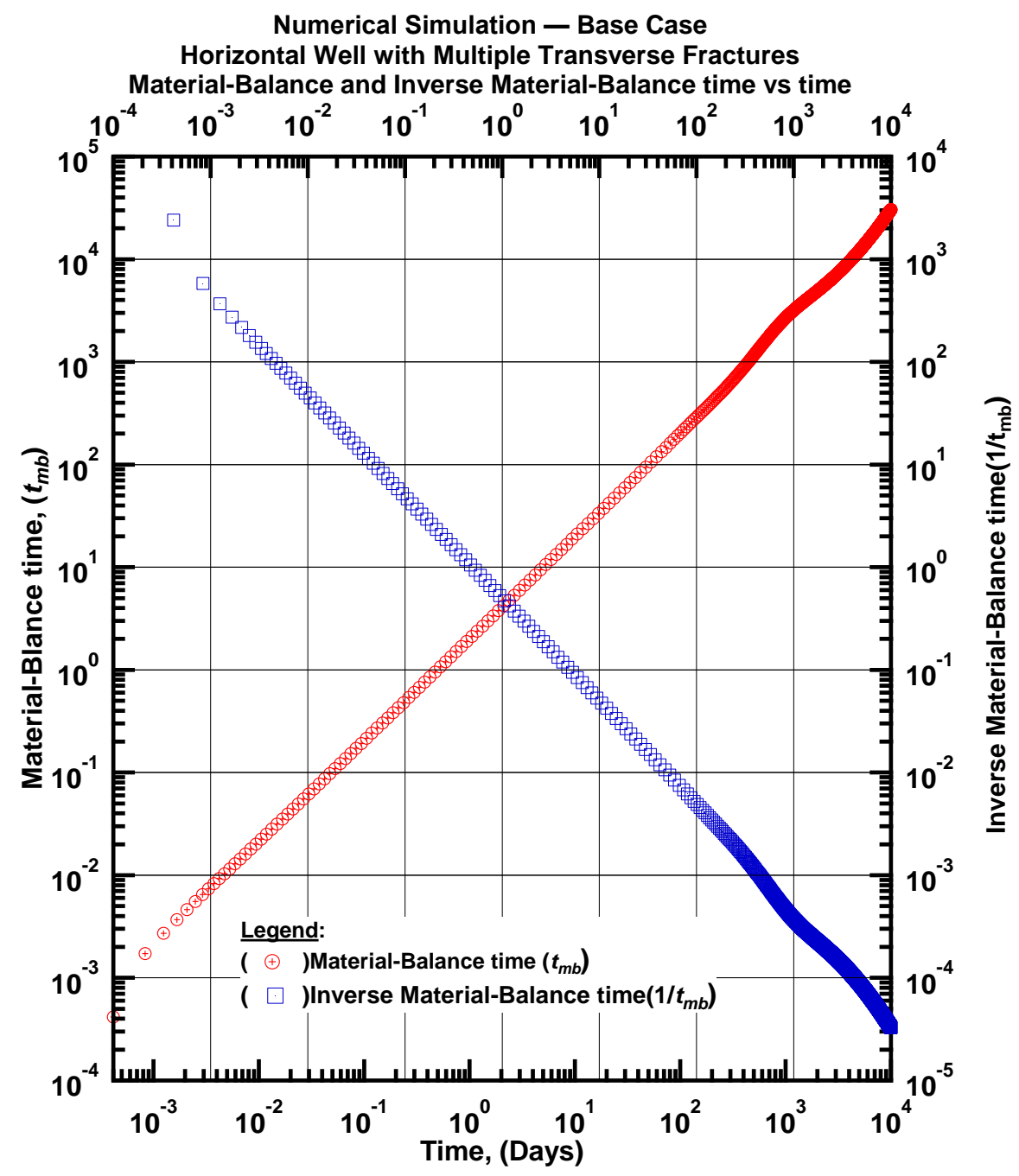

Figure D-1 - (Log-log Plot): Material-Balance tome characteristic plots for Numerical Simulation Case - material-balance time,(Days) and inverse material-balance time, (1/Days) versus production time,(Days) 
Numerical Simulation Case: Synthetic low permeability well

Next, we determine Modified Wiorkowski Model parameters from historical production data directly. This will enable us to determine EURs by analyzing character of historical data, thereby, reducing uncertainty and non-uniqueness in EUR estimation.

A plot of $\frac{d t_{m b} / d t}{t_{m b}}$ vs $\frac{1}{t_{m b}}$ or $D_{c}(t)$ gives $1 / \tilde{n}$ as slope and $\widetilde{D}_{i}$ as intercept. With these two parameters from the specialized plot and $\tilde{a}$ being equal to unity for this case, we can visually match historical timerate and time-cumulative production data with Modified Wiorkowski Model by adjusting EUR values as given in Table D-1 It is recommended to get best match by visual inspection of both time-rate and timecumulative matches.

Fig. D-2 gives a plot of $\frac{d t_{m b} / d t}{t_{m b}}$ vs $\frac{1}{t_{m b}}$ or $D_{c}(t)$. Calculations for Slope and y-intercept are also shown in Fig. D-2. For this plot we have edited the data to remove any points corresponding to time less than 1 day.

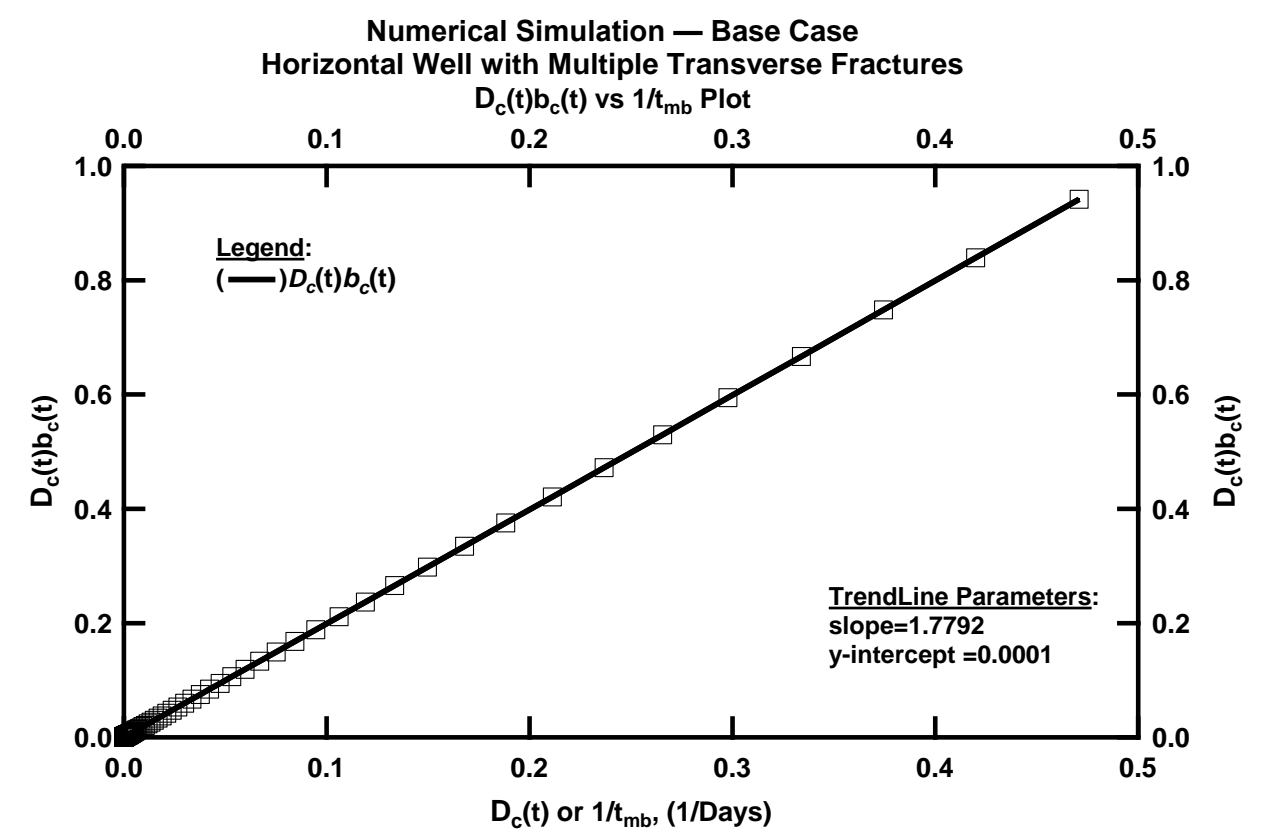

Figure D-2 - (Cartesian Plot) Estimation of parameters $\widetilde{D}_{i}$ and $\tilde{n}$ of Modified Wiorkowski Model for Numerical Simulation Case $-D_{c}(t) b_{c}(t)$ versus $D_{c}(t)$, (1/Days) 
The matched parameters are:

Table D-1 — Modified Wiorkowski Model matched parameters for Numerical Simulation case

\begin{tabular}{cc} 
Parameter & Simulation Case \\
\hline$\tilde{G}_{p}$ & $2.60 \mathrm{E}+06$ \\
$\tilde{a}$ & 0.999 \\
$\tilde{D}_{i}$ & 0.0001 \\
$\tilde{n}$ & 0.56 \\
\hline
\end{tabular}

For numerical simulation case, we used the above described technique to estimate $E U R$. 


\section{APPENDIX E}

\section{INVENTORY OF $q D b$ PLOTS FOR THIS WORK}

We present the $q D b$ analysis plots for the Modified Wiorkowski Model and Modified Ilk Model ratedecline models.

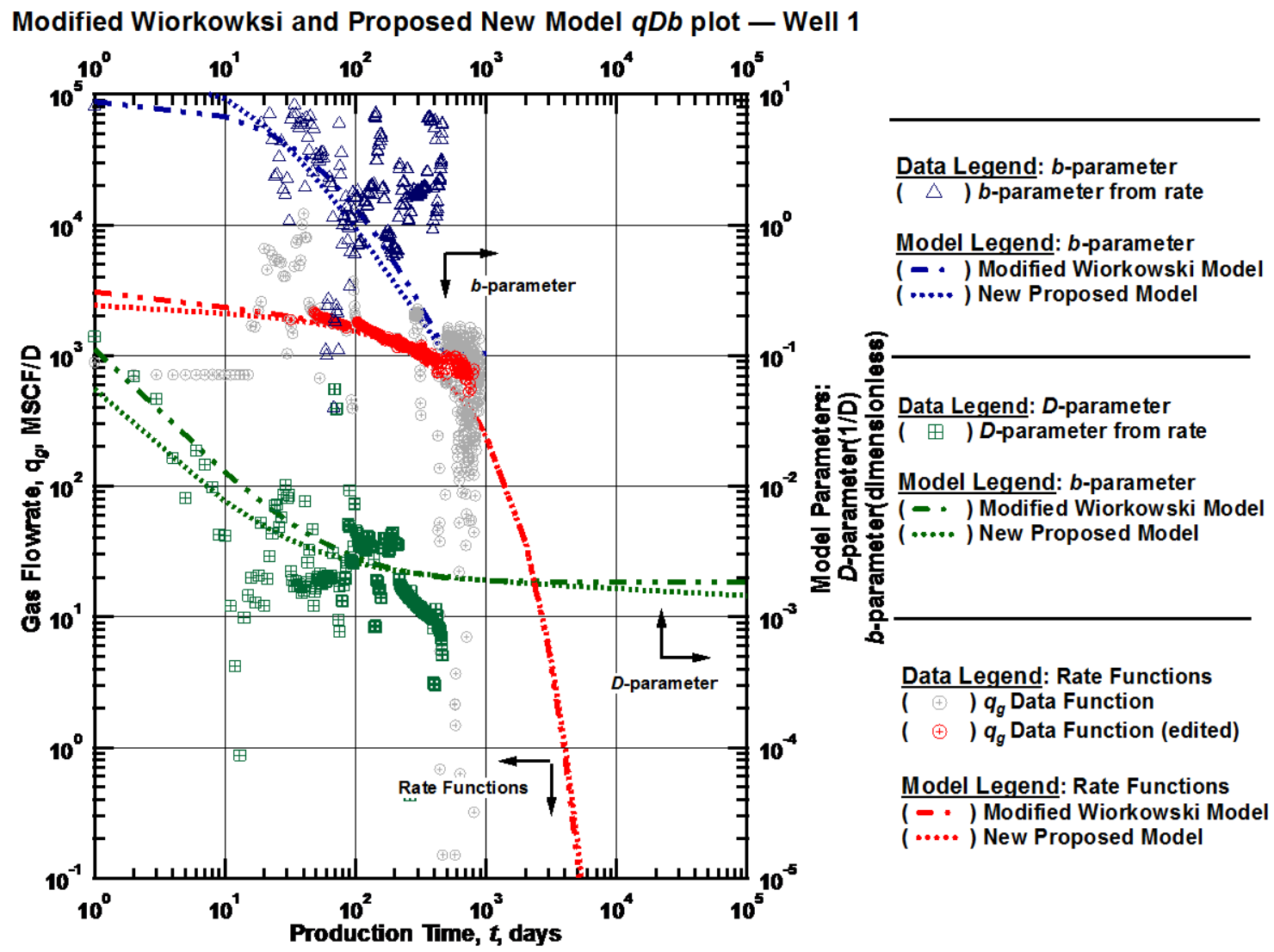

Figure E-1 _ $\quad$ (Log-log Plot): $q D b$ plot — gas flow rate $\left(q_{g i}\right), D$ - and $b$-parameters versus production time and Modified Wiorkowski Model and Modified Ilk Model matches for Well 1. 


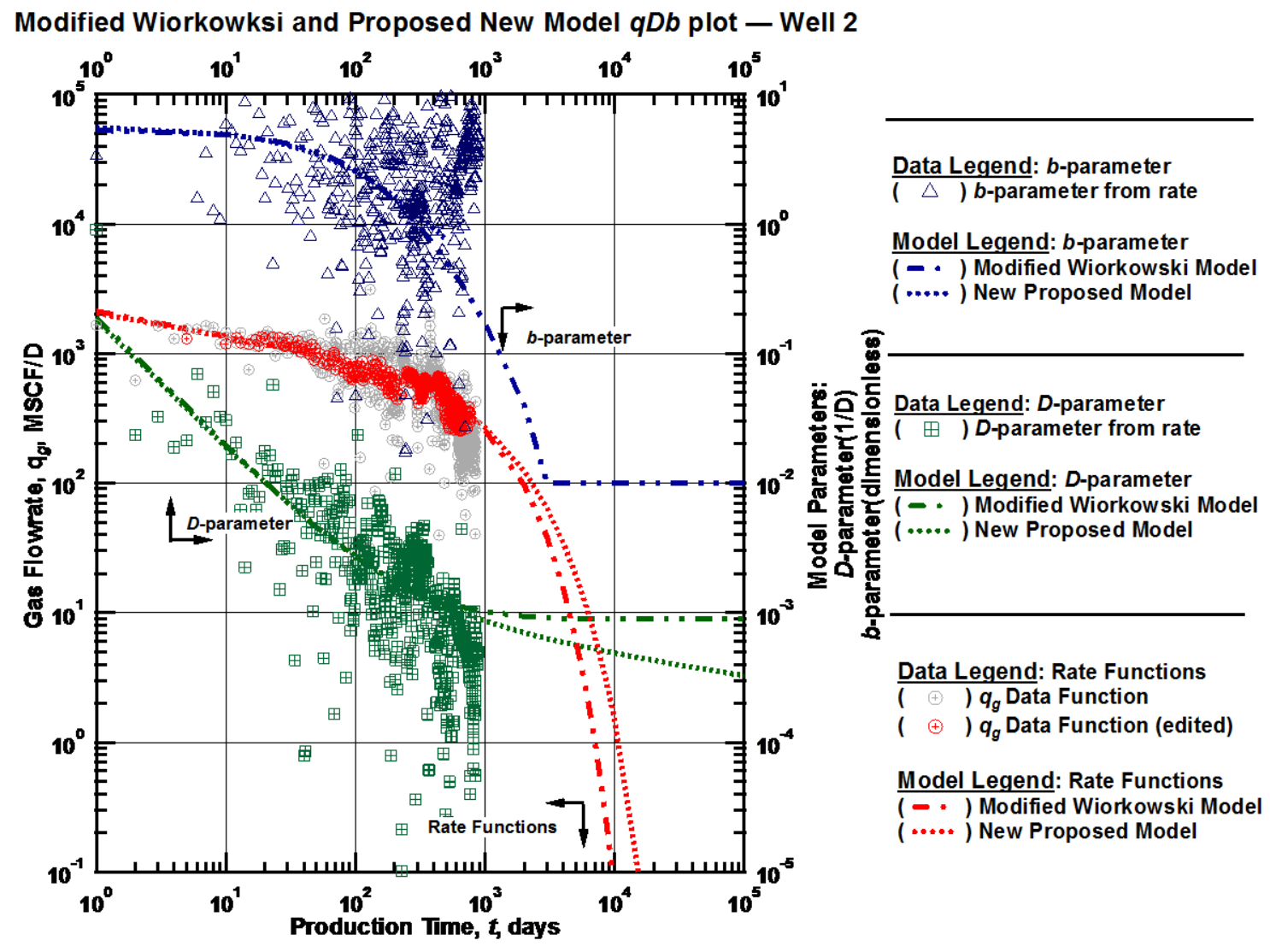

Figure E-2 - (Log-log Plot): $q D b$ plot - gas flow rate $\left(q_{g i}\right), D$ - and $b$-parameters versus production time and Modified Wiorkowski Model and Modified Ilk Model matches for Well 2. 


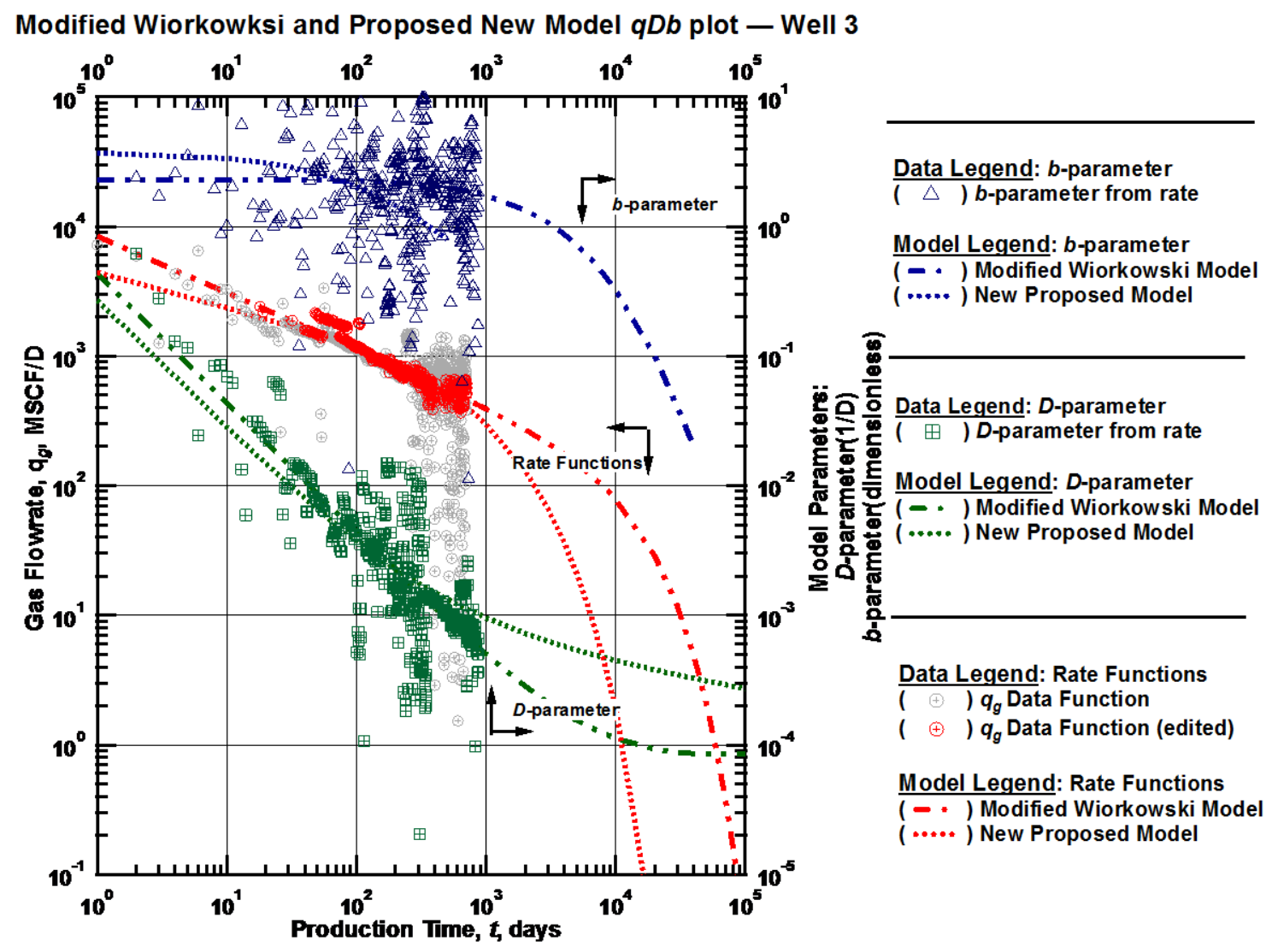

Figure E- 3 - (Log-log Plot): $q D b$ plot - gas flow rate $\left(q_{g i}\right), D$ - and $b$-parameters versus production time and Modified Wiorkowski Model and Modified Ilk Model matches for Well 3. 


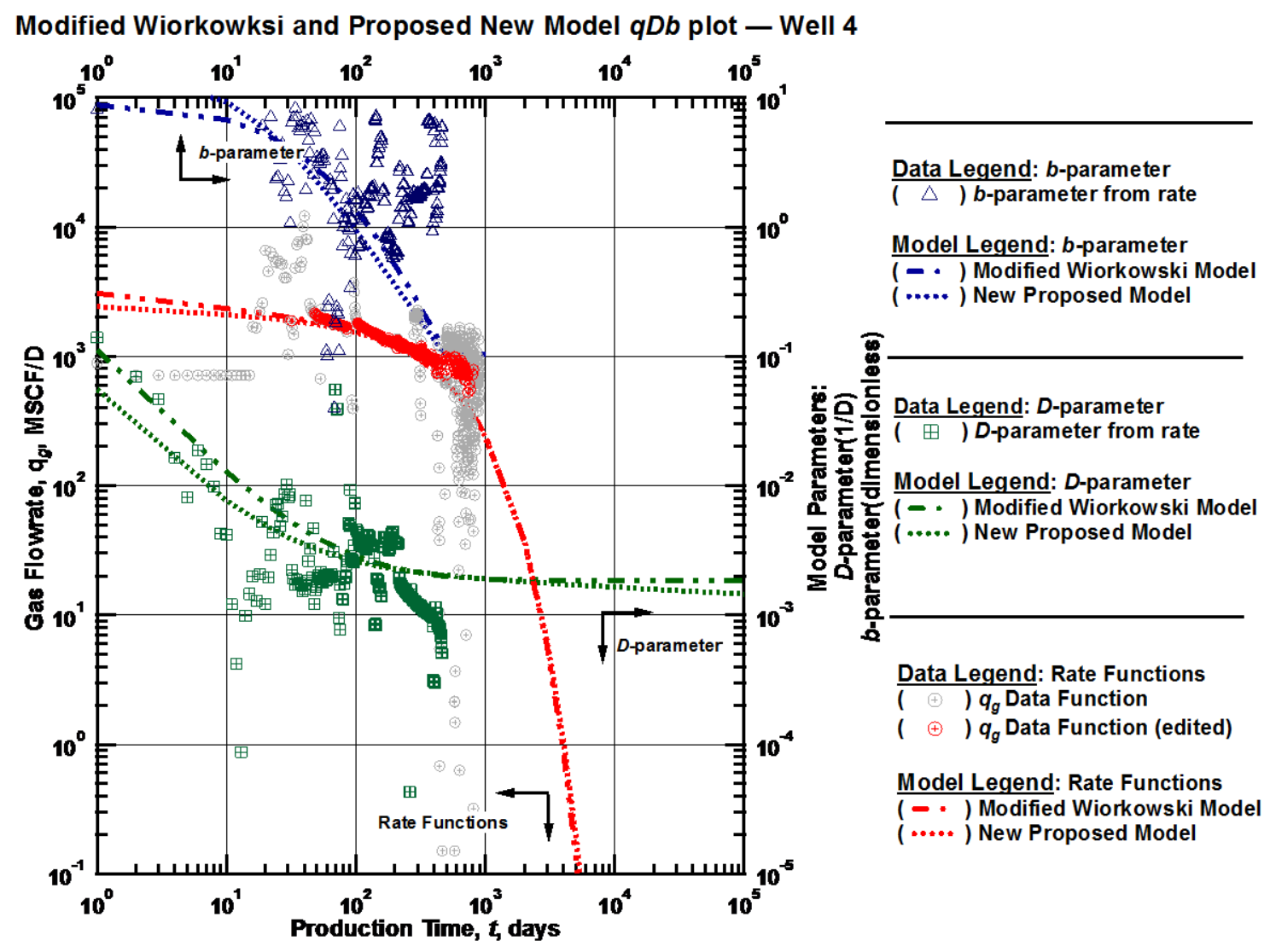

Figure E-4 - (Log-log Plot): $q D b$ plot - gas flow rate $\left(q_{g i}\right), D$ - and $b$-parameters versus production time and Modified Wiorkowski Model and Modified Ilk Model matches for Well 4. 


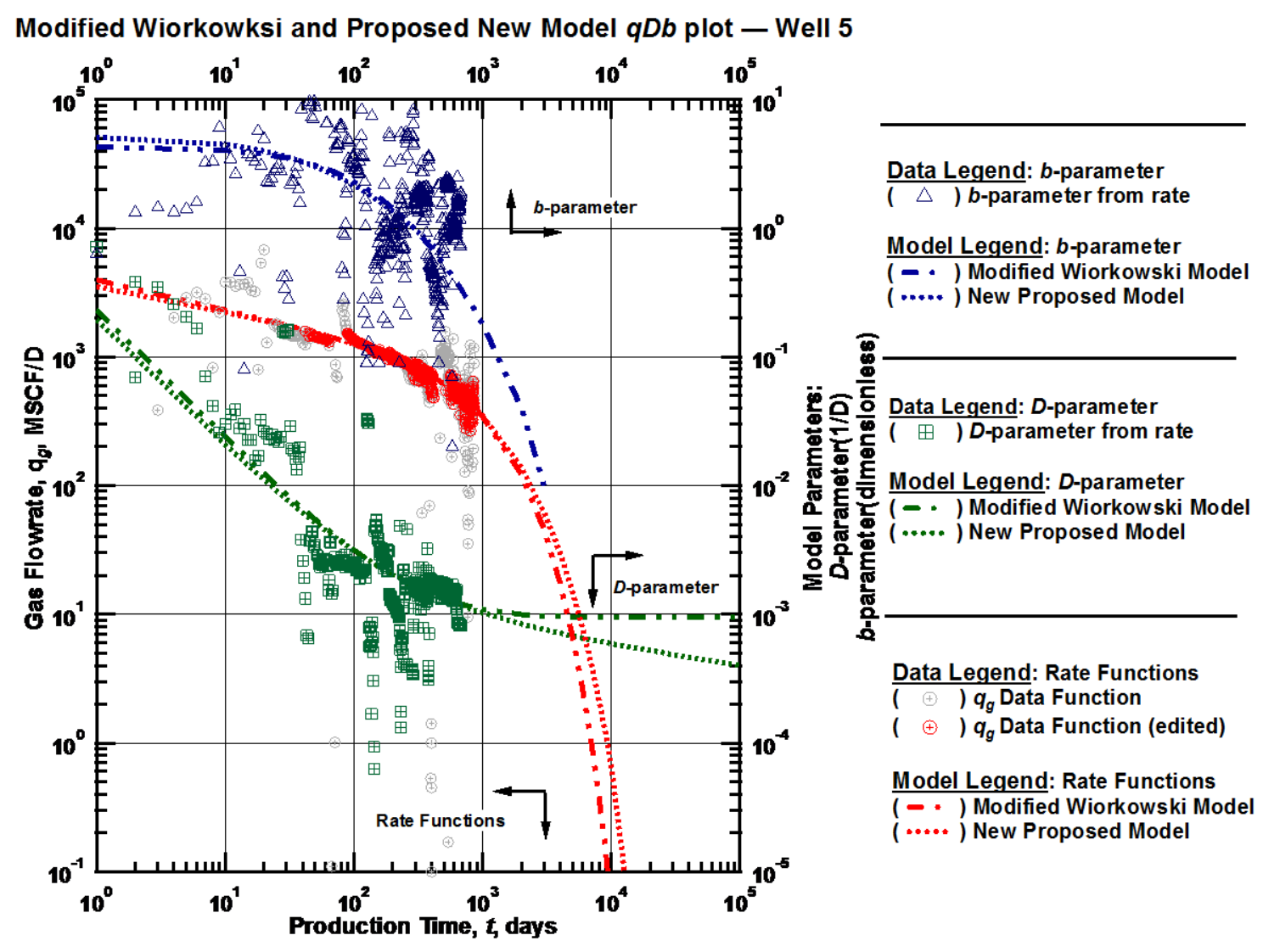

Figure E-5 - (Log-log Plot): $q D b$ plot - gas flow rate $\left(q_{g i}\right), D$ - and $b$-parameters versus production time and Modified Wiorkowski Model and Modified Ilk Model matches for Well 5. 


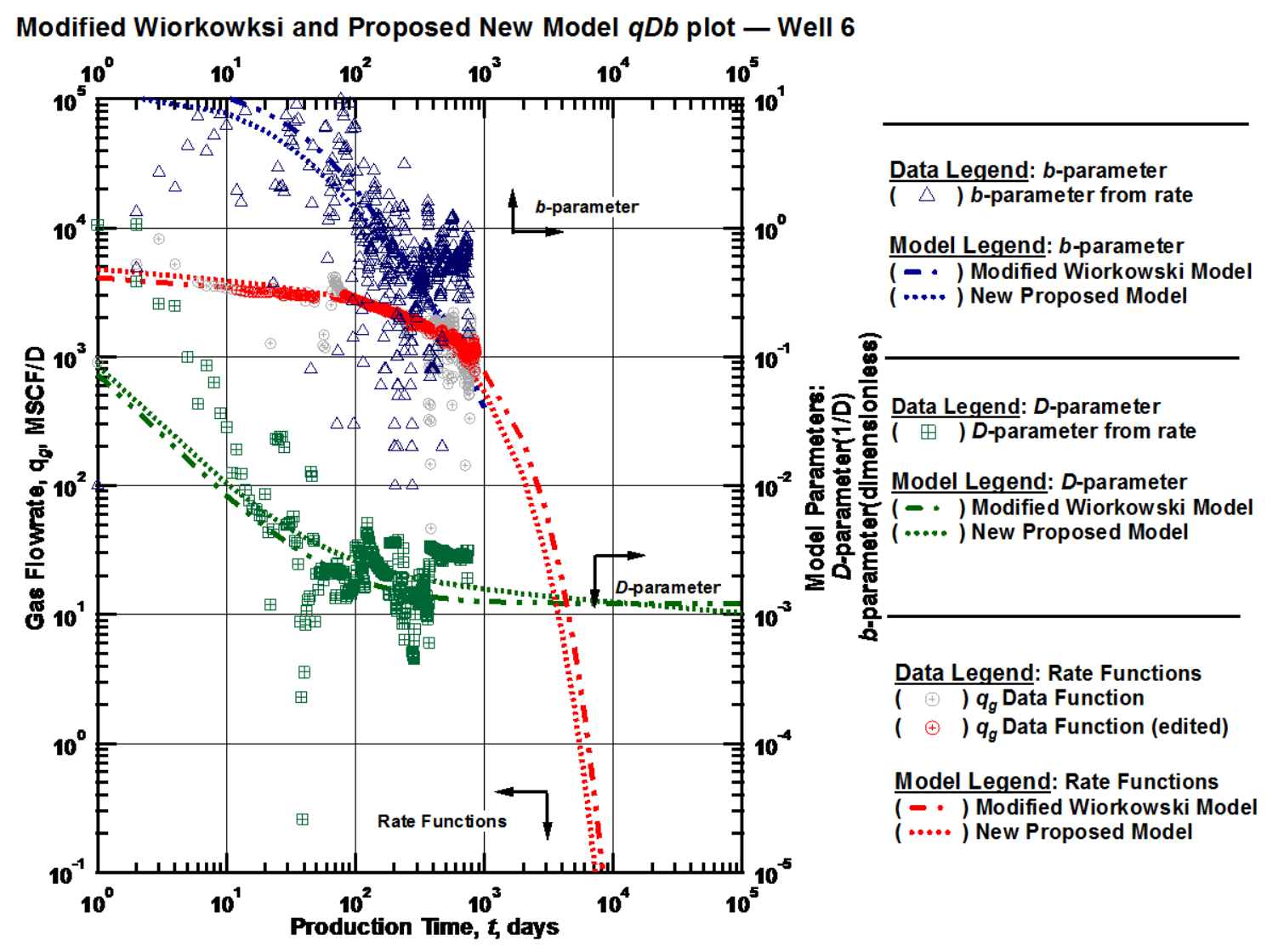

Figure E-6 - (Log-log Plot): $q D b$ plot - gas flow rate $\left(q_{g i}\right), D$ - and $b$-parameters versus production time and Modified Wiorkowski Model and Modified Ilk Model matches for Well 6. 


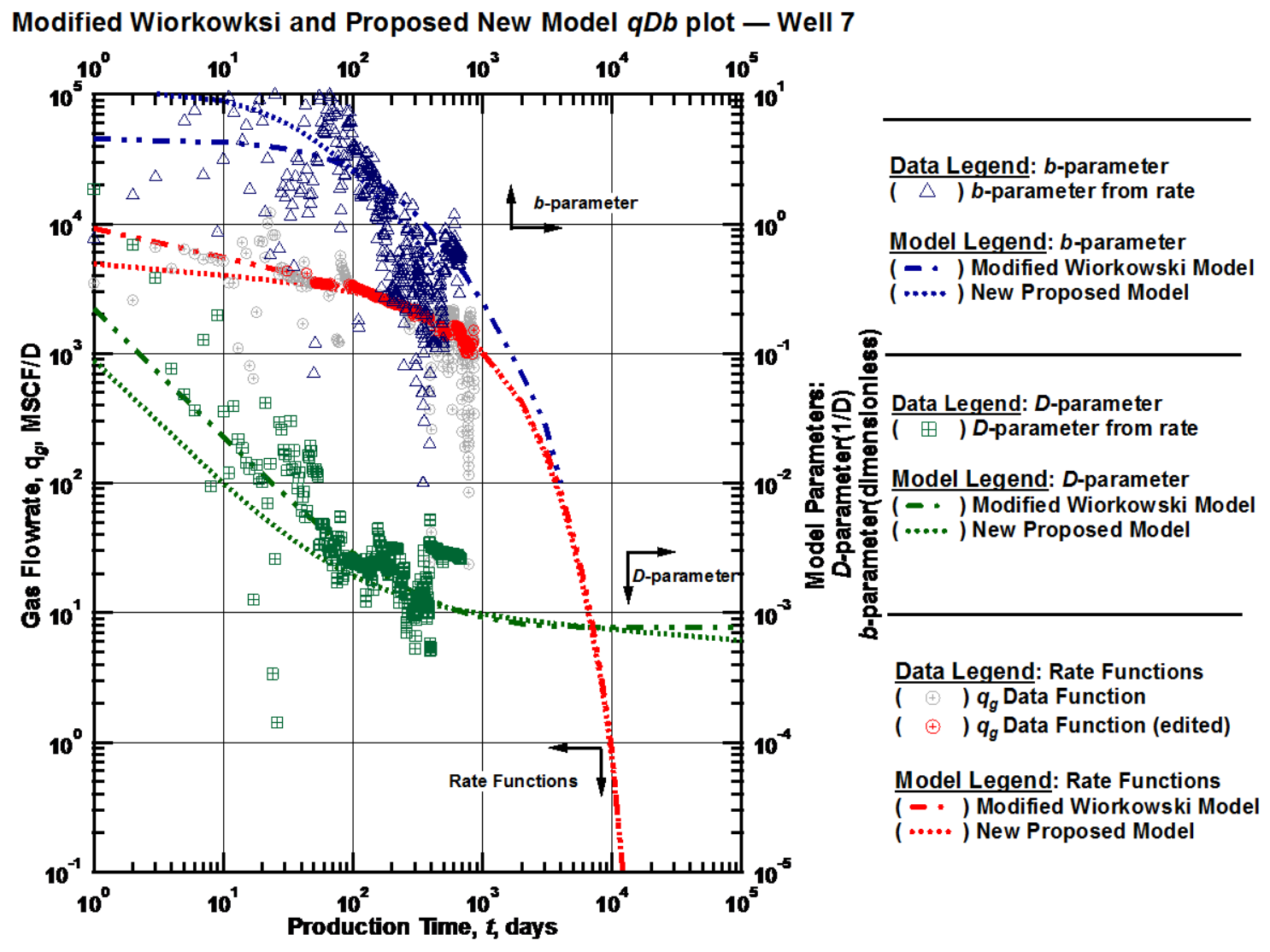

Figure E-7 - (Log-log Plot): $q D b$ plot - gas flow rate $\left(q_{g i}\right), D$ - and $b$-parameters versus production time and Modified Wiorkowski Model and Modified Ilk Model matches for Well 7. 


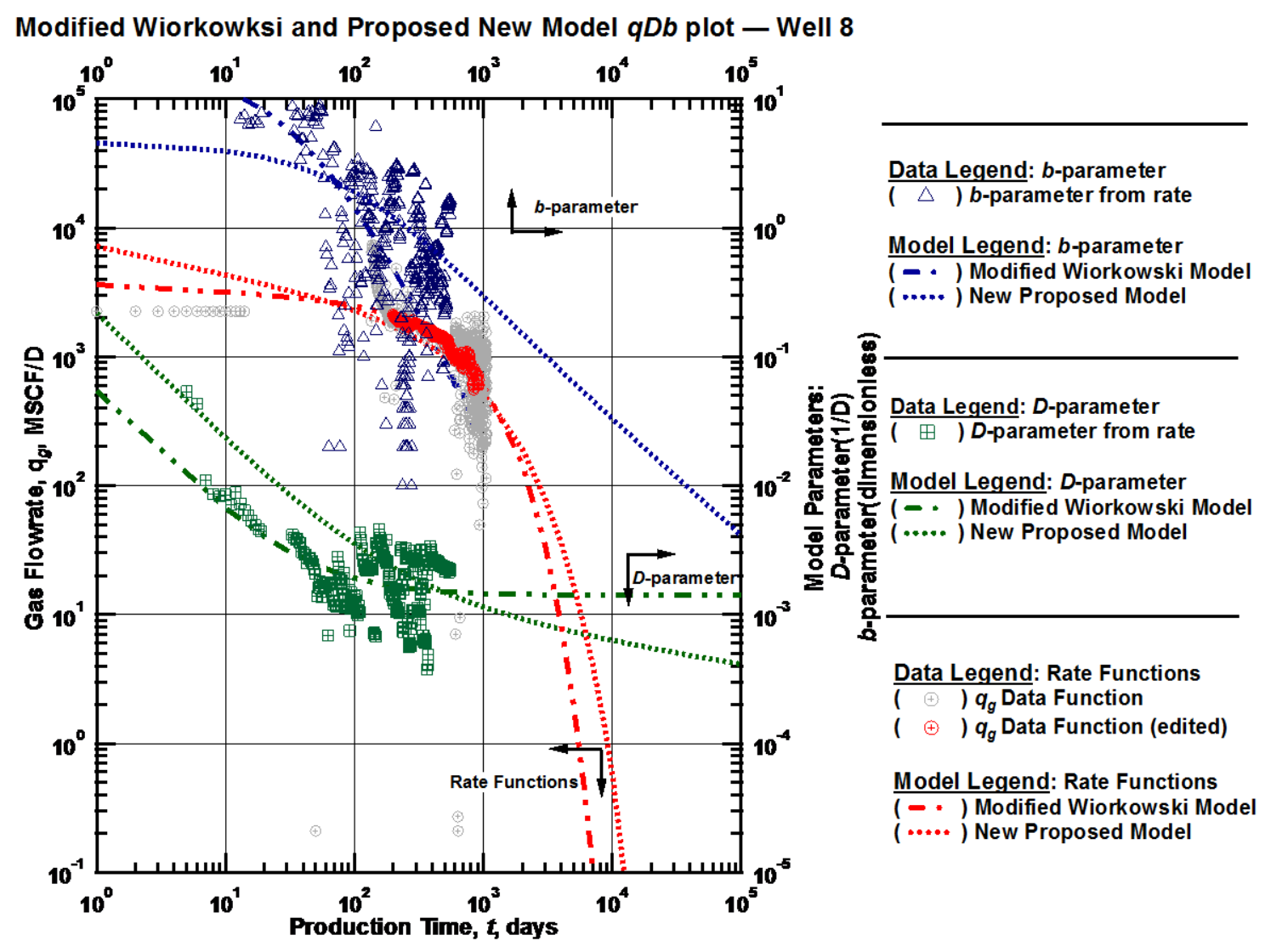

Figure E-8 - (Log-log Plot): $q D b$ plot - gas flow rate $\left(q_{g i}\right), D$ - and $b$-parameters versus production time and Modified Wiorkowski Model and Modified Ilk Model matches for Well 8. 


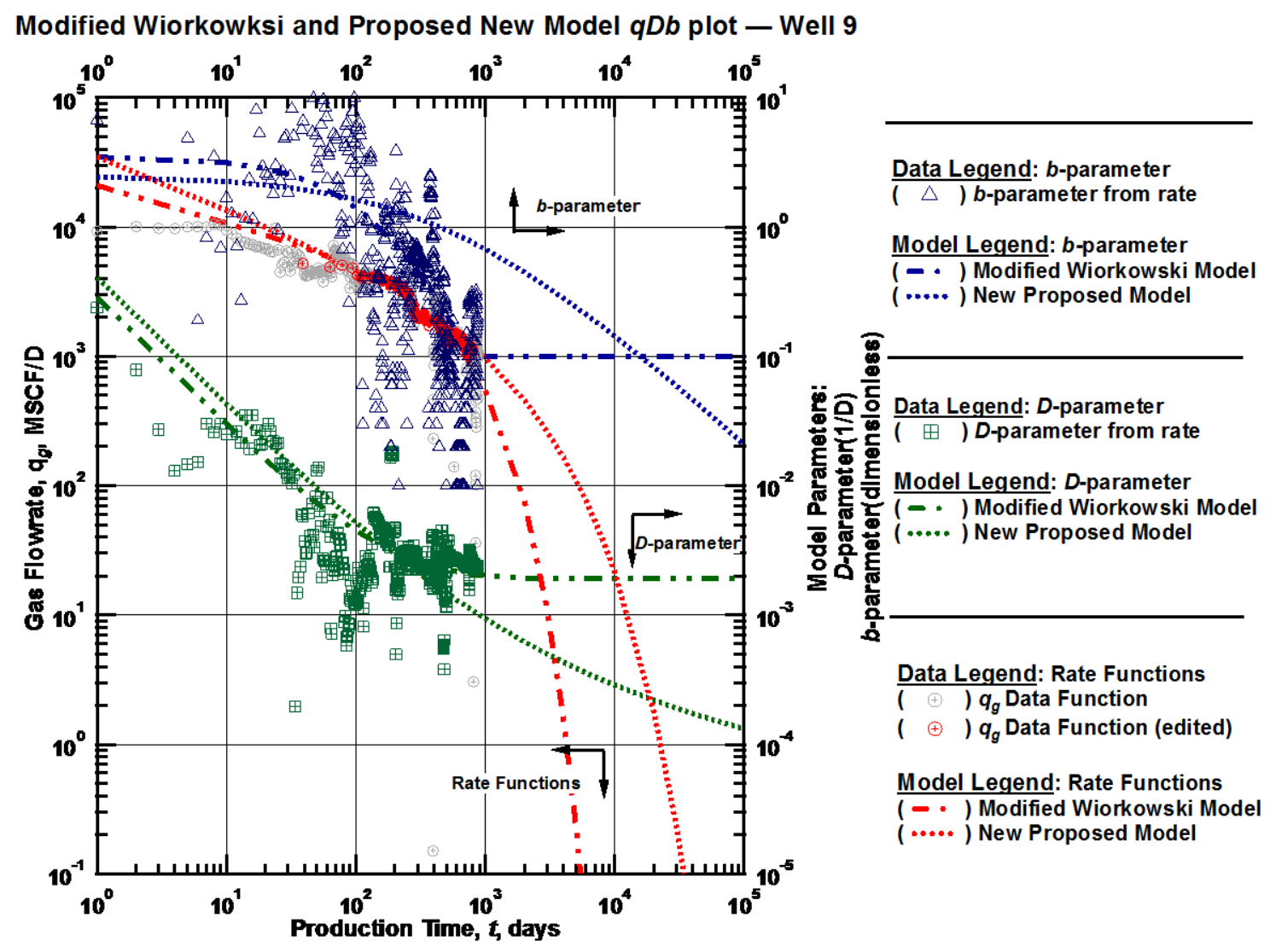

Figure E-9 - (Log-log Plot): $q D b$ plot - gas flow rate $\left(q_{g i}\right), D$ - and $b$-parameters versus production time and Modified Wiorkowski Model and Modified Ilk Model matches for Well 9. 


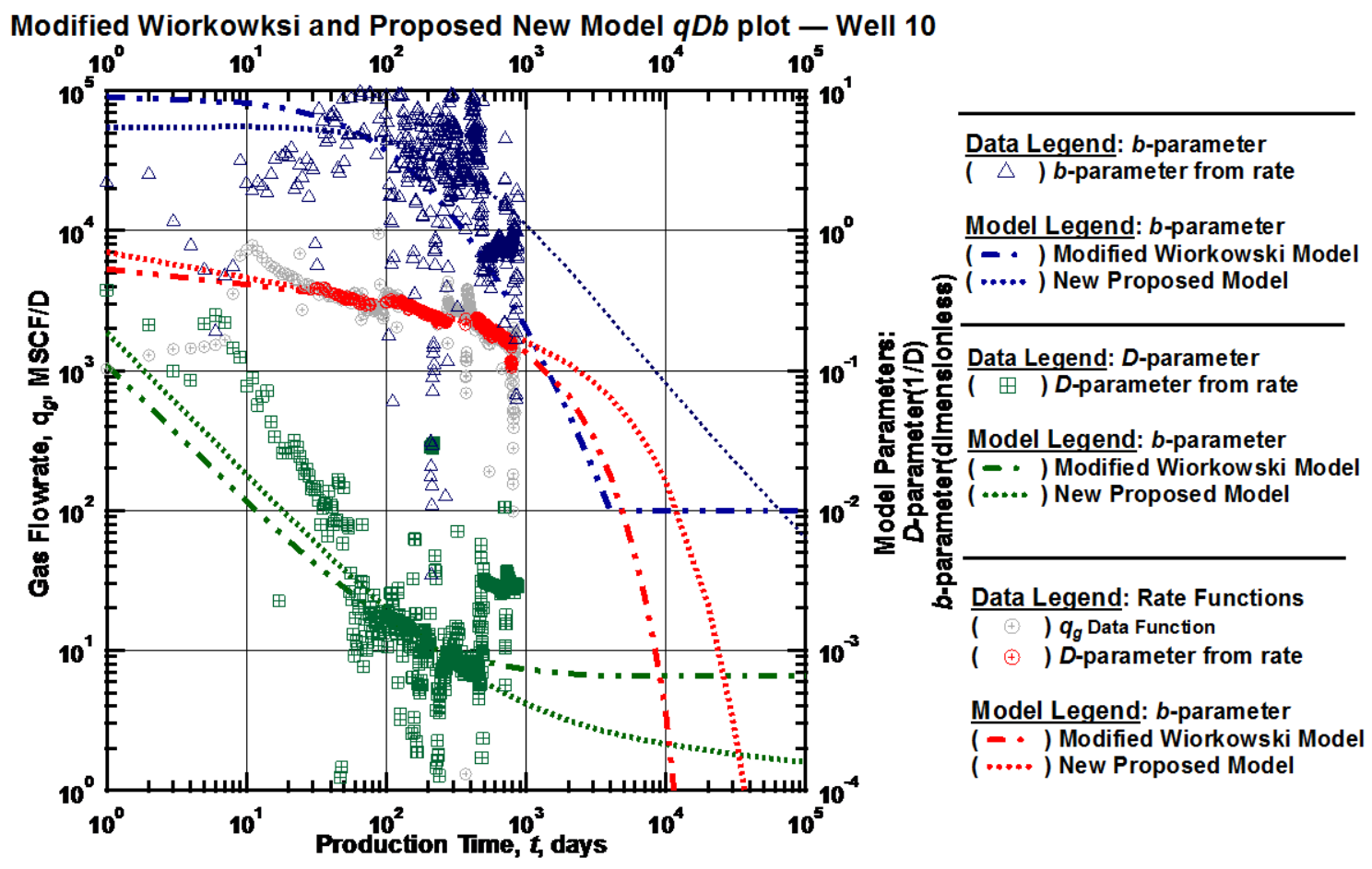

Figure E-10 - (Log-log Plot): $q D b$ plot — gas flow rate $\left(q_{g i}\right), D$ - and $b$-parameters versus production time and Modified Wiorkowski Model and Modified Ilk Model matches for Well 10. 
Modified Wiorkowksi and Proposed New Model qDb plot - Well 11

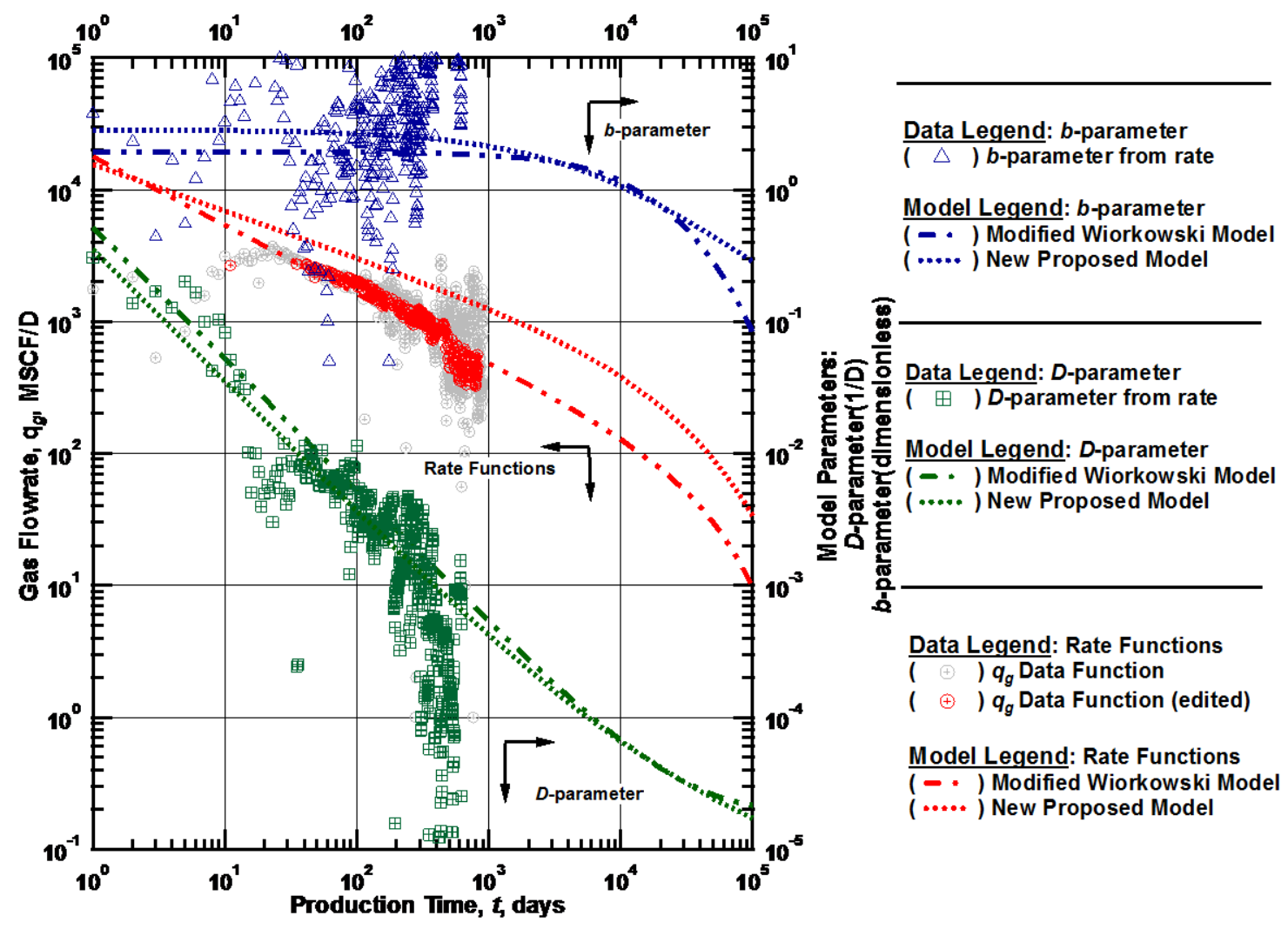

Figure E-11 - (Log-log Plot): $q D b$ plot — gas flow rate $\left(q_{g i}\right), D$ - and $b$-parameters versus production time and Modified Wiorkowski Model and Modified Ilk Model matches for Well 11. 
Modified Wiorkowksi and Proposed New Model qDb plot - Well 12

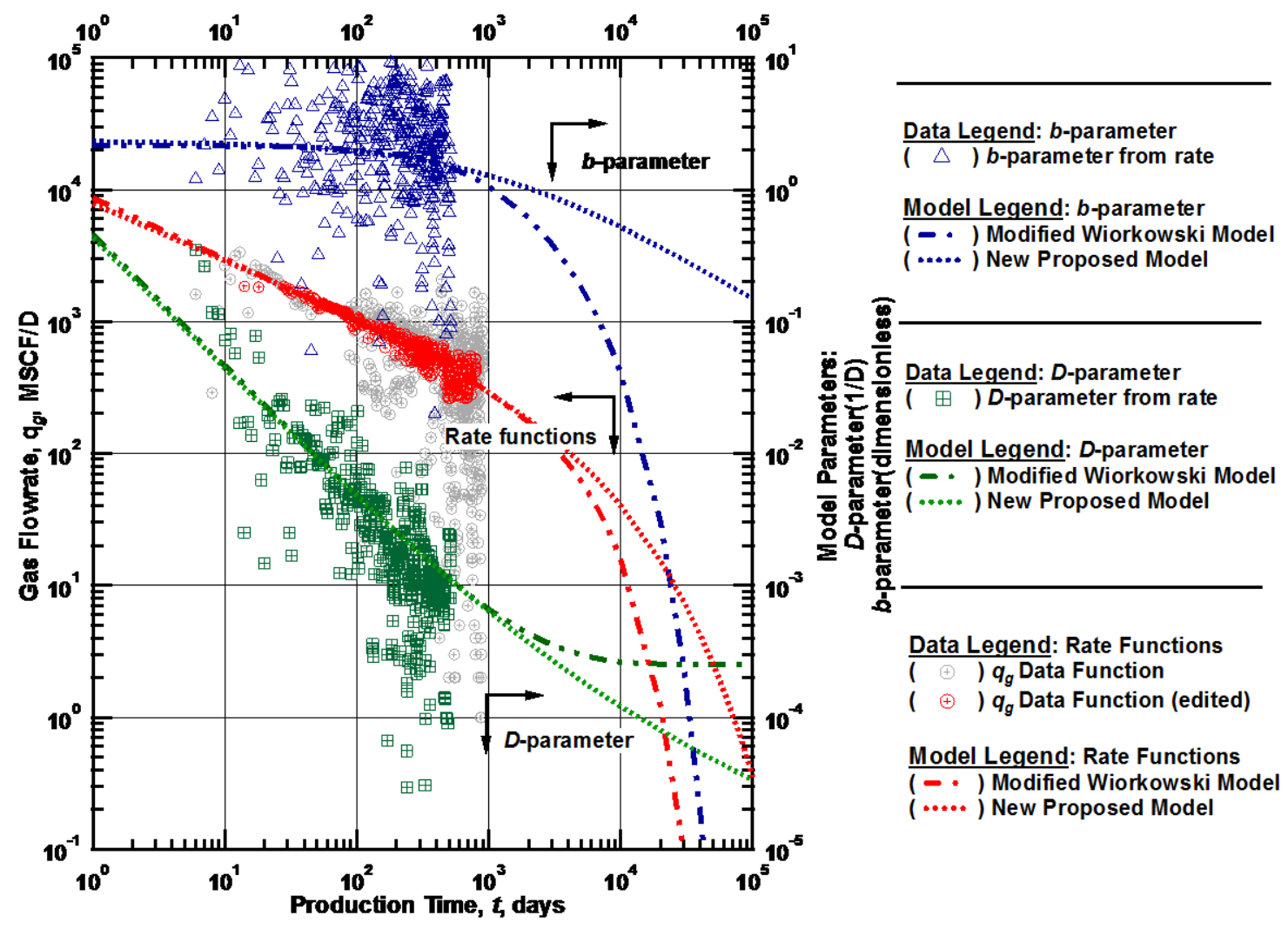

Figure E-12 - (Log-log Plot): $q D b$ plot - gas flow rate $\left(q_{g i}\right), D$ - and $b$-parameters versus production time and Modified Wiorkowski Model and Modified Ilk Model matches for Well 12. 
Modified Wiorkowksi and Proposed New Model qDb plot - Well 13

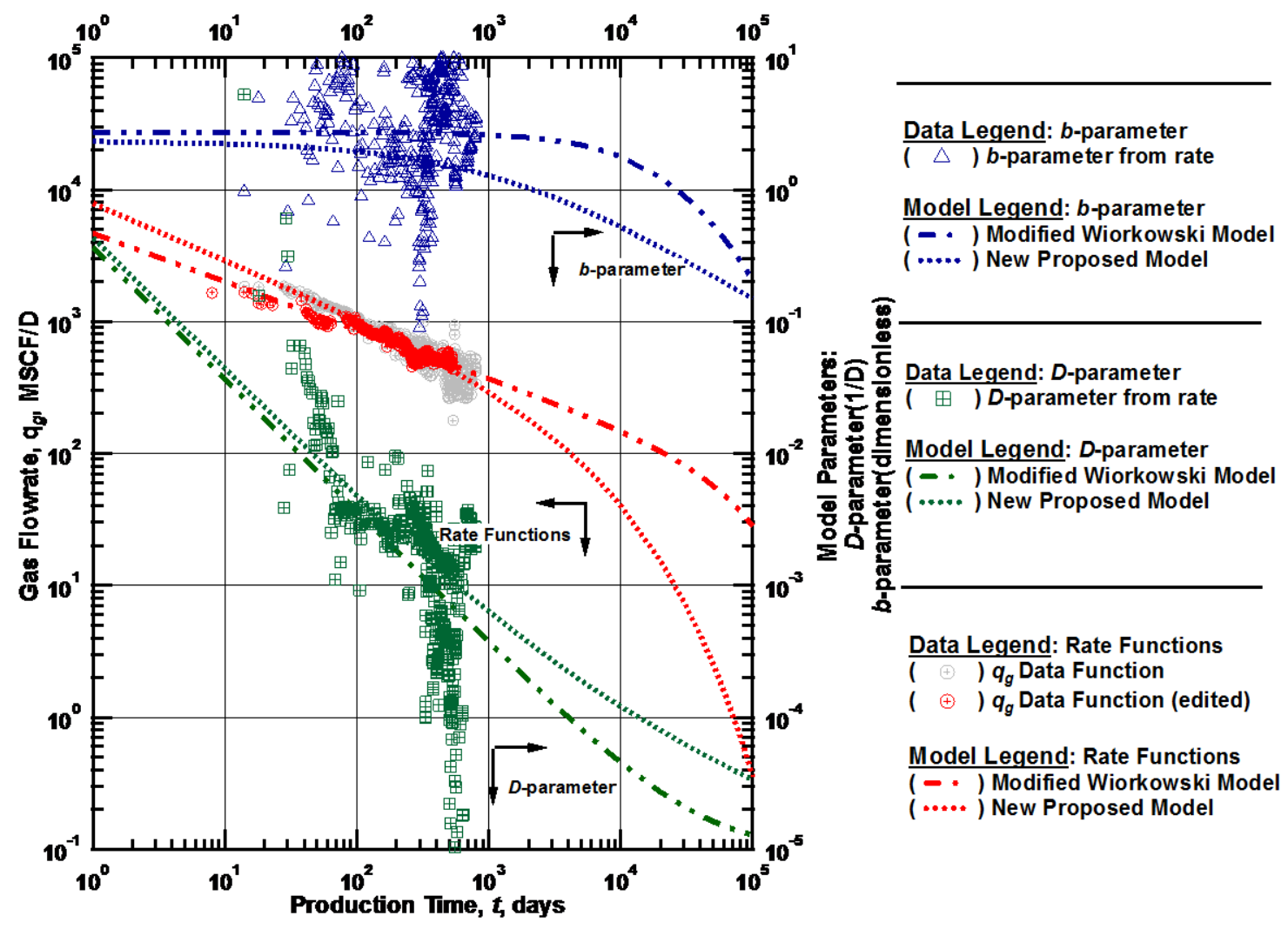

Figure E-13 - (Log-log Plot): $q D b$ plot - gas flow rate $\left(q_{g i}\right), D$ - and $b$-parameters versus production time and Modified Wiorkowski Model and Modified Ilk Model matches for Well 13 
Modified Wiorkowksi and Proposed New Model qDb plot - Well 14

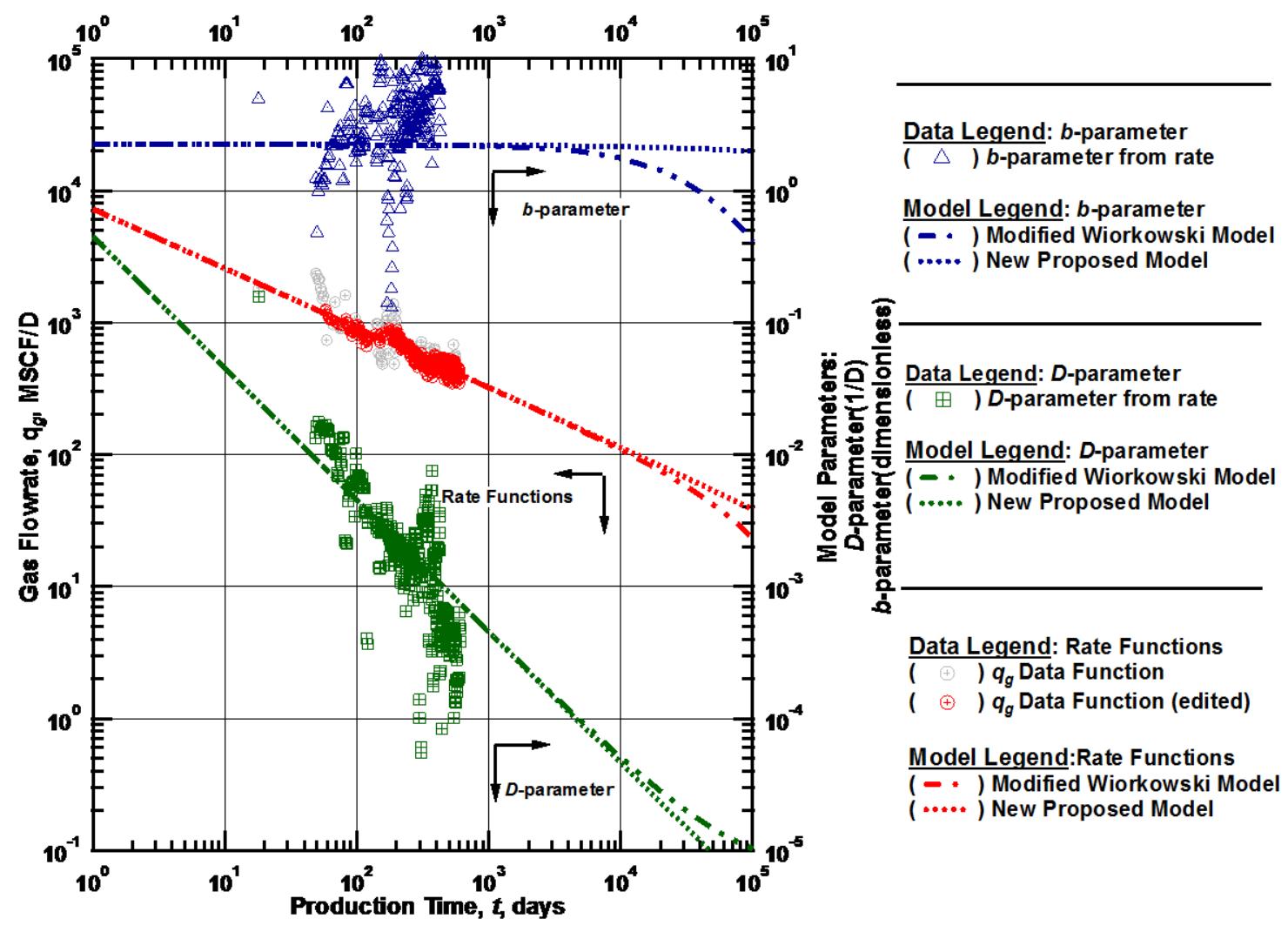

Figure E-14 - (Log-log Plot): $q D b$ plot - gas flow rate $\left(q_{g i}\right), D$ - and $b$-parameters versus production time and Modified Wiorkowski Model and Modified Ilk Model matches for Well 14. 
Modified Wiorkowksi and Proposed New Model qDb plot - Well 15

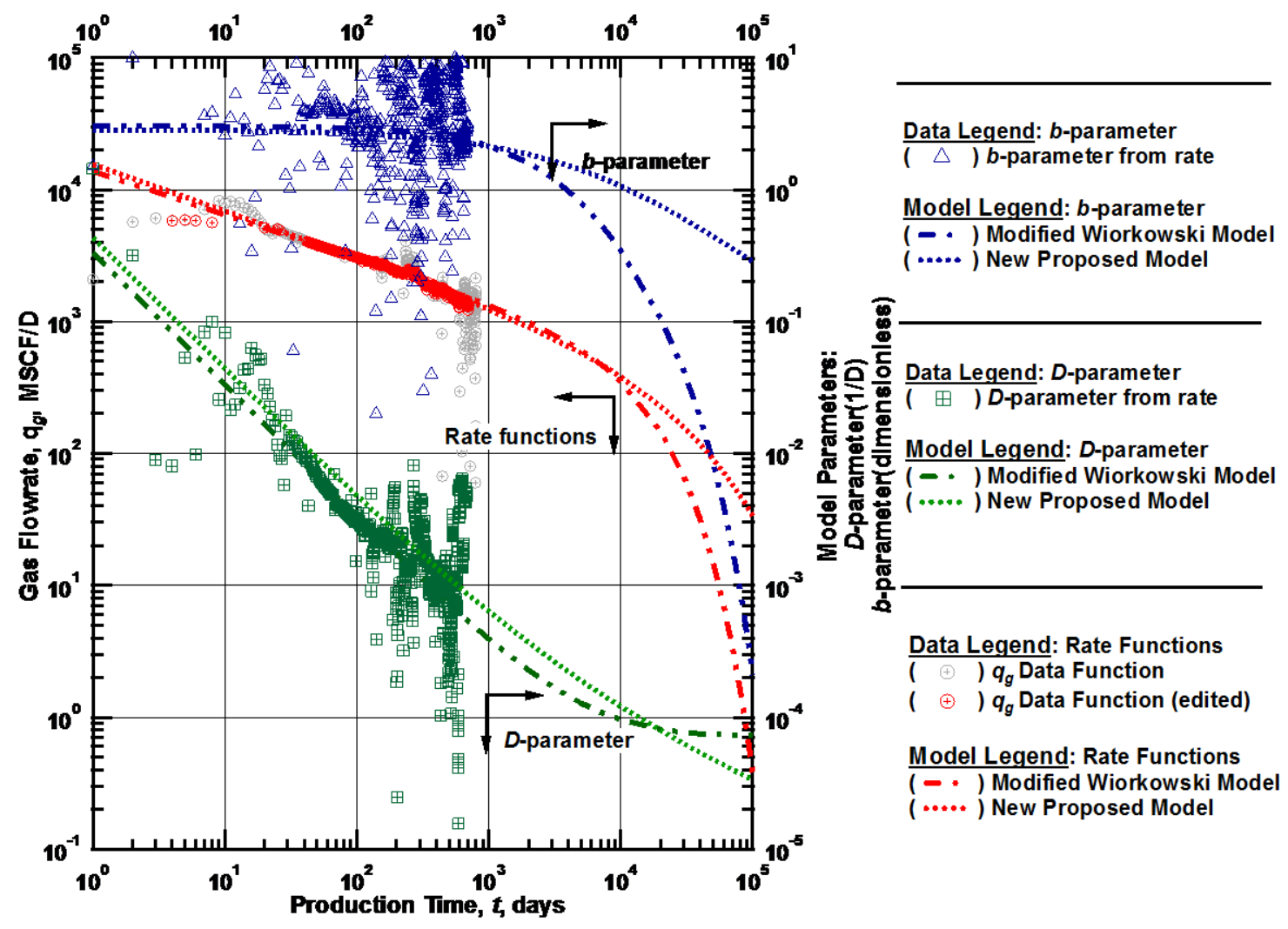

Figure E-15 - (Log-log Plot): $q D b$ plot - gas flow rate $\left(q_{g i}\right), D$ - and $b$-parameters versus production time and Modified Wiorkowski Model and Modified Ilk Model matches for Well 15. 
Modified Wiorkowksi and Proposed New Model qDb plot - Well 16

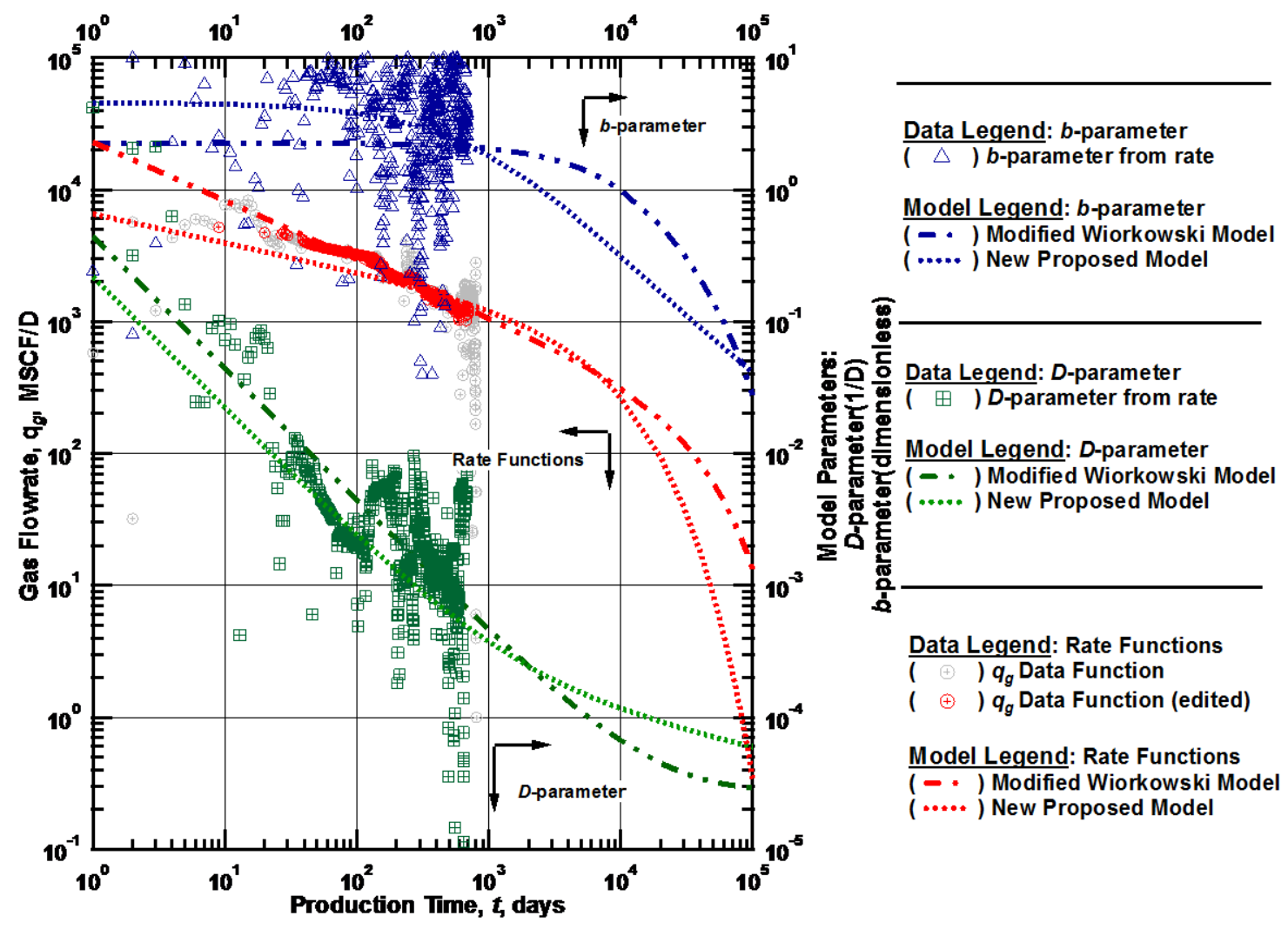

Figure E-16 - (Log-log Plot): $q D b$ plot - gas flow rate $\left(q_{g i}\right), D$ - and $b$-parameters versus production time and Modified Wiorkowski Model and Modified Ilk Model matches for Well 16. 
Modified Wiorkowksi and Proposed New Model qDb plot - Well 17

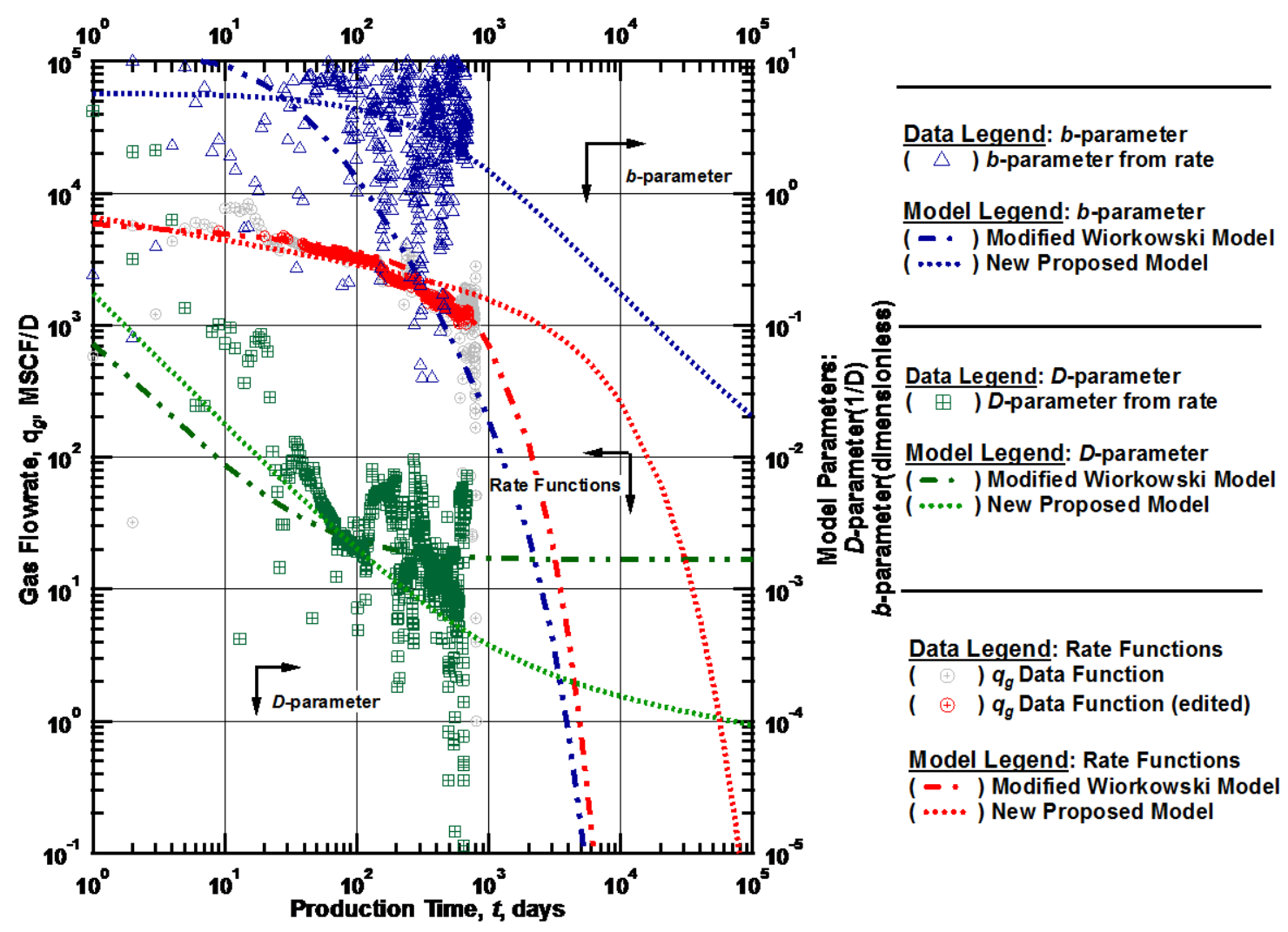

Figure E-17 - (Log-log Plot): $q D b$ plot - gas flow rate $\left(q_{g i}\right), D$ - and $b$-parameters versus production time and Modified Wiorkowski Model and Modified Ilk Model matches for Well 17. 
Modified Wiorkowksi and Proposed New Model qDb plot - Well 18

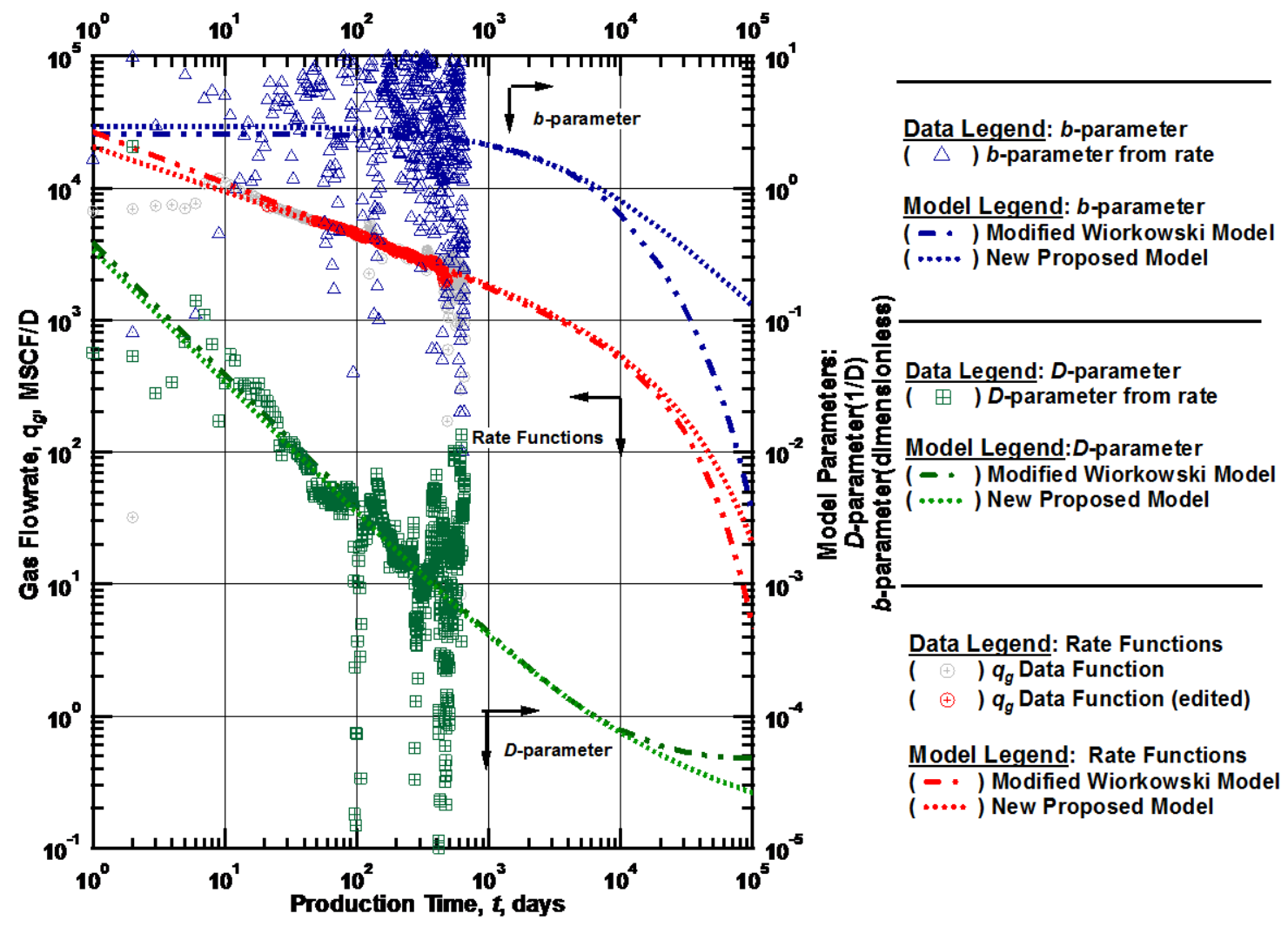

Figure E-18 - (Log-log Plot): $q D b$ plot - gas flow rate $\left(q_{g i}\right), D$ - and $b$-parameters versus production time and Modified Wiorkowski Model and Modified Ilk Model matches for Well 18. 
Modified Wiorkowksi and Proposed New Model qDb plot - Well 19

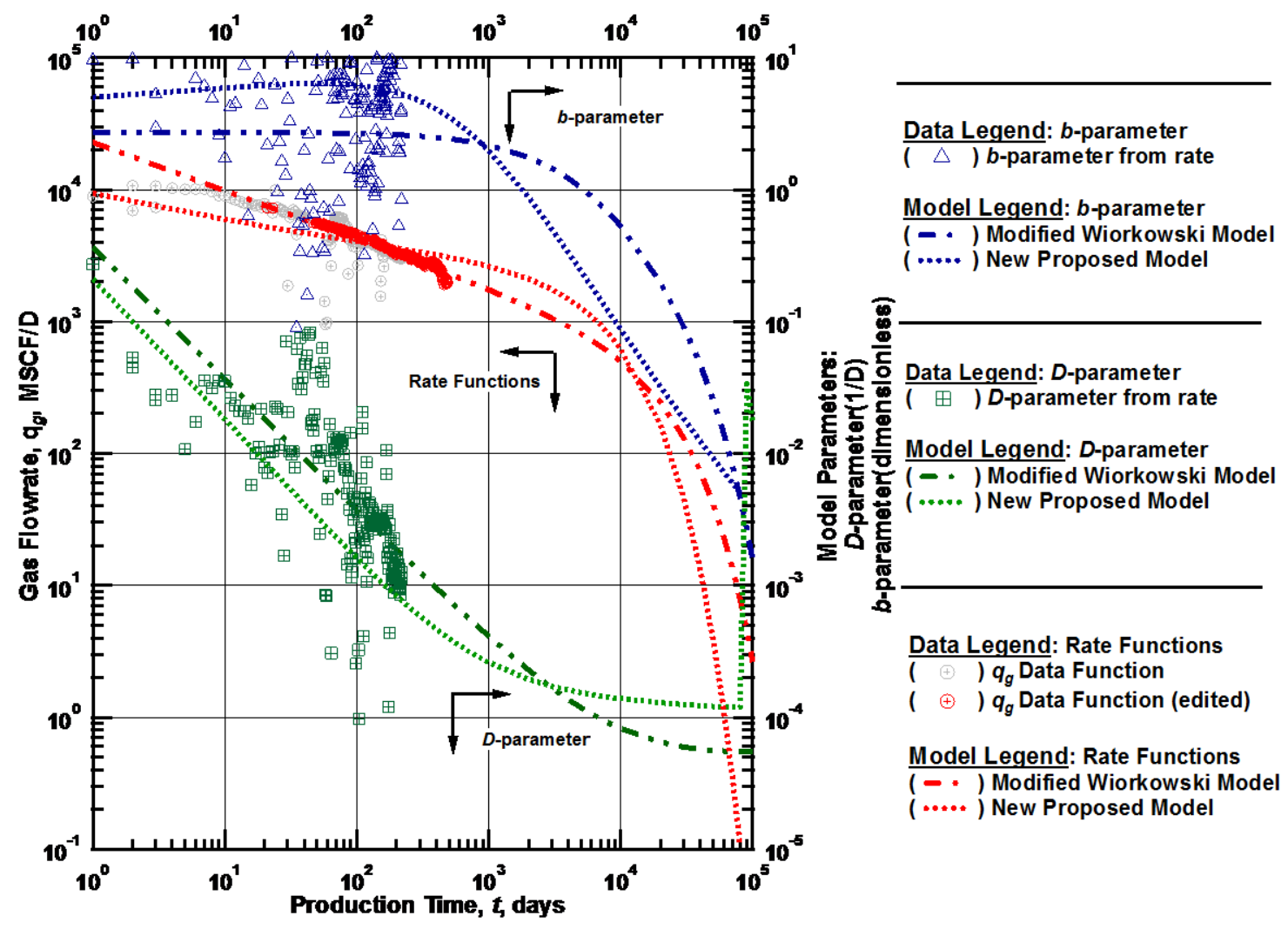

Figure E-19 - (Log-log Plot): $q D b$ plot - gas flow rate $\left(q_{g i}\right), D$ - and $b$-parameters versus production time and Modified Wiorkowski Model and Modified Ilk Model matches for Well 19. 
Modified Wiorkowksi and Proposed New Model qDb plot - Well 20

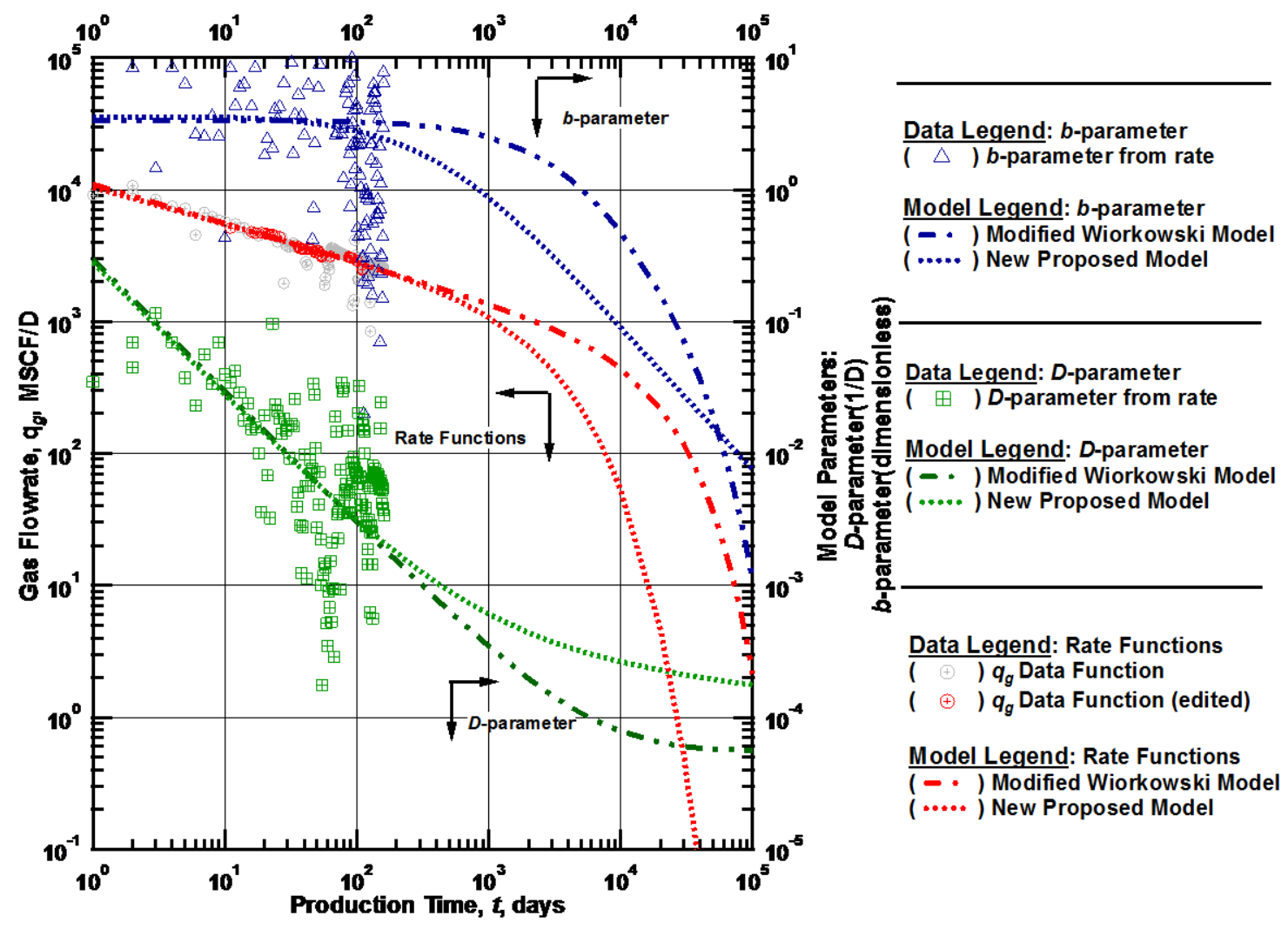

Figure E-20 - (Log-log Plot): $q D b$ plot - gas flow rate $\left(q_{g i}\right), D$ - and $b$-parameters versus production time and Modified Wiorkowski Model and Modified Ilk Model matches for Well 20. 
Modified Wiorkowksi and Proposed New Model qDb plot - Well 21

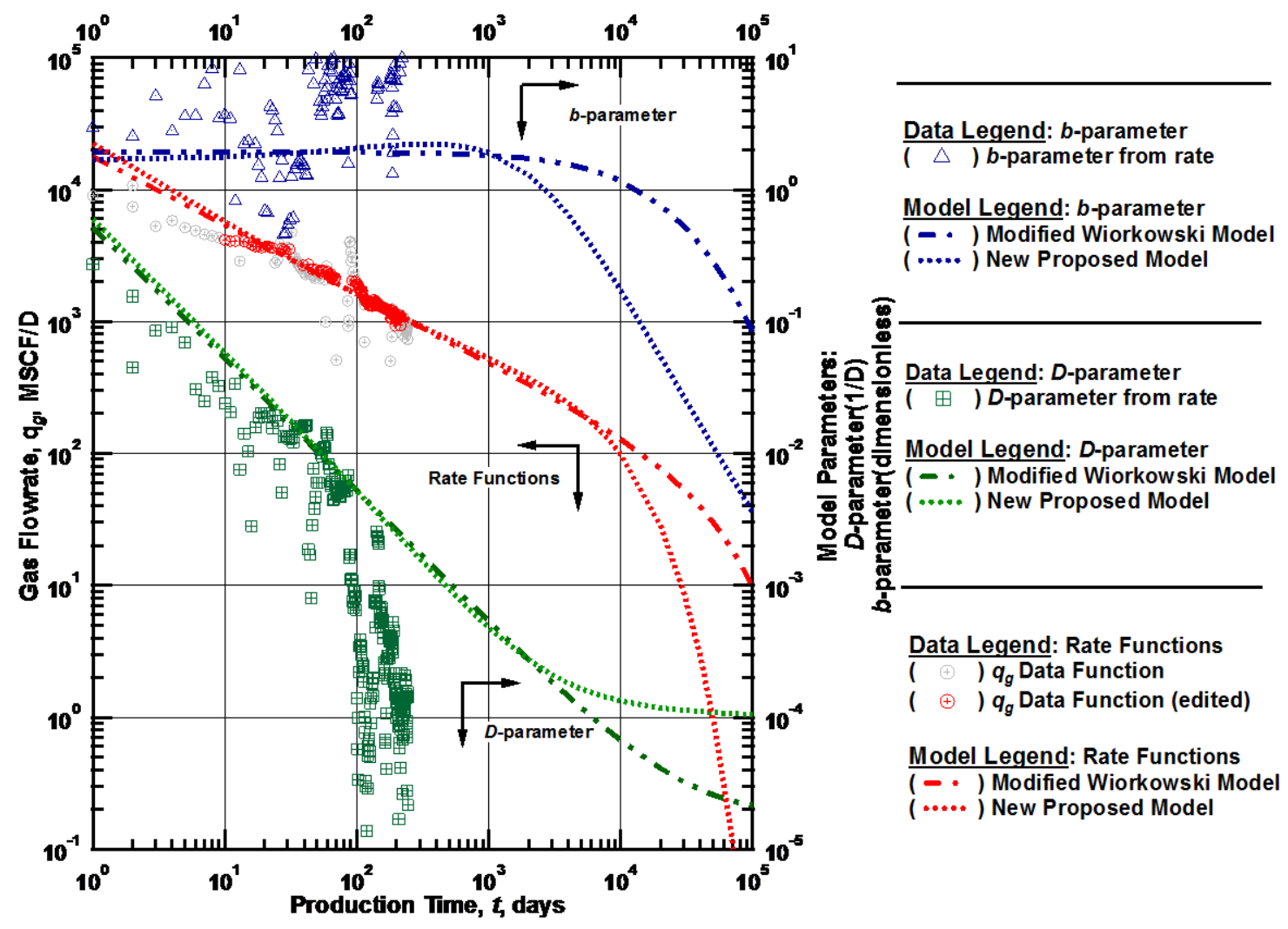

Figure E-21 - (Log-log Plot): $q D b$ plot — gas flow rate $\left(q_{g i}\right), D$ - and $b$-parameters versus production time and Modified Wiorkowski Model and Modified Ilk Model matches for Well 21. 
Modified Wiorkowksi and Proposed New Model qDb plot - Well 22

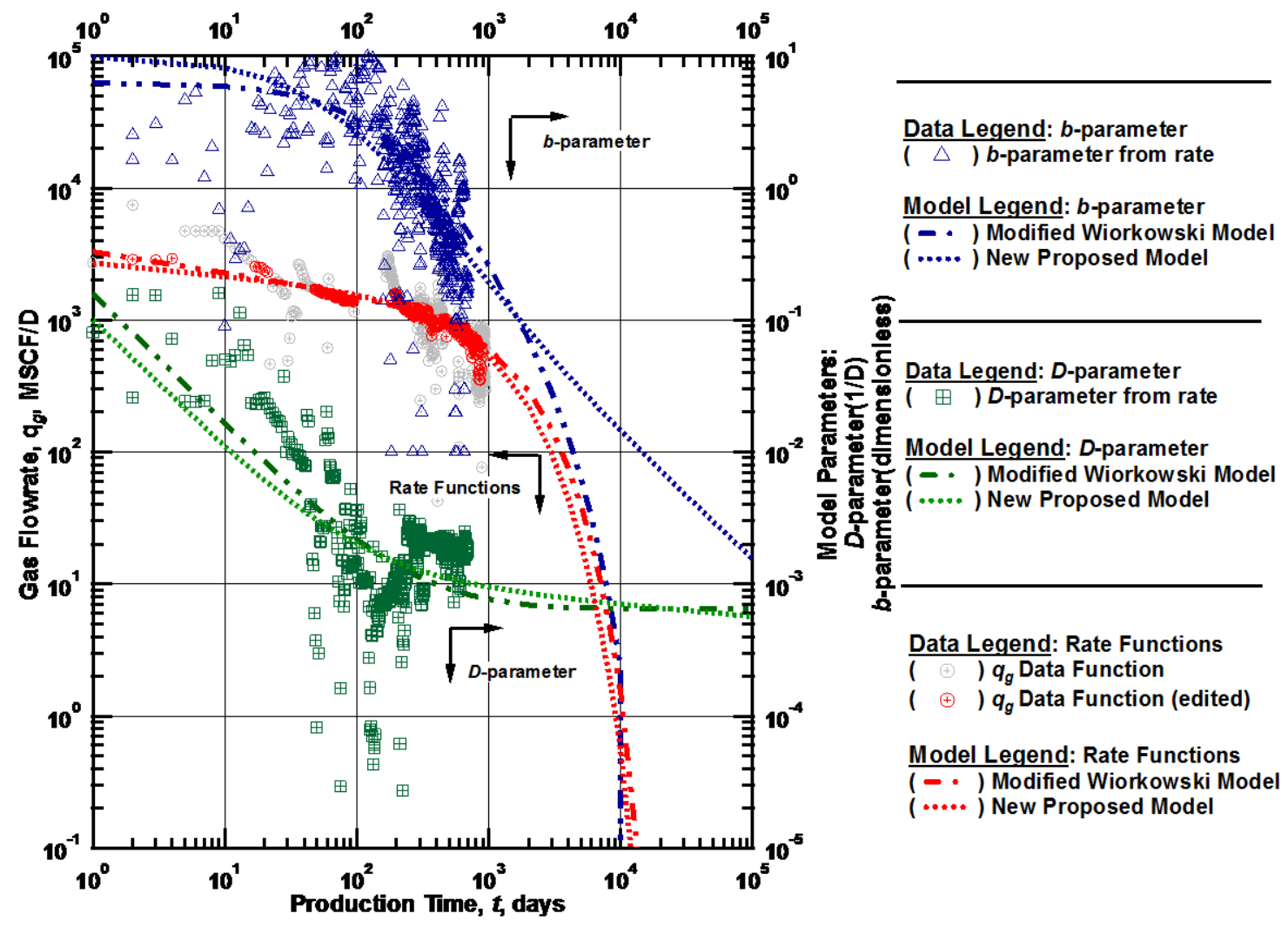

Figure E-22 - (Log-log Plot): $q D b$ plot - gas flow rate $\left(q_{g i}\right), D$ - and $b$-parameters versus production time and Modified Wiorkowski Model and Modified Ilk Model matches for Well 22. 
Modified Wiorkowksi and Proposed New Model qDb plot - Well 23

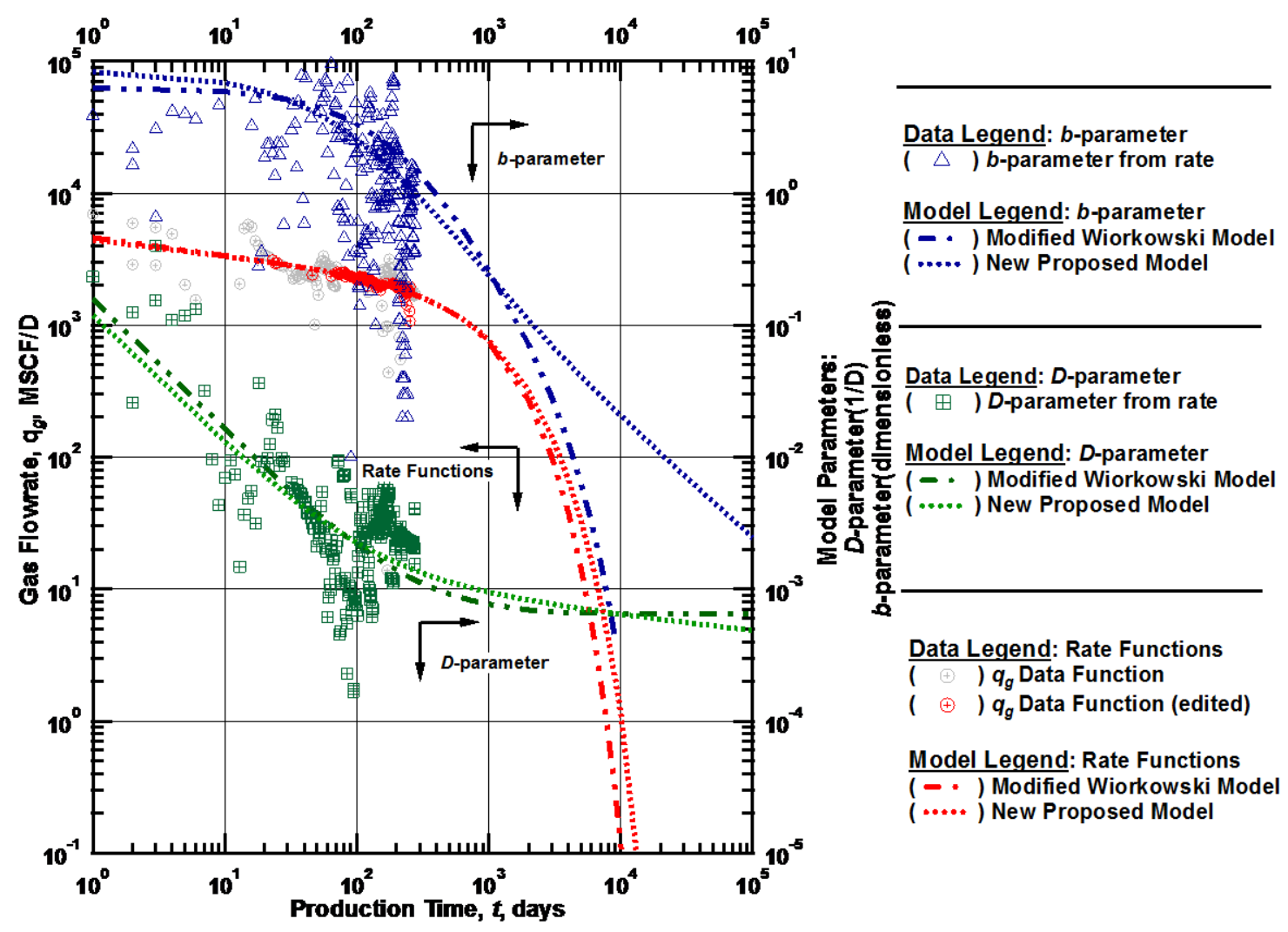

Figure E-23 - (Log-log Plot): $q D b$ plot - gas flow rate $\left(q_{g i}\right), D$ - and $b$-parameters versus production time and Modified Wiorkowski Model and Modified Ilk Model matches for Well 23. 
Modified Wiorkowksi and Proposed New Model qDb plot - Well 24

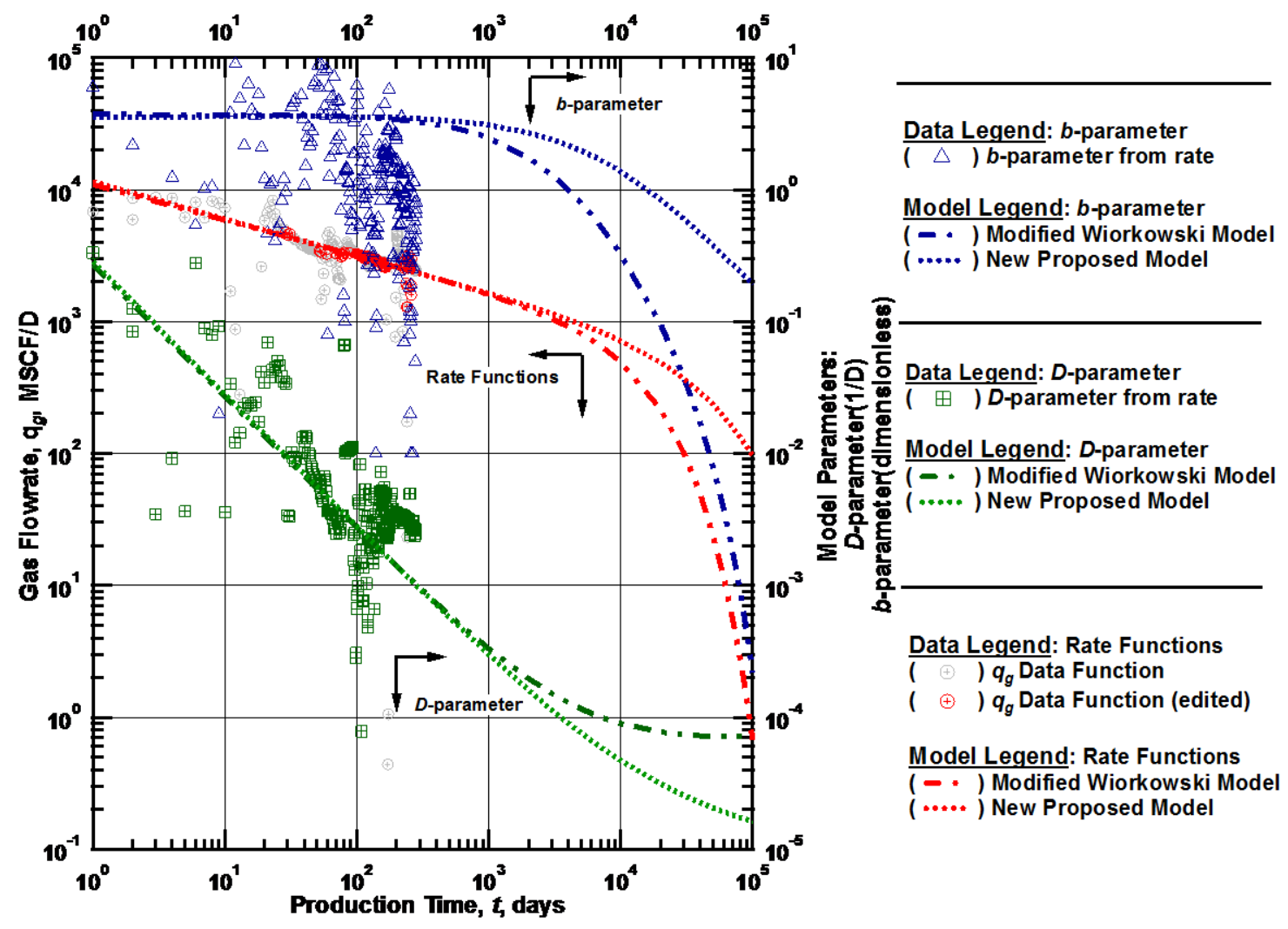

Figure E-24 - (Log-log Plot): $q D b$ plot - gas flow rate $\left(q_{g i}\right), D$ - and $b$-parameters versus production time and Modified Wiorkowski Model and Modified Ilk Model matches for Well 24. 
Modified Wiorkowksi and Proposed New Model qDb plot - Well 25

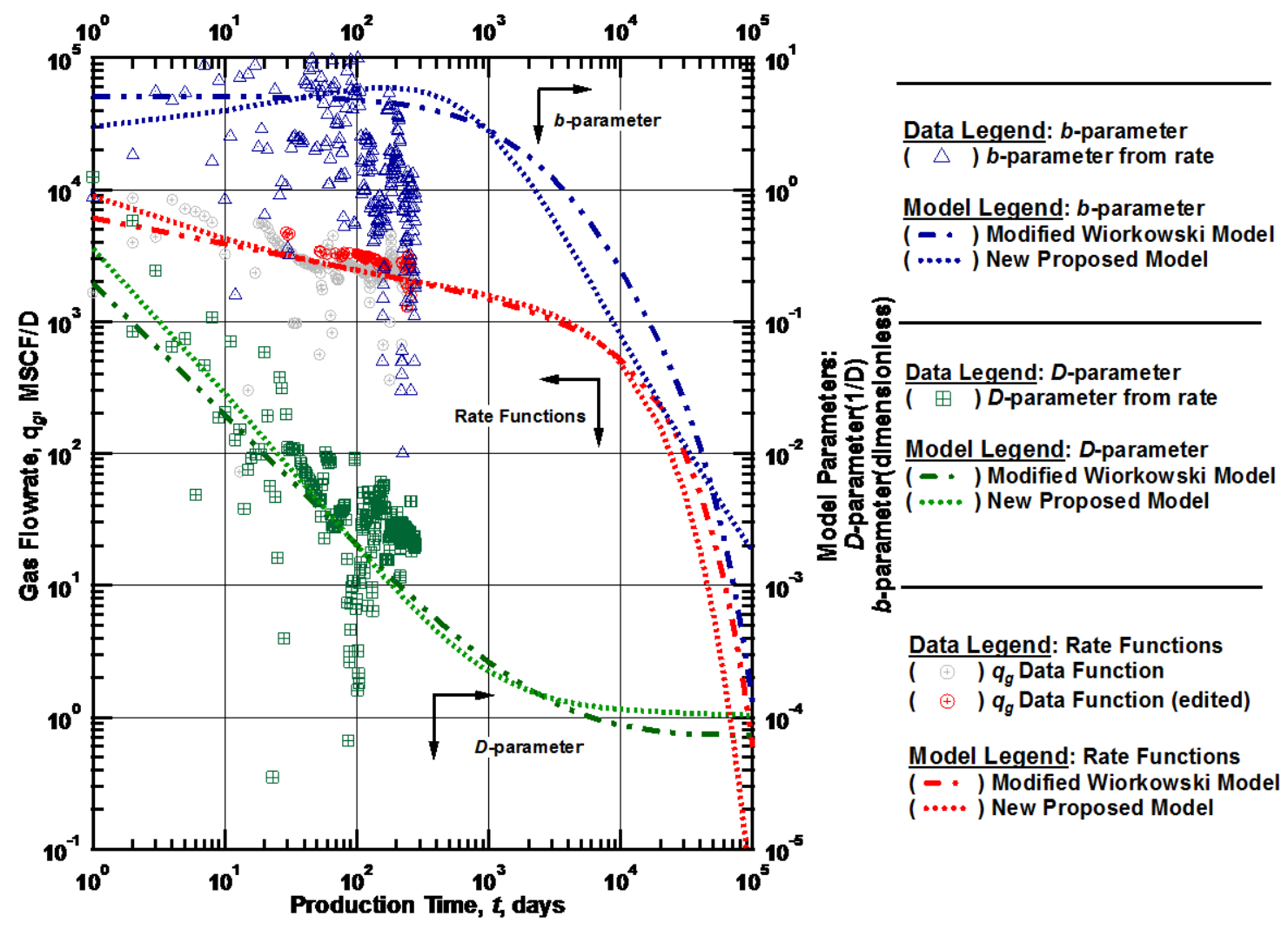

Figure E-25 - (Log-log Plot): $q D b$ plot - gas flow rate $\left(q_{g i}\right), D$ - and $b$-parameters versus production time and Modified Wiorkowski Model and Modified Ilk Model matches for Well 25. 
Modified Wiorkowksi and Proposed New Model qDb plot - Well 26

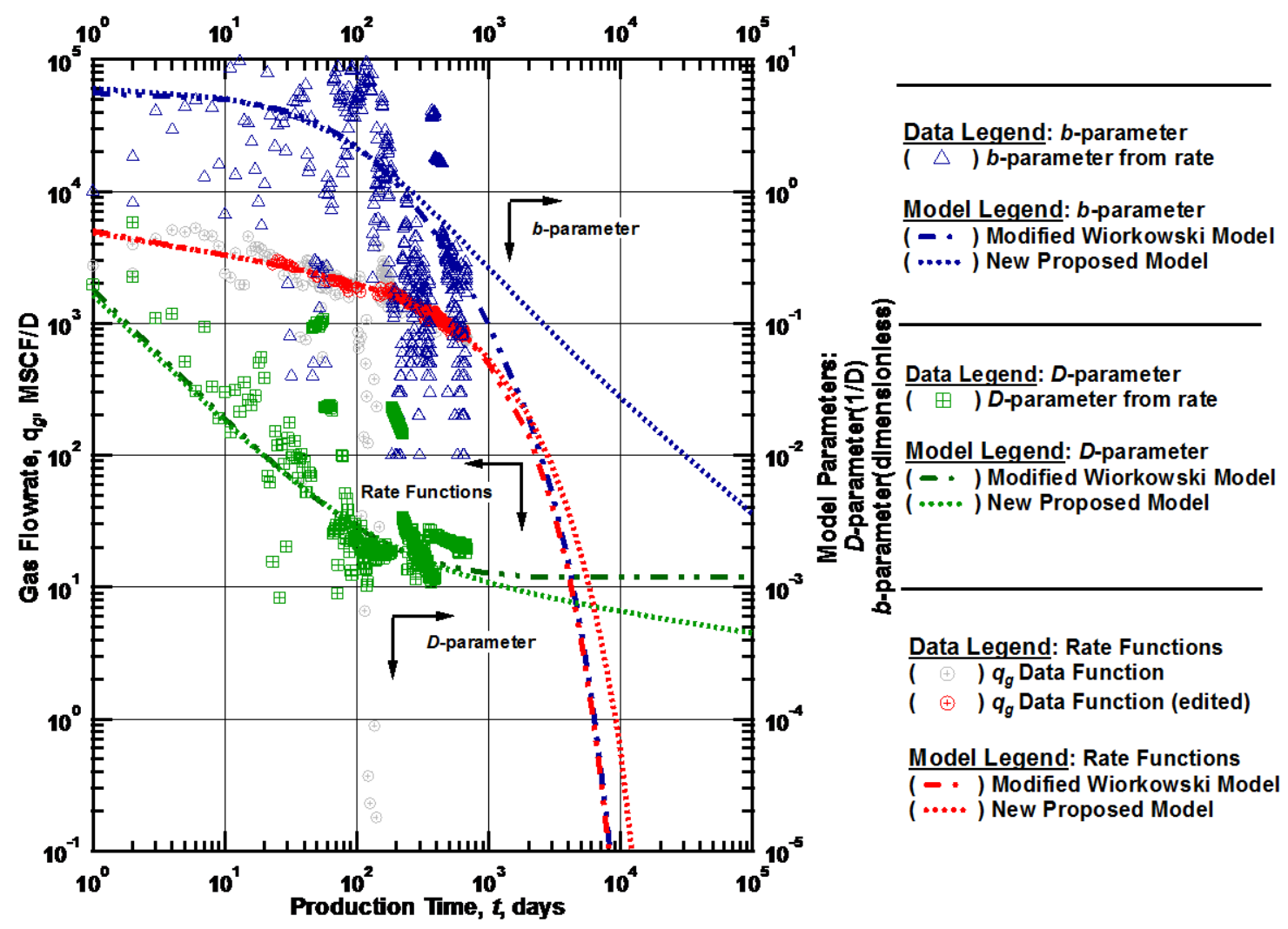

Figure E-26 - (Log-log Plot): $q D b$ plot - gas flow rate $\left(q_{g i}\right), D$ - and $b$-parameters versus production time and Modified Wiorkowski Model and Modified Ilk Model matches for Well 26. 
Modified Wiorkowksi and Proposed New Model qDb plot - Well 27

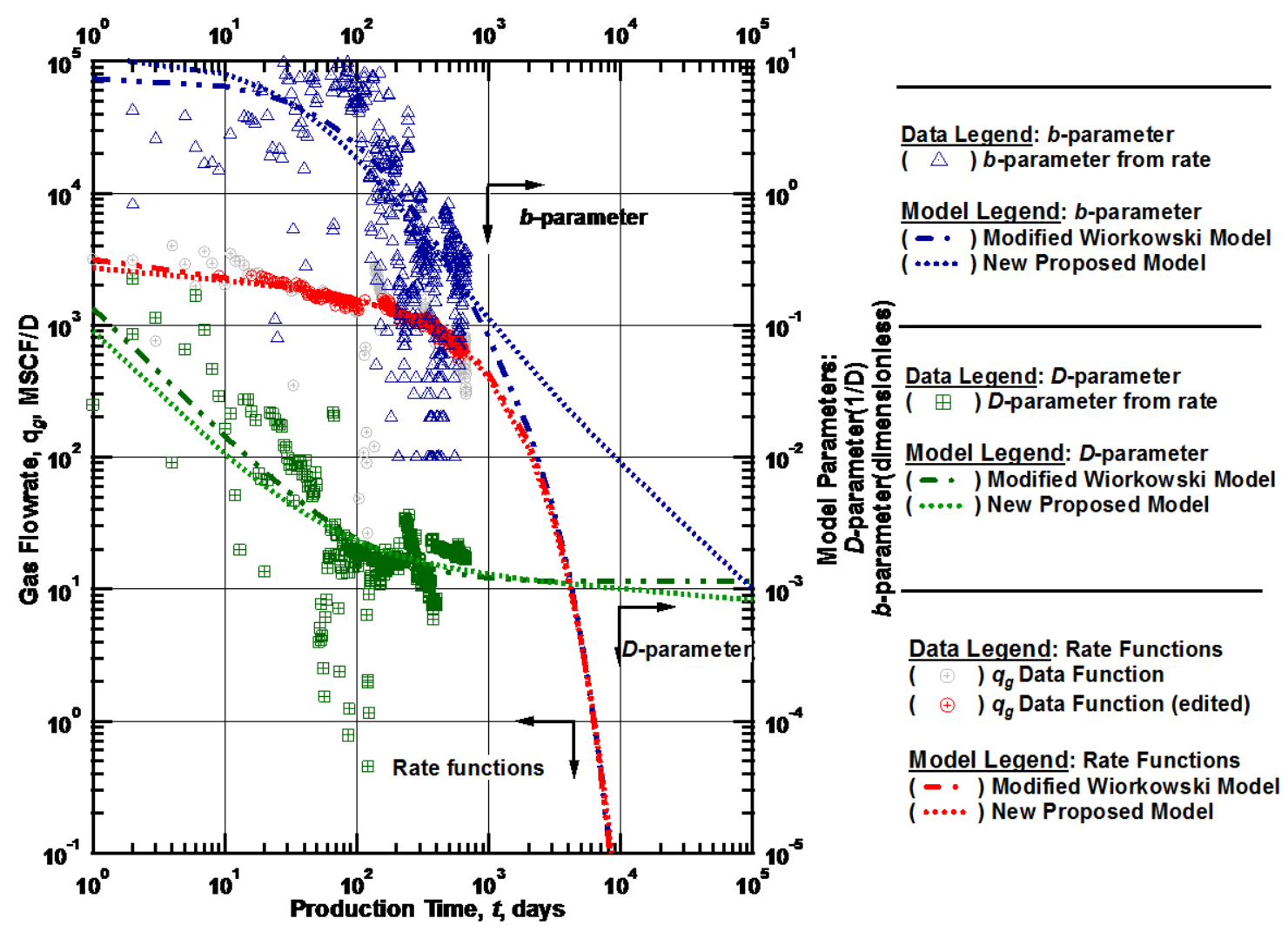

Figure E-27 - (Log-log Plot): $q D b$ plot — gas flow rate $\left(q_{g i}\right), D$ - and $b$-parameters versus production time and Modified Wiorkowski Model and Modified Ilk Model matches for Well 27. 
Modified Wiorkowksi and Proposed New Model qDb plot - Well 28

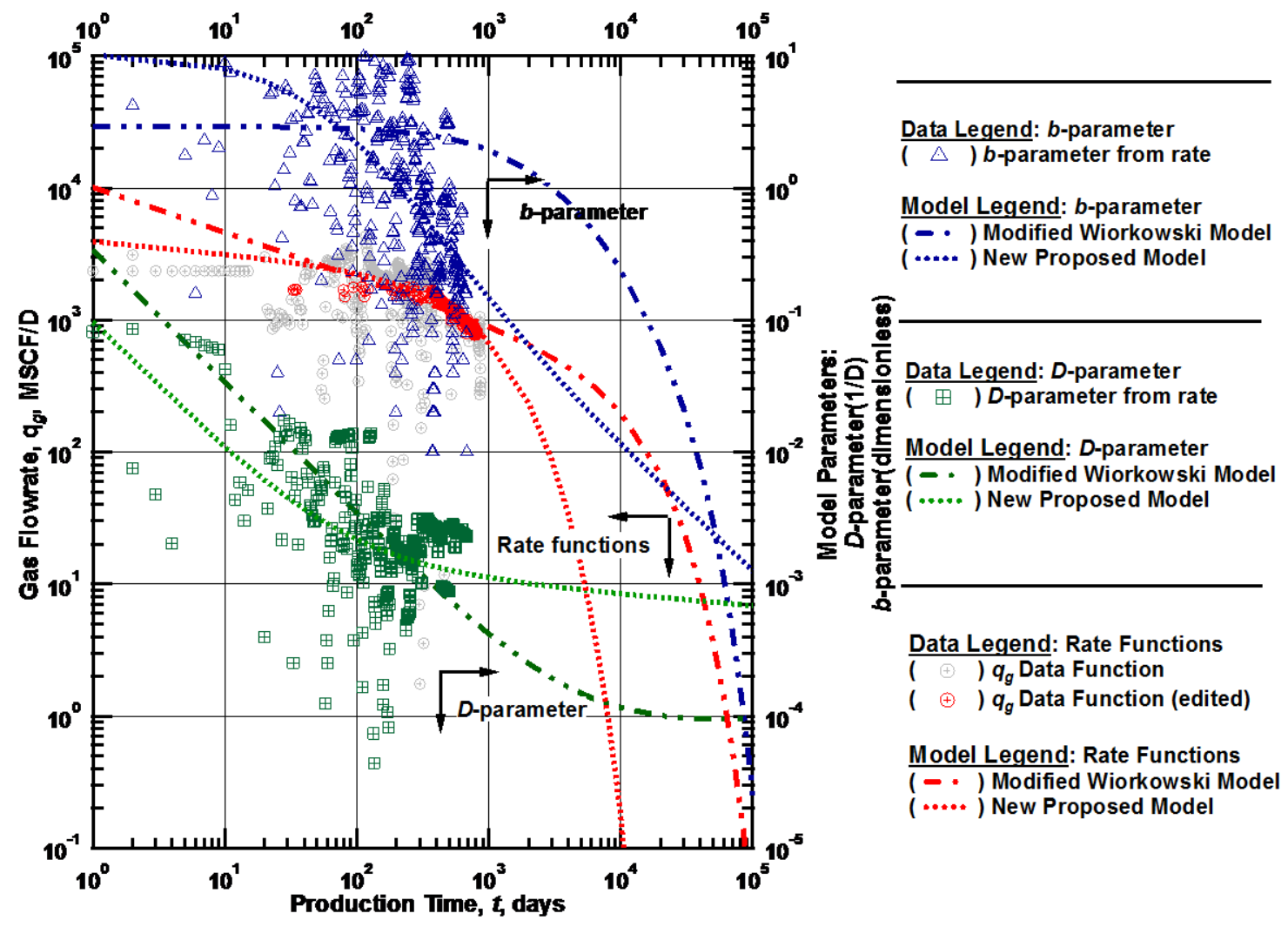

Figure E-28 - (Log-log Plot): $q D b$ plot - gas flow rate $\left(q_{g i}\right), D$ - and $b$-parameters versus production time and Modified Wiorkowski Model and Modified Ilk Model matches for Well 28. 
Modified Wiorkowksi and Proposed New Model qDb plot - Well 29

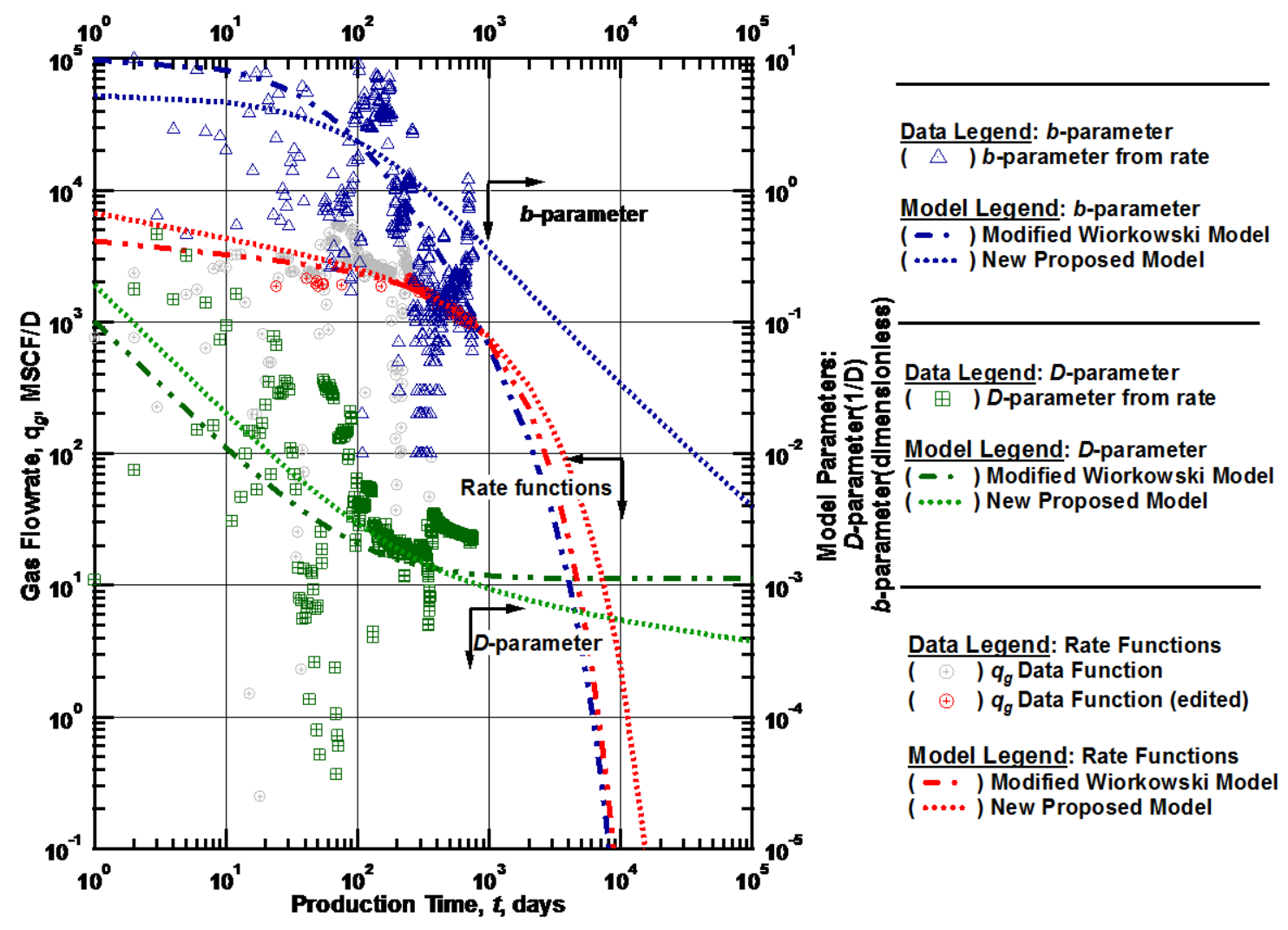

Figure E-29 - (Log-log Plot): $q D b$ plot - gas flow rate $\left(q_{g i}\right), D$ - and $b$-parameters versus production time and Modified Wiorkowski Model and Modified Ilk Model matches for Well 29. 
Modified Wiorkowksi and Proposed New Model qDb plot - Well 30

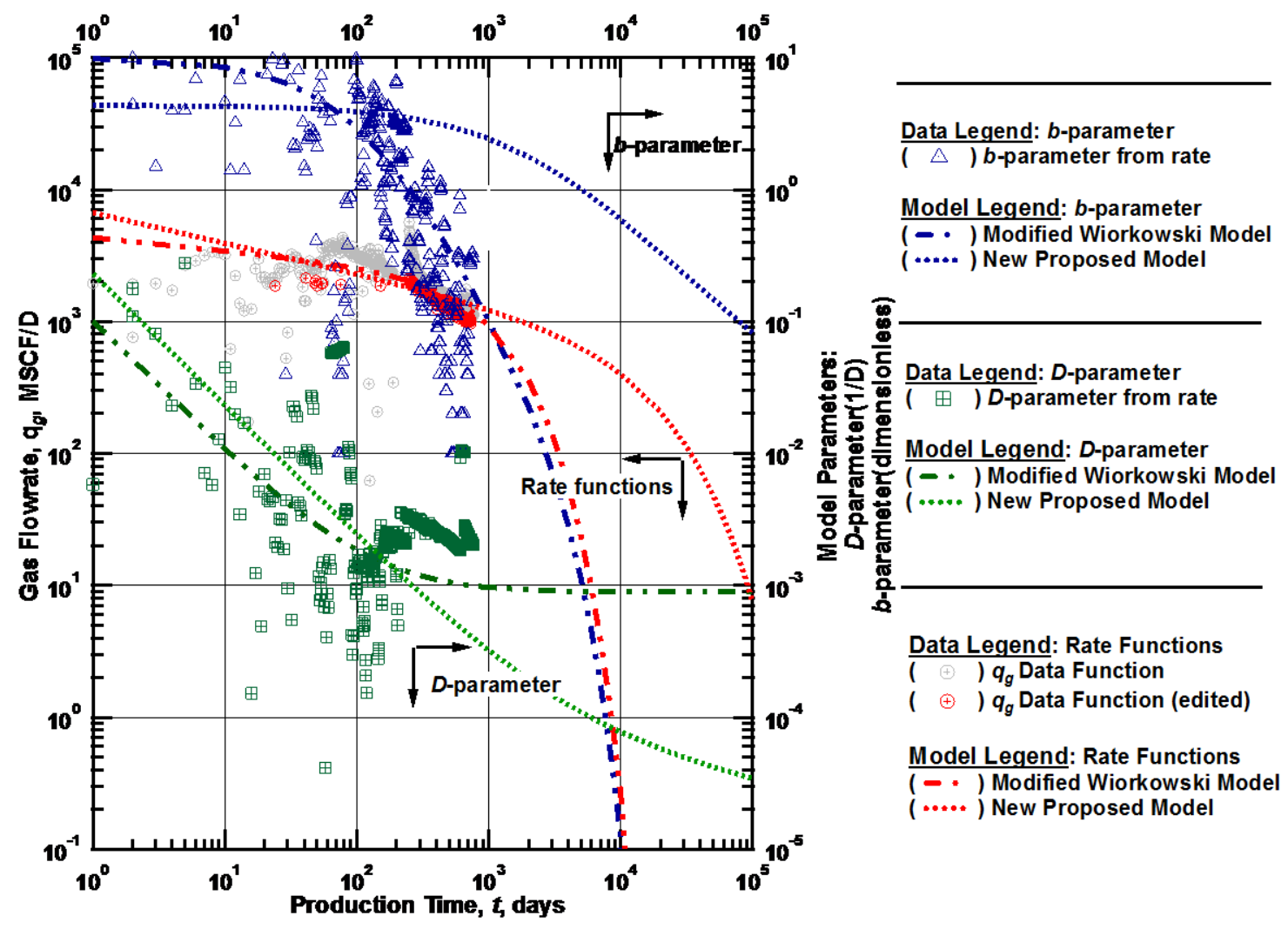

Figure E-30 - (Log-log Plot): $q D b$ plot — gas flow rate $\left(q_{g i}\right), D$ - and $b$-parameters versus production time and Modified Wiorkowski Model and Modified Ilk Model matches for Well 30. 
Modified Wiorkowksi and Proposed New Model qDb plot - Well 31

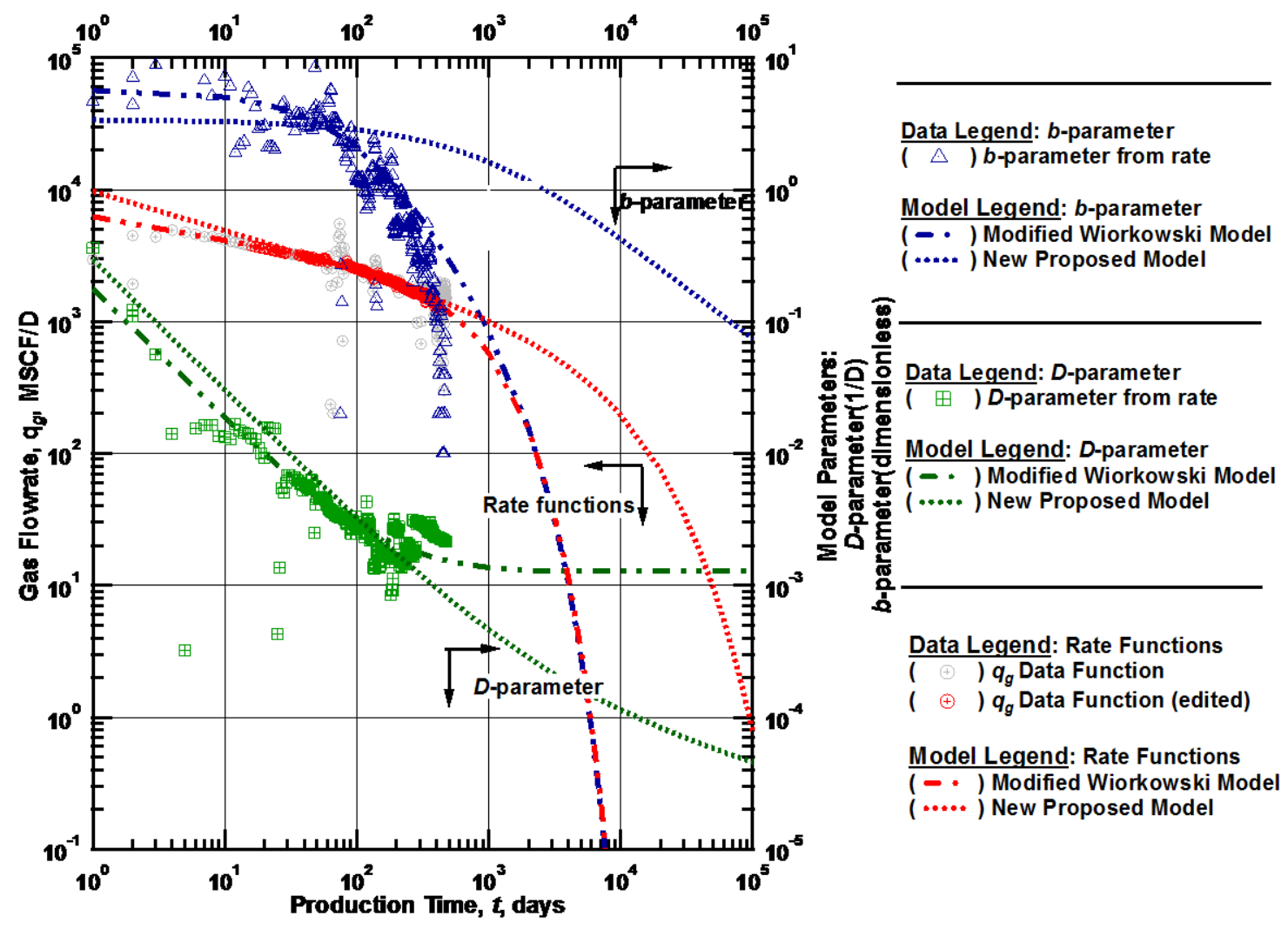

Figure E-31 - (Log-log Plot): $q D b$ plot - gas flow rate $\left(q_{g i}\right), D$ - and $b$-parameters versus production time and Modified Wiorkowski Model and Modified Ilk Model matches for Well 31. 
Modified Wiorkowksi and Proposed New Model qDb plot - Well 32

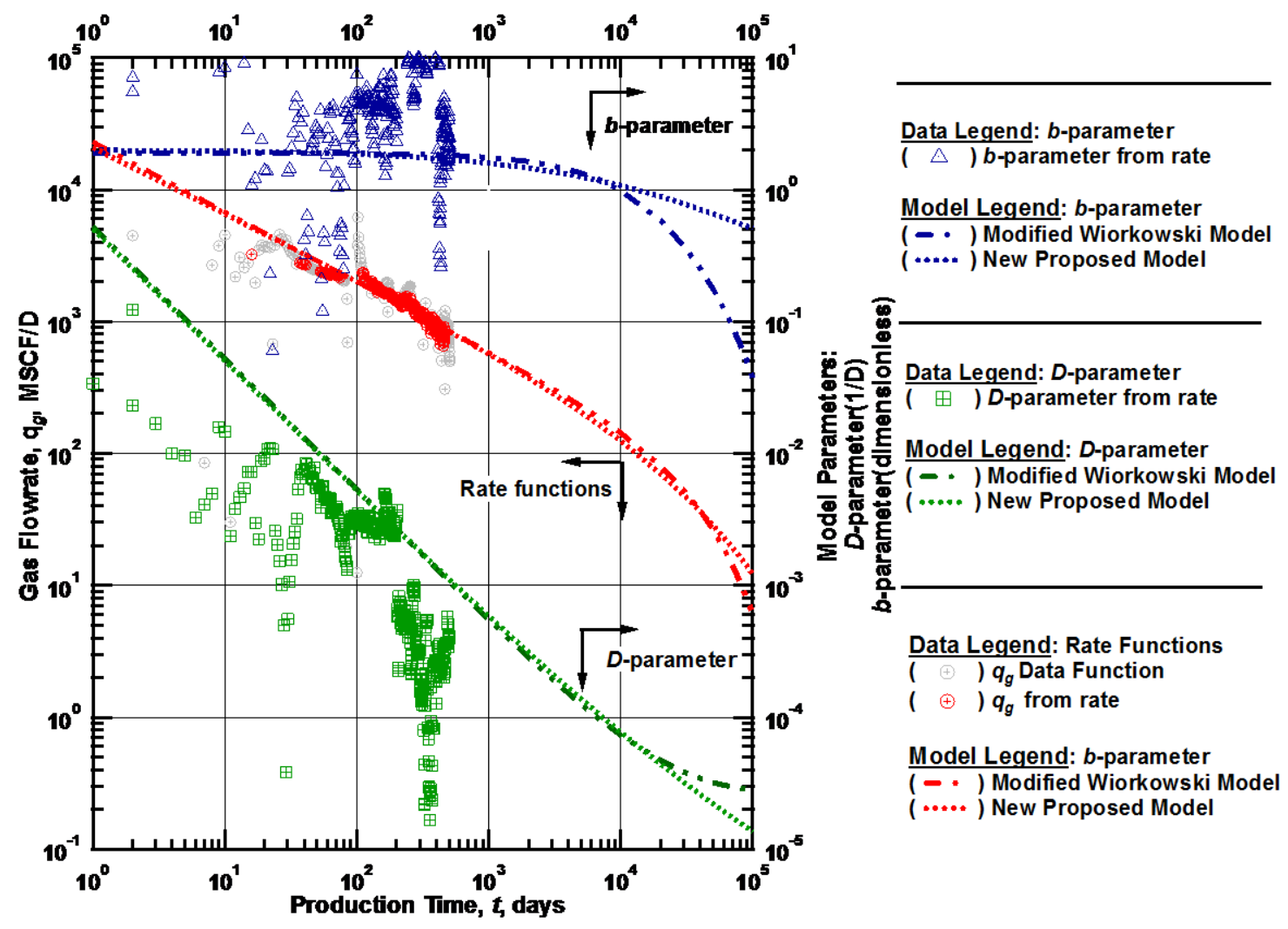

Figure E-32 - (Log-log Plot): $q D b$ plot — gas flow rate $\left(q_{g i}\right), D$ - and $b$-parameters versus production time and Modified Wiorkowski Model and Modified Ilk Model matches for Well 32. 
Modified Wiorkowksi and Proposed New Model qDb plot - Well 33

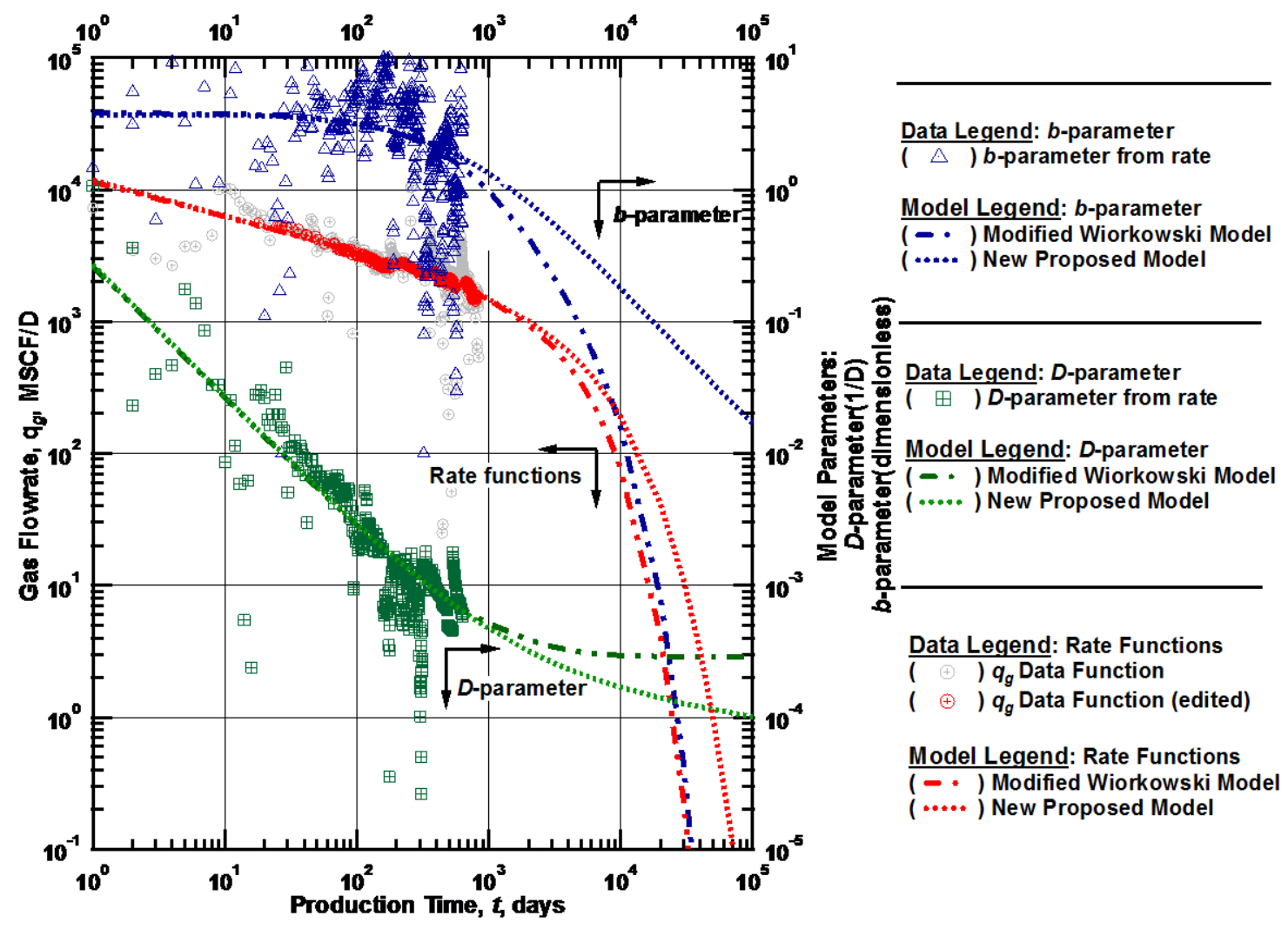

Figure E-33 - (Log-log Plot): $q D b$ plot — gas flow rate $\left(q_{g i}\right), D$ - and $b$-parameters versus production time and Modified Wiorkowski Model and Modified Ilk Model matches for Well 33. 
Modified Wiorkowksi and Proposed New Model qDb plot - Well 34

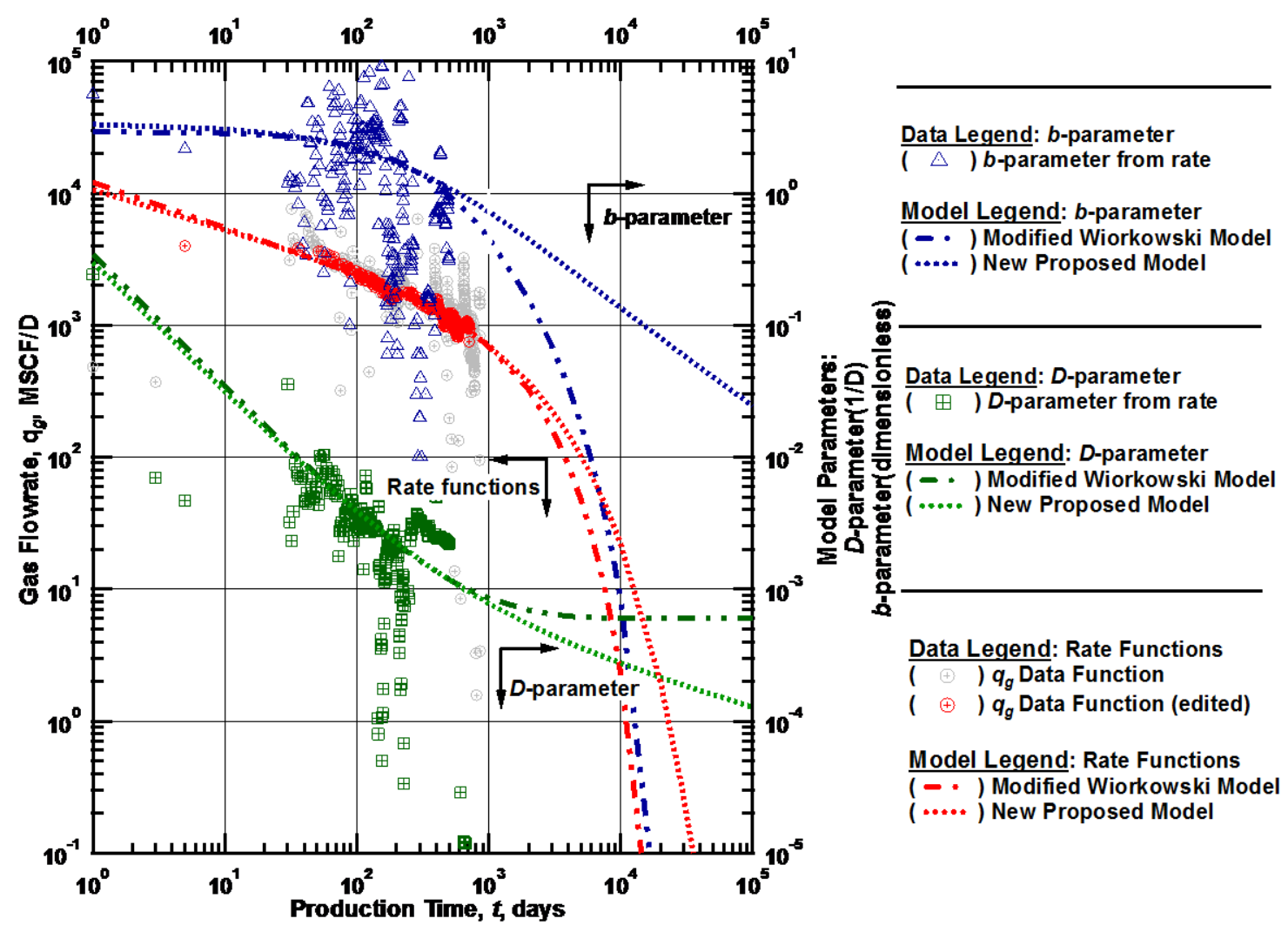

Figure E-34 - (Log-log Plot): $q D b$ plot — gas flow rate $\left(q_{g i}\right), D$ - and $b$-parameters versus production time and Modified Wiorkowski Model and Modified Ilk Model matches for Well 34. 
Modified Wiorkowksi and Proposed New Model qDb plot - Well 35

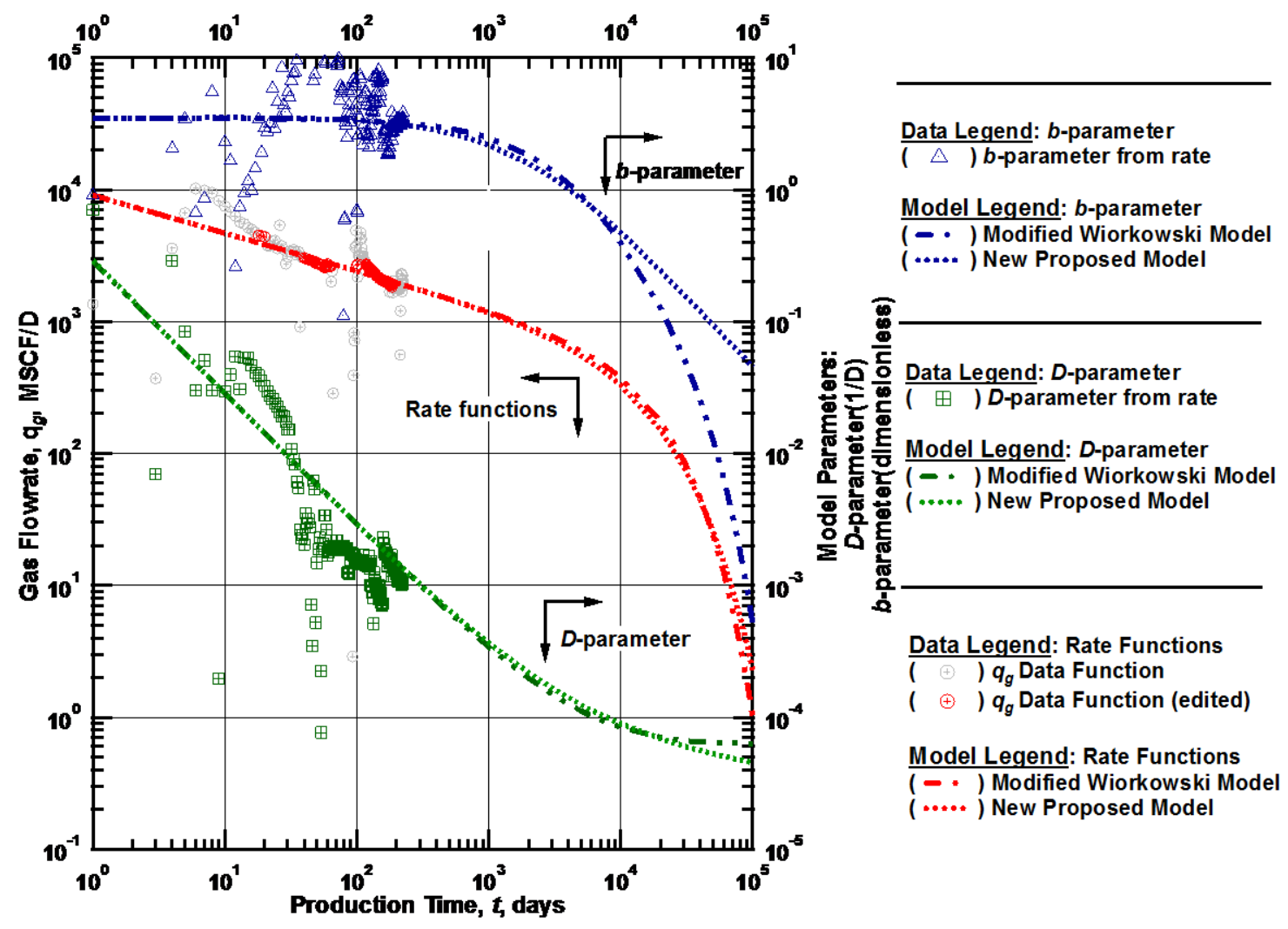

Figure E-35 - (Log-log Plot): $q D b$ plot — gas flow rate $\left(q_{g i}\right), D$ - and $b$-parameters versus production time and Modified Wiorkowski Model and Modified Ilk Model matches for Well 35. 
Modified Wiorkowksi and Proposed New Model qDb plot - Well 36

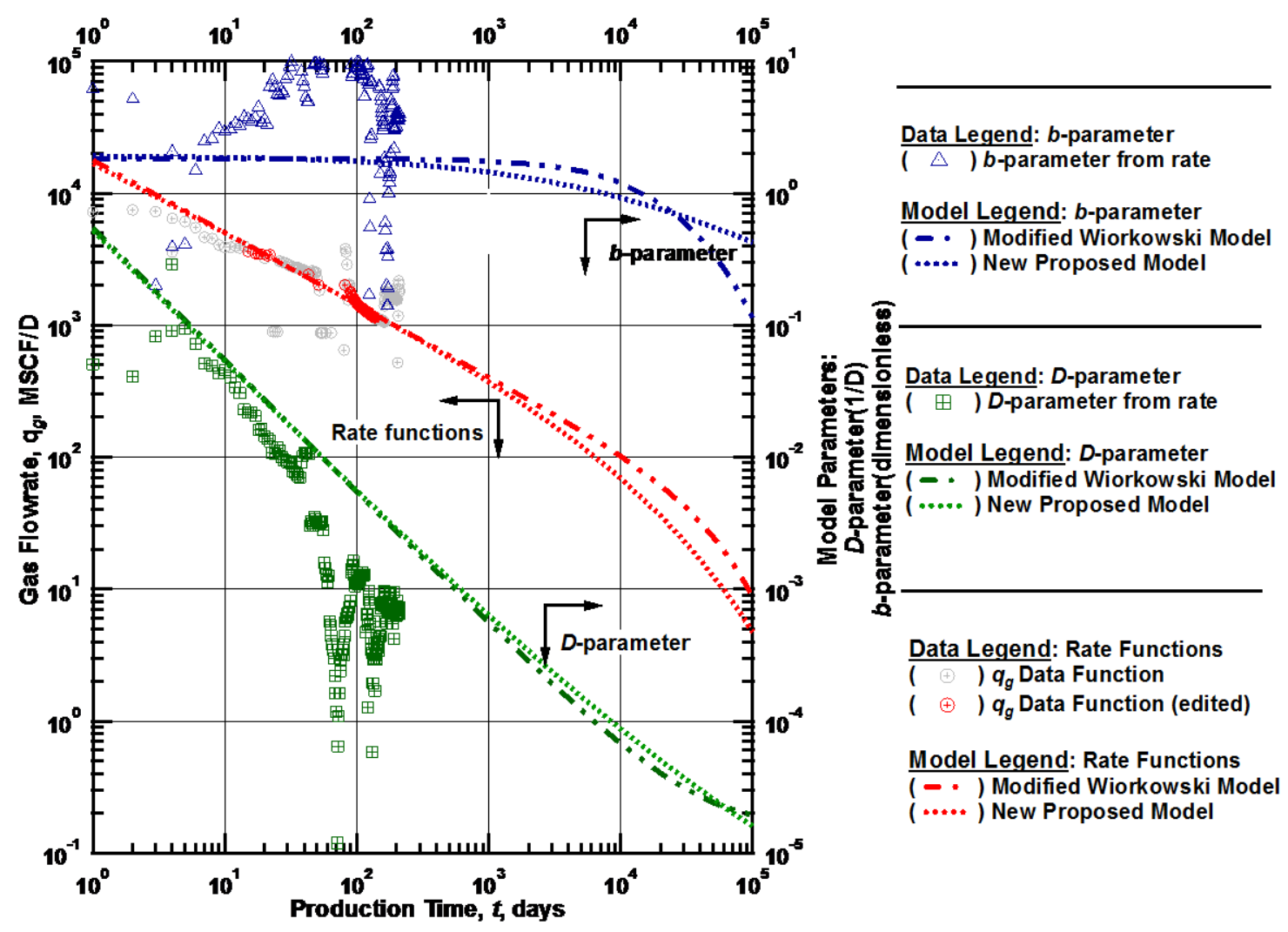

Figure E-36 - (Log-log Plot): $q D b$ plot — gas flow rate $\left(q_{g i}\right), D$ - and $b$-parameters versus production time and Modified Wiorkowski Model and Modified Ilk Model matches for Well 36. 
Modified Wiorkowksi and Proposed New Model qDb plot - Well 37

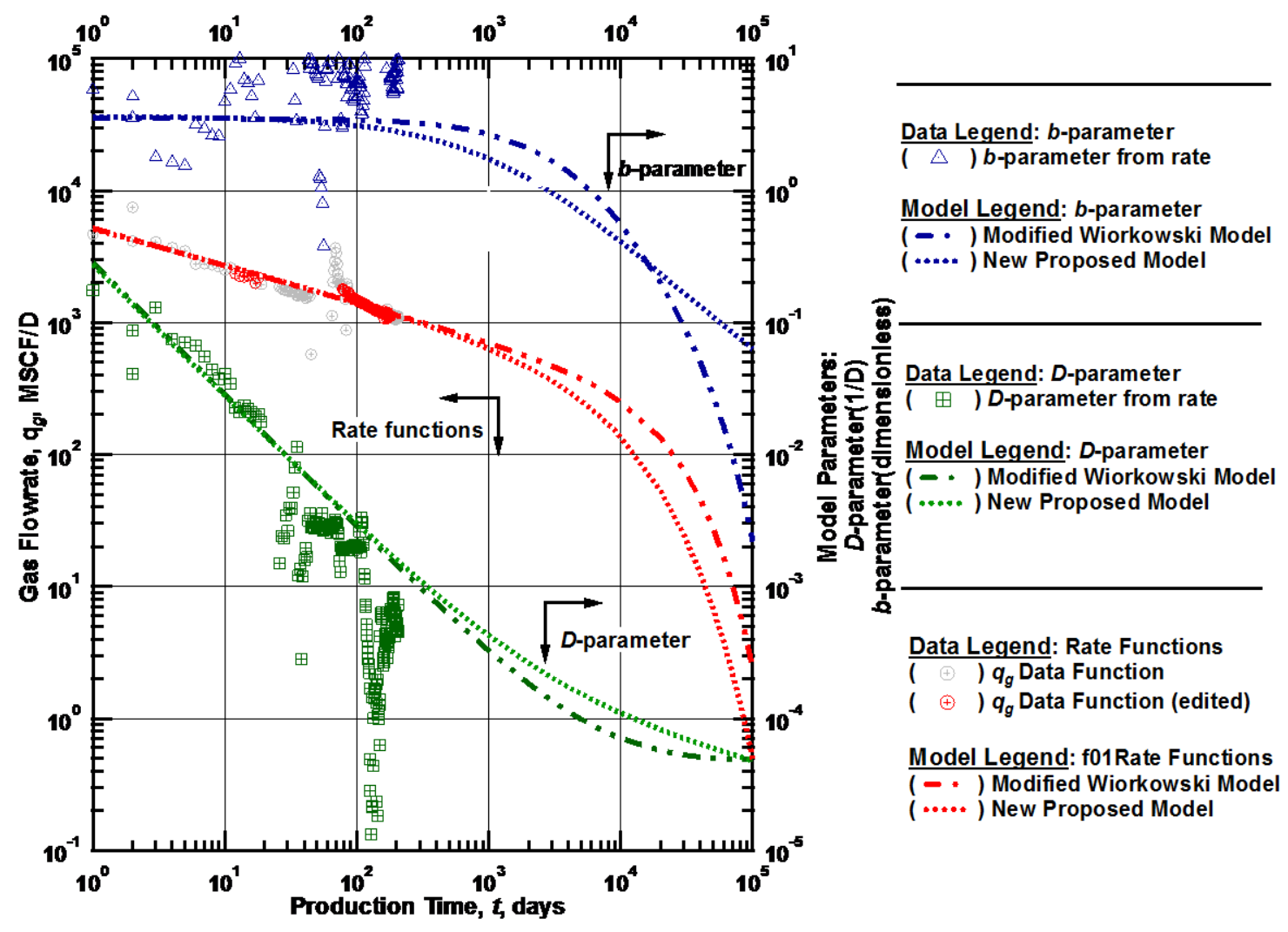

Figure E-37 - (Log-log Plot): $q D b$ plot — gas flow rate $\left(q_{g i}\right), D$ - and $b$-parameters versus production time and Modified Wiorkowski Model and Modified Ilk Model matches for Well 37. 
Modified Wiorkowksi and Proposed New Model qDb plot - Well 38

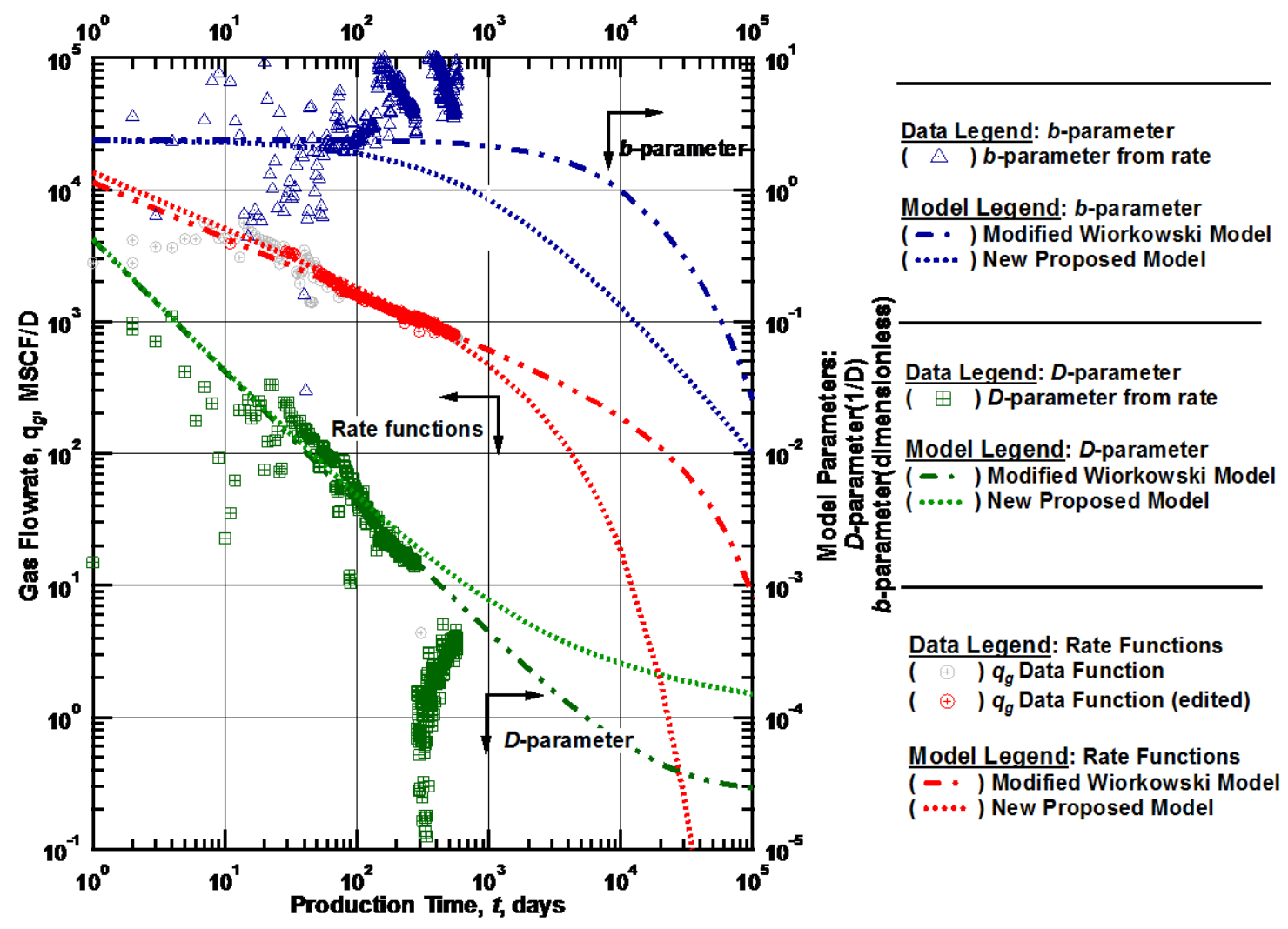

Figure E-38 - (Log-log Plot): $q D b$ plot - gas flow rate $\left(q_{g i}\right), D$ - and $b$-parameters versus production time and Modified Wiorkowski Model and Modified Ilk Model matches for Well 38. 
Modified Wiorkowksi and Proposed New Model qDb plot - Well 39

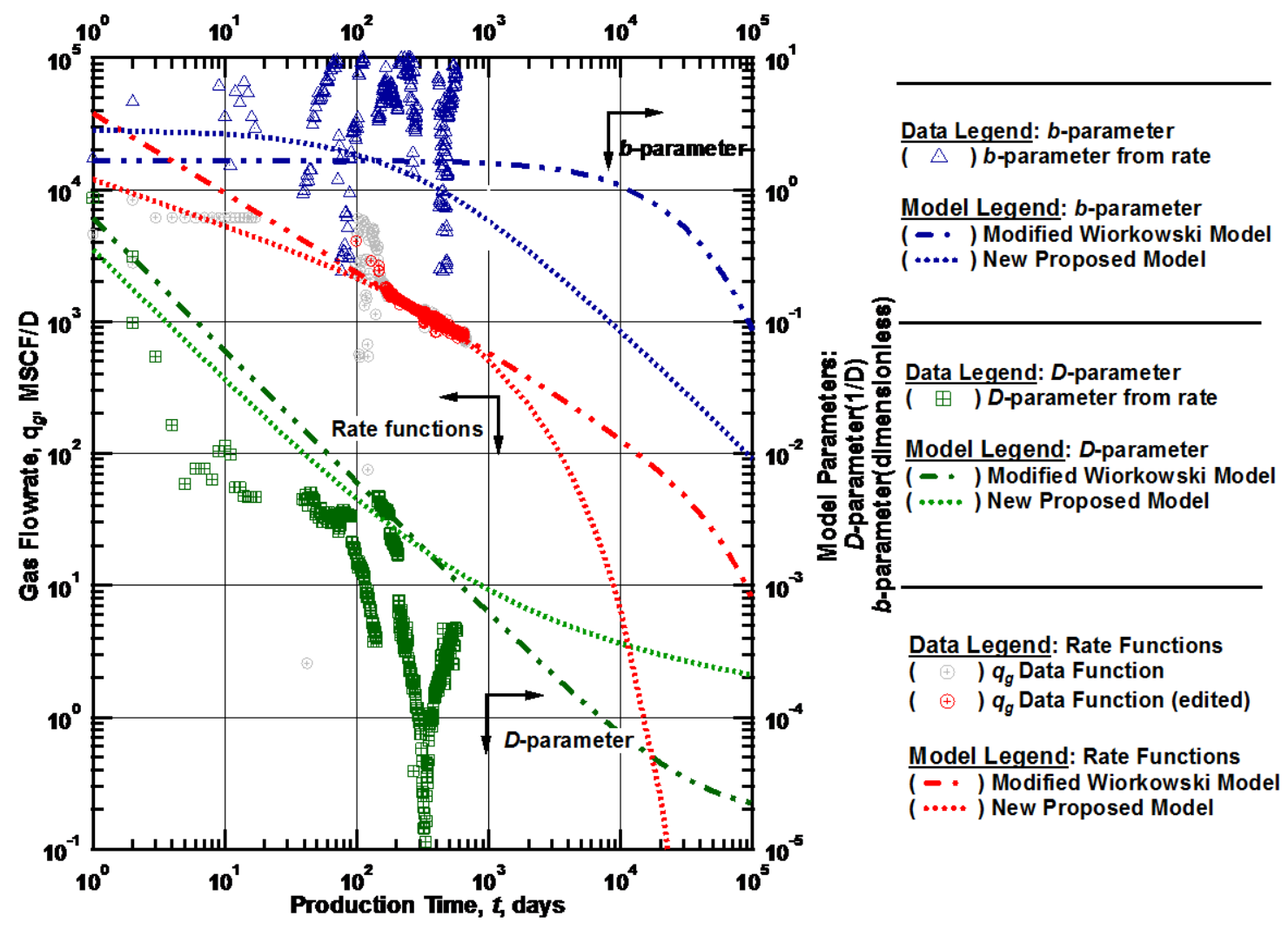

Figure E-39 - (Log-log Plot): $q D b$ plot - gas flow rate $\left(q_{g i}\right), D$ - and $b$-parameters versus production time and Modified Wiorkowski Model and Modified Ilk Model matches for Well 39. 
Modified Wiorkowksi and Proposed New Model qDb plot - Well 40

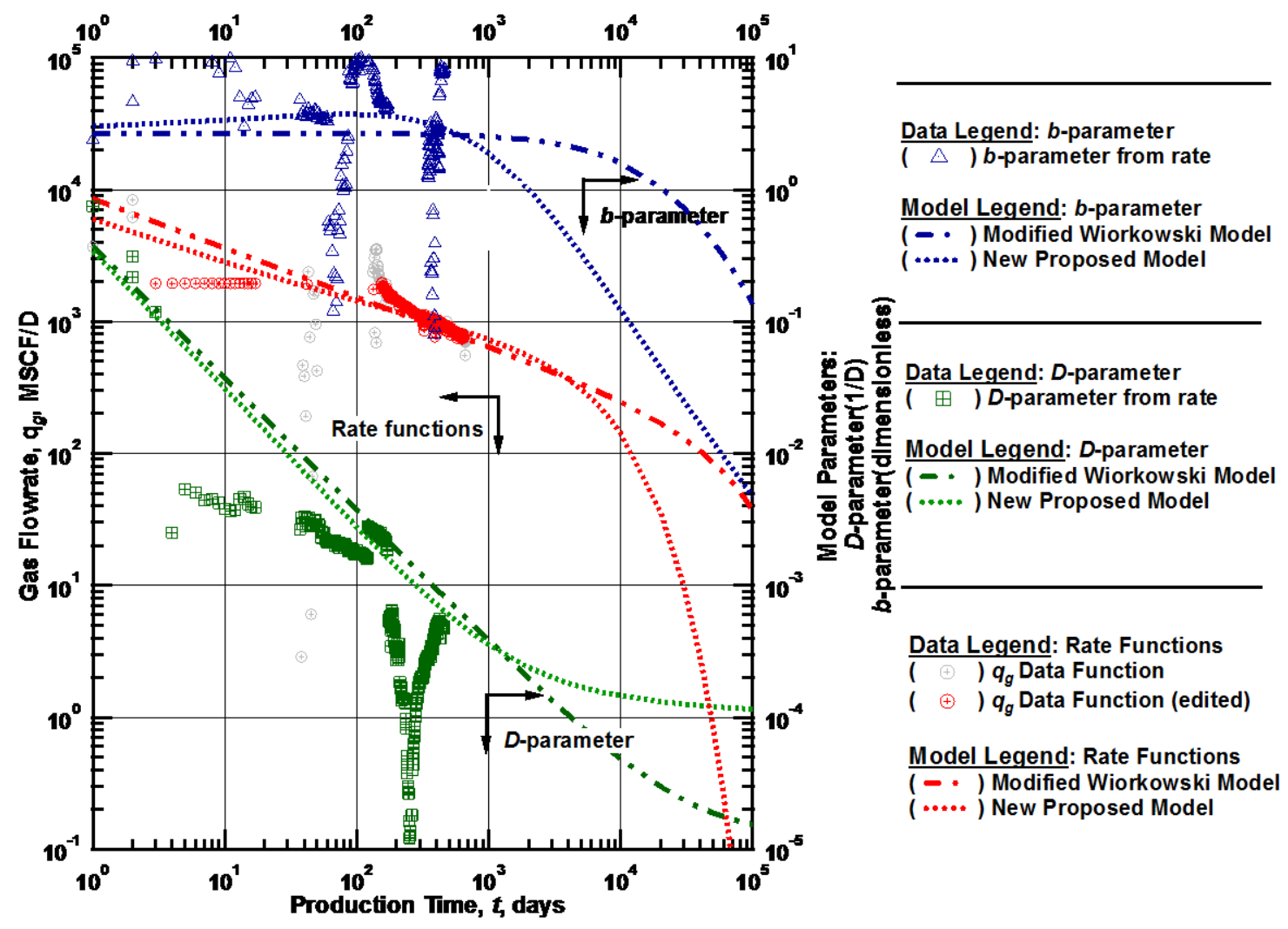

Figure E-40 - (Log-log Plot): $q D b$ plot - gas flow rate $\left(q_{g i}\right), D$ - and $b$-parameters versus production time and Modified Wiorkowski Model and Modified Ilk Model matches for Well 40. 
Modified Wiorkowksi and Proposed New Model qDb plot - Well 41

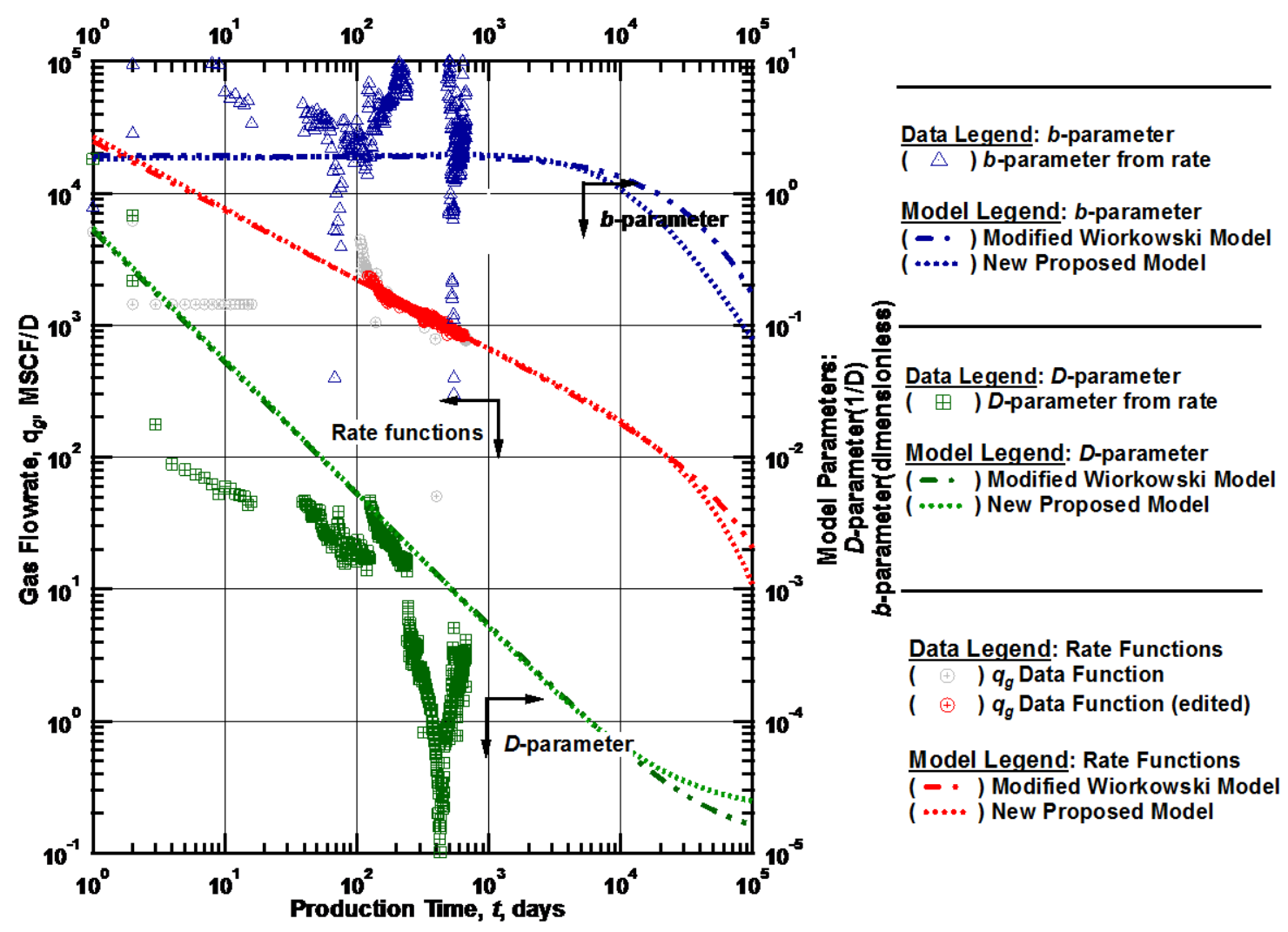

Figure E-41 - (Log-log Plot): $q D b$ plot — gas flow rate $\left(q_{g i}\right), D$ - and $b$-parameters versus production time and Modified Wiorkowski Model and Modified Ilk Model matches for Well 41. 
Modified Wiorkowksi and Proposed New Model qDb plot - Well 42

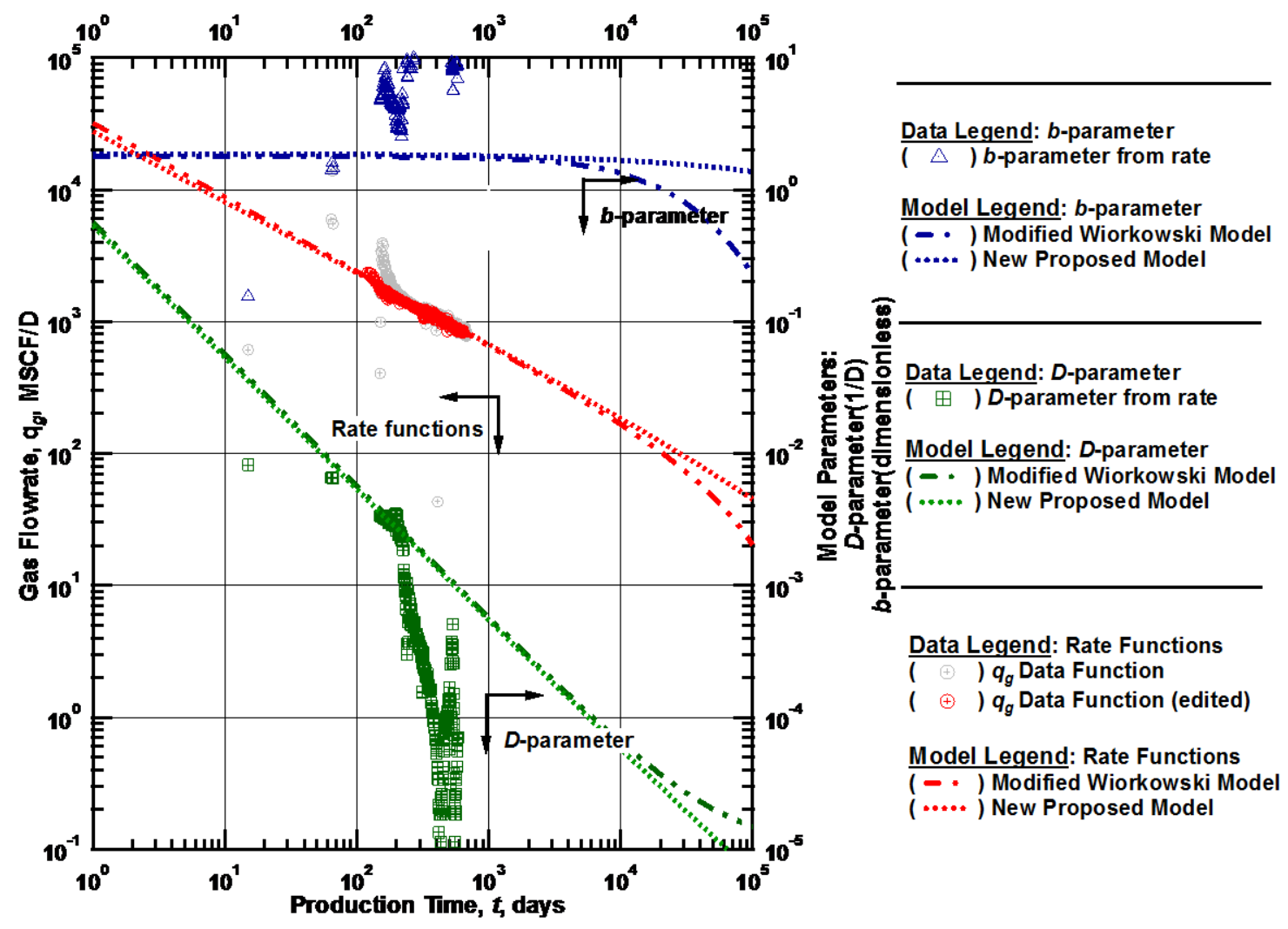

Figure E-42 - (Log-log Plot): $q D b$ plot — gas flow rate $\left(q_{g i}\right), D$ - and $b$-parameters versus production time and Modified Wiorkowski Model and Modified Ilk Model matches for Well 42. 
Modified Wiorkowksi and Proposed New Model qDb plot - Well 43

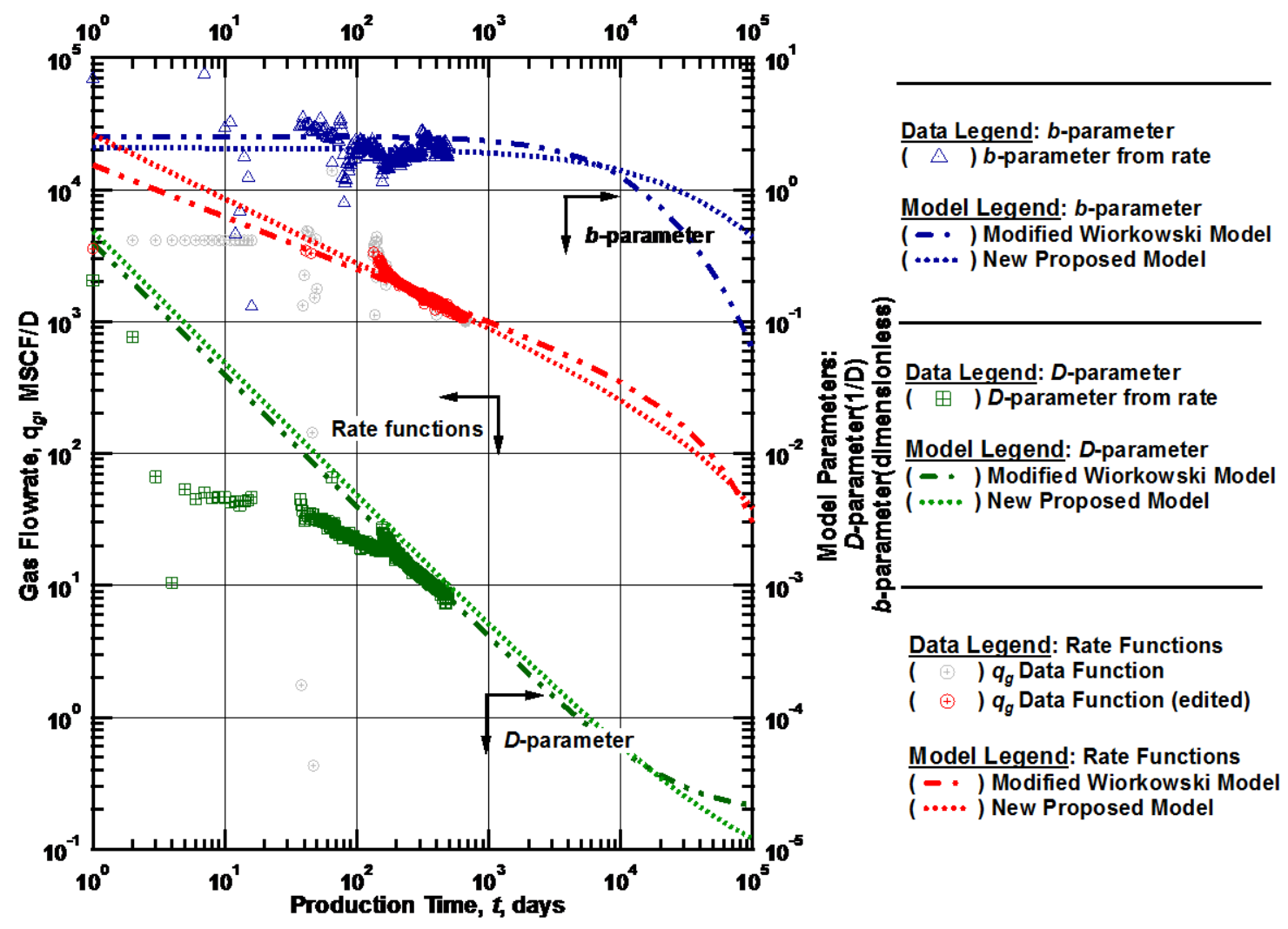

Figure E-43 - (Log-log Plot): $q D b$ plot - gas flow rate $\left(q_{g i}\right), D$ - and $b$-parameters versus production time and Modified Wiorkowski Model and Modified Ilk Model matches for Well 43. 
Modified Wiorkowksi and Proposed New Model qDb plot - Well 44

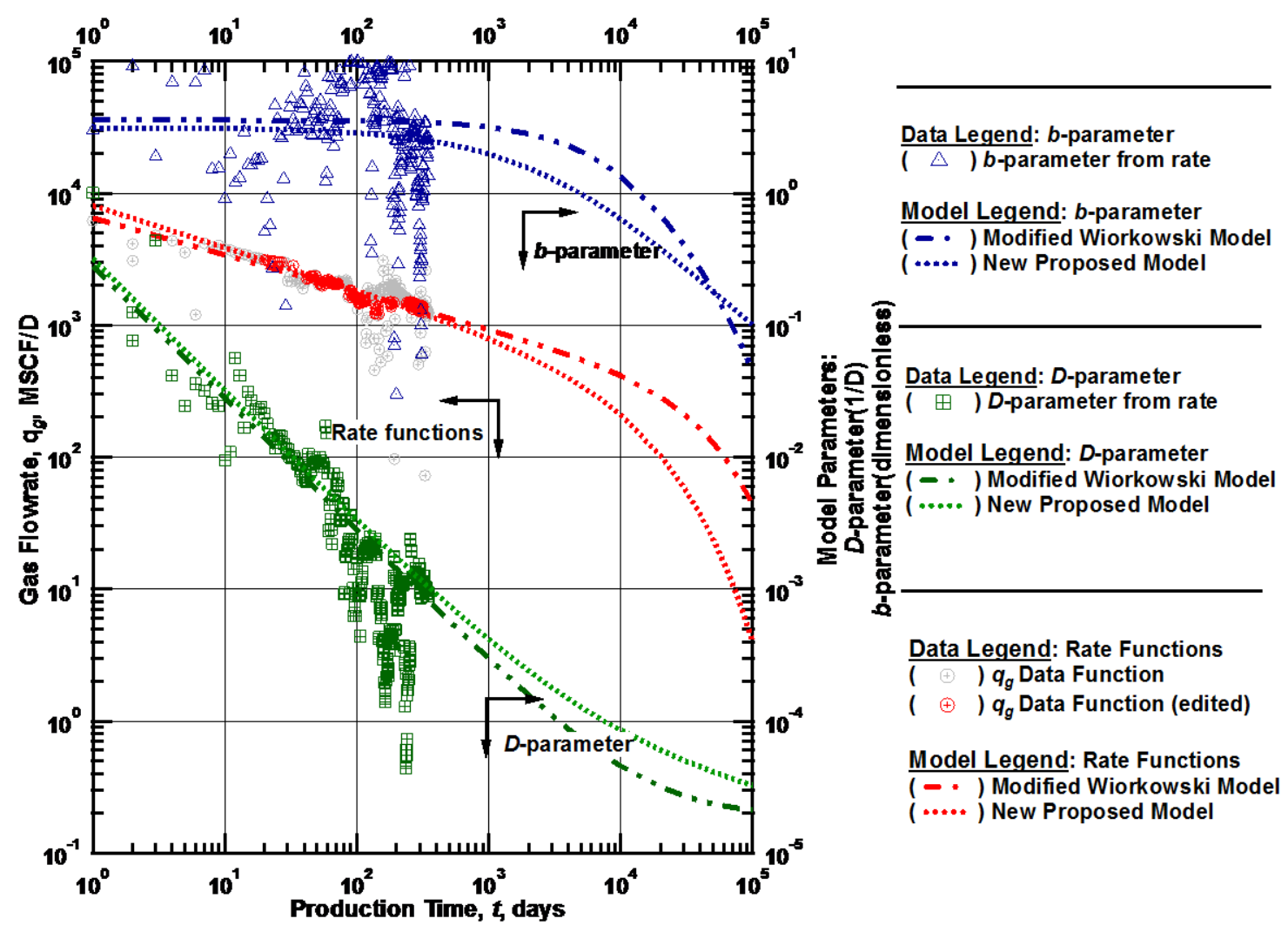

Figure E-44 - (Log-log Plot): $q D b$ plot — gas flow rate $\left(q_{g i}\right), D$ - and $b$-parameters versus production time and Modified Wiorkowski Model and Modified Ilk Model matches for Well 44. 
Modified Wiorkowksi and Proposed New Model qDb plot - Well 45

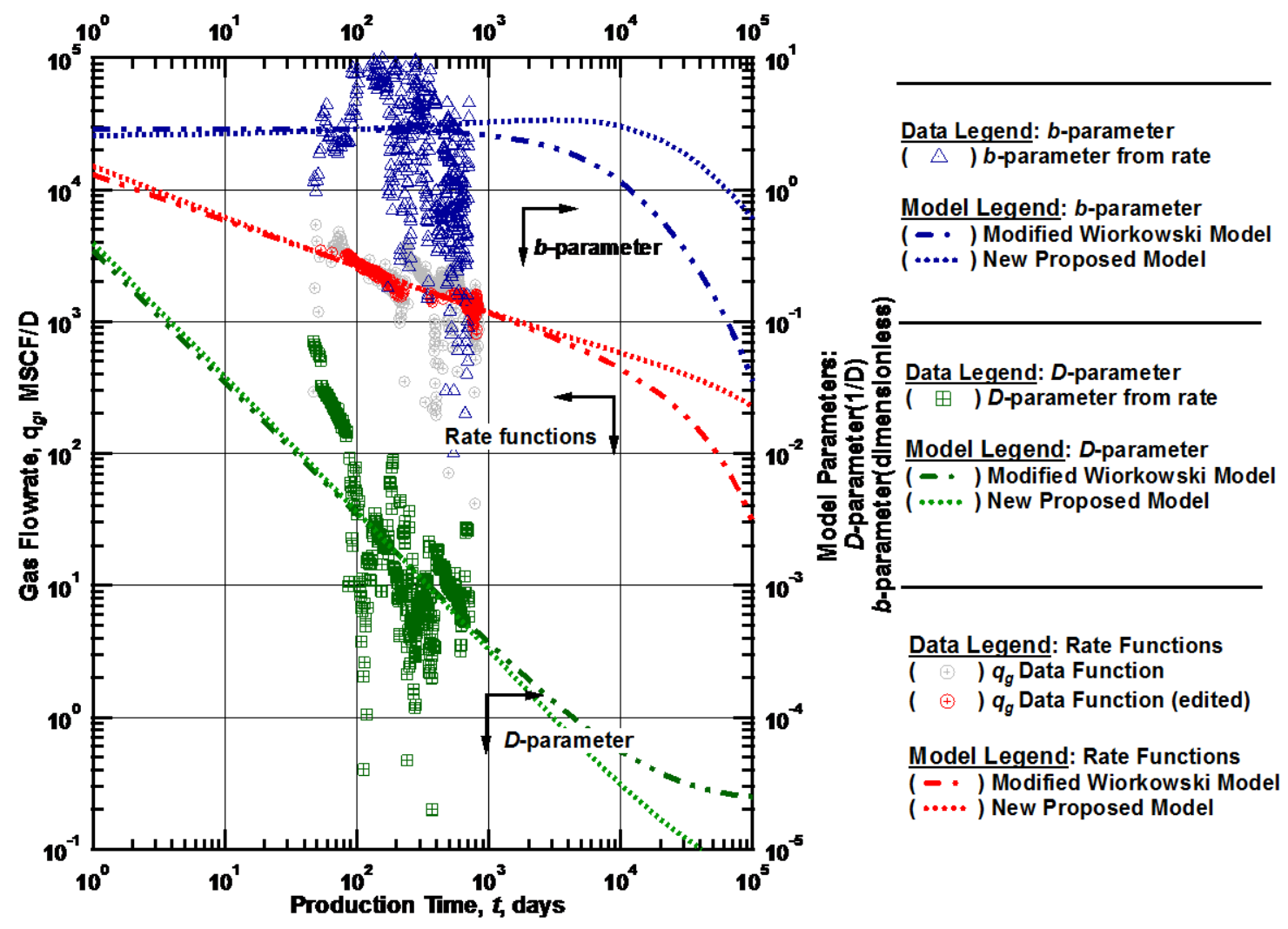

Figure E-45 - (Log-log Plot): $q D b$ plot - gas flow rate $\left(q_{g i}\right), D$ - and $b$-parameters versus production time and Modified Wiorkowski Model and Modified Ilk Model matches for Well 45. 
Modified Wiorkowksi and Proposed New Model qDb plot - Well 46

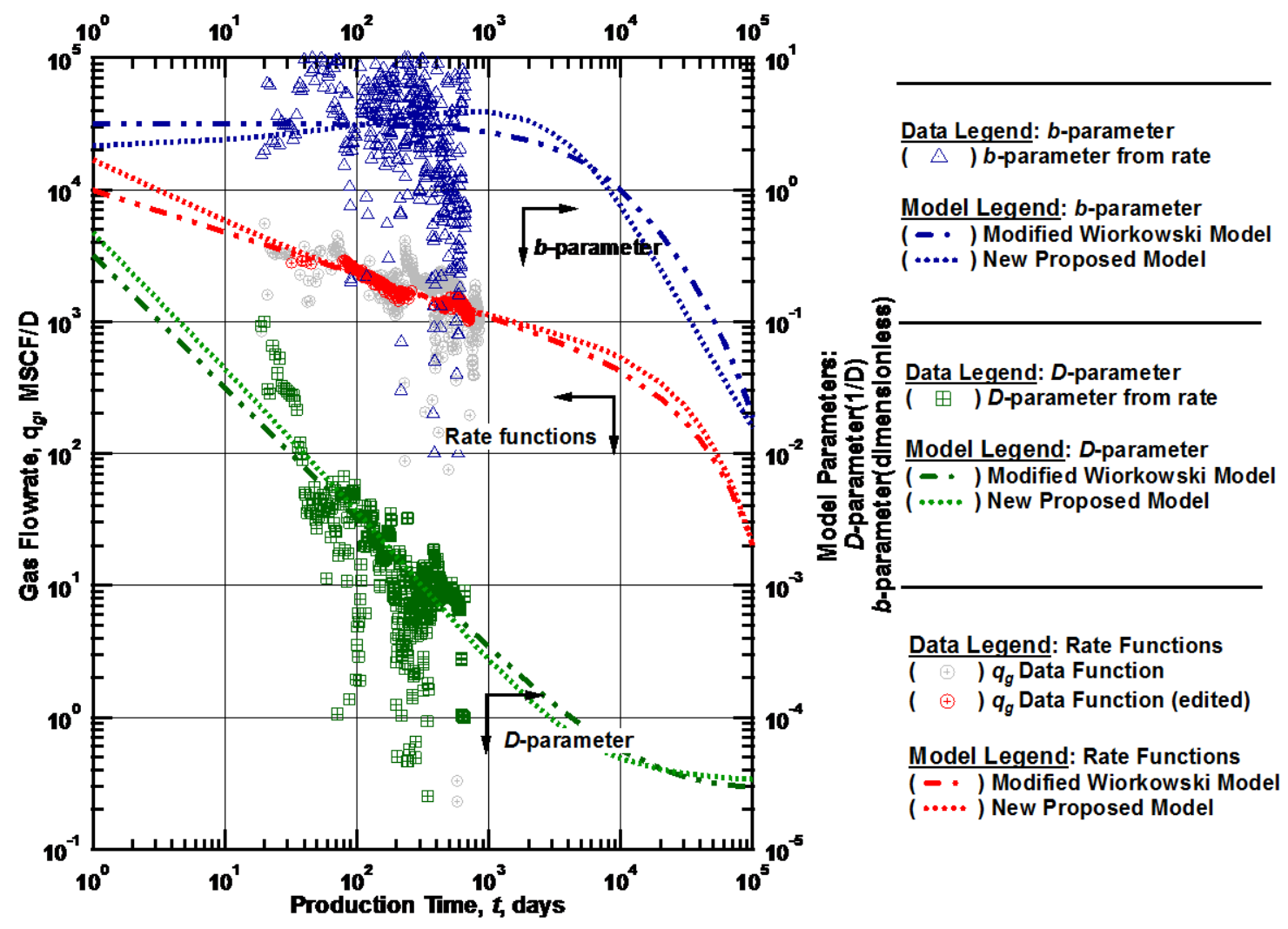

Figure E-46 - (Log-log Plot): $q D b$ plot - gas flow rate $\left(q_{g i}\right), D$ - and $b$-parameters versus production time and Modified Wiorkowski Model and Modified Ilk Model matches for Well 46. 
Modified Wiorkowksi and Proposed New Model qDb plot - Well 47

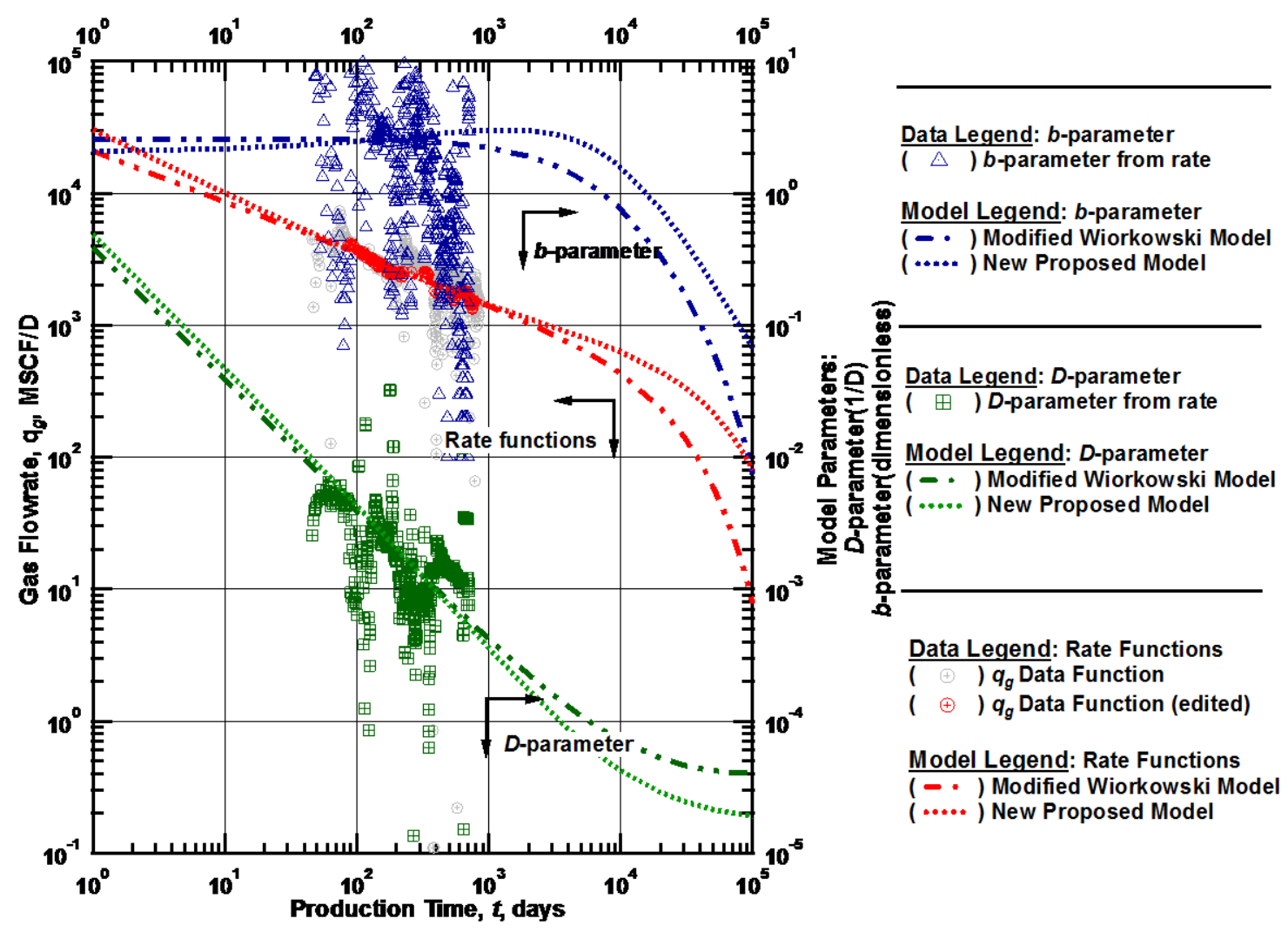

Figure E-47 - (Log-log Plot): $q D b$ plot — gas flow rate $\left(q_{g i}\right), D$ - and $b$-parameters versus production time and Modified Wiorkowski Model and Modified Ilk Model matches for Well 47. 
Modified Wiorkowksi and Proposed New Model qDb plot - Well 48

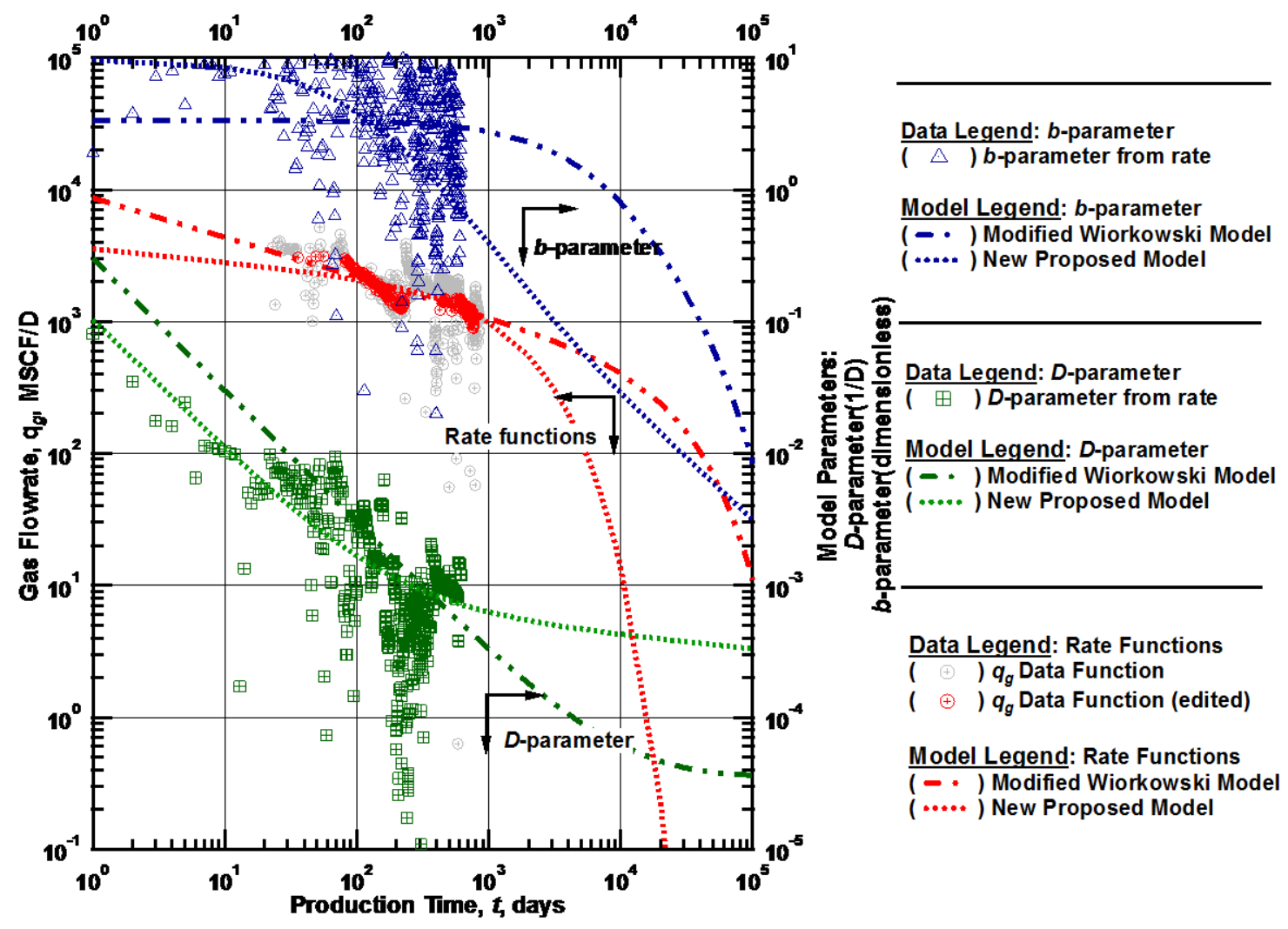

Figure E-48 - (Log-log Plot): $q D b$ plot - gas flow rate $\left(q_{g i}\right), D$ - and $b$-parameters versus production time and Modified Wiorkowski Model and Modified Ilk Model matches for Well 48. 
Modified Wiorkowksi and Proposed New Model qDb plot - Well 49

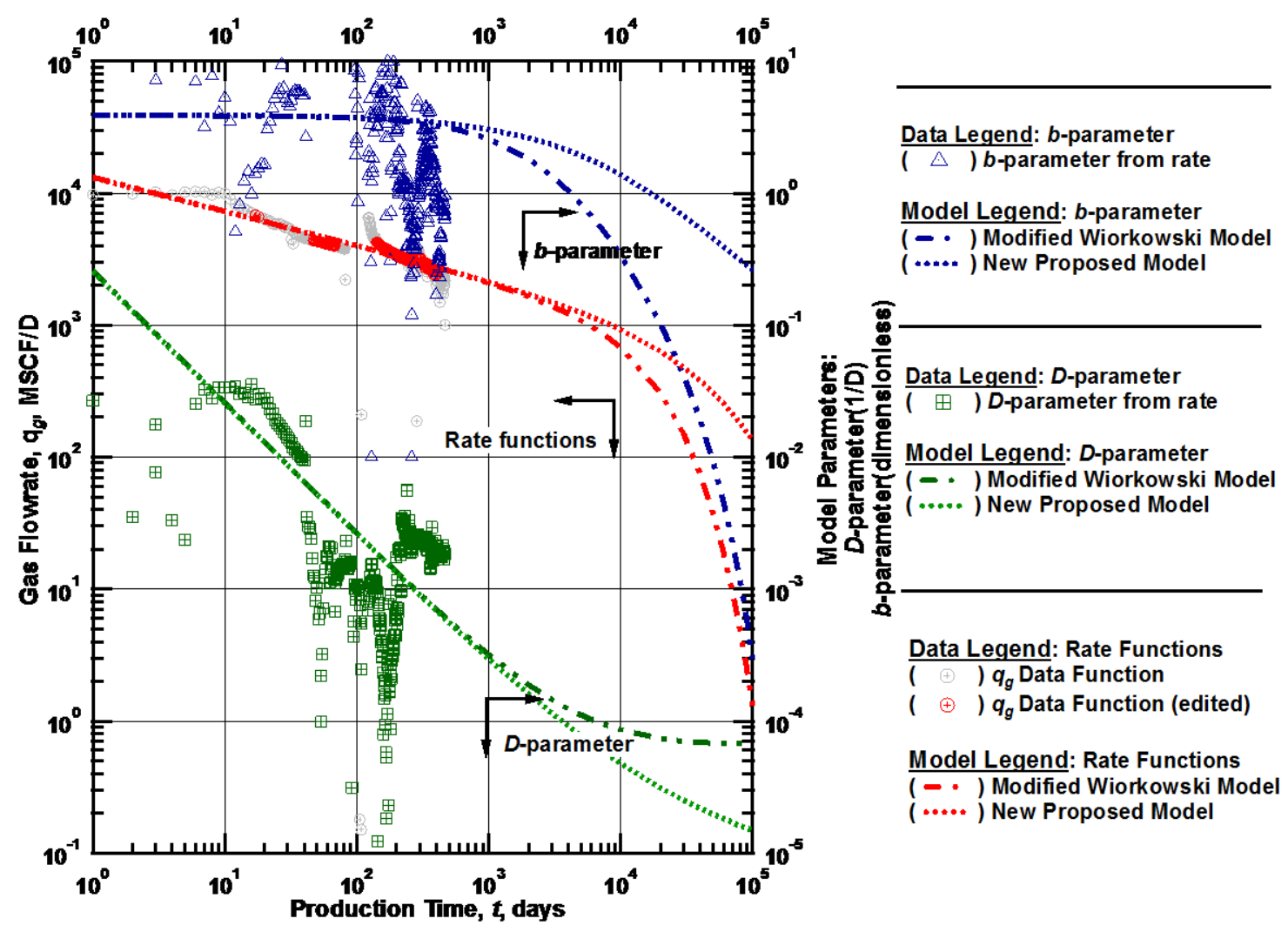

Figure E-49 - (Log-log Plot): $q D b$ plot - gas flow rate $\left(q_{g i}\right), D$ - and $b$-parameters versus production time and Modified Wiorkowski Model and Modified Ilk Model matches for Well 49. 
Modified Wiorkowksi and Proposed New Model qDb plot - Well 50

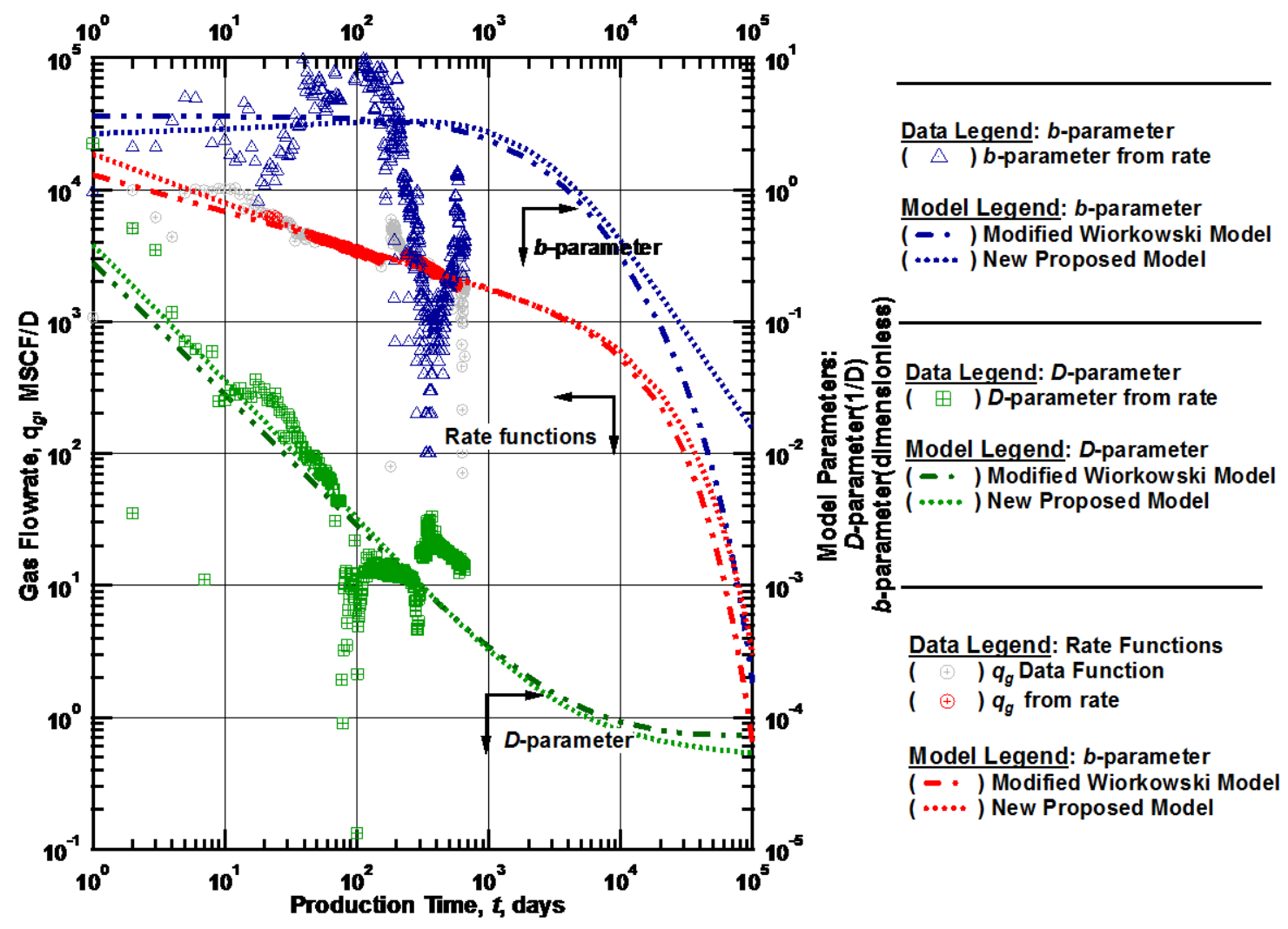

Figure E-50 - (Log-log Plot): $q D b$ plot — gas flow rate $\left(q_{g i}\right), D$ - and $b$-parameters versus production time and Modified Wiorkowski Model and Modified Ilk Model matches for Well 50. 
Modified Wiorkowksi and Proposed New Model qDb plot - Well 51

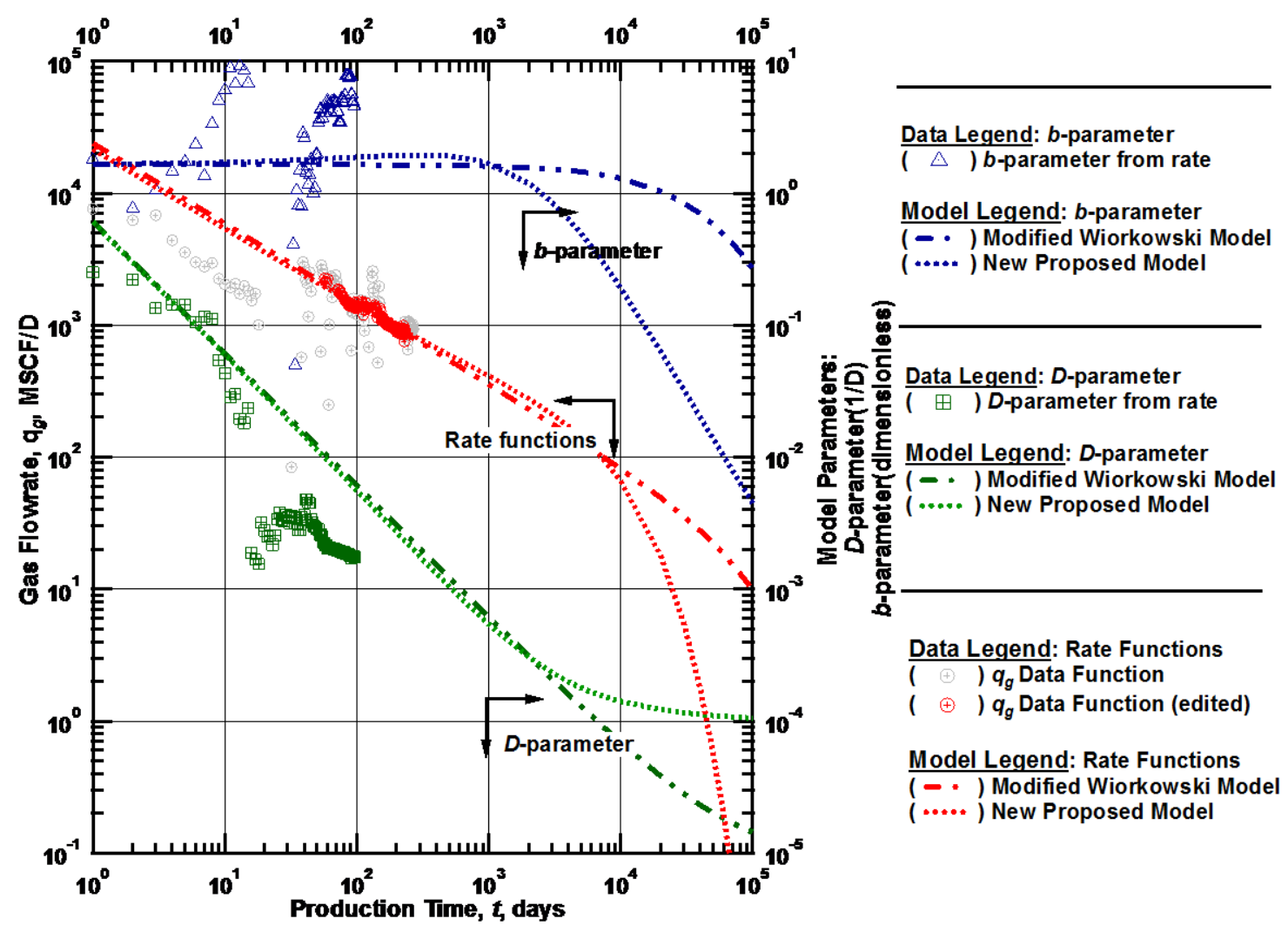

Figure E-51 - (Log-log Plot): $q D b$ plot — gas flow rate $\left(q_{g i}\right), D$ - and $b$-parameters versus production time and Modified Wiorkowski Model and Modified Ilk Model matches for Well 51. 
Modified Wiorkowksi and Proposed New Model qDb plot - Well 52

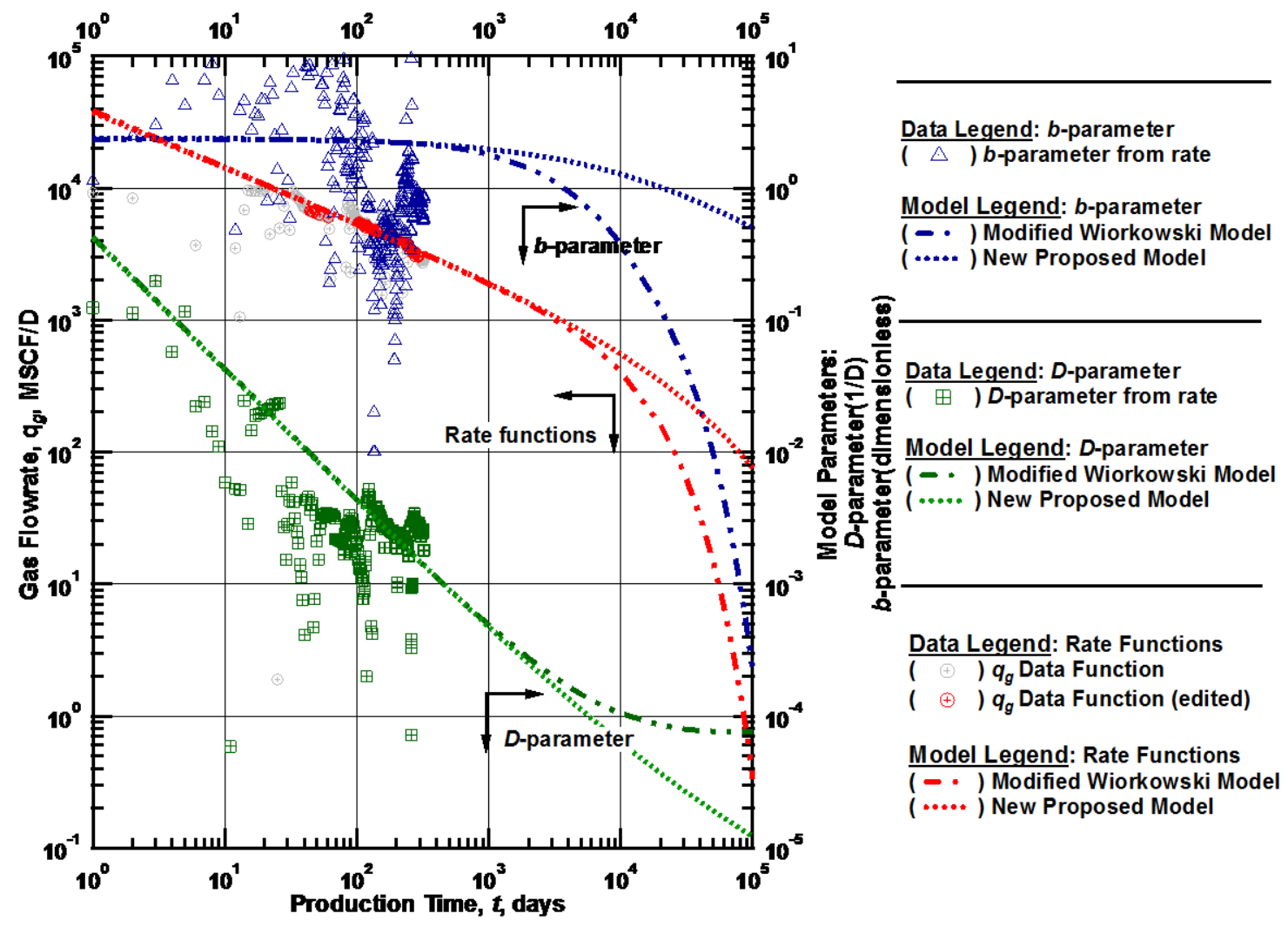

Figure E-52 - (Log-log Plot): $q D b$ plot — gas flow rate $\left(q_{g i}\right), D$ - and $b$-parameters versus production time and Modified Wiorkowski Model and Modified Ilk Model matches for Well 52. 
Modified Wiorkowksi and Proposed New Model qDb plot - Well 53

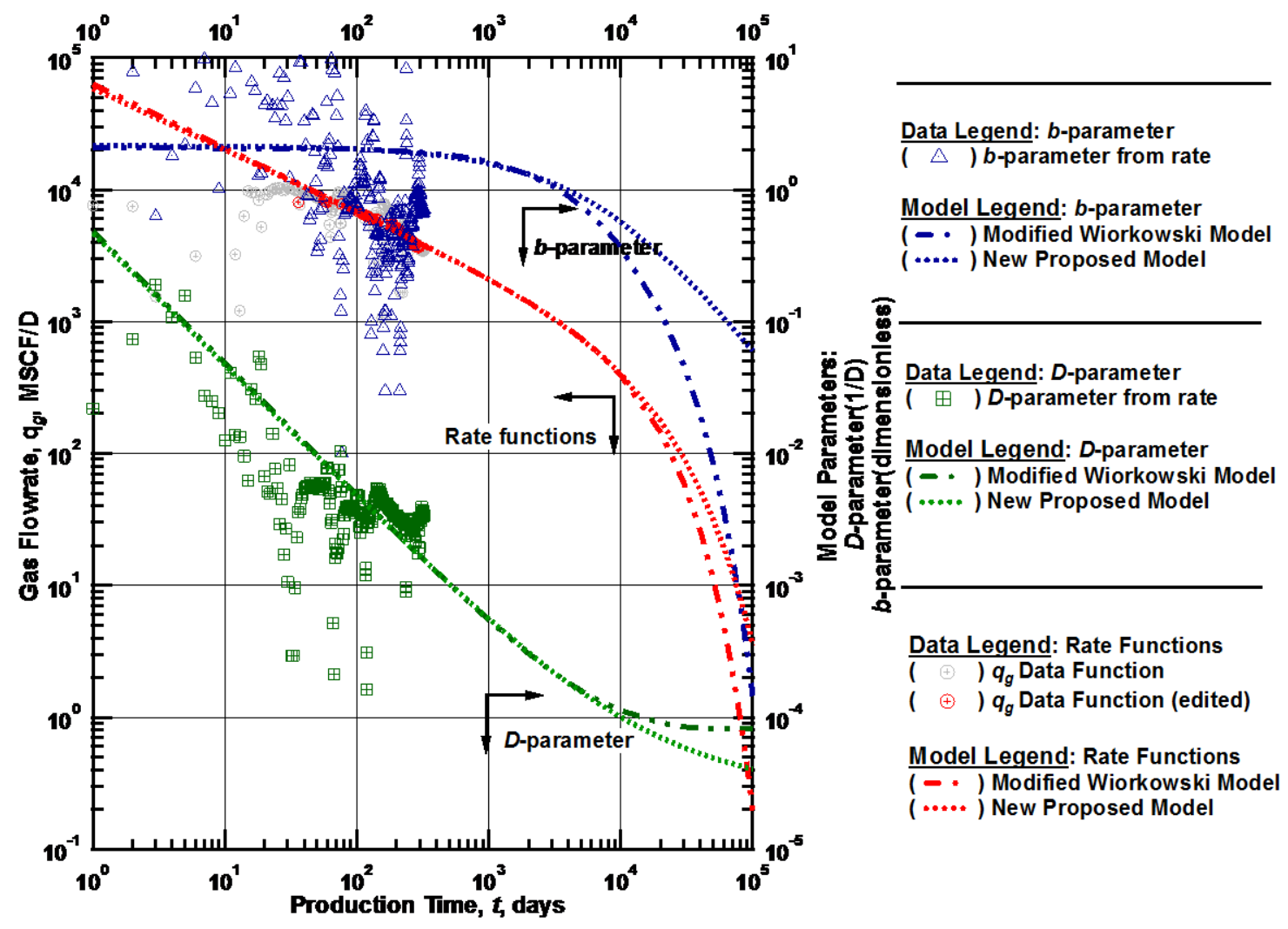

Figure E-53 - (Log-log Plot): $q D b$ plot — gas flow rate $\left(q_{g i}\right), D$ - and $b$-parameters versus production time and Modified Wiorkowski Model and Modified Ilk Model matches for Well 53. 
Modified Wiorkowksi and Proposed New Model qDb plot - Well 54

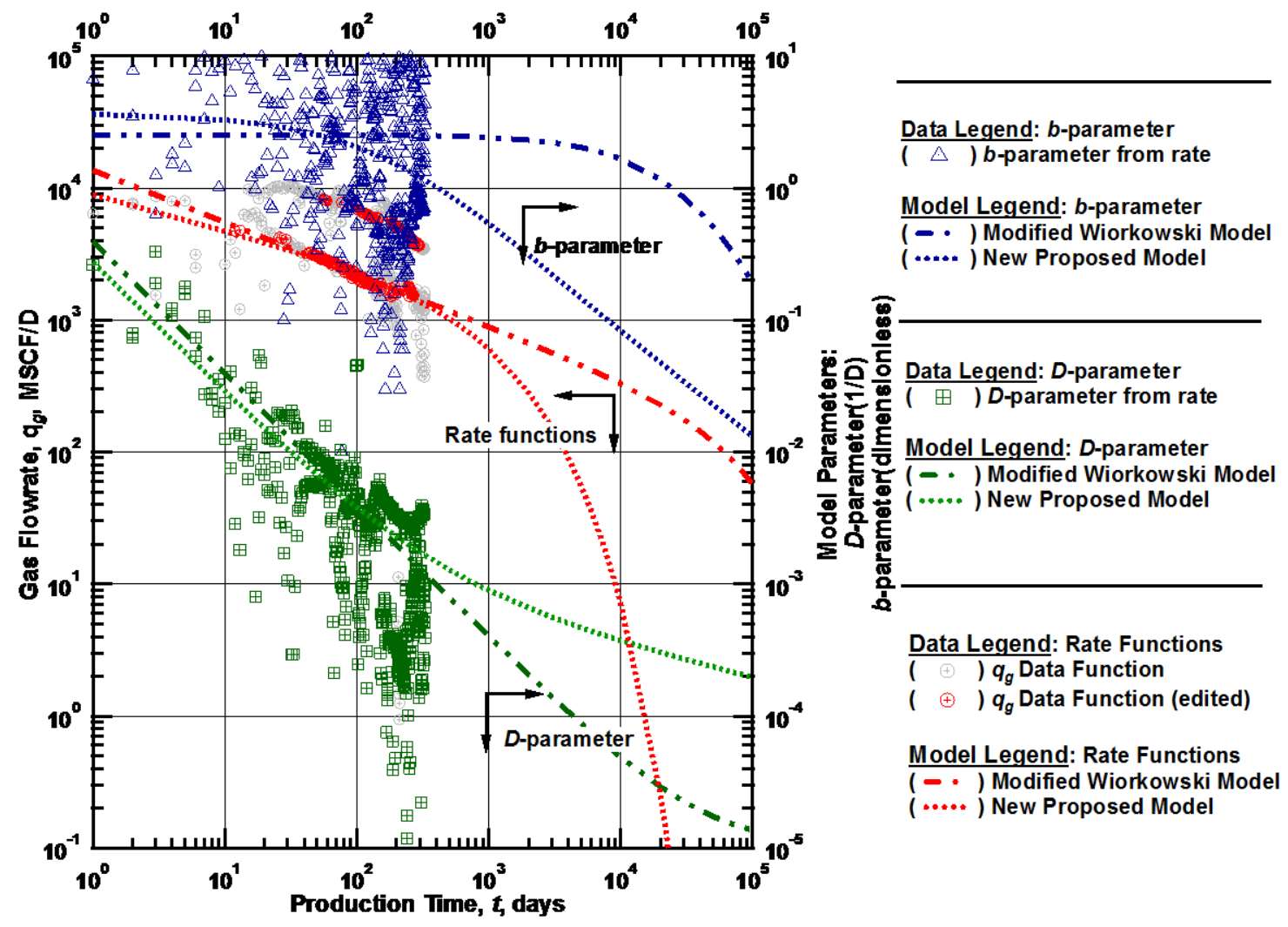

Figure E-54 - (Log-log Plot): $q D b$ plot — gas flow rate $\left(q_{g i}\right), D$ - and $b$-parameters versus production time and Modified Wiorkowski Model and Modified Ilk Model matches for Well 54. 
Modified Wiorkowksi and Proposed New Model qDb plot - Well 55

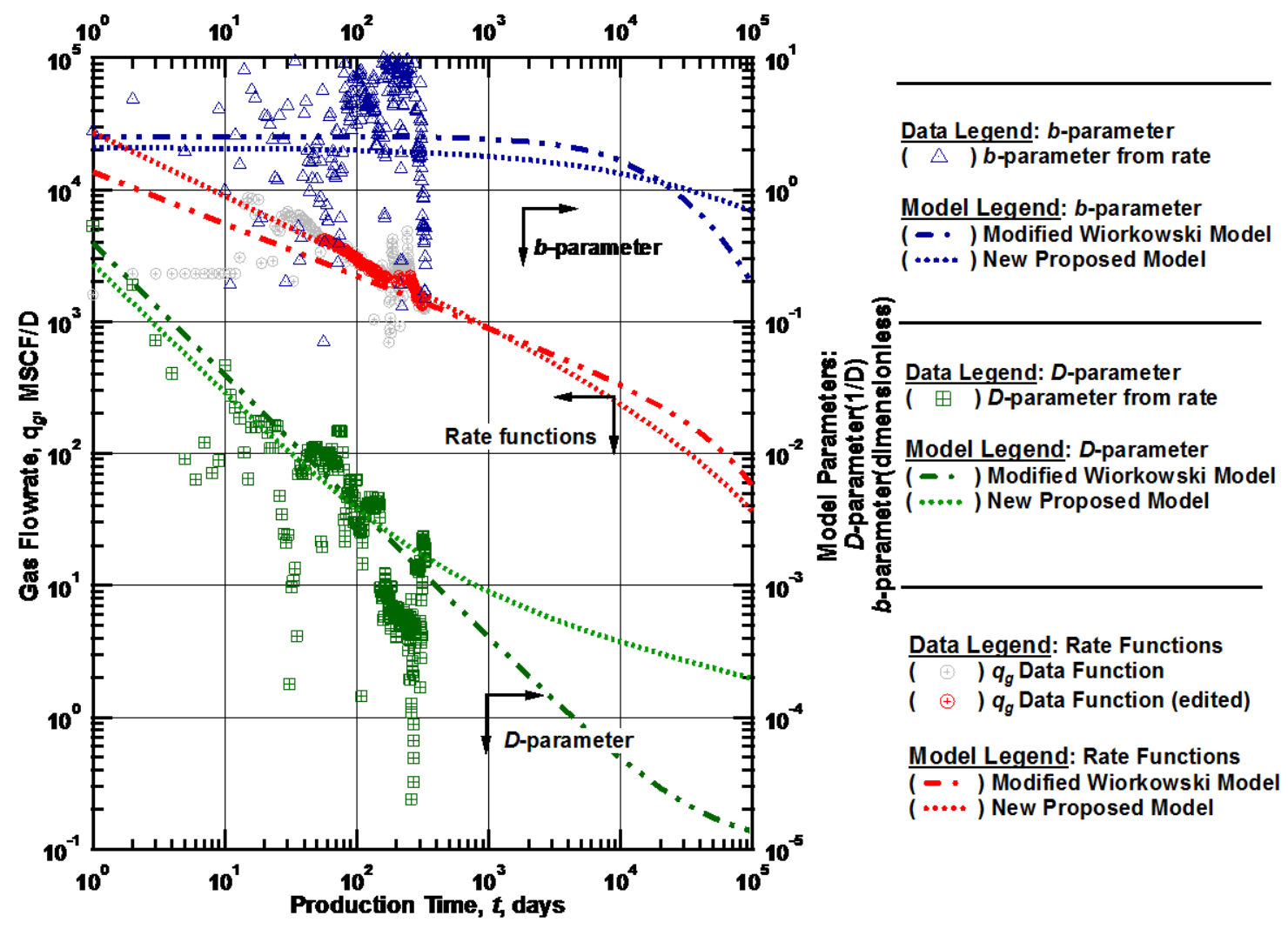

Figure E-55 - (Log-log Plot): $q D b$ plot — gas flow rate $\left(q_{g i}\right), D$ - and $b$-parameters versus production time and Modified Wiorkowski Model and Modified Ilk Model matches for Well 55. 